\title{
Entwicklung interkultureller Kompetenz in Deutsch als Fremdsprachenunterricht
}

\author{
Dissertation \\ zur Erlangung des sozialwissenschaftlichen Doktorgrades der \\ Sozialwissenschaftlichen Fakultät der Georg-August-Universität Göttingen
}

vorgelegt von

Oxana Fuchs

aus Irkutsk

Göttingen 2012 
1. Gutachterin Prof. Dr. Christina Krause

2. Gutachter Prof. Dr. Hatmut Schröder

Tag der mündlichen Prüfung 10.10.1011 


\section{Vorwort}

Das Thema „Interkulturalität“ begleitet mich seit meiner Studienzeit. Als ich noch Germanistik an der Linguistischen Universität in meiner Heimatstadt Irkutsk studiert habe, wurde ich zum ersten Mal mit den nationalen Stereotypen konfrontiert. Einige der dort unterrichtenden Dozenten waren der Meinung, dass die Studenten unterschiedlicher Fakultäten bestimmte Eigenschaften besitzen, so dass z.B. die Studenten der Fakultät für die deutsche Sprache besonders pünktlich, ordentlich und obrigkeitshörig sein sollten, deshalb versäumten sie weniger als Studenten der anderen Fakultäten die Vorlesungen und gaben meist rechtzeitig ihre Hausarbeiten ab. Studenten der Fakultät für die englische Sprache wären freiheitsliebend und kreativ, was in dem lässigen Umgang mit den Studienregeln und großer Selbständigkeit zum Ausdruck gebracht würde. Diese Eigenschaften wurden im Zusammenhang mit der zu erlernenden Sprache bzw. Kultur gebracht. Die Deutschen seien pünktlich, weshalb diejenigen, die diese Sprache für ihren Beruf lernten, auch pünktlich sein sollten. Ich habe mich damals gefragt, wie viel Wahres solche Aussagen wohl enthalten könnten. Die weiteren Recherchen in der wissenschaftlichen Literatur leiteten mein Interesse zu dem Thema „nationale Stereotypen“, und dann zum Thema „Interkulturalität“. Die Studienreisen nach Deutschland ermöglichten mir das Eintauchen in reale Situationen des interkulturellen Kontakts mit den Vertretern anderer Kulturen. Nicht selten geriet ich in peinliche Situationen aufgrund meines Unwissens über die kulturellen Eigenheiten meines Gesprächspartners oder fehlender Selbstreflektion, bzw. beobachtete die fehlgeschlagene Kommunikation. Ausschlaggebend für die Wahl des Dissertationsthemas war der Besuch des Seminars „Interkulturelle Kommunikation“ im Rahmen der Sommerkurse für die ausländischen Studierenden an der Universität Essen.

Meine Dissertation habe ich in Russland begonnen zu schreiben. Das durch das DAAD-Stipendium ermöglichte Forschungsjahr habe ich an der Universität Göttingen verbracht, wo ich meine wissenschaftliche Betreuerin Prof. Dr. Christina Krause kennen gelernt habe. An dieser Stelle möchte ich mich bei ihr für die freundliche Unterstützung dieser Arbeit, ihre Hilfe bei der fachlichen Auseinandersetzung mit dem Thema und ihre Herzlichkeit bedanken. Die Arbeit an der Dissertation nahm einige Jahre in Anspruch, die auch privat für mich schwer waren. Immer habe ich die Hilfsbereitschaft von Prof. Dr. Krause erlebt. Während des Forschungsjahres habe ich mich entschlossen, meine Arbeit in der deutschen Sprache zu verfassen und an der Universität Göttingen zu promovieren. Dieses Jahr ermöglichte auch die Zusammenarbeit mit dem inzwischen verstorbenen Prof. Dr. Kar- 
1-Heinz Flechsig, dem ich zahlreiche konstruktive Anregungen zu meiner Arbeit zu verdanken habe. Des Weiteren begleitete mich Dr. Erika Voigt in allen Höhen und Tiefen. Bei ihr bedanke ich mich dafür, dass sie mich motiviert und in jeder Hinsicht unterstützt hat, was maßgeblich zur Realisierung dieser Arbeit beigetragen hat.

Die Multikulturalität der deutschen Gesellschaft durfte ich bei der Arbeit in den Integrationskursen als Sprachdozentin erleben, was meine interkulturelle Kompetenz auf eine harte Prüfung stellte und weitere Überlegungen und praktische Beobachtungen für meine Dissertation lieferte.

Interkulturalität in allen Fassetten erlebe ich jeden Tag: als eine Ausländerin in der deutschen Gesellschaft, als eine Frau, die in einer multikulturellen Ehe lebt. Meinem Mann Armin Fuchs danke ich für alle themenbezogenen Diskussionen und auch für seine Geduld und Unterstützung. Mein Dank gilt letztlich auch meinen Eltern: meinem verstorbenen Vater Nikolai Chekalin und meiner Mutter Galina Chekalina.

In der multikulturellen Gesellschaft zu leben und sich darin interkulturell kompetent zu verhalten - das verstehe ich als Herausforderung, aber auch als Chance. Dies möchte ich meinem Sohn Maximilian Fuchs ermöglichen und ihn dabei unterstützen. 


\section{Inhaltsverzeichnis}

1 Aktualität der Arbeit und ihre Zielsetzung...............................................................

1.1 Die interkulturelle Kompetenz und die moderne Gesellschaft.................................6

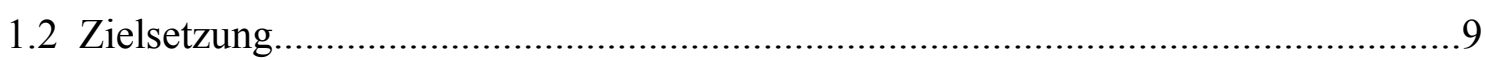

2 Die interkulturelle Kompetenz als neues Ziel des Fremdsprachenunterrichts.................12

2.1 Die Entwicklung der Zielvorstellungen für den Fremdsprachenunterricht - ein

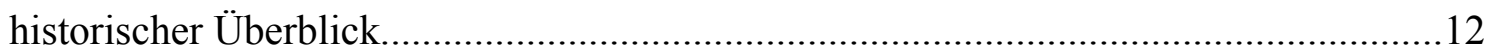

2.2 Zur aktuellen Diskussion von Begriffen aus der modernen Fremdsprachendidaktik

3 Theoretische Konzepte zur interkulturellen Kompetenz...... .34

4 Das Puzzle-Modell - ein Vorschlag für die Herausbildung der interkulturellen Kompetenz im Fremdsprachenunterricht.

4.1 Kulturspezifische und kulturübergreifende Kenntnisse als kognitive Komponente

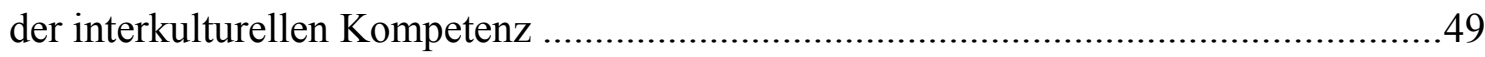

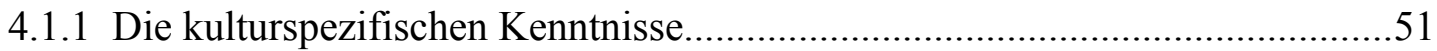

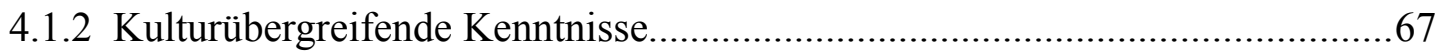

4.2 Die interkulturelle Sensibilität als affektive Komponente......................................77

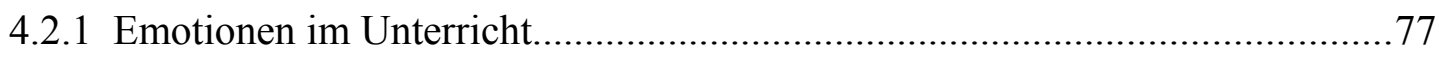

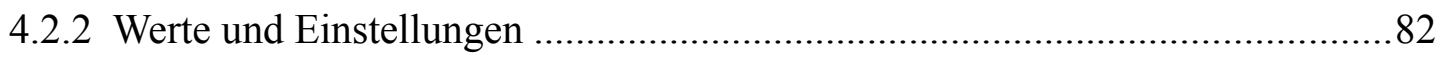

4.2.3 Entwicklungsmodelle zur interkulturellen Sensibilität.................................86

4.3 Die interkulturellen Fähigkeiten als pragmatische Komponente ...........................90

5 Programm zur Entwicklung der interkulturellen Kompetenz..................................... 102

5. 1 Einführung in das Programm: Ziel und Aufgaben.................................................102

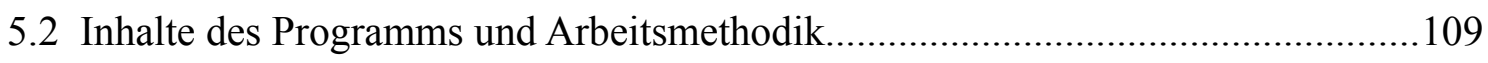

5.2.1 Fallbeispiele:Arbeitsmethodik und Beispiele aus dem Programm.................112

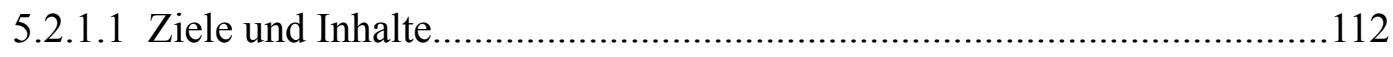

5.2.1.2 Didaktisch-methodische Umsetzung................................................113

5.2.2 Kulturelle Selbstreflexion: Arbeitsmethodik und Beispiele aus dem Programm

5.2.2.1 Ziele und Inhalte 
5.2.3 Vorgefasste Meinungen und Stereotype. Arbeitsmethodik und Beispiele aus dem Programm.

5.2.3.1 Ziele und Inhalte.

5.2.3.2 Didaktisch-methodische Umsetzung.

5.2.4 Landeskunde und Sprachübungen: Arbeitsmethodik und Beispiele aus dem

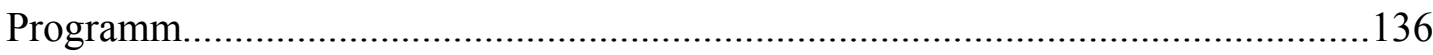

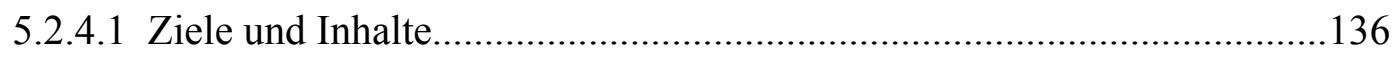

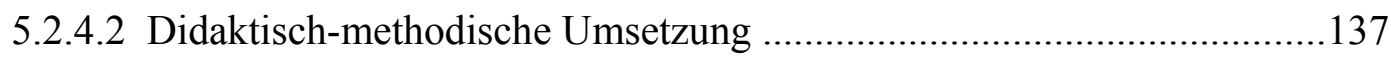

6 Das Experiment zur Umsetzung des Puzzle-Modells im Fremdsprachenunterricht......145

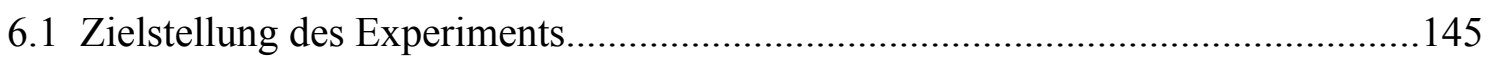

6.2 Experimentelle Bedingungen: Fremdsprachenunterricht in einer berufsorientierten

Hochschule (am Beispiel einer beruflichen Militäreinrichtung in Russland)

6.2.1 Professionelle Kompetenz als übergeordnetes Ziel der berufsbezogenen

Ausbildung.Die Bedeutung des interkulturellen Lernens für den weiteren beruflichen

Werdegang der Auszubildenden...........................................................................149

6.2.2. Ziele und Rahmen des Fremdsprachenunterrichts....................................... 152

6.2.3 Unterrichtsmaterial und Lehrwerk........................................................... 155

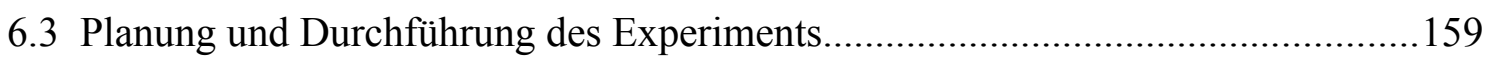

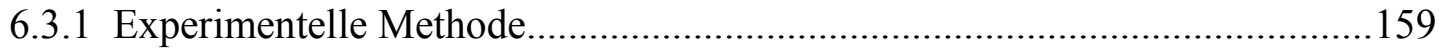

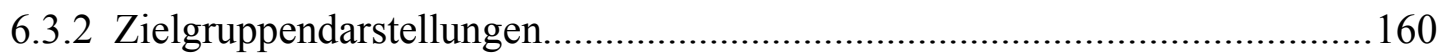

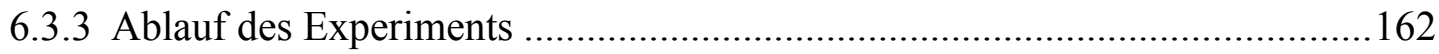

6.4 Auswertung der Ergebnisse des Experiments...................................................... 163

6.4.1 Instrument zur Evaluierung der Ergebnisse..............................................163

6.4.2 Datenbearbeitung und statistische Signifikanz..........................................169

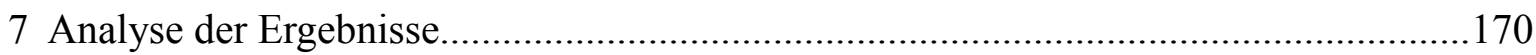

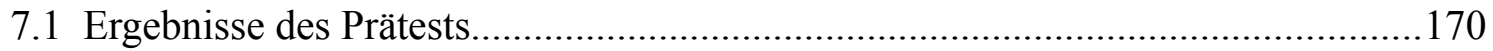

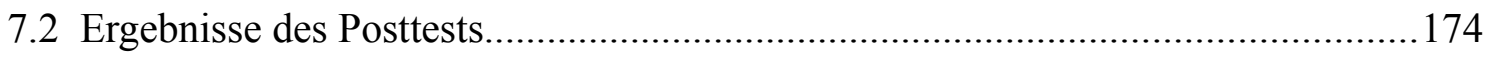

7.3 Veränderungen der interkulturellen Kompetenz entsprechend der drei Komponenten

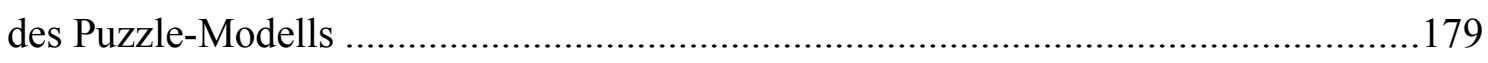

7.3.1 Veränderungen der Komponente „Kenntnisse“ “.......................................... 179

7.3.2 Veränderungen in der Komponente ,,interkulturelle Sensibilität““.................183 
7.3.3 Veränderungen in der Komponente „,interkulturelle Fähigkeiten“ 187

7.4 Prä- und Posttest-Vergleich - Gesamtergebnis......................................................191

7.4.1 Gesamtergebnis: interkulturelle Kompetenz - Zusammenfassung.................191

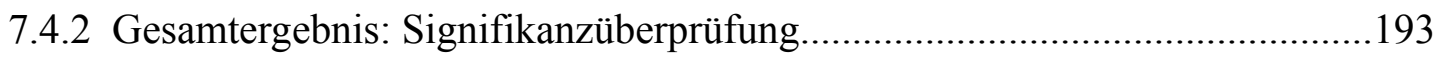

7.4.3 Vergleich der Veränderungen in den Gruppen mit unterschiedlichen

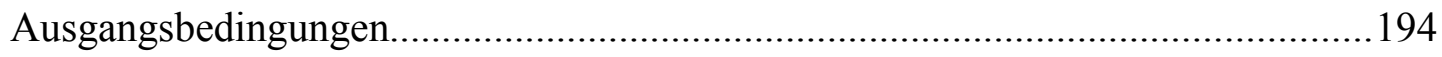

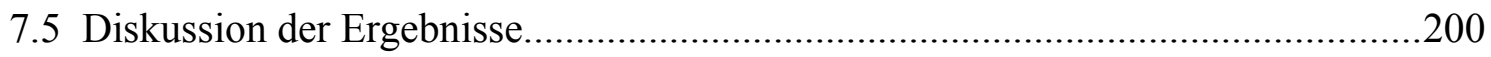

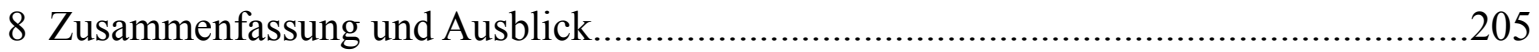

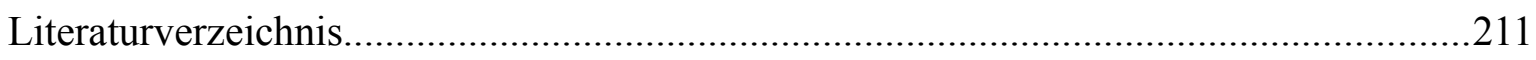

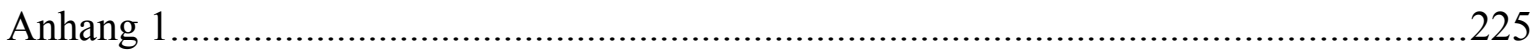

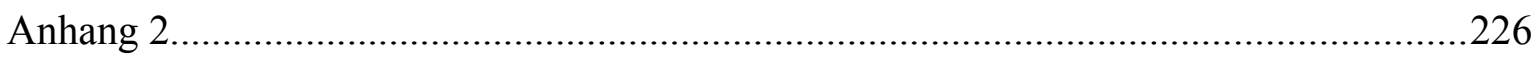




\section{Aktualität der Arbeit und ihre Zielsetzung}

\subsection{Die interkulturelle Kompetenz und die moderne Gesellschaft}

Im 21. Jahrhundert erleben viele Gesellschaften sowohl in Europa als auch in anderen Teilen der Welt rasante wirtschaftliche, politische, soziale und kulturelle Veränderungen. Die Verbindungen zwischen unterschiedlichen Ländern, Nationen und kulturellen Gruppen gewinnen an Intensität. Für die einzelne kulturelle Gemeinschaft, aber auch für jede Person entsteht daraus die folgende Problematik: einerseits die eigene kulturelle Identität zu wahren, andererseits sich an neue kulturelle Kontexte anzupassen. Um beiden Seiten gerecht zu werden, ist es sowohl für das einzelne Individuum als auch für die einzelnen kulturellen, ethnischen oder sozialen Gemeinschaften notwendig, interkulturelle Kompetenz zu entwickeln.

„Interkulturelle Kompetenz ist die Fähigkeit, effektiv und angemessen in interkulturellen Situationen zu kommunizieren, auf Grundlage eigenen kulturellen Wissens, Fähigkeiten und Einstellungen“ (Deardorff o.J., S. 14).

Die Kultur ist eine soziale Erscheinung, die alle Bereiche der Gesellschaft betrifft und das besondere Verhältnis zu deren Werten und Einstellungen charakterisiert.

„Kultur ist ein universelles, für eine Gesellschaft, Organisation und Gruppe aber sehr typisches Orientierungssystem. Dieses Orientierungssystem wird aus spezifischen Symbolen gebildet und in der jeweiligen Gesellschaft usw. tradiert. Es beeinflusst das Wahrnehmen, Denken, Werten und Handeln aller ihrer Mitglieder und definiert somit deren Zugehörigkeit zur Gesellschaft. Kultur als Orientierungssystem strukturiert ein für die sich der Gesellschaft zugehörig fühlenden Individuen spezifisches Handlungsfeld und schafft damit die Voraussetzungen zur Entwicklung eigenständiger Formen der Umweltbewältigung“. (Thomas 1993, S.380)

Im Rahmen des Konzepts ,interkulturelle Kompetenz“ wird Kultur auf keinen Fall wie eine konstante Erscheinung betrachtet, sondern als ein in Entwicklung befindlicher Prozess. Die Kultur wird von den Mitgliedern einer Gruppe als gemeinsame Basis geteilt. Sie bestimmt deren Weltansichten und Handlungen.

Während früher der Begriff „Kultur“ mit dem Begriff „,nationale Kultur“ gleichgesetzt werden konnte, zeigt die moderne Entwicklung, dass keine monokulturellen Gesellschaften existieren. Die moderne Gesellschaft ist vielfältig. Innerhalb eines Landes oder einer nationalen Kultur coexistieren mehrere Subkulturen, die Unterschiede in den Einstellungen, 
Werten, Sprachen, Verhaltensregeln etc. aufweisen. Das führt dazu, dass die Grenzen zwischen den Kulturen aufweichen, die Grenzen zwischen „eigen“ und „fremd“ verschwimmen und neue kulturelle Gemeinschaften entstehen.

Die Gründe für diese Erscheinungen sind vielfältiger Natur. Die interkulturelle Kommunikation entwickelt sich in unterschiedlichen Sphären des Lebens: im Tourismus, im Sport, in den international tätigen Konzernen und auf dem Niveau der privaten Kontakte. Aber auch Veränderungen des politischen und sozialen Lebens der einzelnen Staaten haben zu intensiver Migration geführt, d.h. zu Umsiedlung, Vermischung und Kollision der Völker. ,Multikulturelle Gesellschaft‘ ist ein Markenzeichen unserer Zeit. Dazu gehört die Ankunft von Migranten und Flüchtlingen, wie es in Deutschland der Fall ist, oder geschichtlich bedingte Vermischung unterschiedlicher nationaler Kulturen auf einem Territorium, wie in Russland bzw. in Sibirien. Die Gründe für ein Zusammenleben der unterschiedlichen Kulturen können verschiedene Ursprünge haben. Was bleibt, sind die Probleme der Anerkennung der fremden Werte, Einstellungen und Verhaltensregeln und die Erkenntnis der eigenen kulturellen Prägung. Aus diesem Grund ist die Entwicklung der interkulturellen Kompetenz die Aufgabe der gesamten Gesellschaft und muss ein Teil der Sozialpolitik werden. Dabei ist folgende Einstellung wichtig: Jeder Mensch muss mit Würde und Respekt behandelt werden, unabhängig von seinem kulturellen Ursprung. Die Kulturen sind in ihrer Natur offene Konstrukte, sie können sich unter dem Zusammenwirken einzelner Menschen und sozialer Gruppen verändern.

Die Entwicklung der interkulturellen Kompetenz ist kein selbstlaufender Prozess. Das Vorhandensein der interkulturellen Beziehungen im Leben eines Menschen, z.B. zu den Nachbarn oder Kollegen, die aus einer anderen Kultur stammen, macht diesen Menschen nicht automatisch interkulturell kompetent. Diese Entwicklung erfordert sowohl langfristige Arbeit und Bemühungen jedes einzelnen Menschen als auch Vertrauen und Hingabe. Es wird von jedem Teilnehmer der interkulturellen Begegnung die Bereitschaft gefordert, die eigene kulturelle Einstellung zu reflektieren. Der Erwerb der interkulturellen Kompetenz ist eine Chance, den Ethnozentrismus, durch dessen Prisma andere Kulturen wahrgenommen werden, zu bewältigen. Die kulturelle Vielfalt eröffnet neue Möglichkeiten, die eigene Weltsicht zu vervielfältigen und zu bereichern.

Bei der Lösung der heutigen Probleme sowohl der deutschen als auch der russischen Gesellschaft wird die interkulturelle Kompetenz angefordert. Indem Russland eine Phase der Integration in die europäische und weltweite Wirtschaft erlebt, zerreißen die internationalen Konflikte das Innere des Landes. Während diese Konflikte vor einigen Jahren meistens 
auf dem Territorium der ehemaligen Sowjetrepubliken wie z.B. Tschetscheno-Inguschetische Republik ausgetragen wurden, werden sie heute immer häufiger auf dem Gebiet der russischen Föderation initiiert. Russland erlebt zurzeit einen der heftigsten Arbeitsmigrantenzuflüsse in seiner Geschichte, was einen Zuwachs der russischen nationalistischen Bewegungen zur Folge hat. Dabei werden die interkulturellen Konflikte nicht nur auf Diskussionsbasis gelöst, vielmehr kommt es häufig zu brutalen Auseinandersetzungen als Lösungsstrategien für solche Konflikte. Im Unterschied zu einigen europäischen Ländern ist der Mangel an interkultureller Kompetenz, die bei solchen Problemen dringend benötigt wird, kein Thema, das in der russische Politik aufgenommen und diskutiert wird.

Die Lage in Deutschland ist zwar anders, aber auch sie ist durch viele Probleme gekennzeichnet. Das Positive dabei ist, dass das Thema der interkulturellen Kompetenz zu einem wichtigen Punkt der politischen Debatten geworden ist. Die stetig wachsende Zahl der Migranten und ihre schlechte Eingliederung in die deutsche Gesellschaft zwingen die Politiker, die misslungene Integration zu verbalisieren. Probleme, wie zum Beispiel schlechte Kenntnisse der deutschen Sprache oder Bildung von Gettos, in denen die nationalen Traditionen über den Rechten eines einzelnen Menschen stehen, erfordern dringend Maßnahmen seitens der Politik. So wurde z.B. die Kontrolle über den Besuch von Integrationskursen verschärft und das Gesetz über die strafrechtliche Verfolgung im Fall einer Zwangsheirat verabschiedet. Das sind aber nur Teillösungen. Von den Politikern wird genauso wie von den Menschen mit Migrationshintergrund interkulturelle Kompetenz gefordert. Der Fall des ehemaligen Finanzsenators und SPD-Mitglieds Thilo Sarrazin, der öffentlich diskriminierende Äußerungen über arabische und türkische Einwanderer gemacht hat, zeigt, dass auch auf politischer Ebene Bedarf an interkultureller Aufklärung besteht. Die Äußerungen von Sarrazin lösten eine breite öffentliche Diskussion aus, viele Einheimische fühlten sich in ihren ausländerfeindlichen Gedanken bestätigt. Mangel an interkultureller Sensibilität kommt in solchen Situationen, wo nur die Bedeutung des Fremden übergewichtet wird und das Gemeinsame aus den Augen verloren wird, zum Ausdruck und kann zur Spaltung der Gesellschaft und später zu internationalen Konflikten führen. Umso wichtiger ist die Idee der interkulturellen Aus- und Weiterbildung, die für jedes Mitglied einer modernen Gesellschaft, mit oder ohne Migrationshintergrund, aktuell ist.

Die interkulturelle Kompetenz gehört zu den Schlüsselkompetenzen unserer Zeit. Sie ist ein Kompass, mit dem jeder Einzelne sich durch die Welt der kulturellen Vielfalt lotsen kann. 


\subsection{Zielsetzung}

In der wissenschaftlichen Literatur lässt sich eine Blütezeit für unterschiedliche Ausarbeitungen und Theorien über interkulturelle Kompetenz beobachten, wobei deren Bedeutung und Inhalt unterschiedlich diskutiert werden. Wissenschaftler/innen aus der Soziologie, Pädagogik, Didaktik und Psychologie entwickelten jeweils ihre eigenen Modelle der interkulturellen Kompetenz. Es entstanden in Verbindung mit dem Begriff „,interkulturell“ neue Zweige dieser Wissenschaften, so z.B. interkulturelle Pädagogik, interkulturelle Psychologie usw. Dabei haben die meisten Konzepte rein theoretischen Charakter und ziehen keine praktischen Ausarbeitungen nach sich.

Eine andere Welle des Interesses an der interkulturellen Kompetenz nimmt ihren Anfang in der Praxis des interkulturellen Managements. Das sind interkulturelle Trainings für die Mitarbeiter internationaler Unternehmen, die in ein anderes Land gesendet werden. Hier werden meistens praktische Ausarbeitungen und Programme entwickelt und von knappen Theorien begleitet.

Dabei ist die Verknüpfung von Theorie und Praxis so wichtig. Ein Konzept wird nur dann vollendet, wenn eine gut ausgearbeitete theoretische Basis durch eine praktische Realisierung ergänzt wird. Das ist ein Mangel, von dem das Konzept der interkulturellen Kompetenz bisher noch gekennzeichnet ist.

Interkulturelle Kompetenz wird zu einem immer bedeutenderen Anliegen und sollte auch Bildungsziel in Schulen und Hochschulen werden. Empfehlungen von Wissenschaftlern, interkulturelles Lernen in der alltäglichen Unterrichtsplanung zu berücksichtigen und sie zum Unterrichtsprinzip werden zu lassen, stoßen nicht immer auf das Verständnis seitens der Leitung und des Personals einer Lehreinrichtung. Die Trägheit des Ausbildungssystems trägt auch dazu bei, dass die neuen Konzepte und Ideen wenig Realisierung in der Praxis finden.

Das Problem betrifft alle Lehreinrichtungen, sei es Schule, Fachschule oder Universität, und tangiert auch alle Bildungsbereiche, insbesondere auch den Fremdsprachenunterricht. Interkulturelle Kommunikation ist schon lange ein wichtiges Thema für die Fremdsprachendidaktiker. Es wird jedoch oft auf theoretischem Niveau darüber diskutiert, die Realisierung in der Praxis steht noch aus. Während die Forderungen, die interkulturelle Kompetenz als neues Ziel des Fremdsprachenunterrichts zu erklären, immer lauter werden, fehlt es an neuen Methoden und an neuen Ideen.

Die vorliegende Arbeit soll ein Beitrag zur Lösung dieser Problematik sein, indem ein neu- 
es Modell der interkulturellen Kompetenz entwickelt und im Fremdsprachenunterricht erprobt wird.

In der Arbeit werden folgende Ziele verfolgt:

- Im ersten Kapitel wird die Aktualität des Themas besprochen und anschließend werden die Ziele der Arbeit definiert.

- Im zweiten Kapitel werden die literarischen Quellen aus den Bereichen Fremdsprachendidaktik,interkulturelle Pädagogik, Soziologie, interkulturelle Psychologie und Kulturwissenschaft bezüglich ihrer Aussagen zum Thema „interkulturelle Kompetenz" analysiert und im Zusammenhang mit den Zielen des Fremdsprachenunterrichts, mit den Begriffen der modernen Sprachdidaktik und mit den Konzepten der interkulturellen Kompetenz diskutiert.

- Im dritten Kapitel werden die Theorien von Fischer (2005), Fantini (2000) und Byram (1997) Brislin (1994) und Furmanowa (1993) diskutiert.

- Im vierten Kapitel wird ein von der Autorin entwickeltes Modell der interkulturellen Kompetenz präsentiert. Dieses „Puzzle-Modell“ zielt auf die drei Ebenen des Lernens: die kognitive, affektive und pragmatische. Dementsprechend besteht das Modell aus drei Komponenten: den kulturspezifischen und kulturübergreifenden Kenntnissen, der interkulturellen Sensibilität und den interkulturellen Fähigkeiten.

- Im fünften Kapitel wird das zur Umsetzung des Puzzle-Modells in der Praxis von der Verfasserin erarbeitete Programm zur Entwicklung der interkulturellen Kompetenz (PEIK) im Deutsch als Fremdsprache-Unterricht präsentiert. Dieses Programm, das im Folgenden PEIK genannt wird, wird als begleitende Maßnahme zum traditionellen Fremdsprachenunterricht verstanden. Es vereint das Gedankengut und die Methoden der interkulturellen Didaktik und interkulturellen Trainings in sich. Das Programm ist für Studenten bestimmt, die Deutsch als Fremdsprache in einer Lehreinrichtung in Russland während der Berufsausbildung erlernen, z.B. in einer Fach-, Hochschule oder in einer Universität. Das Ziel von PEIK besteht darin, durch und mit Hilfe einer Fremdsprache und mit interkulturell bedeutsamen Informationen und Handlungen die Lernenden auf Situationen des interkulturellen Kontakts vorzubereiten. Das Programm ist in 24 Themen aufgeteilt.

- PEIK, seine vier methodischen Blocks, deren Inhalte und die Arbeitsmethodik werden in der vorliegenden Arbeit präsentiert. Es handelt sich um:

1. Fallbeispiele, in denen interkulturelle Missverständnisse mit Methoden wie 
Kultur-Assimilator, offener Fall, Analyse des kritischen Ereignisses ${ }^{1}$ bearbeitet werden;

2. kulturelle Selbstreflexion, die durch verschiedene analytische Aufgaben und Spiele geübt werden kann.

3. vorgefasste Meinungen und Stereotype, zu deren Reflexion und Bewusstmachung verschiedene Aufgaben gestellt werden;

4. Landeskunde und Sprachübungen, die sich unmittelbar auf die Vermittlung der deutschen Sprache und der deutschen Kultur in Russland konzentrieren.

- Im sechsten Kapitel wird das Experiment zur Überprüfung der Durchführbarkeit des Programms vorgestellt. Es wurde in dem Ost-Sibirischen Institut des Ministerium des Inneren (Irkutsk, Russland) durchgeführt. An dem Experiment nahmen 30 Studierenden teil, es wurde im Laufe eines Semesters durchgeführt.

- Da das Konzept der interkulturellen Kompetenz bislang keine einheitliche Realisierung gefunden hat, weder in der Theorie noch in der Praxis, sind Probleme der Messung vorprogrammiert. Zur Zeit existiert kein Messinstrument, das von den Vertretern unterschiedlicher Richtungen gleichermaßen akzeptiert wird. Die auf dem Wissensmarkt vorhandenen Messinstrumente beziehen sich jeweils auf ein bestimmtes Modell und sind meistens auf die Evaluierung nur einer Seite der interkulturellen Kompetenz ausgerichtet. Die Autorin setzt sich mit diesem Problem auseinander und schlägt ein eigenes Messinstrument vor, das dem Puzzle-Modell angepasst ist und im Folgenden Puzzle-Fragebogen genannt wird. Der Puzzle-Fragebogen ist ein Selbstevaluierungsinstrument, das auf der Grundlage des Yoga-Tests von Fantini (2000) ausgearbeitet wurde.

- Im siebten Kapitel werden die Ergebnisse einer ersten Erprobung des von der Verfasserin erarbeiteten Programms PEIK vorgestellt. Zur Evaluierung wurde des ebenfalls von der Verfasserin entwickelte Instrument zur Messung des Entwicklungsniveaus der am Experiment teilnehmenden Studierenden eingesetzt. Dafür wurden Prä- und Posttest in den experimentellen Gruppen und zum Vergleich auch in Kontrollgruppen durchgeführt. Der Vergleich der Ergebnisse soll prüfen, ob PEIK im Unterrichtsfach „Deutsch als Fremdsprache“ einsetzbar ist bzw. welche Veränderungen für einen effektiven Einsatz noch notwendig sind.

- Im achten Kapitel werden die Inhalte der Arbeit zusammengefasst, ihre Grenzen aufgezeigt und ein Ausblick in die Entwicklungsmöglichkeiten der Arbeit gegeben.

Die Methoden werden später im Kapitel 5.2.1. definiert. 


\section{Die interkulturelle Kompetenz als neues Ziel des Fremdsprachenunterrichts}

\subsection{Die Entwicklung der Zielvorstellungen für den Fremdsprachenunterricht - ein historischer Überblick}

Im folgenden Kapitel wird ein historischer Überblick über die Entwicklung der Ziele des Fremdsprachenunterrichts gegeben. Da die meisten Zielvorstellungen übergreifend für alle im Unterricht erlernten Sprachen gelten, wird die Aufmerksamkeit nicht auf ein bestimmtes Fach fokussiert. Für die vorliegende Arbeit ist aber die Entwicklung der didaktischen Ziele auf dem Territorium der russischen Föderation wichtig, deshalb werden Theorien bevorzugt, die aus diesem Raum stammen. Zitate aus russisch-sprachigen Texten wurden von der Verfasserin übersetzt.

Das Studium der Fremdsprachen hat eine lange Geschichte. In dem Moment, als der Mensch die Grenzen seines Wohngebiets überschritt, begann er, Fremdsprachen zu lernen. Er war gezwungen, mit den Vertretern anderer Gesellschaften zu kommunizieren, und dafür brauchte er andere Sprachen. Die Fremdsprache wurde unmittelbar in der betreffenden Kultur erlernt und wurde nicht als Selbstzweck betrachtet, sondern als das Werkzeug, das für die Erkenntnis der umgebenden Welt, für den Erwerb der sozialen Erfahrung notwendig ist. Diese Herangehensweise wurde in der Literatur manchmal als realistischer Ansatz bezeichnet. „Ursprünglich verfolgte das Studium einer Fremdsprache pragmatische und kosmopolitische Ziele“ (Buttjes 1995, S. 143). Parallel mit der Sprache wurde auch das Wissen über die Welt und über das Land vermittelt, was bei der Orientierung im fremden Land half.

In späteren Jahren wurden Sprachen in das Lehrprogramm der Bildungseinrichtungen aufgenommen. Bis zum 18. Jahrhundert wurden aber nur antike oder sogenannte „tote“ Sprachen in den Bildungseinrichtungen vermittelt, und zwar größtenteils Latein sowie Griechisch. Die Beherrschung dieser Sprachen war die Grundlage für das Studium anderer Fächer, zum Beispiel Philosophie, Literatur, Religion oder Medizin. Die modernen Sprachen wurden in das Programm der Ausbildung nicht eingegliedert. Die modernen Fremdsprachen wurden entweder durch Auslandsaufenthalte oder auch durch private Kontakte erlernt. Es ist bekannt, dass viele adelige Kinder im 18. Jahrhundert von französischen Lehrern erzogen wurden, die oft keine richtige Lehrerausbildung hatten. Durch den Austausch mit den Erziehern lernten die Kinder eine Fremdsprache sozusagen „live“.

Der realistische Ansatz zum Erlernen von Fremdsprachen ist bis zum späten 19. Jahrhun- 
dert erhalten geblieben, als die Reformen der Ausbildung im europäischen Raum durchgeführt worden waren und die modernen Fremdsprachen in das Programm der Schulausbildung aufgenommen waren. Es existierten noch keine Unterrichtstraditionen oder Unterrichtsmethoden bezüglich der modernen Sprachen, die Didaktik des Unterrichtens der ,toten Sprachen“" wurde auf das Erlernen der modernen Fremdsprachen übertragen. Im Unterschied zu den „toten“ Sprachen, deren Gebrauch sich nur auf das Erlernen schriftlicher Texte in den anderen Fächer beschränkte, sollten die modernen Sprachen dem lebendigen Informationsaustausch und der Verständigung dienen.

Bei der Entwicklung der Ziele des Fremdsprachenunterrichts gab es unterschiedliche Ansätze, bei denen jeweils eine andere Kompetenz im Mittelpunkt stand.

\section{Grammatikalische Kompetenz}

Bei diesem Ansatz wurde die grammatische Seite der Sprache hervorgehoben. Jedes grammatikalische Thema wurde mittels Texten und Sätzen gelernt. Die Übersetzung war die üblichste Arbeitsform. Da die Schwerpunkte im grammatischen Bereich lagen, erhielt lexikalische Arbeit eine geringere Priorität. Andere Ziele, wie zum Beispiel der Gebrauch der auswendig gelernten grammatikalischen Strukturen in der Rede oder das Erlernen der kulturellen oder nationalen Besonderheiten des fremden Landes, fanden überhaupt keinen Niederschlag in der Praxis des Fremdsprachenunterrichts . „Wie ein Didaktiker witzig bemerkt hat, gelang es den Schülern, den Wortschatz der erlernten Sprache nur bei Pauken der grammatikalischen Ausnahmen kennen zu lernen“ (Werestschagin und Kostomarov 1983, S. 18). ${ }^{2}$ Die Grammatik wurde in einer Reihe von Übungen und Texten verankert, bei deren Auswahl kontextuelle Beziehungen und der Wert dieses Textes vom Gesichtspunkt der Kommunikation nicht berücksichtigt waren.

„Der Anspruch, alle potentiellen Realisierungen der sprachlichen Form zu präsentieren, rückte den Gebrauchswert der Strukturen für alltägliche Interaktionvollzüge ebenso in den Hintergrund wie die funktionale, d.h. pragmatische Verwendungsebene. Selbst wenn sprachliche Elemente als Frage-Antwort-Situationen präsentiert wurden, lasen sie sich mehr wie ein Verhör als ein Gespräch“"(Gehring 2002, S. 73). Der Sprachunterricht war einem Studio für Gehirn-Jogging ähnlich, wo Denkschulungen geübt wurden. Die praktische Bedeutung der Sprache für eine weitere Kommunikation war ausgeschlossen und wurde nicht als Ziel des Fremdsprachenunterrichts angesehen.

Der tschechische Pädagoge Johann Amos Comenius (1592-1670) hat durch seine didakti-

2 Alle russischsprachigen Zitaten sind von der Verfasserin übersetzt wurden. 
schen Arbeiten viel zur Ablehnung des grammatikalischen Verfahrens und zur Annäherung an die sprachliche Seite im Fremdsprachenunterricht beigetragen. Comenius behauptete, die Sprache müsse gleichzeitig mit der realen Umwelt erlernt werden. Er war der Meinung, dass die Sprachbeherrschung parallel zu der Beherrschung der nicht sprachlichen Wirklichkeit stattfinden soll. Er hat den Gedanken über die unzertrennliche Verbindung einer Sprache und einer Kultur entwickelt, denn die Kultur ist auch innerhalb einer künstlich geschaffenen Umgebung eines Klassenzimmers präsent. Die Vernachlässigung dieser These im Unterricht führte seines Erachtens zum Verbalismus. Unter Verbalismus wurde verstanden, dass die von dem Schüler ${ }^{3}$ verwendeten Wörter keine passende Bedeutung beinhalten, d.h. der Schüler kann zwar bestimmte Aussagen machen, welche aber für ihn keine entsprechende Bedeutung haben. Die Ideen von Comenius haben eine wichtige Rolle bei der Entwicklung der Ziele des Fremdsprachenunterrichts vom grammatischen Ansatz zur sprachlichen Kompetenz gespielt. Die Idee der Einheit einer Sprache und einer Kultur ist auch in den Arbeiten von Erasmus Roterodamus, Jean-Jacques Rousseau und Gottfried Leibniz zu finden. Der Hauptmangel dieser Theorien besteht darin, dass sie „die Welt der Sachen“, d.h. die Kultur als eine universelle Erscheinung allgemein für alle Völker betrachteten und keine nationalen Subkulturen ausgliederten. Trotzdem hatten diese Theorien für die Entwicklung der Didaktik des Fremdsprachenunterrichts große Bedeutung.

In den pädagogischen Systemen des schweizerischen Pädagogen Johann Heinrich Pestalozzi und des russischen Pädagogen Konstantin Dmitrievich Uschinskij waren die nationalen Besonderheiten der Kulturen berücksichtigt. In ihren Ideen wurde sowohl die Notwendigkeit des gleichzeitigen Erlernens der Sprache und der Kultur, oder ,,der Sprache in der Kultur“ als auch die Bedeutung der fremden geistigen und materiellen Kultur für die Erziehungs- und Ausbildungszwecke betont.

„Gemeinsam mit Fähigkeiten des Lernenden und methodischen Unterrichtsverfahren bilden die Informationen über die fremde Kultur ein wichtiges Ziel des Lernprozesses. Der Lehrer muss nicht von den engen sprachlichen Zielen begrenzt werden. Ohne Heranziehen der Informationen über die fremde Kultur wird der Fremdsprachenunterricht ärmer, er wird bloß zur Aneignung der phonematischen, lexikalischen und grammatikalischen Erscheinungen. Man muss natürlich größeren Wert auf das Erlernen der Sprechmechanismen und das Training der Sprachmodelle legen, aber der Lehrer darf auf keinem Fall die goldene Regel vergessen: man braucht keinen Fremdsprachunterricht, wenn es nichts zu sagen gibt“" (Lassera 1974, S. 218).

\footnotetext{
Hier und im Folgenden ist immer auch die weibliche Form mit einbezogen.
} 
Damit wurde der kulturelle Wert des Lehrfaches anerkannt. „Der Inhalt der Sprache ist mit der Kultur unzertrennlich verbunden. Die Sprache in ihrem Wortschatz spiegelt die Kultur wider, welche sie bedient" (Sepir 1993, S. 194).

Unterstützt von der Idee der Kontinuität einer Sprache und einer Kultur hat die kulturelle Komponente Einstieg in die Zielsetzung des Fremdsprachenunterrichts gefunden. Anfangs als eine der Komponenten der sprachlichen Kompetenz ausgegliedert, gewann sie im Laufe der Zeit mehr an Bedeutung, bis sie letztendlich ebenso wie Wortschatz, Phonetik und Grammatik zu den wichtigsten Bestandteilen des Fremdsprachenunterrichts geworden ist. In der Zeit, in der eine Verschiebung der Schwerpunkte im Unterricht von der grammatikalischen Kompetenz zur Sprachkompetenz zu beobachten ist, entsteht eine Wissenschaft, die sich mit der kulturellen Komponente befasst. Sie wurde zuerst Kulturkunde, später dann Landeskunde genannt. Das Ziel der Kulturkunde, die am Anfang des 20. Jahrhunderts die lexikalisch-grammatikalische Arbeit im Fremdsprachenunterricht begleitet hat, war die Aneignung des ,authentischen, sogar enzyklopädischen Wissens über die Realien ${ }^{4}$ der Kultur, der Geschichte und der Gesellschaft des Landes der Fremdsprache“ (Lukjantschenkowa 1998, S. 27). Solche Informationen wurden durch verschiedene Sachtexte zu geografischen, historischen oder gesellschaftlichen Fakten präsentiert, die den Lernenden die Einsichten in die Sprachgemeinschaft des Ziellandes erlaubten. Oft bedeutete die kulturkundliche Information im Fremdsprachenunterricht zum Anfang des 20. Jahrhunderts einfach Information über das Zielland, die auch in der Muttersprache des Studierenden mitgeteilt wurde.

„Das Gegenkonzept der realienkundlichen Ausrichtung formierte sich zu Beginn des 20. Jahrhunderts als Kulturkunde und glaubte, in Auseinandersetzung mit literarisch anspruchsvollen Werken zur Wesensart des fremden Volkes vorzudringen“ (Gehring 2002, S. 74).

\section{Sprachkompetenz}

Der grammatikalische Ansatz dominierte bis Anfang des 20. Jahrhunderts. In den letzten Jahrzehnten des 19. und in den ersten Jahrzehnten des 20. Jahrhunderts wurde aber heftige Kritik an dem grammatikalischen Ansatz zum Erlernen einer Fremdsprache geübt.

Als Ziel des Fremdsprachenunterrichts trat die sprachliche Kompetenz in den Vordergrund. Die Übersetzung verlor ihre beherrschende Position in der Didaktik. In den Vordergrund

Realien sind Objekte, die nur für die bestimmte nationale Kultur charakteristisch sind, wie z.B. Trachten, Spezialitäten etc. 
traten aktive Arbeitsformen, bei denen reproduktive Fähigkeiten trainiert wurden. Die Übungen unterlagen folgendem Prinzip: Der Lernende muss mit Hilfe des Lernmaterials in zu der erlernenden Fremdsprache bestimmte Aussagen bilden können.

„Gelenkte Aktivübungen, solche, bei denen Lernende unter Zuhilfenahme von linguistischen Orientierungshilfen und metasprachlichen Impulsen reproduktive Äußerungen formulieren, haben neben der eng gesteuerten Beantwortung von Fragen zu Arbeitstexten den größten Anteil““ (Gehring 2002, S. 74).

Die Lehrmaterialien wurden aber nur auf einen Menschen ausgerichtet, d.h. es fehlten die Kommunikationspartner. Das bedeutet, obwohl die Schwerpunkte des Unterrichts von der „Grammatik“ in die Richtung „Sprechen“ verschoben wurden, blieb der Sprachunterricht trotzdem nicht kommunikativ. Durch die Übungen wurden bestimmte grammatikalische und lexikalische Formen trainiert, wobei unbeachtet blieb, welche Bedeutungen und Intentionen diese grammatischen oder lexikalischen Formen beinhalten. Die sprachliche Kompetenz war ein Schritt zum realen Sprachgebrauch; was aber den Kontext oder die Landeskunde angeht, blieben diese Bereiche außerhalb des Fremdsprachenunterrichts.

Bei der Zielsetzung ,sprachliche Kompetenz“ blieb die Situation im Wesentlichen unverändert, da die Bezüge zur kulturellen Realität weiterhin aus dem Klassenunterricht ausgeklammert wurden. Die zu bearbeitenden Sachtexte, die zwar mit dem neuen Etikett „landeskundliche Informationen“ versehen wurden, berichteten ebenso wie früher über die Sachverhalte des Ziellandes. Es bestand zwar der Versuch, mehr Einsichten in das Alltagsleben der fremden Sprachgemeinschaft durch die Informationen über typische Charaktereigenschaften oder Wertvorstellungen der Bürger des fremden Landes zu geben, dies geschah aber meistenteils so verallgemeinert, dass es nur zur Entstehung oder Bestätigung von Stereotypen führte. Die möglichen realen Interaktionsintentionen des Sprachlernenden und seiner fremdsprachlichen Partner blieben außerhalb des Interessenbereichs des Unterrichts, also unbeachtet.

\section{Kommunikative Kompetenz}

Die 60er und 70er Jahre des 20. Jahrhunderts sind in der Didaktik des Fremdsprachenunterrichts durch einen neuen Ansatz zum Verstehen des Unterrichtsziels gekennzeichnet. Die große Bedeutung der zu erlernenden Sprache für die Kommunikation wurde erkannt, deshalb wurde der neue Ansatz als ,kommunikativer Ansatz“ bezeichnet, und Kommunikationsfähigkeiten wurden zu Zielen des Fremdsprachenunterrichts erklärt. Der Schwerpunkt des Unterrichts lag nicht mehr bei den Sprachstrukturen und dem formalen Gebrauch der 
Sprache, sondern wurde auf ihren Gebrauch als Kommunikationsmittel gelegt. Es wurde anerkannt, dass die korrekte Beherrschung des Sprachsystems allein nicht ausreichend ist, um eine erfolgreiche Kommunikation und gegenseitiges Verständnis der Gesprächspartner zu erzielen.

„Das Sprachverhalten, das nur damit zufrieden ist, eine Aussage mit der richtigen linguistischen Form bekleiden zu können, kann den Normen der kommunikativen Kompetenz nicht entsprechen. Eine Aussage ist nur dann adäquat, wenn sie im Kontext eingegliedert ist und die Form besitzt, die dem gegebenen Diskurs entspricht“" (Gehring 2002, S. 78).

Die kommunikative Kompetenz als Ziel des Fremdsprachenunterrichts ist eine Voraussetzung für die Produktion der situationsbedingten Rede, wo „es wichtig ist, dass der Lernende sich die von ihm gelernten Sprachmodelle in Zusammenhang mit der konkreten Situation, d.h. in der konkreten fremden Kultur vorstellen konnte“ (Buttjes 1995, S. 143). Eine der bekanntesten Methoden des Unterrichts innerhalb dieser Zielsetzung war die sogenannte audiolinguale Methode, die in der Fremdsprachendidaktik der USA entwickelt wurde. Das Grundelement dieser Methode wurde als patterns practice bezeichnet, mit deren Hilfe imitativ-reaktive Sprechanlässe geschaffen wurden, deren wiederholter Gebrauch den Lernenden zur gewohnheitsmäßigen Verwendung der Aussagemuster verhelfen soll, ohne grammatische Regeln bewusst zu machen.

Der kommunikative Ansatz berücksichtigt die Beziehung zwischen den Sprachstrukturen und dem Diskurs, bzw. dem kulturellen Kontext. Die Sprache wird als Werkzeug betrachtet, mit deren Hilfe der Sprechende die kulturbedingten Faktoren wahrnehmen kann, damit zum Mittel für das Erkennen der fremden Kultur.

Mit dem kommunikativen Ansatz veränderten sich auch die Einstellungen gegenüber der Landeskunde und infolgedessen die Arbeitsweise mit den landeskundlichen Materialien. Gehring (2002) nennt als Hauptkomponenten der kommunikativen Kompetenz neben den sprachlichen und soziolinguistischen auch Diskurskompetenz und soziokulturelle Kompetenz.

Eine wichtige Rolle bei der Positionierung der kulturellen Komponente in der Hierarchie der Ziele des Fremdsprachenunterrichts hat die linguistische Landeskunde gespielt, die von den Linguisten Werestschagin und Kostomarov in den früheren 70er Jahren in der russischen Sprachdidaktik entwickelt wurde.

„Linguistische Landeskunde ist ein Aspekt des Unterrichtens der russischen Sprache für Ausländer, wo die kumulative Funktion der Sprache durch die landeskundlichen Informationen sowohl zum Ziel einer Kommunikation als auch für die Erfüllung allgemeinbilden- 
der und humanistischer Aufgaben des Unterrichts eingesetzt wird. Es findet beim Lernenden ein Akkulturationsprozess statt, durch den er die allgemeinen Erscheinungen der modernen Umwelt kennenlernt. (...) Das oben erwähnte Kennenlernen erfolgt innerhalb des Russischunterrichts und durch die russische Sprache“ (Werestschagin und Kostomarov 1983, S. 49).

In diesem Konzept wurde die unzertrennliche Einheit einer Sprache und derer Kultur hervorgehoben. Die Gründer des Konzepts haben die Aufmerksamkeit darauf gelenkt, dass schon in der Sprache selbst ein großes Potenzial des kulturellen Wissens enthalten ist. Sie sind der Meinung, es sei notwendig, Hintergrundkenntnisse und Wissen zu beachten, die hinter den sprachlichen Einheiten verborgen sind. Einen großen Wert legten die russischen Wissenschaftler auf die Entwicklung der Persönlichkeit des Lernenden im Prozess des Erlernens einer Fremdsprache, sodass der Lernende nach ihrer Hypothese einem der Sozialisation ähnlichen Prozess unterliegt, den sie als Prozess der Akkommodation bezeichnen. Darunter wird Einleben in die neue Kultur verstanden, im Laufe dessen die Merkmale der eigenen Kultur in die neue Kultur integriert werden können.

\section{Interkulturelle Kompetenz}

Seit dem Anfang der 1980er Jahre wird die interkulturelle Kompetenz, die die anthropologische Grundidee einbezieht, zum Ziel des Fremdsprachenunterrichts. Das bedeutet eine besondere Achtung sowohl der Positionen des Lernenden/des Sprechenden als auch der Positionen seines Gesprächspartners. Im Vergleich zu der kommunikativen Kompetenz wird der Prozess der Entwicklung der interkulturellen Kompetenz breiter und tiefer gefasst. Während die kommunikative Kompetenz den Gebrauch der richtigen Sprachkonstruktion und die dem Diskurs entsprechenden Aussagen hervorhebt, sorgt die interkulturelle Kompetenz dafür, dass die Aussagen ,der konkreten Lebenssituation, den wahren Traditionen, Bräuchen, der Mentalität, sogar den Gewohnheiten und den Einstellungen als auch dem alltäglichen Verhalten und den nonverbalen Kommunikationsformen, die für die Kultur des Ziellandes üblich sind, entsprechen“ (Serebrjannikowa 2000, S. 101).

Obwohl sich die Didaktiker des Fremdsprachenunterrichts schon seit mehr als 20 Jahren mit dem interkulturellen Ansatz befassen, herrscht kein einheitliches Bild. Man kann allerdings zwei Richtungen dieses Ansatzes unterscheiden: eine sachorientierte und eine schülerorientierte. Erstere, die besonders in den 80er Jahren verbreitet war, vertritt die Meinung, dass die Vermittlung einer fremden Zielkultur im Mittelpunkt des Unterrichts stehen soll. Interkulturelle Kompetenz wird hier als „Ergänzung der fremdsprachlichen Kompe- 
tenz durch landeskundliches Orientierungswissen“ verstanden, „dessen Schwerpunkt auf der Kenntnis und Beherrschung gesellschaftlicher Praktiken liegt“ (Eckerth und Wendt 2003, S. 10). Für die Auswahl von Materialien ist es hier wichtig, dass sie Informationsträger über die Zielkultur sind, d.h. sich für die Erschließung der jeweils fremden Kultur eignen.

Aus systematischer Sicht geht es dabei um:

1. Vermittlung von Kenntnissen über die Oberflächenphänomene von Kulturen und von Einsichten in ihre Tiefenstrukturen, die die Oberflächenphänomene determinieren,

2. Erzeugung eines Problembewusstseins für die Prozesshaftigkeit kultureller Erscheinungen durch die Einbeziehung der historischen Dimension;

3. Vermittlung von Einsichten in die Vernetzung von Kulturen, bzw. Subkulturen mit den sie umgebenden Lebenswelten als Auslöser des ständigen Wandels,

4. Schaffung eines Bewusstseins für die Verschiedenartigkeit, Einzigartigkeit und Begrenztheit von fremden Kulturen (vgl. Einhoff 1993, S. 250).

Die sachorientierte Richtung setzt im Großen und Ganzen die Traditionen des kommunikativen Ansatzes fort, wobei kommunikative Kompetenz als Ziel verstanden wird, mit einer besonderen Hervorhebung der Landeskunde.

Die zweite schülerorientierte Richtung des interkulturellen Ansatzes liegt dem Konzept dieser Arbeit zugrunde. Die Verfasserin versteht den Fremdsprachenunterricht als einen Ort, wo zwei kulturelle Welten aufeinander treffen. Bei diesem Prozess ist es wichtig, durch eine kontrastive Gegenüberstellung von zwei kulturellen Erscheinungen die Unterschiede und Zusammenhänge der fremden und eigenen Kultur wahrzunehmen, was einerseits das eigene Verhalten innerhalb der Zielkultur adäquat macht, andererseits gegenüber eigenen kulturellen Einstellungen sensibilisiert und eigenkulturelle Identität stärkt. Dabei tritt die einfache Vermittlung des landeskundlichen Wissens in den Hintergrund, in den Vordergrund treten dafür Themen und Materialien, um die „Unterschiede und Gemeinsamkeiten zwischen Zielkultur und eigenen Kultur zu erkennen“ und „zum adäquaten Verhalten in der Begegnung mit den Menschen aus fremden Kulturen zu befähigen. Darüber hinaus wird Kriterien wie sprachliche Kraft, Aktualität und Lebenshilfe ein besonderer Stellenwert eingeräumt“" (Einhoff 2003, S. 8).

Die oben diskutierten Kompetenzen (grammatikalische, sprachliche, kommunikative und interkulturelle) sind in den Zielsetzungen des Fremdsprachenunterrichts verankert. Die Verfasserin ist der Ansicht, dass zukünftig zwei weitere Kompetenzen im Fremdsprachen- 
unterricht entwickelt werden sollten: multikulturelle und transkulturelle Kompetenz.

\section{Multikulturelle Kompetenz}

Dieser Ansatz ist als eine Weiterentwicklung des interkulturellen Ansatzes zu verstehen. Die Vertreter dieses Ansatzes sind der Meinung, dass es in der modernen Welt kaum nationale Gesellschaften mit einer homogenen Kultur gibt. Jede nationale Kultur ist in mehrere Subkulturen unterteilt, die zwar häufig einige Unterschiede aufweisen, „die aber letztlich ein in sich geschlossenes, relativ autonomes und daher einzigartiges Gebilde darstellt und ihre Mitglieder im Sinne eines kulturspezifischen Verhaltens prägt" (Einhoff 2003, S. 9). „The multicultural approach includes a specific focus on the ethnic und linguistic diversity of the target country or countries, e.g. Bretons and Moroccans and indigenous French in France. It also deals with the relation between target countries and the learner's own, and other countries, including migratory relations (where do immigrants and refugees come from, and why?). The teaching may include comparisons between the target countries and the learners' own, thereby inviting learners to develop a reflective attitude to the cultures of their own country. The teaching may reflect wishes for a balanced and anti-racist view of cultures involved" (Risager 1997, S. 246).

Wie aus dem angeführten Zitat zu ersehen ist, ist der multikulturelle Ansatz genauso wie der interkulturelle auf die Lernerperspektive ausgerichtet. Der multikulturelle Ansatz ergänzt und erweitert zwar den interkulturellen, multikulturelle Kompetenz kann aber m.E. nicht oberstes Ziel des Fremdsprachenunterrichts sein.

\section{Transkulturelle Kompetenz}

Der transkulturelle Ansatz übt gegenüber dem interkulturellen auch Kritik am Kulturbegriff. Während der multikulturelle Ansatz großen Wert auf die Tatsache legt, dass sich die ehemaligen Großkulturen in ein Nebeneinander vieler Partikularkulturen auflösen, befasst sich der transkulturelle Ansatz mit folgender Erscheinung: Durch Migration, moderne Kommunikationssysteme und internationale Wirtschaft werden in der letzten Zeit starke Globalisierungsprozesse ausgelöst, die auch dafür verantwortlich sind, dass die einzelnen Kulturen nicht geschlossen und unabhängig bleiben können. Diese Prozesse führen zur Verschmelzung verschiedener Kulturen, wodurch auf einer neuen Ebene eine neue Weltkultur entsteht, die ,alten“ Nationalkulturen aber durch fremde Einflüsse zu Hybriden werden. Diese Erscheinung wird Transkulturalität genannt.

Gestützt auf diese Tatsachen kommt auch die transkulturelle Kompetenz als Ziel des 
Fremdsprachenunterrichts in Frage.

„The transcultural approach focuses on the life of individuals and groups in contexts characterized by more or less cultural and linguistic complexity. The teaching deals not only with the traditional target countries, but also with other countries, areas or cultural contexts, if this may contribute to language learning" (Risager 1997, S. 248).

Als ideales Ziel schlägt Risager Mediatorenkompetenz vor, die gleichzeitig interkulturelle und kommunikative Kompetenz einschließt. Er legt aber einen anderen Schwerpunkt, und zwar: diese Kompetenz muss die Fähigkeit darstellen, die Sprache als Kommunikationscode in allen Situationen bei jeglichem kulturellen und linguistischen Schwierigkeitsgrad einsetzen zu können. Die transkulturelle Kompetenz soll helfen, die Probleme, die durch Globalisierung und Verflechtung der Kulturen verursacht werden, auf einer neuen transkulturellen Ebene zu lösen.

Transkulturelle Kompetenz ist sicherlich eine wichtige Zielsetzung, die die Traditionen der interkulturellen und weiterer kultureller Erscheinungen übernehmen kann. Sie sollte jedoch nicht nur Ziel des Fremdsprachenunterrichts sein, sondern fächerübergreifend realisiert werden. Transkulturelle Kompetenz sollte zu einem der obersten Ziele des Lernens werden, damit die Lernenden, sei es in einer Schule, an einer Universität oder in einer anderen Lehreinrichtung, auf das Leben in einer mehr und mehr globalisierten Welt vorbereitet werden. Der Fremdsprachenunterricht muss dabei auch ein Teil dieses Lernens sein mit eigener Zielsetzung, die diesem oberen Ziel untergeordnet ist.

Ein weiterer Punkt, der zu überlegen ist, ist die Sprache selbst. Wenn Risager (1997) in seinem Artikel „Language teaching and the process of European integration“ über transkulturelle Kompetenz als Ziel des Fremdsprachenunterrichts spricht, bezieht er sich auf die englische Sprache; oft schreibt er auch über die Bedeutung der Sprache als lingua franca. Da die vorliegende Arbeit sich aber mit der deutschen Sprache und deren Vermittlung im Fremdsprachenunterricht beschäftigt, steht die transkulturelle Kompetenz nach Meinung der Verfasserin nicht im Mittelpunkt. 


\subsection{Zur aktuellen Diskussion von Begriffen aus der modernen Fremdsprachendidaktik}

Im folgenden Kapitel setze ich mich mit einigen Begriffen auseinander, die in der Fremdsprachendidaktik häufig verwendet werden. Eine besondere Aufmerksamkeit wird dabei den Begriffen kommunikative und interkulturelle Kompetenz gewidmet.

„Am wichtigsten ist mir, dass meine Schüler ihre Vorurteile aufbrechen und Menschen aus anderen Ländern nicht als Angehörige einer Nation, sondern als Menschen wahrnehmen und akzeptieren“ (Caspari 1997, S. 70). Diese Aussage stammt von einer Deutsch- und Englischlehrerin mit 21 Jahren Berufserfahrung und führt ein in die Diskussion zur aufgeworfenen Frage nach der Bedeutung unterschiedlicher Kompetenzen für das Erlernen einer Fremdsprache. Was für eine praktizierende Lehrerin in einfachen Worten auszudrücken ist, stellt für die wissenschaftliche Literatur ein weites Feld für Diskussionen dar. Die schon im vorigen Kapitel erwähnten Ziele wie kommunikative, interkulturelle, multikulturelle und transkulturelle Kompetenzen sind in unterschiedlichen Kontexten in der wissenschaftlichen Literatur zu finden, ebenso werden Fremdverstehen, interkulturelles Verstehen, interkulturelle Landeskunde als Zielsetzungen genannt.

Diese Vielfalt ist dadurch zu erklären, dass moderne Sprachdidaktik unter dem Einfluss von mehreren anderen verwandten Disziplinen steht. So nehmen laut Roche (2001) die folgenden Disziplinen eine Grenzstellung zu der modernen Fremdsprachendidaktik ein: Linguistik und ihre Teildisziplinen, besonders Pragmatik, Soziolinguistik, kognitive Linguistik; Sprachpädagogik; Interkulturelles Training; Psycholinguistik, Psychologie, Neurowissenschaften; Geisteswissenschaften wie Hermeneutik, Literaturwissenschaft, Philosophie; Sozialwissenschaften und Kulturwissenschaften: Soziologie, Anthropologie, Politikwissenschaft, Geschichtswissenschaft; Wirtschaftswissenschaften; Jura; Naturwissenschaften; Ingenieurwissenschaften.

Im Weiteren werden einige Ansätze präsentiert, die sowohl aus den erziehungswissenschaftlichen als auch aus den fremdsprachendidaktischen Konzepten stammen und einen großen Einfluss auf die Begriffsdefinition der interkulturellen Kompetenz ausüben. Es handelt sich um die Ansätze:

- Fremdverstehen

- Interkulturelles Verstehen

- Kommunikative Kompetenz

- Interkulturelle Kompetenz 
Unter dem Einfluss der interkulturellen Hermeneutik, die sich mit den Problemen der Auseinandersetzung zwischen dem Eigenen und dem Fremden befasst, hat „Fremdverstehen“ einen Zugang in die Zielsetzungen des Fremdsprachenunterrichts gefunden. Das Verstehen rückt in den Mittelpunkt und wird nicht nur als Prozess, sondern auch als Ziel verstanden. Das Fremde wird hier als eine Kategorie betrachtet, die angeeignet werden soll. Dies kann durch das Erwerben von Wissen über „das Fremde“ geschehen, das aber auch zur Annäherung an die Perspektive des anderen führen soll. Dieser Prozess wird durch Selbstreflexion begleitet. Nur durch Aneignung und Verstehen des Fremden kann das Eigene auch verstanden und reflektiert werden. Fremdverstehen ist eine gegenseitige Bereicherung von zwei oder mehreren Kulturen, die dabei die Einzigartigkeit und die eigene Prägung nicht verlieren (vgl. Serebrjannikowa 2000, Caspari 1997, Kallenbach 1997). Wichtig ist dabei, dass der Verstehensvorgang als Kommunikationsprozess verstanden wird.

Einen weiteren Begriff finden wir bei Vollmer. Er zieht den Begriff „,interkulturelles Verstehen“ vor und versteht darunter die „Fähigkeit zur kommunikativen Verständigung unter den Bedingungen von Zwei/Mehrsprachigkeit mit Angehörigen einer bestimmten Kultur oder beliebiger anderer Kulturen“ (Vollmer 1994, S. 175-176). In diesem Konzept wird ein Schwerpunkt nicht nur auf Fremdverstehen, sondern auch auf Eigenverstehen gelegt. Die Aufgabe des Fremdsprachenunterrichts sieht er in dem kontrastiven Vergleich der beiden kulturellen Erfahrungen: die erste stellt das Ergebnis einer Kommunikation mit einem Fremden dar, die zweite ist die Selbsterfahrung. Die Auswertung beider Erfahrungen soll zum Aufbau des interkulturellen Verstehens führen, ,weil sich doch gerade im Medium der Sprache, in der Begegnung mit einem fremdsprachigen Sprecher oder Text, durch Aneignung und Aushandlung soziokultureller Bedeutung eigene wie fremde Erfahrung besonders gut aufeinander beziehen und miteinander vermitteln lassen“(Vollmer 1995, S. 105).

Das Ziel „Fremdverstehen“ wurde auch seitens der Literaturdidaktik unterstützt, obwohl wir bei dieser Wissenschaft keine einheitliche Meinung darüber finden. ${ }^{5}$ Die Literaturdidaktik, die den Begriff „Verstehen“ vorzieht (vgl. Bredella 1993, 1994), beschäftigt sich mit dem Informations- und Entwicklungspotenzial von literarischen Texten, die im Fremdsprachenunterricht angeboten werden. Durch die Auseinandersetzung mit diesen Texten sollen die Lernenden nicht nur die fremden Kontexte kennenlernen, sondern sich auch mit diesen vertraut machen. Im Unterschied zu der interkulturellen Landeskunde liegt der

\footnotetext{
In den Arbeiten von Literaturdidaktikern sind unterschiedliche Formulierungen des Ziels des interkulturellen Lernens zu finden. Es hat nach der Meinung der Verfasserin damit zu tun, dass diese Wissenschaft keine abgegrenzten Räume für die Realisierung des interkulturellen Ansatzes hat und im Fremdsprachenunterricht als untergeordnete Teildisziplin integriert ist.
} 
Schwerpunkt auf Fremdverstehen. „Anders als in der Landeskundedidaktik, wo Perspektivenkoordination angestrebt wird, möchte das Konzept des Fremdverstehens den temporären Perspektivenwechsel erreichen“ (Gehring 2002, S. 71).

Einen weiteren Anstoß bekam der Fremdsprachenunterricht von Wissenschaften, die sich mit den Problemen der Konfrontation zwischen den Kulturen auseinandersetzten, insbesondere mit denen in einer multikulturellen Gesellschaft oder während kurzer Aufenthalte von Vertretern einer Kultur in einem anderen Kulturkreis. Zu solchen Wissenschaftszweigen gehört die Ausländerpädagogik mit ihrer Nachfolgedisziplin „Interkulturelle Pädagogik“, die im deutschsprachigen Raum entwickelt wurde. Aber auch die sogenannten Cultural Studies, die englischen und amerikanischen Kulturwissenschaften, befruchteten mit ihren Ideen und Theorien die Fremdsprachendidaktik. Da die Kultur in den Cultural Studies der 80er Jahre neu definiert wurde, wurden die zusätzlichen kulturellen Informationen in den Unterricht integriert. Ihr Schwerpunkt lag nicht nur z.B. auf Geografie, Geschichte oder Politik, sondern das Alltagswissen, also die Kultur als Alltagspraxis, stand im Mittelpunkt. Für den Unterricht bedeutete die Berücksichtigung dieser thematischen Schwerpunkte eine Erweiterung des Curriculums. Während die statistischen Angaben über das Zielland und seine Traditionen und Bräuche früher als Vermittler der fremden Kultur galten, rücken nun die Informationen über Einstellungen des alltäglichen Lebens in den Vordergrund: z.B. wie in der Zielgesellschaft mit Zeit oder Raum umgegangen wird, ob individuelle oder kollektive Einstellungen den Vorrang haben ${ }^{6}$.

Während die im US-amerikanischen Raum entstandenen Cultural Studies ursprünglich als Gesellschaftsforschung in Bezug auf Alltagskultur versus Hochkultur gedacht waren, musste sich die Ausländerpädagogik in Deutschland schon mit den Problemen einer zunehmend multikulturellen Gesellschaft auseinandersetzen. Die Ausländerpädagogik, in der die sprachlichen Defizite und die von den deutschen „Normen“ abweichenden kulturellen Einstellungen als Mangel betrachtet werden, entwickelte sich zur interkulturellen Pädagogik, welche die multikulturelle Gesellschaft als einen Ort der persönlichen Bereicherung sieht. Jedes Mitglied dieser Gesellschaft soll eine erfolgreiche Kommunikation mit anderen Bürgern führen können und nicht nur gegenüber den „fremden“ Werten sensibel sein, sondern auch die eigene kulturelle Prägung reflektieren. Als Schlüsselkompetenz für die Mitglieder einer multikulturellen Gesellschaft wurde interkulturelle Kompetenz benannt. Auernheimer definiert interkulturelle Kompetenz als die Fähigkeit, kulturelle Bedeutungen und Ver-

Diese Kategorien sind aus den Kulturtheorien von Hall (1989) und Hofstede (1993) entnommen. Weitere Vertreter der Cultural Studies sind Thompson (1990), Bourdieu (1985) und andere. 
haltenserwartungen auszuhandeln, dabei tolerant und sensibel gegenüber dem fremden Partner zu sein und die kulturelle Selbstreflexion als wichtig anzusehen (vgl. Auernheimer 2005, S. 18; Auernheimer 2003). Obwohl der Personenkreis von den Migranten auf die gesamte Bevölkerung einer multikulturellen Gesellschaft ausgedehnt wurde, blieben die Ausländer oder die Mitbewohner mit Migrationshintergrund im Mittelpunkt des Interesses der interkulturellen Pädagogik. Eine Zentralstellung nimmt nach wie vor die Frage nach ihren Kenntnissen der deutschen Sprache ein. Die Grundprinzipien und Konzepte, aber auch Aufgaben und Ziele der interkulturellen Erziehung fanden ihren Niederschlag in der Praxis des Unterrichts „Deutsch als Zweitsprache“ und wurden später auch in den Unterricht „Deutsch als Fremdsprache“ übertragen. Der aktuelle Stand ist folgender: Dieses Gedankengut, das zuerst in Bezug auf die Vermittlung einer Fremdsprache in multikulturellen Gesellschaften, z.B. den USA oder Deutschland, gesammelt wurde, diente als Anregungsmaterial für die Didaktiker, was auch zu seiner Übernahme in die modernen Konzepte des Deutschunterrichts im Ausland geführt hat (vgl. Werestschagin und Kostomarov 1983, Serebrjannikowa 2000).

Durch die Erweiterung des Konzepts „Landeskunde“ und durch seine Integration in die Sprachdidaktik wurde das eigentliche Ziel „kommunikative Kompetenz“ oder Kommunikationsfähigkeit in die transnationale Kommunikationsfähigkeit umbenannt. Erst später erscheint in landeskundedidaktischen Arbeiten der aus der interkulturellen Pädagogik ausgeliehene Begriff „interkulturelle Kompetenz“6.

In der wissenschaftlichen Literatur wird über einen interkulturellen Ansatz oder über die interkulturelle Landeskunde gesprochen. Während bis Anfang der 90er Jahre möglichst vollständiger Wissensstoff über die fremde Kultur vermittelt werden sollte, sieht interkulturelle Landeskunde, die zwar auch für die kulturellen Informationen in der Sprache verantwortlich ist, ihre Aufgabe in dem Aufbau eines Orientierungssystems, das die unterschiedlichen Normen und Werte der eigenen und der fremdkulturellen Sprachgemeinschaft als mögliche Ursachen eines Missverständnisses zu reflektieren hilft. Das heißt, dass nicht nur kulturspezifisches Wissen, sondern in erster Linie die Entwicklung der Einstellungen, Fähigkeiten und Fertigkeiten im Umgang mit fremden Kulturen Vorrang haben. Dabei stehen ihr die anderen zwei Aspekte der Landeskundedidaktik zur Seite, und zwar ein sprachbezogener Aspekt, welcher Informationen über Geografie, Geschichte und einige Erscheinungen des Alltags vermittelt, und ein problembezogener Aspekt, der auf Diskurskompetenz zielt und Interpretations- und Reflexionsfähigkeit als Hilfen bei der Lösung von Kommunikationsproblemen mit den fremdsprachlichen Partner zu entwickeln versucht (vgl. Buttjes 
1991, 1995).

Interkulturelle Landeskunde hat jedoch nicht nur Lernziele auf der Ebene der Einstellungen und Haltungen, wie im Folgenden zu zeigen sein wird, sondern es werden auch Lernziele auf den anderen Lernzielebenen (wie Kenntnisse, Einsichten und Fertigkeiten) neu interpretiert. Darüber hinaus sind Stoffauswahlkriterien sowie spezielle methodische Verfahren für interkulturelles Lernen durchaus vorhanden, sodass es berechtigt ist, von einer eigenständigen interkulturellen Landeskunde zu sprechen, die die verwendbaren Aspekte kognitiver oder kommunikativer landeskundlicher Ansätze gewissermaßen ,aufhebt“ und unter interkulturellen Vorzeichen weiterführt (vgl. Zeuner 1997, S. 4).

In der Fremdsprachendidaktik lassen sich drei Hauptdiskussionsrichtungen unterscheiden. Erstens die aus der Hermeneutik und Literaturwissenschaft angestoßene Diskussion über die gegenseitige Einflussnahme und Abhängigkeit des Eigenen und des Fremden. Aus dieser Diskussion sind solche Begriffe wie Fremdverstehen oder interkulturelles Verstehen in die Fremdsprachendidaktik eingeflossen. Da es bei dieser Diskussion immer um zwei sich gegenüberstehende Wertesysteme geht, hat hier die kontrastive Methode als Hauptarbeitsmethode Priorität, wobei es diese zwei Systeme zu vergleichen gilt.

Die zweite Diskussionsquelle stammt aus den Wissenschaften, die sich mit Problemen bei interkulturellen Kontakten beschäftigen, und zwar aus den aus dem amerikanischen Raum stammenden Cultural Studies und aus der deutschen Ausländerpädagogik. Zu den Grundprinzipien dieser Wissenschaften gehören das neue Verständnis der Kultur und die Erziehung $\mathrm{zu}$ bestimmten Charaktereigenschaften oder Einstellungen. Diese Grundprinzipien haben die Diskussion um die Vorgehensweise und den Wert des Fremdsprachenunterrichts beeinflusst, was besonders deutlich in der russischen Fremdsprachendidaktik zu sehen ist, da hier der Unterrichtsprozess in erster Linie als Erziehungsprozess und erst dann als Wissensvermittlungsprozess angesehen wird. Die Verfasserin hat für den Titel ihrer Arbeit den Begriff ,,interkulturelle Kompetenz“ ausgewählt, der aus dieser Diskussion stammt.

Die dritte Diskussionsquelle der interkulturellen Ideen hat ihren Ursprung in der Fremdsprachendidaktik selbst und ist mit einem neuen Verständnis der Landeskunde verbunden. Im Rahmen dieser Diskussion wird die allgemeine Kommunikationsfähigkeit als Ziel des Fremdsprachenunterrichts durch die interkulturelle Funktion der landeskundlichen Materialien erweitert. Hier sind Begriffe wie „transnationale Kommunikationsfähigkeit“" und „interkulturelle Kompetenz“ gebräuchlich. Ein weiterer Begriff, der auch sehr häufig in den Arbeiten zu finden ist, ist die ,interkulturelle kommunikative Kompetenz“. Oft wird das Wort „interkulturell“ in Klammern gesetzt, als ob der Autor nicht entscheiden könnte, 
ob die interkulturelle Seite der kommunikativen Kompetenz besonders hervorgehoben werden sollte. Es scheint, dass für einige Autoren kein wesentlicher Unterschied zwischen der kommunikativen und der interkulturellen Kompetenz besteht (vgl. z.B. Knapp-Potthoff 1997, Marenkowa und Novikowa 2001, Gick 1997).

Bevor im Weiteren die interkulturelle Kompetenz als ein selbstständiges und angemessenes Ziel des Fremdsprachenunterrichts betrachtet werden kann und der Zusammenhang mit der kommunikativen Kompetenz diskutiert wird, sollen drei wichtige Merkmale des interkulturellen Lernens genannt werden, welche sich aus den oben angeführten Diskussionen ergeben und die bei Ausdifferenzierung der Kompetenzen weiterhelfen können:

1. Interkulturelles Lernen hat einen zweiseitigen Charakter. Die beiden Seiten, das Eigene und das Fremde, stehen in einem engen und einander beeinflussenden Verhältnis, deren Ziel die interkulturelle ${ }^{7}$ Verständigung ist.

2. Interkulturelles Lernen hat ein neues Verständnis von Kultur entwickelt, das von der klassischen Vorstellung der Kultur als „Veredlung der Sitten“ oder „höhere Lebensgestaltung“ (Flechsig 2001) der Menschen in Richtung einer Alltagskultur rückt, was sicherlich seine Auswirkung auf das dritte Merkmal hat.

3. Interkulturelles Lernen ist menschenorientiert, d.h. die Persönlichkeitserziehung, beinhaltet von Einstellungen und Werten, welche die Entwicklung von Fähigkeiten in sich einschließt und sogar voraussetzt, hat Vorrang vor der Wissensvermittlung, was den Lernprozess auf eine ganz andere Ebene bringt.

Die Gegenüberstellung der kommunikativen und interkulturellen Kompetenzen und die Diskussionen darüber in der wissenschaftlichen Literatur begannen um die Jahrhundertwende, als die interkulturelle Kompetenz als Ziel des Fremdsprachenunterrichts erkannt wurde. „Der Begriff ,interkulturelle Kompetenz“ hat seinen Vorgänger, die ,kommunikative Kompetenz', als neues globales Ziel für den DaF-Unterricht fast verdrängt und ist zu einem ebenso sinnentleerten Modewort verkommen“ (House 1996, S. 1). Die Meinung von Juliane House wird von einigen Didaktikern wie Knapp-Potthoff (1997), Knapp und Knapp-Potthoff (1990), Gick (1997) geteilt, während Autoren wie Vollmer (1994) oder Baxter (1983) nicht zustimmen. Was eigentlich ist unter der kommunikativen Kompetenz $\mathrm{zu}$ verstehen, und was wird an diesem Konzept kritisiert?

Folgende Definition war von dem Anthropologen und Soziolinguisten Dell Hymes (1972) entwickelt worden, dieser Ansatz wurde später in den Arbeiten von Canale und Swain (1980) Canale (1983) fortgesetzt. Hymes definiert die kommunikative Kompetenz als „ei-

\footnotetext{
Das Wort „inter“ setzt schon das Vorhandensein von mindestens zwei Seiten voraus.
} 
nes Sprechers und Lerners grammatisches psycholinguistisches, soziokulturelles und praktisch verfügbares Wissen und eine dementsprechende Gebrauchsfähigkeit dieser Sprache“. Obwohl diese Definition nur kognitive und Verhaltensebenen der Kompetenz beinhaltet und die affektive Seite außer Acht lässt, schließt sie dennoch innerhalb der Wissensebene ein breites Spektrum von Teilkompetenzen ein: sowohl diejenigen, die in dem rein sprachlichen Bereich liegen, als auch diejenigen, die außersprachliche, d.h. soziale Seiten betreffen. Im engeren Sinne ist die kommunikative Kompetenz eine durch extralinguistische Kenntnisse und Fähigkeiten vervollständigte linguistische Kompetenz. Diese zwei Teile finden wir auch in der Definition der russischen Didaktikerin Zernizkaja (2005):

„Kommunikative Kompetenz ist eine Gesamtmenge von automatisierten oder nicht automatisierten, wahrgenommenen oder nicht wahrgenommenen linguistischen oder extralinguistischen Kenntnissen oder Fähigkeiten, die zum Zweck des Verstehens der empfangenen Informationen oder der Produktion eines schriftlichen oder mündlichen Textes einzusetzen sind“ (Zernizkaja 2005, S. 48).

Es ist wichtig zu betonen, dass Kommunikation für jede Art von menschlichen Kontakten charakteristisch ist, und zwar in erster Linie in der Muttersprache und erst später in Bezug auf die Kontakte mit Vertretern der anderen Sprachgemeinschaften. Im Unterschied zu den später als offiziell angenommenen Bestandteilen der kommunikativen Kompetenz (KK) spricht die russische Autorin nicht von einer allgemeinen $\mathrm{KK}$, sondern von einzelnen kommunikativen Fähigkeiten, und zwar KK von Lesen, KK von Schreiben, KK von Hörverstehen und KK von Sprechen. Das Niveau der Entwicklung dieser Kompetenzen kann zwar unterschiedlich sein, aber es wird besonders unterstrichen, dass alle Arten von kommunikativen Texten eine wichtige Rolle spielen und dass die gute Beherrschung von nur einer Art der Kommunikation (z.B. nur schriftliche KK) nicht zur allgemeinen kommunikativen Kompetenz führen kann (vgl. Zernizkaja 2005, S. 49). Die Fähigkeit des Sprechens wird oft gegenüber den anderen Fähigkeiten hervorgehoben, und die kommunikative Kompetenz wird als eine innere Bereitschaft und Fähigkeit zum Sprechen verstanden (vgl. Nevirko 2003). Dabei werden die anderen kommunikativen Handlungen wie Schreiben, Hören und Lesen oft außer Acht gelassen.

Am häufigsten wird die kommunikative Kompetenz mit Hilfe von Teilkompetenzen beschrieben. Dabei existieren mehrere Klassifikationen. Hier soll auf die von Van Ek (1975) ausgearbeitete Theorie eingegangen werden, da sie auch in der Arbeit des Europarates genutzt wurde. Nach Van Ek schließt die kommunikative Kompetenz folgende Teilkompetenzen ein: 
- linguistische Kompetenz. Dazu gehören Kenntnisse in Wortschatz und Grammatik sowie die Fähigkeit, diese Kenntnisse einzusetzen;

- soziolinguistische Kompetenz. Dazu gehört die Fähigkeit, die Sprachstrukturen gemäß der Situation oder im Kontext zu benutzen;

- Diskurskompetenz. Das ist die Fähigkeit, einzelne Aussagen zu verstehen und logisch zu konstruieren, um produktiv an einer sinnvollen Kommunikation teilnehmen zu können;

- stratetegische Kompetenz. Sie beinhaltet die Fähigkeit, jene verbalen und nonverbalen Strategien zu benutzen, die den Mangel an Kenntnissen und Fähigkeiten in anderen Bereichen kompensieren können;

- soziokulturelle Kompetenz. Sie wird als Fähigkeit, sich im soziokulturellen Kontext zurechtzufinden, definiert;

- soziale Kompetenz. Sie schließt sowohl den Wunsch und die Bereitschaft, mit anderen zu kommunizieren, als auch die Fähigkeit, den Kommunikationsprozess zu steuern, ein.

Diese Klassifikation ist auch deshalb erwähnenswert, weil sie die meisten Teilkompetenzen nennt, darunter auch soziokulturelle und soziale, also diejenigen, die der Kulturkomponente eine besondere Bedeutung schenken. Es existieren aber auch andere Klassifikationen, die mit geringeren Abweichungen die Teilkomponenten benennen. Die berühmte Klassifikation von Dell Hymes (1972) nennt nur vier Gruppen von Kompetenzen, und zwar linguistische, soziolinguistische, diskursive und strategische Kompetenzen (vgl. House 1996, Nevirko 2003).

Ohne im Weiteren auf die einzelnen Klassifikationen der kommunikativen Kompetenz einzugehen, muss diese in Bezug auf die drei Ebenen - Sprache, Diskurs und Anwendung im realen Kommunikationsprozess betrachtet werden. Die unterschiedlichen Teilkompetenzen spielen eine Rolle bei der erfolgreichen Kommunikation innerhalb der unterschiedlichen Anwendungsebenen: So sichern die linguistische oder grammatische Teilkompetenz das Vokabular oder die grammatischen Regeln in der sprachlichen Ebene, Diskurs- und zum Teil auch strategische Kompetenzen drücken sich aus als Fähigkeit, die Sprache zum Zweck einer gelingenden Kommunikation zu verwenden. Die dritte Ebene stellt die Anwendung der Sprache in unterschiedlichen realen Lebenssituationen dar. Dabei sind für die Kommunikation die Fähigkeiten in den soziolinguistischen, sozialen und soziokulturellen Bereichen relevant. 
Gerade die dritte Anwendungsebene, die sich auf eine Kultur mit ihren Einstellungen und Regeln hinorientiert, lässt die Kritiker des interkulturellen Ansatzes behaupten, dass die kulturelle Komponente, die eine besondere Bedeutung für diesen Ansatz hat, schon in die Zielsetzung „kommunikative Kompetenz“ eingeschlossen ist. So setzt Juliane House (1997) die interkulturelle Kompetenz der kommunikativen gleich und begründet das damit, dass die unterschiedlichen Definitionen der beiden Kompetenzen die gleichen Fähigkeiten angeben würden. Des Weiteren kritisiert sie den interkulturellen Ansatz in Bezug auf das Herunterspielen der sprachlichen Bestandteile und die Hervorhebung der affektiv- verhaltensorientierten Ziele des Fremdsprachenunterrichts.

„Lehr- und Lernziele im Fremdsprachenunterricht werden traditionell in drei Bestandteile gegliedert: Wissen, Haltungen/Einstellungen und sprachpraktische Fertigkeiten, d.h. in eine kognitive, eine affektive und eine skillspezifische Domäne. Die ,mainstream'-Konzeption interkultureller Kompetenz im FU ist nun, so möchte ich argumentieren, einseitig auf die affektive Domäne kapriziert (Empathie, Verstehen, Toleranz und so weiter) zum Nachteil und Schaden der anderen beiden Domänen“" (House 1996, S. 3). Dieser Meinung ist nur dann zuzustimmen, wenn die interkulturelle Kompetenz als Gegenpol zum Konzept der kommunikativen Kompetenz betrachtet wird. Der interkulturelle Ansatz kann aber auch als Erweiterung der kommunikativen Kompetenz verstanden werden, wenn er nicht nur neue Inhalte einbringt, sondern auch die alten Inhalte beibehält. Der interkulturelle Ansatz setzt sicherlich andere Schwerpunkte im Hinblick auf die Zielsetzung, verzichtet aber nicht auf die im Laufe seiner Entstehung ausgearbeiteten Inhalte, so wie zum Beispiel die Arbeit an der Grammatik, Wortschatz, Phonetik oder auf die in den letzten Jahrzehnten definierten Ziele in Bezug auf den Diskurs, den Kontext sowie die korrekte Kommunikation. Mit dieser Ansicht stimmt die Autorin mit Vollmer überein, der behauptet, dass „,der Qualifikator ,interkulturell' (wenngleich stark modisch geprägt) keineswegs überflüssig ist oder gar ein ,Etikettenschwindel'. Andererseits stellt er kein wirklich neues Paradigma in der Sprachforschung dar“" (Vollmer 1993, S. 108).

Lag früher der Schwerpunkt auf der Kommunikation selbst als Prozess einer zwischenmenschlichen Interaktion mit dem Ziel einer Verständigung, wird jetzt der kulturelle Hintergrund oder der soziokulturelle Kontext der Sprechenden als ebenso wichtig betrachtet. Während bei dem kommunikativen Ansatz der kulturelle Kontext als eine relativ konstante Erscheinung betrachtet wird, wird bei dem interkulturellen Ansatz eine Kultur nicht als eine einheitliche Erscheinung angesehen, und die Gesprächspartner werden als Vertreter mehrerer kultureller Kreise oder kultureller Bezugssysteme verstanden. 
„Als kulturelle Bezugssysteme werden dann nicht mehr konkrete Gruppen oder Gemeinschaften von Menschen bezeichnet, sondern abstrakte Bezugsgruppen, mit denen sich Menschen identifizieren bzw. denen sie von anderen zugeordnet werden“ (Flechsig 2001, S. 5).

Das bedeutet für einen Gesprächspartner, dass es keinen konstanten oder vorprogrammierbaren soziokulturellen Kontext gibt, genauso wie es keinen generalisierten native speaker, dessen Normen und Werte als ideales Beispiel für das Wertesystem der jeweiligen Kultur dienen können, gibt. Die gleiche Ausdifferenzierung gilt für den Lernenden selbst, der als Individuum sowohl kulturell als auch kommunikativ keine „tabula rasa“ ist und die Einstellungen von unterschiedlichen kulturellen Kreisen, denen er sich zugehörig fühlt, mit in das Gespräch einbringt.

Zusammenfassend lässt sich sagen, dass die beiden Ansätze (sowohl kommunikativer als auch interkultureller Natur) als Ziel die Verständigung zwischen Gesprächspartnern mit unterschiedlichen Muttersprachen haben. Der kommunikative Ansatz betont den Prozess der Kommunikation, der interkulturelle setzt seine Prioritäten im soziokulturellen Kontext.

Die Meinung von House (1996), dass die interkulturelle Kompetenz „einseitig auf die affektive Domäne kapriziert (...) zum Nachteil und Schaden der anderen beiden Domänen“ (House 1997, S. 3) und damit sind die kognitive und die skillspezifische Domänen gemeint, kann nicht zugestimmt werden, weil m.E. das interkulturelle Lernen nicht nur auf die Veränderung der Einstellungen und Haltungen gerichtet ist, sondern es zielt vielmehr auch auf die vierte Domäne, auf die Veränderung des Bewusstseins (so in den Arbeiten von Alvaro Fantini 2000).

„Inhaltlich geht es um das Erkennen und die Aneignung einer fremden Sprache, nicht nur als System, sondern als Ausdruck eines fremden Denkens und einer fremden Lebenspraxis. Dabei ist der Prozess des kontrastiven Lernens, des Vergleichs, der Herausarbeitung der eigenen kulturell vermittelten Bedeutungen und (Vor-)Urteile und deren Vermittlung mit den neuen Positionen und Bewertungen gleichermaßen wichtig“ (Vollmer 1993, S. 109).

Die Bedeutung der kognitiven Komponente wird besonders bei der Aneignung des Wissens deutlich.. Der Kreis der ,wissenswerten“ Informationen wächst über die sprachlichen und landeskundlichen Kenntnisse durch Aneignung des Wissens über Wahrnehmung und Verhalten in den interkulturell geprägten Situationen - sowohl allgemeines Wissen über solche Erscheinungen wie Kultur und kulturelle Orientierungen als auch kulturelle Stereotype und Missverständnisse - hinaus. Was den praktischen Aspekt des Spracherlernens anbelangt, 
hat er nach wie vor eine große Bedeutung, welche kaum zu verleugnen ist. Niemand wird die Tatsache bezweifeln, dass ohne sprachliche Fähigkeiten und Fertigkeiten eine Kommunikation in einer fremden Sprache nicht möglich ist. Die interkulturelle Kompetenz erfordert aber noch zusätzliche, im interkulturellen Bereich liegende Fertigkeiten, die m.E. am schwersten zu erreichen sind.

Das oben angeführte Zitat von Vollmer weist außerdem auf die große Rolle unterschiedlicher Methoden beim Erwerb der kommunikativen und interkulturellen Kompetenzen hin. Die kontrastive Methode oder der Vergleich der fremden und der eigenen sprachlichen und kulturellen Einheiten bekommen beim interkulturellen Ansatz eine entscheidende Rolle. Was bei der kontrastiven Methode zu schätzen ist, ist der besondere Wert der eignen kulturellen Einstellungen. Die eigene Position des Sprachlernenden wird auch beim Erwerb von „kommunikativer Kompetenz“ beachtet, und zwar mit folgender Zielstellung: der Lernende muss imstande sein, die eigentliche Position mit Hilfe einer fremden Sprache ausdrücken zu können. D.h. es wird akzeptiert, dass der Lernende eine eigene Position besitzt, die er gegenüber einer anderen fremden Position zum Ausdruck bringt. Bei dem interkulturellen Ansatz wird erstens mehr Wert auf die kulturellen Wurzeln dieser Position und die Ausdifferenzierungen dieser Wurzeln gelegt. Der zweite wesentliche Unterschied, was die eigene Position des Sprachlernenden angeht, ist die Tatsache, dass diese Position nicht konstant betrachtet wird. In dem Moment, wo der Mensch durch die fremde Sprache mit einer fremden Position konfrontiert wird, begibt sich seine eigene (kulturell geprägte) Position in einen Veränderungsprozess. Die angewandte kontrastive Methode fordert von dem Lernenden, die eigenen Positionen in Frage zu stellen und dann durch den Vergleich mit den fremden Positionen etwas dazuzulernen. Der Kommunikationsprozess selbst wird dann wie eine Art Aushandlungsprozess verstanden, infolge dessen sowohl kulturell geprägte als auch durch eigene Erfahrungen erworbene Einstellungen und Werte des Lernenden (obwohl die beiden Arten kaum voneinander zu trennen sind) ständig weiterentwickelt werden.

„In der konkreten Begegnungssituation entwickelt sich (...) ein Interaktions- und Aushandlungsprozess, der sich nicht allein aus den beteiligten Kulturen und ihren Repräsentationen erklären lässt. Vielmehr entsteht hier eine Zusammenarbeit, ein synergetisches Zusammenspiel, das sich auf die gemeinsame Erfüllung der kommunikativen Aufgaben richten und die zu Regeln; Schlüsseln und Prozessen eigener (lokaler) Art führen kann“ (Vollmer 1993, S. 110).

Dies bringt einen weiteren Aspekt der interkulturellen Kompetenz zum Ausdruck. Im Un- 
terschied zum kommunikativen Ansatz legt der interkulturelle Ansatz einen größeren Wert auf die erzieherische Bedeutung eines solchen Unterrichts. Die Aneignung einer Kultur wird, wie aus der Psychologie bekannt, „Sozialisation“ genannt. Dieses Axiom gilt bezüglich der ersten Kultur; was geschieht aber, wenn der Lernende eine fremde Kultur mittels einer erlernten Fremdsprache sich anzueignen versucht? Der interkulturelle Ansatz behauptet, dass eine Art zweite Sozialisation passiert, und zwar die sogenannte Akkulturation, wenn der Lernende seine eigenen Einstellungen oder Werte der zu erlernenden Kultur anzupassen versucht. Obwohl diese besondere Akzeptanz der erzieherischen Rolle der fremden Kultur oft kritisiert wird, entwickeln sich zurzeit einige Theorien, wie zum Beispiel die Theorie der sekundären „Sprecherpersönlichkeit" ${ }^{\text {“8 }}$ in der russischen Didaktik (Galskaja 2004, Haleeva 1995).

Zum Schluss der Diskussion darüber, dass kommunikative und interkulturelle Kompetenz als Ziele des Fremdsprachenunterricht nicht gleichgesetzt werden können, möchte ich die im Einzelnen kritisierten Schwächen des Konzepts „kommunikative Kompetenz“ anführen, die gleichzeitig auf neue konzeptuelle Inhalte des interkulturellen Ansatzes hinweisen.

- Das Konzept „kommunikative Kompetenz“ verleitet dazu, sich an den Normen eines generalisierten native speaker zu orientieren.

- Es wird nicht genügend differenziert zwischen Alltagskommunikation und institutionell geprägten Handlungsrollen.

- Es wird versäumt, den Erwerb von kommunikativer Kompetenz als einen Prozess der Anpassung und Akkulturation zu sehen.

- Es wird der Eigendynamik sozialer Interaktion nicht gerecht und bereitet nicht angemessen darauf vor.

- Es schließt den sprachlich-kulturellen Hintergrund des Lerners nicht genügend mit ein.

- Es ist demnach nicht wirklich interkulturell angelegt (vgl. Baxter 1983, Vollmer 1993).

Der interkulturelle Ansatz ist in der Fremdsprachendidaktik eine relativ neue Erscheinung, die sich unter dem Einfluss von unterschiedlichen Wissenszweigen entwickelt hat. Dies führt zu einem gewissen Durcheinander der in der Terminologie verwendeten Begriffe, die

8 Unter einer „Sprecherpersönlichkeit“ wird eine Person verstanden, die fähig ist, in der Situation einer authentischen Kommunikation mit Vertretern einer Kultur Sprechhandlungen auszuführen. Durch die Identifizierung mit dem fremden kulturellen Hintergrundwissen und dem Auslöschen der Fremdheit im Bewusstsein der Sprachlernenden ist es möglich, die neu zu erlernende Kultur als Zweitkultur zu betrachten. Dabei bekommt die Persönlichkeit eines Lernenden die Charakterzüge einer zweiten Sprecherpersönlichkeit. 
auf ihre Herkunftswissenszweige verweisen. Der interkulturelle Ansatz hat aber seine besonderen Schwerpunkte und Ideen, deshalb könnte er durchaus als ein neuer Weg in der Didaktik des Fremdsprachenunterrichts sein. Die interkulturelle Kompetenz als oberstes Ziel dieses Ansatzes kann ihrerseits nicht mit der kommunikativen Kompetenz gleichgesetzt werden, weil die beiden Kompetenzen unterschiedliche Schwerpunkte haben.

\section{Theoretische Konzepte zur interkulturellen Kompetenz}

Im nächsten Kapitel sollen einige in der wissenschaftlichen Literatur existierende Theorien zur „,interkulturellen Kompetenz“ vorgestellt werden. Begonnen wird mit der im Bereich der sozialen Arbeit entwickelten modernen Theorie von Fischer (2007), dann wird auf die grundlegenden Konzepte von Fantini (2000), Byram (1997) und Brislin und Yoshida (1994) eingegangen, und schließlich wird mit dem Bericht über die Arbeit von Deardorff eine Zusammenfassung von unterschiedlichen Theorien zur interkulturellen Kompetenz gegeben. Am Ende des Kapitels wird ein Puzzle-Modell der interkulturellen Kompetenz, welches dieser Dissertation zugrunde liegt, vorgestellt. Es ist zu erwähnen, dass alle Theorien nicht auf Umfeld und Bedingungen des Fremdsprachenunterrichts gerichtet sind, sondern meistenteils auf den Bereich sozialer Arbeit oder interkultureller Unternehmen.

„Wenn man versucht, sich einen Überblick über die Diskussion zum Thema interkulturelle Kompetenz zu verschaffen (...), so kann einen die Fülle des Materials ratlos machen“ (Auernheimer 2002, S. 183). In diesem Kapitel wird nicht das Ziel verfolgt, einen vollständigen Überblick über alle existierenden Theorien zu geben, da erstens die Zahl dieser Theorien enorm groß ist und zweitens sich viele von ihnen auf die Auflistung bestimmter Fähigkeiten oder Teilkompetenzen, die den Menschen erlauben in der interkulturellen Situationen kompetent zu handeln, reduzieren. Diese Theorien werden ausgeschlossen, weil es ihnen an zwei wichtigen Komponenten fehlt: sie gehen nicht auf die kognitive Seite der Kompetenz ein, d.h. es wird wenig über die Notwendigkeit der Vermittlung des Fachwissens gesprochen. Zweitens wird kaum oder fast nie die Tatsache erwähnt, dass für die interkulturelle Kompetenz bestimmte Haltung und interkulturelles Bewusstsein notwendig ist. In diesem Kapitel werden Theorien diskutiert, die versuchen, unterschiedliche Dimensionen der interkulturellen Kompetenz in ein Ganzes einzubinden, auch unter Beachtung der Rahmenbedingungen, in denen diese Kompetenz gebraucht wird. 
Eine der modernsten Theorie der interkulturellen Kompetenz im deutschsprachigen Raum ist die der Erziehungswissenschaftlerin Veronika Fischer, die das Anwendungsgebiet ihrer Theorie in der sozialen Arbeit mit Migrantenfamilien und Kindern mit Migrationshintergrund sieht. Da das angestrebte Arbeitsfeld festgelegt ist, beschränkt sich die Verfasserin nicht auf die Aufzählung von Teilkompetenzen, sondern bildet ein System aus. So unterscheidet sie drei Ebenen, in die sich die interkulturelle Kompetenz aufschlüsseln lässt, und zwar eine globale, eine gesellschaftliche und eine institutionelle Ebene. Interkulturell kompetent auf der globalen Ebene zu handeln heißt, die unterschiedlichen weltweit verlaufenden Prozesse der Globalisierung, die Durchdringung der nationalen Elemente in einem weltweiten Kontext, sei es in den ökonomischen, kulturellen, politischen oder sozialen Bereichen, bewusst wahrzunehmen und damit umgehen zu können. Eine globale Ebene schließt eine gesellschaftliche ein, die ihrerseits eine institutionelle beinhaltet. Unter der gesellschaftlichen Ebene wird eine multikulturelle Gesellschaft verstanden, in der die ökonomischen, sozialen und kulturellen Strukturen das Leben jedes einzelnen ihrer Mitglieder beeinflussen. Die institutionelle Ebene ist der Kontext der „,interkulturellen Überschneidungssituation ${ }^{\star 9}$ selbst, bei der das hauptberufliche Personal einer Einrichtung auf den Klienten mit Migrationshintergrund trifft.

9 Interkulturelle Überschneidungssituation wird eine Situation genannt, bei der Vertreter unterschiedlicher Kulturen kommunizieren, wobei sich ihre kulturelle Orientierungssysteme überlappen und es entsteht dabei ein zwischenkultureller Raum. (Siehe Abbildung 6) 


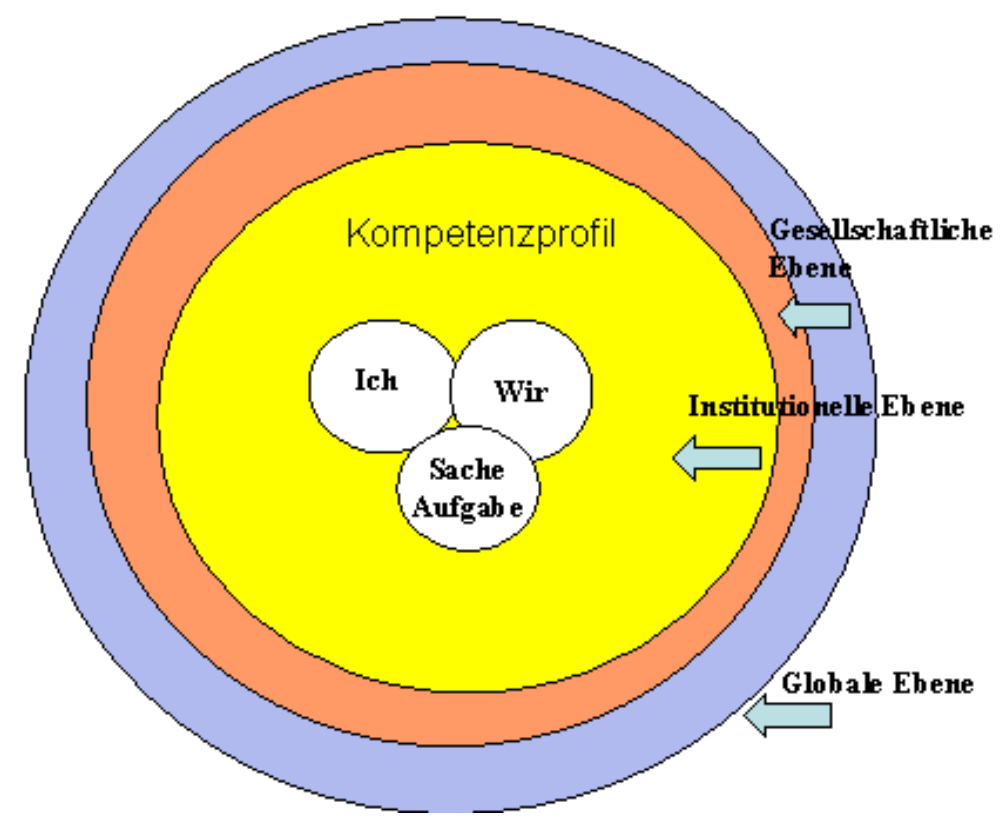

(Quelle: Fischer o. J., S. 38)

Die Theorie von Fischer teilt alle Kompetenzen in drei Gruppen, wobei es aber nicht nur um Fähigkeiten geht, sondern auch um Einstellungen und Haltungen. Die interkulturellen Kompetenzen präsentieren sich in der Ich-Ebene, der Wir-Ebene und der Sach-Ebene. Die Ich-Ebene wird von der Autorin „selbstreflexive Kompetenz“ genannt, dabei geht es um Anerkennung und Akzeptanz der eigenen Fremdheitsgefühle. „Erst in der Auseinandersetzung mit den unbewussten Anteilen unserer Differenzerfahrung lernen wir die affektiven Reaktionsformen von den kognitiven Bewältigungs- und Rechtfertigungsmustern zu unterscheiden“ (Fischer 2005, S. 41). Durch das Einnehmen einer Beobachterrolle sollte zum Beispiel ein Sozialarbeiter zu der eigenen alltäglichen Haltung, den eigenen Werten, Normen und Gefühlen einen Abstand schaffen, was ihm erlaubt, ethnozentrische Beurteilungen zu vermeiden.

Die Wir-Ebene schließt ein Bündel sozialer Kompetenzen ein, die eine Person befähigen, eine andersartige Perspektive des fremdkulturellen Partners zu erkennen und sich ihm gegenüber sensibel zu verhalten. Dabei sind solche rein sozialen Kompetenzen wie Empathie, Ambiguitätstoleranz, Akzeptanz und Fähigkeiten zur Konfliktaustragung von besonderer Bedeutung.

„Interkulturelle Professionalität zeigt sich daher im Einverständnis, nie alles vom anderen wissen zu können, in dem Bemühen, sich der Sicht des anderen annähern zu wollen, in der Ablehnung kolonialer Vereinbarung des Klienten und der Akzeptanz des anderen als Ex- 
perten seiner Lebenswelt“" (Fischer 2005, S. 43).

Als eine der Komponenten der Wir-Ebene wird von Fischer die Sprachkompetenz oder die kommunikative Kompetenz genannt. Damit meint sie weniger die Beherrschung einer Fremdsprache, denn das Erlernen einer Migrationssprache hält sie für empfehlenswert, sieht aber Schwierigkeiten darin, dass Sozialarbeiter normalerweise mit den Vertretern mehrer ethnischer Gruppen zu tun haben, sodass das Erlernen aller Sprachen der Klienten nicht möglich ist. Vielmehr bezieht sie die kommunikative Kompetenz auf die Beherrschung der Fähigkeit, ein Gespräch so zu führen, dass die Normen eines Sprechaktes aus der kulturellen Sicht des Klienten möglichst wenig verletzt und die paraverbalen und nonverbalen Komponenten einer Kommunikation beachtet werden.

Als Sach-Ebene bezeichnet Fischer sowohl das Wissen über die unterschiedlichen Aspekte der Migration, Zuwanderung und Multikulturalität als auch spezifisches Wissen über kulturelle Hintergründe der Migranten, die aus unterschiedlichen Ländern stammen. Dabei warnt sie davor, solche Informationen zu stereotyp aufzunehmen und sie einfach in Schubladen nach den Herkunftsländern zu sortieren, da sowohl die Sozialisationsprozesse als auch die Migrationsprozesse sehr komplex sind.

Die Theorie scheint grob die wichtigsten Anteile der kommunikativen Kompetenz zu enthalten und auf einen bestimmten Personenkreis ausgerichtet zu sein. Das kann vorteilhaft sein, kann aber auch einen Nachteil haben, der darin besteht, dass die Theorie einen ziemlich einseitigen Charakter hat, und zwar weil sie alles nur aus der Sicht der Mitarbeiter der sozialen Strukturen betrachtet. Für die erfolgreiche interkulturelle Kommunikation ist aber m.E. eine zweiseitige Beziehung vonnöten. Für ein erfolgreiches Zusammenleben in einer multikulturellen Gesellschaft muss die interkulturelle Kompetenz deshalb auch von den Migranten gefordert werden.

\section{Das Modell interkultureller Kompetenz von Alvino Fantini (2000)}

Die Theorie von Alvino Fantini (2000) nimmt eine Zwischenstellung ein, die sowohl die Interessen der sogenannten ,interculturalists“"10 als auch die Interessen der Sprachlehrer beachtet. Das heißt, dass der Autor ein Modell entwickelt hat, das seine Anwendung sowohl in der multikulturellen Gesellschaft als auch im Fremdsprachenunterricht findet.

Fantini behauptet, interkulturelle Kompetenz bestehe aus fünf Dimensionen, und zwar aus

${ }^{10}$ Als ,interculturalists“ bezeichnet Fantini diejenigen Fachleute, die im Bereich cultural studies ihren Beitrag leisten. 
Kenntnissen (knowledge), Fähigkeiten (skills), Einstellungen oder Haltungen (attitudes) und Bewusstsein (awareness). Außerdem vertritt er die Meinung, dass die Fähigkeiten in einer Fremdsprache eine unabdingbare Bedingung für die interkulturelle Kompetenz darstellen. Eine besondere Aufmerksamkeit schenkt er zwei Positionen in seiner Theorie. Zum einen versteht er das Bewusstsein ${ }^{11}$ nicht nur als eine selbstständige Dimension der Kompetenz, sondern als eine Dimension der erfolgreichen Interaktion, die eine zentrale Stelle in seinem Modell einnimmt. Das Bewusstsein hat, so Fantini, immer mit „Selbst“ zu tun, und zwar das Verhältnis des „Selbst“ zu ,jemandem“ oder zu „etwas anderem, Fremdem“. Er bezeichnet es als eine Art Selbstreflexion, die eine Erkundigung, eine Erprobung und ein Erlebnis oder eine Erfahrung voraussetzt. Ein Bewusstsein kann nur infolge einer Selbstanalyse und Selbstuntersuchung zustande kommen.

„Awareness is difficult to reverse; that is, once one becomes aware, it is difficult to return to a state of unawareness (...). Awareness leads to deeper cognition, skills, and attitudes just as it also enhances by their development. It is pivotal to cross-cultural entry by members of other cultures on their terms“" (Fantini 2000, S. 29).

Das Bewusstsein hilft, sich selbst in einer sozialen Situation kritisch zu beobachten und eigenes Verhalten in der realen Situation zu steuern.

Abbildung 2: Modell von Fantini (2000)

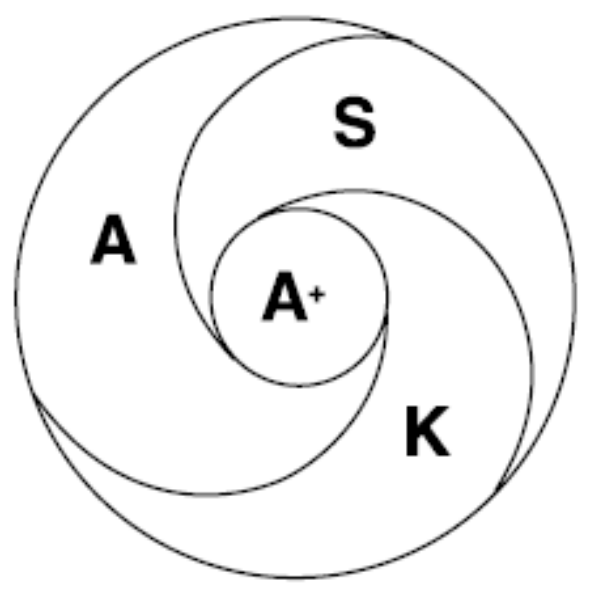

A - attitudes (Einstellungen oder Haltungen)

$\mathbf{S}$ - skills (Fähigkeiten)

K - knowledge (Kenntnisse)

A+ - awareness (Bewusstsein)

Quelle: Fantini 2000, S. 28

Der zweite wichtige Aspekt in den Arbeiten von Fantini ist seine Erkenntnis, dass interkul-

11 Um den Ursprung des Begriffes zu finden, verweist der Autor auf die Arbeiten von Paulo Freire, der den Begriff „,critical consciousness“ oder „awareness“ verwendet hat. Dieser Begriff fand später eine internationale Anerkennung. 
turelle Kompetenz ohne Fremdsprachenbeherrschung gar nicht möglich ist. „A monolingual who has never grappled with a foreign communication system may develop many intercultural talents but will be excluded from the arising from the struggle to communicate in alternative ways and the differing conceptualizations encoded in other language systems“" (Fantini 2000, S. 29).

\section{Die Theore von Michael Byram (1997)}

Die Theorie der interkulturellen kommunikativen Kompetenz des britischen Kulturforschers Michael Byram (1997) stellt eine Essenz von unterschiedlichen soziolinguistischen, anthropologischen und psychologischen Theorien dar. Er hat seit den 70er Jahren untersucht, in welchem Verhältnis das Erlernen einer Fremdsprache und das Erlernen einer Kultur zueinander stehen oder welche Folge dieser gemeinsame Unterricht für die menschliche Persönlichkeitsentwicklung hat. Diese Forschung hat ihren Niederschlag in seinen früheren Theorien gefunden (so z.B. im Model for Language und Cultural Teaching 1989). 1997 veröffentlichte er ein Modell, welches eine breite Anerkennung erfuhr und im Prinzip als Grundmodell für einige weitere Arbeiten dient. Das Modell ist an der Fremdsprachendidaktik orientiert, dabei ist die interkulturelle Kompetenz in einen Kreis von weiteren Kompetenzen - linguistischen, soziolinguistischen und Diskurskompetenzen - verflochten.

Sein Modell besteht aus fünf Teilen:

1) savoir - Wissen

2) savoir être - Einstellungen

3) savoir comprendre - Fähigkeit, zu interpretieren und Beziehungen herzustellen

4) savoir apprendre/faire - Lern- und Entdeckungsfähigkeit

5) savoir s'engager - kulturelles Bewusstsein (vgl. Byram 1997)

Ebenso wie Fantini hebt Byram Wissens- Einstellungs- und Bewusstseinskomponenten hervor. Was aber auffällt, sind zwei Gruppen von unterschiedlichen Fähigkeiten. „Savoir comprendre“ interpretiert er als Fähigkeit zur Interpretation von Texten, Filmen, Bildern und anderen Informationsträgern aus unterschiedlichen kulturellen und historischen Kontexten. Der Lernende soll auch fähig sein, das Ergebnis dieser Interpretation in Verbindung mit eigenen kulturellen Erfahrungen zu bringen und dadurch neue Seiten seines Weltbildes zu erarbeiten. Die zweite Gruppe der Fähigkeiten, savoir apprendre/faire genannt, bezieht sich auf das selbstständige Aufsuchen dessen, was in irgendeiner Weise anders ist, und auf die Fähigkeit, damit umzugehen. 
Das Modell von Byram ist ein Beispiel für den hermeneutischen Ansatz im Fremdsprachenunterricht, der auf der Grundüberzeugung basiert, dass ein Mensch Vertreter unterschiedlicher kultureller Schichten ist und dass seine Persönlichkeit infolge unterschiedlicher kultureller Erfahrungen geformt wurde. Das Erlernen einer Fremdsprache soll infolge der Konfrontation mit der neuen Kultur zur Persönlichkeitsentwicklung des Lernenden beitragen, zu einer Art zweiter Sozialisation. Die Frage, welche Sprache als Medium für den Kulturunterricht benutzt werden soll, beantwortet der Autor durch die Feststellung, dass beide Sprachen, sowohl die Mutter- als auch die zu erlernende Fremdsprache, aus dem Unterricht Gewinn ziehen könnten. (vgl. Byram 1989, 1997)

Abbildung 3: Modell von Byram (1997)

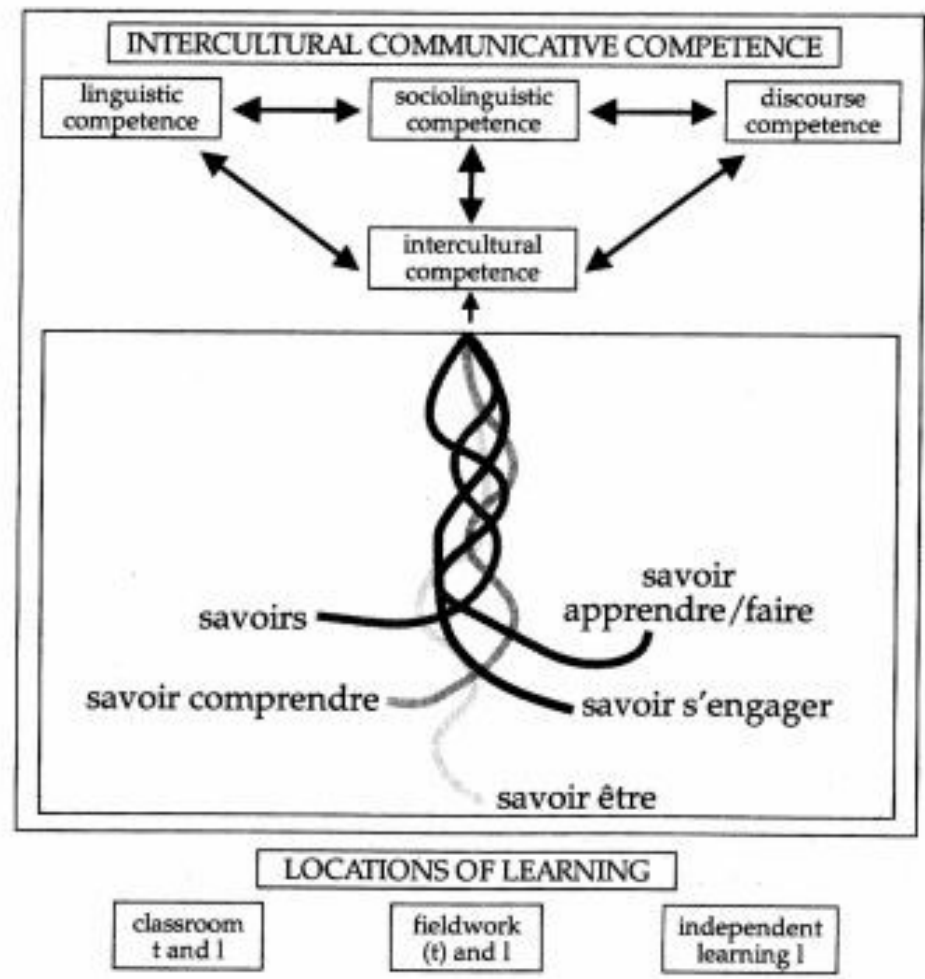

Quelle: Byram 1997, S. 34

Das Modell des interkulturellen kommunikativen Trainings von Richard Brislin (1994)

Der amerikanische Psychologe Richard Brislin, der sich mit der Frage beschäftigte, welche Auswirkung die Kultur auf das Verhalten eines Menschen hat, entwickelte in Kooperation mit Tokomo Yoshida auch ein Programm für ein interkulturelles Training. Die Autoren gliedern die Trainingsmaßnahmen so, dass bestimmte Teile der angestrebten Kompetenz zu 
sehen sind: Awareness (Bewusstsein), Knowledge (Kenntnisse), Emotional Challenges (emotionale Komponente) und Behavior (Verhalten) (vgl. Brislin und Yoshida 1994).

Das Unterrichtsmodell von Valentina Furmanova (1993)

Als letztes soll ein Unterrichtsmodell von Furmanova erwähnt werden, dessen Ziel die Entwicklung der interkulturellen Kompetenz ist. Sie gliedert den Unterrichtsprozess einer Fremdsprache in zwei Modi: kognitive Steuerung der Wissensaneignung über kulturelle Realität (культурный фон) und die Entwicklung des kulturellen Modus des Verhaltens (культурный модус поведения). Zur Aufgabe des zweiten Modus gehört nicht nur die Entwicklung der kommunikativen Technik, sondern auch die Fähigkeit, sich auf seinen Gesprächspartner einzustimmen, um ein gegenseitiges Verständnis zu erreichen (vgl. Furmanova 1993).

Zusammenfassend lässt sich Folgendes sagen: Obwohl die angeführten Theorien aus unterschiedlichen Wissensgebieten stammen, was in ihrer Struktur und in ihrem Anwendungsbereich zu sehen ist, sind sie alle durch einige gemeinsame Merkmale gekennzeichnet: Interkulturelle Kompetenz stellt kein einheitliches Konzept dar und kann in Teilbereiche gegliedert werden.

1. Alle Wissenschaftler weisen auf mindestens zwei unabdingbare Bestandteile der Kompetenz hin, und zwar „Wissen“ und „Fähigkeiten“.

2. Sehr oft werden Bestandteile wie „Einstellungen oder Haltungen“ und/oder „Bewusstsein“" erwähnt.

3. Die Beziehung zu sich selbst und zum Fremden (zu sich und dem Partner, zu eigener und fremder Gesprächsposition) scheint in allen Theorien einen wichtigen Platz einzunehmen.

4. Das Konzept der interkulturellen Kompetenz hat eine dynamische Struktur, und die Entwicklung der einzelnen Bestandteile fördert die Entwicklung der anderen.

5. Die Entwicklung der interkulturellen Kompetenz hat eine unmittelbare Verbindung zu der fremden Sprache, obwohl die Meinungen der Wissenschaftler hier auseinandergehen in Bezug darauf, ob Sprachbeherrschung oder Kommunikation in der fremden Sprache ein notwendiger Teil der interkulturellen Kompetenz ist.

Diese fünf Säulen werden die Grundlage für das in dieser Arbeit vorgelegte Puzzle-Modell (vgl. Kapitel 4) sein. Zuvor sollen aber noch die Ergebnisse einer Studie vorgestellt wer- 
den.

Wie bereits erwähnt, ist die interkulturelle Kompetenz ein aktuelles und populäres Thema, was zu unterschiedlichen Theorien, Beschreibungen, Modellen und Definitionen geführt hat. Aufgrund der Vielfältigkeit der dazu existierenden Ansichten hat sich die Bertelsmann Stiftung als Aufgabe gestellt, zum einen den Diskussionstand zu diesem Konzept zu erforschen, zum anderen einen Überblick zu verschaffen und letztendlich zu versuchen, ein gemeinsames interdisziplinäres Modell der interkulturellen Kompetenz zu liefern. Im Auftrag der Bertelsmann Stiftung führte die Wissenschaftlerin Darla Deardorff 2004-2005 eine Untersuchung durch, im Verlaufe derer die führenden US-amerikanischen Experten (darunter auch Bennett und Byram, deren Modelle oben dargestellt wurden) zum Thema befragt wurden. ${ }^{12}$ Aus den vorgeschlagenen sieben Definitionen des Begriffs haben die Experten am häufigsten die folgende ausgewählt:

„Interkulturelle Kompetenz ist die Fähigkeit, effektiv und angemessen in interkulturellen Situationen zu kommunizieren, auf Grundlage eigenen interkulturellen Wissens, Fähigkeiten und Einstellungen“ (Deardorff o.J., S. 14).

In dieser Definition sind die drei bereits diskutierten Bestandteile der Kompetenz enthalten. In der weiteren Untersuchung sollte herausgefunden werden, was interkulturelles Wissen, interkulturelle Fähigkeiten und Einstellungen ausmacht. Dabei wurden die 22 wichtigsten Teilelemente herauskristallisiert, welche später in ein Pyramidenmodell der interkulturellen Kompetenz eingearbeitet wurden. Das Modell enthält vier Teile: Wissen und Fähigkeiten, Einstellungen und Haltungen, interne Wirkung und externe Wirkung.

In diesem Gesamtmodell sind deutlich die Züge der oben vorgestellten Theorien zu erkennen. Was einige Wissenschaftler (wie Fantini oder Byram) als vereinzelte Bestandteile der Kompetenz betrachten, wird hier in einem Cluster zusammengefasst. Die beiden Komponenten Wissen und Fähigkeiten werden in zwei ,separate, aber einander ergänzende und komplementäre Untergruppen“ (Deardorff o.J., S. 18) aufgeteilt. Zur Komponente Wissen

12 Diese Studie wurde in zwei Schritten durchgeführt. Im ersten Schritt wurden die Fragebögen unter den für die Internationalisierungsstrategien US-amerikanischer Hochschulen verantwortlichen Managern verteilt. Neben der Beantwortung von einigen offenen und geschlossenen Fragen darüber, wie deren jeweilige Institution interkulturelle Kompetenz als Lernziel der Studierenden fördert, sollten die Manager Namen von national und international anerkannten Experten bestimmen, die in dem zweiten Schritt der Studie teilgenommen haben. Insgesamt 23 von 37 der am häufigsten genannten Experten haben ihre Zusage für die Teilnahme am zweiten Schritt erklärt. Hier wurde als Untersuchungsmethode das dreistufige Delphi-Verfahren herangezogen. In der ersten Stufe sollten die Experten die zwei Fragen beantworten (Was macht interkulturelle Kompetenz aus? Wie kann man interkulturelle Kompetenz messen?), deren Analyse zur Zusammenstellung der Elemente dieser Kompetenz führte. Diese wurden in der zweiten Stufe zur Bewertung an die Experten geleitet, die noch weitere Elemente hinzufügen konnten. Die Ergebnisse wurden in ein Modell gefasst, das in der dritten Runde den Experten zur Bewertung vorgelegt wurde. 
wird nicht nur kulturspezifisches Wissen, sondern auch Selbstreflexion als Ausgangspunkt zum Verständnis der fremden Kulturen gezählt. Zum Wissen gehört auch kulturelles Bewusstsein; das Fantini in seinem Modell als selbstständige Überkomponente bezeichnet hat. Die in diesem Cluster präsentierten Fähigkeiten (Zuhören, Beobachten, Interpretieren, Analysieren usw.) liegen im kognitiven Bereich und verweisen auf „savoir comprendre“ von Byram. Das Ziel dieser Fähigkeiten sollte die selbstständige Weiterentwicklung der interkulturellen Kompetenz durch Wissenserwerb und -evolution sein. Dabei werden die beiden Komponenten unter dem Titel Handlungskompetenz vereint.

Abbildung 4: Modell von Deardorff (o.J.)

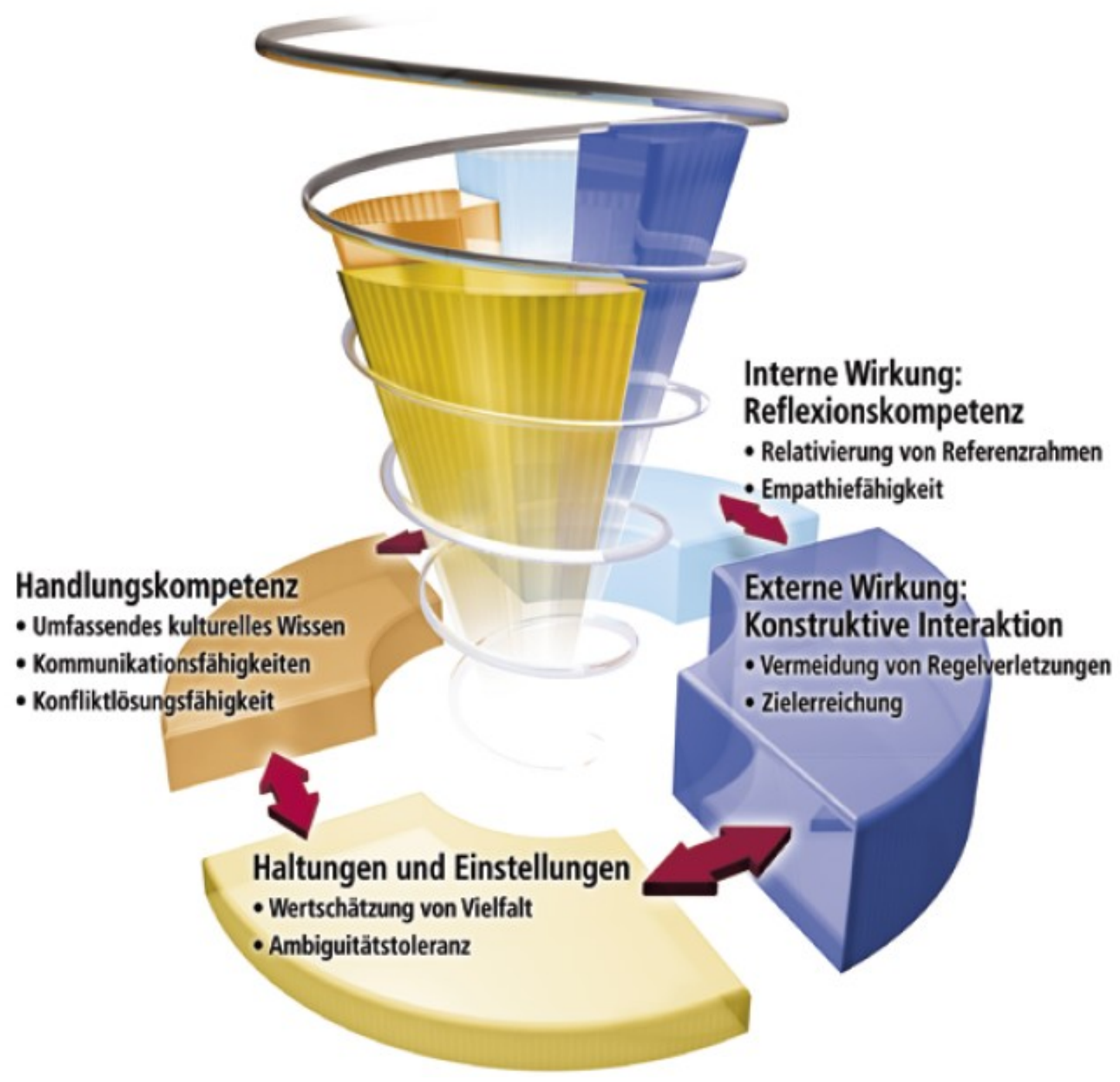

(Quelle: Deardorff o.J., S. 7)

Das zweite Cluster bilden die Haltungen und Einstellungen. Deardoff und mit ihr auch die meisten befragten Experten unterstreichen die grundlegende Bedeutung dieser Komponen- 
te. In den Theorien zur interkulturellen Kompetenz, die sich gerade auf die Aufzählung der Eigenschaften und Fähigkeiten begrenzen, sind meistens die Elemente dieses Clusters vertreten, so wie Ambiguitätstoleranz, Offenheit, Respekt usw.

Bei den Definitionen fiel den Autoren die sich wiederholende Gegenüberstellung von „Ich und das andere“ auf. Deshalb haben sie ein drittes Cluster mit zwei Kategorien gebildet: erwünschte interne Wirkung der interkulturellen Kompetenz (Perspektivenwechsel, Flexibilität, Empathie) und erwünschte externe Wirkungen (angemessenes Verhalten und Fähigkeit, Ziele zu erreichen). In diesem dritten Cluster sind der Einfluss der Sozialpädagogen zu erkennen: genauso teilt Fischer in ihrer Theorie die Kompetenzen in Ich-Ebene (intern) und Wir- und Sach-Ebene (extern) ein.

Deardorff versucht in ihrem Modell die in unterschiedlichen Gebieten entstandenen Theorien zur interkulturellen Kompetenz zusammenzufassen, darin liegt ihr Vorteil und auch ihr Nachteil. Obwohl diese Theorie alle interessanten Seiten am umfangreichsten beleuchtet (sie benennt die größte Zahl benötigter Fähigkeiten und Einstellungen bzw. sie verbindet in einem Modell sowohl Bestandteile wie auch den zweiseitigen Charakter der Kompetenz), erscheint sie teils zweideutig und nicht ausgewogen. Bei Aufzählung der Fähigkeiten treffen wir nur auf kognitive und kaum soziale wie Empathie oder Anpassungsfähigkeit. Es kann zugestimmt werden, dass sie als erwünschte interne Wirkung angesehen werden, sie gehören aber auch zu den notwendigen Fähigkeiten der Kompetenz.

Zusammenfassend lässt sich sagen, dass die oben dargestellten Theorien einen Überblick über die in den unterschiedlichen Wissenschaftszweigen existierenden Theorien zur interkulturellen Kompetenz geben. Es lässt sich feststellen, dass zwei grundsätzliche Richtungen existieren: Nach einigen Theorien wird die interkulturelle Kompetenz in ihre Bestandteile gegliedert, wie Wissen, Fähigkeiten, Bewusstsein, emotionale Komponente und einige andere. Die Theorien der zweiten Richtung unterteilen die notwendigen Kompetenzen bezüglich ihres Verhältnisses des „Ich“ zum ,anderen“. Im Modell von Deardorff wird versucht, eine Zusammenführung der Theorien aus den beiden Richtungen und den unterschiedlichen Wissenszweigen vorzunehmen. In der vorliegenden Arbeit wird ein von der Verfasserin entwickeltes Puzzle-Modell der interkulturellen Kompetenz, das in den weiteren Kapiteln noch vorgestellt wird, verwendet. 


\section{Das Puzzle-Modell - ein Vorschlag für die Herausbildung der interkulturellen Kompetenz im Fremdsprachenunterricht}

In dieser Arbeit wird für die empirische Untersuchung ein Modell entwickelt, das die Kompetenz eines Menschen als eine Art Puzzle versteht, in dem unterschiedliche Kompetenzen zusammengesetzt werden können. Es werden diejenigen Kompetenzen ausgesucht, die zum Erreichen eines Ziels notwendig sind. Dabei stehen alle Kompetenzen im engen Zusammenhang zueinander. Die interkulturelle Kompetenz stellt so gesehen ein Teil des Ganzen dar, das auch aus einigen Puzzle-Stückchen zusammengesetzt wird. Dabei kann interkulturelle Kompetenz durch die anderen Kompetenzen, Fähigkeiten oder Einstellungen ergänzt werden. So zum Beispiel ergänzen die kommunikative und die Berufskompetenz die interkulturelle Kompetenz beim Gespräch zwischen zwei Geschäftsleuten, dessen Ziel der Abschluss eines Handelsvertrags ist. Oder die emotionale Intelligenz und offenes Sozialverhalten (Offenheit, Fähigkeit zur Konfliktvermeidung, Empathie ...) verhelfen einem Menschen zu einem friedlichen Zusammenleben mit Vertretern anderer kultureller Kreise. Wenn wir ein Puzzle-Stückchen aus dem gesamten Puzzle-Bild herausnehmen, wird das Bild zwar lückenhaft, aber nicht zerstört, d.h. beim Fehlen einer Teilkompetenz bedeutet es nicht, dass der Mensch überhaupt nicht kompetent ist, sondern es fehlt ihm etwas, das beim Erreichen des Ziels helfen kann.

Bei der Kommunikation, die immer Kompetenzen von zwei oder mehreren Menschen braucht, ist es wichtig, dass möglichst viele Puzzle-Stückchen (z.B. Kompetenzen und Kenntnisse) bei den Partnern übereinstimmen. Bei einer hohen Übereinstimmungsquote ist das Ausfallen einer der Kompetenzen oder das Fehlen eines gemeinsamen Wissens in einem bestimmten Bereich nicht mehr von einer alles bestimmenden Bedeutung. Bei der interkulturellen Kommunikation, wo Partner aus unterschiedlichen kulturellen Kreisen kommen, ist die Wahrscheinlichkeit einer Übereinstimmung durch fehlendes kulturelles Wissen oder unterschiedliche Einstellungen und Werte geringer, was zu einer lückenhaften oder sogar abgebrochenen Kommunikation führen kann. In solchem Fall könnte das Puzzle-Modell mit seinen interkulturellen „Teilchen“ hilfreich sein. Sie helfen durch ihre Komponente bei einer gestörten Kommunikation.

Die Puzzle-Teilchen, die unbedingt zur interkulturellen Kompetenz gehören und zu deren Verbesserung dieses Programm entwickelt wurde, sind:

- Kenntnisse

- Fähigkeiten 
- interkulturelle Sensibilität

Diese Komponenten sind anscheinend unabhängig voneinander und von anderen Elementen der menschlichen Kompetenz, aber in der Realität sieht es wie immer anders aus: Die Komponenten sind eng miteinander verbunden, sie fließen häufig ineinander über und können auch nur miteinander und durcheinander entwickelt werden. Zu diesen drei Bestandteilen, die den Kern der interkulturellen Kompetenz ausmachen, gehören aber auch periphere Komponenten wie zum Beispiel die soziale Kompetenz, emotionale Intelligenz, Berufskompetenz, kommunikative Kompetenz und einige andere. Dieses Modell bleibt ein unvollständiges, aber es kann im Fremdsprachenunterricht eingesetzt und erprobt werden. Für die vorliegende Arbeit war das ein wesentliches Ziel der Untersuchung.

Der Prozess des Fremdsprachenunterrichts stellt in erster Linie einen Lernprozess dar, woran einerseits Lehrer und andererseits Lernende teilnehmen. Damit dieser Prozess bewusst gesteuert werden kann, ist es wichtig, ein Ziel zu setzen, das immer im Auge zu behalten ist. Es gibt dem Unterricht eine notwendige Richtung und hilft dem Lehrer zu beurteilen, ob der Prozess des Lernens stattgefunden hat.

In der vorliegenden Arbeit wird die interkulturelle Kompetenz als Ziel des Fremdsprachenunterrichts in den Mittelpunkt gestellt. Sie ist nach Köck (1995) als Richtziel zu definieren, also als Ziel eines einzelnen Unterrichtsfachs. Sie kann aber auch als Leitziel betrachtet werden, als ,,allgemeines und weitgehend zeitloses Ziel der Gesellschaft“ (Mietzel 2007, S. 427), da fast jede moderne Gesellschaft von dem Problem der wachsenden Multikulturalität betroffen ist.

Es wird hier über das Lernziel gesprochen. Obwohl solche Begriffe wie Lehrziel, Lernziel, Bildungsziel oder Erziehungsziel in den unterschiedlichen wissenschaftlichen Arbeiten anzutreffen sind, hat sich der Begriff „Lernziel“ am meisten durchgesetzt. Dies ist der Individualisierung des Unterrichts zu verdanken, wobei der Unterricht schülerzentriert betrachtet wird. „Lernziele benennen das Wissen, die Fähigkeiten und Fertigkeiten, die ein Lernender am Ende einer Unterrichtseinheit beherrschen sollte“ (Mietzel 2007, S. 427).

Wie aus der Psychologie, insbesondere aus der Lernpsychologie bekannt ist, richtet sich ein Lernprozess auf drei Hauptbereiche: den kognitiven, den affektiven und die Handlungsbereich. So unterscheidet der amerikanischen Psychologe Benjamin Bloom (1972) in einem Lernprozess drei Zielbereiche:

- kognitive Lernziele, die sich auf Wissen, Wahrnehmungs-, Gedächtnis- und Denkleistungen beziehen; 
- affektive Lernziele, die sich auf Haltungen und Werte, Interessen, Einstellungen, Gefühle richten, diese sind schwierig zu beurteilen;

- psychomotorische Lernziele, sie beziehen sich auf manuelle Fähigkeiten und andere körperliche Tätigkeiten. Sie beschreiben die Koordination von Bewegungen und Bewegungskombinationen. ${ }^{13}$

Im Jahre 2001 wurde die Taxonomie der Lernziele von Bloom einer kritischen Revision unterzogen, infolgedessen noch eine neue Dimension im kognitiven Bereich hinzugefügt wurde. Es handelt sich dabei um eine Wissensdimension. Dabei wird untersucht, ob die kognitiven Prozesse tatsächlich stattgefunden haben und ob Wissen ausgebildet wurde, das später in den weiteren kognitiven Prozessen wie Erinnern, Verstehen, Anwenden, Analysieren, Bewerten und Kreieren verarbeitet werden kann. (vgl. Anderson und Krathwohl 2001). Diese dreidimensionale Aufteilung der Lernziele aus der Lernpsychologie wurde als Grundlage für das in dieser Arbeit vorgestellte Modell der interkulturellen Kompetenz benutzt. Während die interkulturelle Kompetenz als Richtziel des Fremdsprachenunterrichts angesehen wird, stellen die kognitiven, affektiven und pragmatischen Dimensionen drei Bestandteile von ihr dar.

Für das Erreichen der kognitiven Lernziele ist die erste Komponente des Modells verantwortlich: Die Aneignung des Wissens ist ein wichtiges Ziel des Lernens. Das Wissen, das beim interkulturellen Lernen erworben wird, sind Kenntnisse über kulturspezifische Erscheinungen. Aufgrund der Kenntnisse werden später die Fähigkeiten - die zweite Komponente im Modell - ausgebildet, die den Lernenden erlauben, sich adäquat in der interkulturellen Situation zurechtzufinden. Die affektiven Lernziele finden ihre Widerspiegelung in der dritten Komponente, die als Sensibilität gegenüber interkulturellen Verhältnissen bezeichnet wird.

Die Sprachkompetenz oder die kommunikative Kompetenz in einer Fremdsprache (wie sie in der Fremdsprachendidaktik genannt wird) nimmt eine besondere Stellung ein. Obwohl sie hier als vierte Komponente nicht ausgegliedert wird, ist die sprachliche Kompetenz ein unabdingbarer Teil der interkulturellen Kompetenz, allein aus dem Grund, dass es um das Erlernen einer Fremdsprache in einer Lehreinrichtung geht. Wenn wir uns ein Puzzle vorstellen, dessen Teile die oben genannten Dimensionen sind, ist es klar, dass diese Teilchen ineinander einfließen und übergehen und nicht strikt voneinander getrennt werden können. So ist die sprachliche Kompetenz Teil dieser drei Dimensionen, sie befindet sich quasi in

13 Dabei teilt er die Fähigkeiten in zwei unterschiedliche Zielgebiete, die intellektuellen Fähigkeiten gehören dann zum kognitiven Bereich und sind getrennt von den manuellen und körperlichen. 
der Mitte, bildet sozusagen den Kern des Zieles im Fremdsprachenunterricht. Wie schon im Kapitel 1.2 erwähnt wurde, wird die interkulturelle Kompetenz als Erweiterung des ,alten“ Ziels „kommunikative Kompetenz“ betrachtet.

Es wurde im vorgeschlagenen Modell bewusst auf die in vielen Theorien erwähnte und als einer der Hauptbestandteile der interkulturellen Kompetenz in der Zusammenfassung von Deardorff benannte Komponente „Haltungen und Einstellungen“ verzichtet. Hier sind zwei wichtige Gründe dafür:

- Haltungen und Einstellungen, wie sie in den unterschiedlichen Theorien präsentiert werden, sind m.E. Bestandteile der sozialen Kompetenz. Die soziale Kompetenz bildet mit den unterschiedlichen anderen Kompetenzen, darunter auch mit der interkulturellen Kompetenz, eine Art Puzzle, die als Grundlage für das menschliche Verhalten in unterschiedlichen Situationen dient. Ohne solche Fähigkeiten wie Offenheit, Flexibilität, Empathie, Ambiguitätstoleranz wäre Verhalten in der interkulturellen Überschneidungssituation schwer zu deuten, und der Prozess der Verständigung könnte erschwert werden oder gar nicht möglich sein. Aber die Wurzeln dieser Fähigkeiten liegen grundsätzlich in den sozialen Einstellungen eines Menschen zur Welt, egal ob seine Kommunikation in einem einheitlichen oder multikulturellen Milieu stattfindet. Zur Entwicklung dieser Fähigkeiten im Umgang mit interkulturellen Situationen kann die im Modell vorgesehene Sensibilisierung beitragen. Die Sensibilisierung kann aber nicht die schon präsenten Einstellungen grundsätzlich ändern.

- Die Veränderung der Einstellungen und der Haltungen ist ein schwieriger und langer Prozess, der reichlich praktische Erfahrung fordert. Das heißt, damit die Einstellungen einer Person zu bestimmten Sachverhalten verändert werden kann, muss diese Person die Situation, in unserem Fall die Situation einer interkulturellen Begegnung, selbst erleben oder mehrmals daran teilgenommen haben. Im Rahmen eines Fremdsprachenunterrichts wäre das sehr schwer, da unter diesen Bedingungen normalerweise der Kontakt nur zu den Mitstudierenden oder zum Lehrer besteht. ${ }^{14}$ Eigene Erfahrung in der Situation eines interkulturellen Kontakts, die bewusst erlebt und analysiert wurde, ist m.E. die Grundlage für die Veränderung der Einstel-

14 Wenn ich an dieser Stelle die Situation der interkulturellen Begegnung nenne, meine ich die Begegnung zwischen Vertretern unterschiedlicher Nationen, die nicht zu einem kulturellen Kreis gehören. Im Fremdsprachenunterricht in einer Hochschule in Russland können Vertreter von unterschiedlichen Nationalitäten wie Russen, Burjaten oder Jakuten teilnehmen, sie gehören aber gleichzeitig zu einer Subkultur, sie sind alle Studierende einer Hochschule, wo fast ausschließlich Russisch gesprochen wird. 
lungen. Um die Schwierigkeiten und die Besonderheiten der interkulturellen Situation wahrnehmen zu können, muss das Thema zunächst im Unterricht angesprochen und bearbeitet werden. Deshalb wurde ein dritter Teil des Kompetenzmodells - die Sensibilisierung - festgelegt. Dazu gehören insbesondere die Einstellungen und Haltungen der Lernenden.

\subsection{Kulturspezifische und kulturübergreifende Kenntnisse als kognitive Komponente der interkulturellen Kompetenz}

Das Wort „Wissen“ wird in den unterschiedlichen Wissenschaften, aber auch in der Umgangssprache verwendet. Was bedeutet eigentlich „Wissen“? Im Allgemeinen wird Wissen als die verallgemeinerte Widerspiegelung der realen Welt im menschlichen Bewusstsein verstanden, also ein subjektives Bild der objektiven Wirklichkeit.

Wissen bedeutet „die im Gedächtnis einer Person gespeicherten und reproduzierbaren Beobachtungen, Erfahrungen, Kenntnisse und Einsichten über vielfältige Wirklichkeitsbereiche, die einerseits oft die Folge unbeabsichtigter Lernprozesse sind, andererseits aber die Voraussetzungen für bewusste Lern-, Denk- und Problemlösungsprozesse, von denen aus ein Individuum die Welt interpretiert und auf sie verändernd einwirkt.“ (Schaub und Zenke 1997, S. 369).

In der Kognitionspsychologie unterscheidet man deklaratives und prozedurales Wissen. Im Unterschied zum prozeduralen Wissen, das handlungsorientiert ist und als eine Fähigkeit, etwas durchzuführen, bezeichnet wird, stellt das deklarative Wissen, das Wissen über Fakten, Wissen über etwas dar und setzt Bewusstheit des Lernenden voraus. Die Einordnung des interkulturellen Bewusstseins (oder intercultural awareness) unter dem zusammenfassenden Begriff des Wissens, wie es im Common European Framework of Reference gemacht wurde, ist deshalb durchaus akzeptabel (vgl. Trim 2006).

Was den Lernprozess anbetrifft, wird jedoch ein anderes Wort bevorzugt, und zwar „Kenntnisse“. Während „Wissen“ ein relativ abstraktes Begriff ist, sind mit dem Begriff „Kenntnissen“ die konkreten Sachverhalte gemeint. ${ }^{15}$

Kenntnisse sind „das in Lernvorgängen erworbene Wissen über Sachverhalte und soziale Zusammenhänge“ (Schaub und Zenke 1997, S. 201). In dieser Arbeit verwendet die Ver-

15 Die Worte „Kenntnisse“ und „Wissen“ haben unterschiedliche Bedeutungen in der deutschen Sprache. Es muss aber erwähnt werden, dass es sowohl in der englischen als auch in der russischen Sprache nur ein Wort für diese beiden Begriffe gibt (engl. , „knowledge“, russ. „знания“). 
fasserin den Begriff „Kenntnisse“, weil es um konkret zu benennende, im interkulturellen Lernen zu erwerbende Sachverhalte geht. Es stellt sich die Frage, welche das im Einzelnen sind.

Die interkulturelle Realität stellt eine breite Palette von Tatsachen, Fakten und Meinungen dar. Wenn die Vermittlung interkulturellen Wissens im Unterrichtsprozess stattfindet, sind Zeitrahmen und Möglichkeiten begrenzt. Stellen wir uns die Situation einer möglichen Begegnung des Lernenden mit den Vertretern einer anderen Kultur vor, also eine sogenannte interkulturelle Überschneidungsituation. Der Lernende besitzt bereits das Wissen über die Wirklichkeit seiner Kultur. Wissen über fremde Kultur hat er nur teilweise oder es fehlt überhaupt. Der erste Teil der zu vermittelnden interkulturellen Kenntnisse muss also die Realität der fremden Kultur betreffen. Das sind die sogenannten soziokulturellen Kenntnisse, welche reichlich im Fremdsprachenunterricht in Bezug auf die Kultur der erlernten Sprache präsent sind. Dieser Aspekt des Wissens wird sowohl in der Muttersprache als auch in der Fremdsprache erlernt. Obwohl die Vermittlung der soziokulturellen Kenntnisse seit einigen Jahrzehnte stattfindet, sind sich die Theoretiker der Landeskunde oder der linguistischen Landeskunde bis jetzt nicht einig, welche Realien der fremden Kultur ihren Platz in der Unterrichtsstunde finden sollen.

Die kulturelle Überschneidungssituation funktioniert nach eigenen Gesetzen und Regeln, denn das ist ein Zwischenraum, wo die Realitäten beider Gesprächspartner gleichzeitig existieren. Das ursprüngliche Wissen über die Realität der eigenen Kultur stößt auf das fremde Wissen, das oft ganz anderer Natur ist. In diesem Fall genügen die soziokulturellen Kenntnisse über die fremdkulturellen Erscheinungen einfach nicht. Hier sind Kenntnisse über die Besonderheiten der interkulturellen Überschneidungssituation erforderlich. Diese Kenntnisse haben eine rein theoretische Natur, sie helfen den Lernenden zu verstehen, welche Prozesse in ihm selbst und in seinem fremdkulturellen Partner ablaufen und wo die Gründe für mögliche Missverständnisse liegen können. Die Aneignung dieser Kenntnisse führt zu der bewussten Wahrnehmung des eigenen und des fremden Verhaltens und der eigenen und der fremden Gefühle. Auch die besonderen Umstände, unter denen eine solche Kommunikation abläuft, werden bewusst.

Bei dem Begriff „Wissen“ unterscheidet z.B. Antor (2002) zwischen dem Erwerb traditioneller landes- und kulturkundlicher Kenntnisse und der Akquisition theoretischer Kenntnisse über den Umgang mit dem Phänomen der Alterität oder Andersartigkeit.

Es wird hier zwischen zwei Arten des interkulturellen Wissens unterschieden: den kulturspezifischen und den kulturübergreifende Kenntnissen. 


\subsubsection{Die kulturspezifischen Kenntnisse}

Was hier als kulturspezifisches Wissen bezeichnet wird, ist in anderen Modellen unterschiedlich benannt. So bezeichnete Brislin, der sich hauptsächlich mit interkulturellen Trainingsmaßnahmen beschäftigte, diese Art von Wissen als „Area-Specific Knowledge“. Er erwähnt die Wichtigkeit des Erwerbs der Kenntnisse über die fremde Kultur, geht aber kaum darauf ein, welche Informationen über die Zielkultur vermittelt werden sollen. Das ist auch zu verstehen, denn Brislin spezialisiert sich in den kulturübergreifenden Trainings auf die Vorbereitung der Fachkräfte auf einen Auslandseinsatz. Seine Trainingsmaßnahmen sind nicht für ein bestimmtes Land gedacht.

"For trainees interacting with ethnic minorities and other culturally different people within the United States, understanding their history, sociology, economics, psychology, geographic location, socioeconomic status, educational information, employment information, and family relationships is essential" (Brislin und Yoshida 1994, S. 35).

Im gemeinsamen europäischen Referenzrahmen für Sprachen, der als grundlegendes Konzept für das Erlernen einer Fremdsprache und für die gemeinsame Beurteilung des Niveaus der Sprachbeherrschung in Straßburg 1996 ausgearbeitet wurde, wird diese Art von Wissen als soziokulturelles Wissen bezeichnet. Der Name selbst besagt bereits, dass es sich hier um Kenntnisse aus dem sozialen Bereich einer fremden Kultur handelt.

Allerdings ist jede menschliche Kultur so vielseitig und vielschichtig, dass es kaum möglich ist, jede Einzelheit des sozialen Lebens der Vertreter einer fremden Kultur im Unterricht zum Ausdruck zu bringen. Daher ist es wichtig, die Informationen zu vermitteln, die später von den Lernenden in der Situation des möglichen interkulturellen Kontakts benutzt werden können. Da meist vier Anwendungsbereiche der Sprache unterschieden werden, und zwar die Anwendung im privaten Bereich, im Beruf, in der Ausbildung und in der Öffentlichkeit, ist es notwendig, das kulturspezifische Wissen jedes Bereichs für den Lernenden präsent zu machen. Dabei müssen die sprachliche Ziele des Unterrichts beachtet werden, die manchmal schon einen Hinweis darauf geben, welcher Bereich im Unterricht bevorzugt werden sollte. Wenn z.B. ein Programm zur Entwicklung interkultureller Kompetenz für eine Fachhochschule gedacht ist, wo der berufliche Bereich der Sprachanwendung als Zielsetzung des Fremdsprachenunterrichts im Vordergrund steht, dann gelten für die Auswahl von Informationen zusätzliche Kriterien, die den Wert der Informationen für die Berufssphäre des Lernenden in Auge haben.

Die Annahme, dass bei der Auswahl des kulturspezifischen Wissens die berufliche Aus- 
richtung des Anwendungsbereichs der Sprache beachtet werden muss, erleichtert m.E. die Auswahlarbeit, da Berufsgespräche sowohl einen bestimmten Wortschatz erfordern als auch die große Menge kulturspezifischer Informationen auf einen bestimmten Bereich reduzieren.

Während die kommunikativen Bedürfnisse des Lernenden beim berufsspezifischen kulturellen Wissen vorhersehbar sein können, sind sie in den persönlichen und sozialen Bereichen des Sprachgebrauchs nicht so einfach zu bestimmen. ${ }^{16}$ Es ist schwer einzuschätzen, welche Informationen aus der fremden Kultur der Lernende benötigen wird, da er in unterschiedlichen Lebenssituationen des interkulturellen Kontakts ganz unterschiedliche kommunikative Aufgaben zu lösen hat. Reichen die Informationen über Tatsachen und Fakten über das fremde Land aus, so wie zum Beispiel Kenntnisse über die geografische Lage, die Größe und die Fläche des Landes, über Traditionen, Bräuche und Feste, über die Spezialitäten und klassische Literaturstücke? Die Antwort auf diese Frage hängt natürlich von der Art der Kommunikationen ab, aber auch davon, welche Ziele die Gesprächspartner verfolgen. Es ist jedoch mit Sicherheit anzunehmen, dass sie bei wiederholten Kontakten oder bei der Lösung einer gemeinsamen Aufgabe nicht ausreichen werden.

Weshalb allgemeine Informationen bei den realen interkulturellen Kontakten nicht ausreichen, liegt in der Natur der Kultur selbst. Die nationale Kultur ist viel mehr als das, was der Mensch auf den ersten Blick sieht, wenn er ein fremdes Land besucht. Kultur ist mehrschichtig, wie es im „Eisbergmodell“ zu sehen ist (siehe Anhang 1). Das Modell beruht auf der Annahme, dass eine Kultur einem Eisberg ähnlich ist: Nur ein Teil, und zwar der kleinste, ist über dem Wasser zu sehen, der größte Teil, der auch mehr Gefahren mit sich bringt, bleibt dem ersten Blick verborgen. Beim ersten Kontakt mit einer fremden Kultur, sei es beim Besuch eines anderen Landes oder beim Gespräch mit einem seiner Vertreter, bekommen die Personen nur die sichtbaren Teile zu sehen, wie zum Beispiel Essensgewohnheiten, Kleidung oder Unterhaltungskultur. Die wichtigsten Informationen bleiben verborgen, es sind aber gerade jene, die als Fundamente oder Grundlagen für das Verhalten fremdkultureller Menschen gelten. Dazu gehören z.B. Herrschaftsverhältnisse; die Aufteilung der sozialen Rollen nach Alter, Geschlecht, Beruf; Arbeitstempo und Verhältnis zur Zeit; Körpersprache und Mimik; Problemlösungsverhalten; Gefühls- und Verhaltensmuster und noch vieles andere.

16 Bewusst erwähne ich die als vierten Sprachgebrauchsbereich genannte Ausbildungssphäre nicht. Die Ausbildung oder Lehre bedeutet die Vorbereitung $\mathrm{zu}$ einem bestimmten Beruf, die Ausbildung ist grundsätzlich auf berufliche Begriffe und Inhalte gerichtet, deshalb stehen die beiden Sprachgebrauchsbereiche relativ nahe beieinander. 
Bei der Auswahl des kulturspezifischen Wissens müssen die sich unter der Oberfläche befindlichen Teile der Informationen über die fremde Kultur beachtet werden, weil sie einerseits von den Lernenden selbst schwer erkannt werden können, andererseits eine entscheidende Rolle für den Erfolg der interkulturellen Kommunikation spielen.

Die Basis des Eisberges bilden die sogenannten kulturellen Normen. Diese Normen sind das Ergebnis der Erziehung in einer bestimmten Kultur, sie sind beim ersten Treffen mit den Vertretern dieser Kultur kaum erkennbar. Diese Normen stellen bestimmte Regelungen für das Verhalten der Menschen dar.

Normen können mit einer Karte oder einem Schema des kulturellen Lebens verglichen werden. Normen liefern Verhaltensmuster, die sich die Mitglieder einer Kultur im Prozess der Inkulturation lernen und die zum Bestandteil ihrer Persönlichkeit werden.

Kulturelle Normen sind die gesellschaftlich anerkannten Vorschriften, Forderungen, Wünsche und Erwartungen an ein Individuum, was sein Verhalten betrifft. Die Inhalte der Normen bilden gewisse idealisierte Vorschriften, wie die Menschen in bestimmten Situationen sprechen, denken, fühlen und einhalten sollen (Kravtschenko 2000, S. 74).

Es gibt kulturelle Normen, die für die meisten Menschen grundsätzlich ähnlich oder gleich sind. So werden zum Beispiel Totschlag oder Diebstahl in modernen Gesellschaften als zu bestrafendes Verhalten bewertet. Am meisten sind die Normen durch die Erziehungskultur geprägt. Für Menschen aus anderen Kulturen sind sie meist unverständlich. Es fällt dem Fremden oft nicht auf, dass er die Erscheinungen einer anderen Kultur mit den eigenen Normen interpretiert und bewertet. Lächeln bedeutet z.B. für einen Europäer in der eigenkulturellen Situation eine Zustimmung, genauso wird das Lächeln eines japanischen Gesprächspartners dann von diesem Europäer interpretiert, was zu einem Missverständnis bei Geschäftsgesprächen führen kann. Denn das Lächeln ist in der japanischen Kultur ist Lächeln ein Zeichen der Verlegenheit.

Den Verstoß gegen eine Norm belegt die Gesellschaft mit einer Strafe. Wie schwer diese Strafe sein wird, hängt von der Norm ab. So gibt es kulturelle Normen, deren Verstoß Missbilligung, Grinsen, bösen Blick, Missverständnis, fehlgeschlagene Kommunikation hervorruft. Ein Ausländer, der in einer Gastfamilie im Ausland lebt und sich gegen die Regeln dieser Familie benimmt (meist unbewusst, weil er sich so verhält, wie er es aus der eigenen Familie kennt), muss mit der Missbilligung des Gastgebers rechnen. Beim Verstoß gegen andere Normen sind schlimmere Folgen zu erwarten, wie Festnahme oder sogar Todesstrafe. Ein Beispiel hierzu ist der Fall von Marco W. ${ }^{17}$, der durch sein Benehmen angeb-

17 Marco Weiss ist ein deutscher Staatsbürger, dem im Sommer 2007 vorgeworfen wurde, während eines 
lich gegen jegliche Regeln und Gesetze der türkischen Kultur verstoßen hat und dadurch eine Strafe erlitt, die ihm und seinen Angehörigen als ungerecht schwer erscheinen musste, da sie mit den Normen ihrer heimischen deutschen Kultur denken und urteilen.

In der Abbildung 5 sind Normen in Abhängigkeit von dem Härtegrad der vorgesehenen Strafe, die ein Verstoß nach sich zieht, dargestellt. Oben sind die Normen, die eine härtere, unten die Normen, die eine mildere Strafe bzw. Toleranz gegenüber Abweichungen nach sich ziehen. Die Achse X zeigt den Verbreitungsgrad dieser Normen. Je milder die Strafe für den Verstoß gegen eine kulturelle Norm ist, desto verbreiteter ist diese Norm in der Gesellschaft.

Abbildung 5: Gesellschaftliche Normen

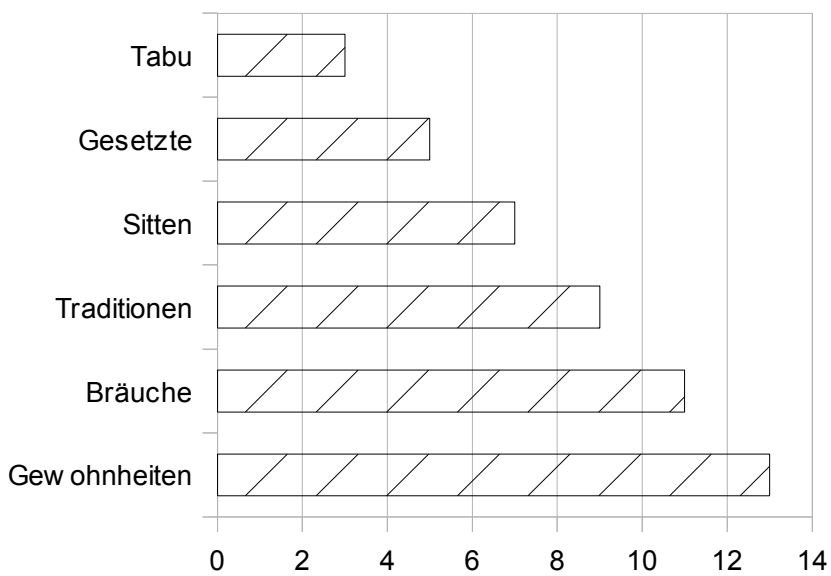

Ganz oben in dieser Hierarchie befinden sich die Tabus, d.h. es handelt sich um ein absolutes Verbot, was eine Handlung, ein Wort oder eine Sache angeht. In den modernen europäischen Gesellschaften sind solche Erscheinungen wie Kannibalismus, Inzest oder Gräberentweihung ein Tabu. Der Verstoß gegen diese Normen hat meistens schlimme Folgen.

Es gibt aber auch tabuisierte Gesprächsthemen oder Redewendungen, deren Verwendung manchmal zum Abbruch oder zur Gefährdung des Gesprächs führen kann. In der amerikanischen Kultur z.B. sind bestimmte Themen, die den konfliktlosen Gesprächsablauf schädigen können, tabuisiert; deshalb werden solche Themen wie Politik oder Religion bei privaten Gesprächen nicht gern gesehen, da sie der Gefahr von Meinungsunterschieden ausgesetzt sind, die leicht zu Streitigkeiten führen können.

Urlaubes in der Türkei ein Kind (ein 13-jähriges Mädchen aus England) missbraucht zu haben. Dem Vorwurf folgte eine achtmonatige Untersuchungshaft und anschließend die Verurteilung. 
Die Normen und Verhaltensregeln, die in einem parlamentarischen Dokument verfestigt sind, werden Gesetze genannt, sie sind streng zu erfüllen und ziehen bei Nichtbeachtung eine gesetzlich anerkannte Strafe nach sich. Sie stehen auch ganz oben in der angeführten Normenhierarchie.

Die nächste Stufe nehmen „Sitten und Gebräuche“ ein. Sie stellen die üblichen Verhaltensmuster dar, die besonders streng von der Gesellschaft aufbewahrt werden. Die Bedeutung der Sitten ist mit den moralischen Prinzipien und den ethischen Normen verbunden.

Aus allen kulturellen Normen sind die Sitten besonders flexibel, da sie die laufenden Ereignisse und Taten regulieren sollen. „Sitten sind die moralischen Bewertungen, die bestimmte Formen des eigenen Verhaltens und des Verhaltens der anderen zulassen. Unter die Wirkung dieser kulturellen Normen fallen diejenigen Verhaltensformen, die für eine bestimmte kulturelle Einheit üblich sind, die Zugehörigkeit stiften bzw. erkennbar machen“ (Gruschevskaja, Popkov und Sadochin 2002, S. 47).

Wie aus dem oben angeführten Zitat zu ersehen, sind gerade die Sitten kulturell geprägt, sie sollten daher bei der Auswahl des soziokulturellen Wissens berücksichtigt werden. Nehmen wir als Beispiel die Situation und die gesellschaftliche Meinung über Altenwohnheime in Deutschland und Russland. Wenn alte Menschen in Deutschland nicht mit den Kindern, sondern in einem Altersheim leben, akzeptiert die Öffentlichkeit die Situation als völlig normal. Die gleiche Situation wird in der russischen Kultur als Abweichung wahrgenommen, weil in Russland die Familie für die alten Menschen sorgen muss ${ }^{18}$. Den Kindern würde in dem Falle seitens der Öffentlichkeit das Gefühl vermittelt, sich nicht anständig, weil normabweichend, verhalten zu haben.

Als weitere Elemente der kulturellen Normen sind Traditionen und Bräuche zu nennen. Gerade diese Verhaltensweisen scheinen in den Lehrbüchern am meisten präsent zu sein, der Umfang dieser Information ist wesentlich breiter als der anderer Verhaltenstypen. In den meisten Lehrbüchern sind z.B. die Traditionen einer Weihnacht- oder einer Osterfeier, das traditionelle Essen, Trinken oder Freizeitaktivitäten beschrieben. Die soziale Strafe für Abweichung von diesen Normen ist nicht so gravierend streng wie bei Tabus oder Sitten, kann aber zu Missverständnissen und dadurch zu negativen Emotionen führen. In den meisten europäischen Kulturen sind Blumen bei Festlichkeiten, bei Geburtstagen oder Hochzeiten willkommen. Wenn aber ein Gast in Russland eine ungerade Zahl von Blumen

18 Es wird jetzt absichtlich auf die unterschiedlichen Bedingungen in den Wohnheimen beider Länder nicht eingegangen. Die Autorin schätzt die Situation jedoch folgendermaßen ein: Das System der sozialen Vorsorge ist in Deutschland deshalb besser aufgebaut, weil es sich den Einstellungen und Bedürfnissen der Gesellschaft anpasst. In Russland dagegen sind die schlechten Bedingungen nicht nur die Folge der sozialen Schwäche, sondern auch Folge der oben genannten Einstellungen. 
schenkt, wird es als Beleidigung wahrgenommen, da eine ungerade Blumenzahl mit der Trauerfeier verbunden ist. Die allgemeine Tradition „Blumen schenken“ korreliert hier mit dem russischen Brauch, der die Zahl oder die Art der Blumen betrifft. In Deutschland dagegen gilt eine gerade Zahl als langweilig, wenig geschmackvoll.

Gewohnheiten sind Verhaltensstereotype, die infolge einer mehrfachen Wiederholung ausgebildet wurden. Von sich aus ist eine Gewohnheit seitens der Gesellschaft nicht strafbar. Es gibt aber Gewohnheiten, die gegen Manieren verstoßen, was eine negative Reaktion der kulturellen Umgebung nach sich zieht. Der Begriff ,gute Manieren“ ist stark kulturell geprägt: das, was in einer Kultur für „gut“ gehalten wird, wird in einer anderen Kultur als Verletzung von Normen interpretiert. Das Aufstoßen nach dem Essen ist, wie alle Arten von Essgeräuschen, in den meisten modernen europäischen Kulturen „,nicht schön, ungehörig bis unanständig“. Diese Einschätzung gilt nicht für mongolische oder burjatische Kulturen. Das Aufstoßen würde dort als ein Zeichen interpretiert, dass der Mensch satt ist und dass das Essen ihm geschmeckt hat.

Dabei muss aber erwähnt werden, dass Abweichungen zwischen Normen zweier unterschiedlicher Kulturen im unteren Bereich am stärksten sind. Verwandte Kulturen, die entweder geografisch nicht weit voneinander entfernt liegen oder eine gemeinsame Geschichte haben, werden höchstwahrscheinlich gleiche oder ähnliche Tabus, Gesetze und Gebräuche haben.

Alle Normen existieren nicht getrennt voneinander, sondern sind in ein Wertesystem eingebunden. Dieses Wertesystem ist für die Augen eines Fremden nicht unmittelbar erkennbar, aber es dient als Grundlage für die Vermittlung der sichtbaren, erlernbaren Komponenten einer fremden Kultur. Der Wert stellt eine Einheit dar, die sich schwer mit Worten fassen lässt. Die Rahmenbedingungen von Unterricht, die künstlich geschaffene Atmosphäre der Fremdsprachenstunde lassen weder genügend Zeit noch ausreichend Möglichkeiten, Werte einer fremden Kultur zu erlernen. Der Ausweg für die Fremdsprachendidaktiker ist, die sich im oberen Teil des Eisbergmodells befindenden Komponenten einer Kultur, deren Sprache erlernt wird, zu vermitteln. Die Ausarbeitung eines gemeinsamen europäischen Referenzrahmens für Sprachen haben diese Komponenten unter dem Titel „Soziokulturelles Wissen" vereint und in sieben Unterkategorien aufgeteilt. ${ }^{19}$ Die Merkmale, die für eine

19 Es ist dabei zu beachten, dass die oben genannten Rahmen für den Bereich „europäische Sprachen“ gelten. Sie können im Prinzip auf andere Sprachen wie z.B. asiatische übertragen werden. Diese Rahmen sollten aber dann m. E. mit Vorsicht behandelt werden. Bei der Beschreibung des ,soziokulturellen Wissens" wurden wahrscheinlich unterschiedliche Kulturtheorien bearbeitet. Obwohl sich diese Theorien an allen Kulturen orientieren, sind sie meist ,westlich“ geprägt, allein aus dem Grund, dass ihre Urheber aus den westlichen Kulturen stammten. Es kann zu folgendem Nachteil führen: Die Merkmale, die aus 
bestimmte europäische Gesellschaft und ihre Kultur charakteristisch sind, können sich beziehen auf:

1. das tägliche Leben. Dazu gehört alles, wodurch der Alltag in einer Kultur geprägt ist und was als Erstes ins Blickfeld des Vertreters einer anderen Kultur gerät, z.B. die Feiertage, das unbekannte Essen und Trinken, die von den Angewohnheiten der Mutterkultur abweichenden Arbeits- und Esszeiten, die Bedeutung der Medien und der durch sie verbreiteten Informationen. In diesem Bereich gibt es reichlich Gelegenheit, ins Fettnäpfchen zu treten. Auf das unbekannte Essen kann man neugierig und gespannt sein, aber wie es ,gegessen wird“, stellt häufig ein Problem dar.

2. Lebensbedingungen. Es geht hier nicht nur um Wohnverhältnisse, sondern auch um Wohnstandards, die für die unterschiedlichen Subkulturen charakteristisch sind. Der Lernende muss erkennen, dass die fremde Kultur nicht homogen ist und dass die Werte und Einstellungen abhängig von den regionalen, ethnischen oder schichtspezifischen Gruppen variieren. So fehlt z.B. der durch Stereotype geprägten Meinung, dass alle Deutschen Lederhosen tragen und Weißwurst essen, Wissen über die Unterschiede in der Kleidung sowie über Essgewohnheiten der Bewohner der unterschiedlichen Bundesländer. Soziale Abweichungen und die damit verbundenen unterschiedlichen Möglichkeiten müssen auch in Betracht gezogen werden.

3. Interpersonale Beziehungen. Diese Merkmale beziehen sich auf die Struktur und den Aufbau der Beziehungen zwischen den Menschen. In jeder Gesellschaftsform wirken solche Merkmale wie Geschlecht, Gruppenzugehörigkeit, Alter, sozialer Status der Sprechenden auf den Ablauf der Kommunikation. Welchen Einfluss sie in der Zielkultur haben und welches Gewicht jede einzelne ihrer Komponenten in der kommunikativen Situation hat, sind die Fragen, die bei der Vermittlung dieser Kenntnisse im Vordergrund stehen sollen. Dabei kommen noch die Umstände ins Spiel, unter denen die Kommunikation abläuft, sei es die familiäre Umgebung, wo der Grad der Vertrautheit und der Informalität wahrscheinlich relativ hoch liegt, oder die öffentlichen bzw. Arbeitsbedingungen, die durch bestimmte Machtverhältnisse geprägt sind. Sie haben auch einen großen Einfluss bei der Positionierung der Gesprächspartner.

4. Werte, Überzeugungen und Einstellungen. Bei dieser Merkmalsgruppe wurde versucht, das breite Spektrum der „unsichtbaren“ Kulturkomponenten auf die wich-

der europäischen Sicht als ,wichtig“ erscheinen, könnten z.B. in den asiatischen oder afrikanischen Kulturen nicht wichtig sein oder sogar überhaupt kein Äquivalent finden. 
tigsten Faktoren zu reduzieren. $\mathrm{Zu}$ den bedeutsamen Themenbereichen gehören:

- die soziale Schicht;

- Berufsgruppen (Akademiker, Management, Beamte, gelernte und ungelernte Arbeiter);

- Vermögen (verdient und ererbt);

- regionale Kulturen;

- Sicherheit;

- Institutionen;

- Tradition und sozialer Wandel;

- Geschichte, insbesondere herausragende historische Persönlichkeiten und Ereignisse;

- Minderheiten (ethnische, religiöse);

- nationale Identität;

• andere Länder, Staaten, Völker;

- Politik;

- Kunst (Musik, bildende Künste, Literatur, Drama, populäre Musik und Lieder);

- Religion;

- Humor.

(Quelle: Gemeinsamer europäischer Rahmen für Fremdsprachen (vgl. Trim 2006)

5. Körpersprache. Mimik und Körpersprache stellen wichtige Bestandteile einer Kommunikation dar, die relativ lange Zeit von den Fremdsprachendidaktiker unterschätzt wurden. Nicht zufällig wurden die Körperbewegungen „Sprache“ genannt, da sie eine informationsübertragende Funktion haben. Wir können manchmal Information vermitteln, ohne den Mund aufzumachen, z.B. mit einem Kopfnicken für die Bejahung oder mit einem Kopfschütteln für die Verneinung. Das Körpersprachesystem ist aber genauso kulturspezifisch wie die Sprache, was manchen Leuten, die relativ wenig interkulturelle Kontakte erlebt haben, kaum bewusst ist. So z. B. die oben erwähnten Kopfbewegungen in Bulgarien oder Indien eine gegensätzliche Bedeutung haben: ein Kopfschütteln bedeutet „Ja“ und ein Kopfnicken „Nein“.

6. Soziale Konversationen. Dabei werden Informationen vermittelt, die bei der Herstellung und Aufrechterhaltung der sozialen Kontakte notwendig sind. Bei einer einmaligen interkulturellen Konversation, besonders aber bei einem lange beste- 
henden interkulturellen Kontakt (eine Freundschaft oder ein Arbeitsverhältnis) spielt jede „Kleinigkeit“ eine besondere Rolle: wie pünktlich soll die Person sein, was muss bei einem bestimmten Anlass angezogen werden, was ist bei einem Besuch mitzubringen, wann muss der Besuch beendet werden und vieles mehr. Bei einigen Kulturen gibt es auch die Tradition des Small Talk, wie und in welchen Situationen diese Art der Konversation ins Spiel kommt, ist auch wichtiger Bestandteil des Wissens. Eine besondere Gruppe dabei bilden tabuisierte Themen, Gesten und tabuisiertes Benehmen, die zum Abbruch der Konversation führen können.

7. Rituelles Verhalten. Bei dieser Merkmalsgruppe wird Wissen über Verhaltensrituale bei besonderen Ereignissen wie z.B. bei der Geburt, der Heirat, dem Tod oder bei religiösen Bräuchen vermittelt. Diese Anlässe sind m. E. besonders stereotypisiert, d.h. sie verlaufen nach einem bestimmten Schema, dessen Regeln festgelegt sind. Diese Anlässe stellen für die Einheimischen besondere Situationen dar, wobei auf die Einhaltung der Regeln besonderer Wert gelegt wird. Gerade deshalb sollen das Verhalten und die Worte eines Fremden besonders sensibel und vorsichtig sein. Durch die Rituale sind aber auch solche Feierlichkeiten wie Festivals, öffentliche Veranstaltungen, Volksfeste oder Diskotheken gekennzeichnet.

Das soziokulturelle Wissen im gemeinsamen europäischen Referenzrahmen, das mehr oder weniger aus den Cultural Studies entnommenen wurde, stimmt zum Teil mit den im Rahmen der linguistischen Landeskunde für Russisch als Fremdsprache ausgearbeiteten Informationen überein. Während die europäische Fremdsprachendidaktik landeskundliche Kenntnisse als einen expliziten Teil der in der Unterrichtseinheit mitgeteilten Informationen betrachtet, vertritt die russische Didaktik die Ansicht, dass die landeskundlichen Erscheinungen schon in der Sprache liegen und deshalb implizit beim Erlernen der Sprache angeeignet werden können.

Deshalb wird hier im Folgenden weiter über kulturspezifisches Wissen in der Sprache gesprochen.

Kulturspezifisches Wissen in der Sprache schließt folgende Elemente ein:

- verbale Komponente des Grundwissens

- verbale Etikette

- rituelle Etikette

- lexikalische Lakunen ${ }^{20}$

20 Als Lakune wird in der Philologie eine Verstehenslücke bezeichnet, die bei der Kommunikation zwischen den Vertretern verschiedener Kulturen und Sprachen entsteht. Lakunen liegen Unterschiede sowohl in den 
- Phraseologismen

Im Fremdsprachenunterricht herrscht die Atmosphäre der fremden Kultur, wo die oben genannten Merkmale, aber auch teilweise Werte und Einstellungen in den begleitenden Bedingungen wie Anschaulichkeit oder Gespräche mit authentischen Muttersprachlern zu erkennen sind. Die zu erlernende Sprache selbst bietet reichlich Informationen, sie selbst ist die Hauptquelle des kulturspezifischen Wissens. Die kulturellen Normen, die im Kontext einer Sprachgemeinschaft eingeflochten sind, werden von den russischen Linguisten und Fremdsprachendidaktikern (Werestschagin und Kostomarov 1983, Tomachin 1988, Sorokin 1982) als Grundwissen bezeichnet. Dieses Grundwissen schließt sowohl einen sprachlichen als auch einen normativen Aspekt ein. Es wird im Prinzip als sprachlicher Ausdruck der im Eisbergmodell unter der Oberfläche befindlichen Informationen über eine Kultur interpretiert. Das sprachliche Grundwissen wird selten oder wohl kaum zum Thema des Gesprächs, dient gleichzeitig aber als Grundlage eines Gesprächs, dessen Beherrschung von allen Gesprächspartnern für eine erfolgreiche Kommunikation erforderlich ist.

Furmanova (1993, S. 60) unterscheidet drei Komponenten dieses Wissens: die verbale Komponente, die verbale Etikette und die rituelle Etikette. Bei der verbalen Komponente geht es um die sprachlichen Einheiten, die gleichzeitig einen kulturellen Wert tragen. Zum Beispiel beinhalten Worte, die verschiedene in Deutschland gefeierte Feste bezeichnen (Weihnachten, Ostern, Muttertag), auch einen Komplex von Bedeutungen, Verhaltensmustern und Werten. Diese Einheiten, also quasi die Einzelteile des Grundwissens, werden hier Sprachrealien genannt.

Die verbale Komponente schließt solche Realien ein, welche die nur für diese sprachliche Gemeinschaft charakteristischen kulturellen und gesellschaftlichen Erscheinungen benennen. Die Einmaligkeit dieser Realien ist so eindeutig, dass sie die Zuschreibung dieser Erscheinungen zum kulturellen Erbe anderer Völker nicht zulassen. Dazu gehören nicht nur Realien aus dem historischen oder gesellschaftlich-politischen Bereich, sondern auch aus der Ethnographie (Wohnung, Kleidung, Feste, Tänze, Volkslieder).

In die zweite Komponente „,verbale Etikette“ werden solche verbalen Normen eingeschlossen, die sprachliche Verhaltensmuster darstellen und den Sprechenden erlauben, sich ,richtig“ in der Situation eines interkulturellen Kontakts zu benehmen. Ein ,richtiges“ Sprachverhalten stellt dabei ein „Mikrosystem der nationalspezifischen verbalen Einheiten“ dar, die in der jeweiligen Gesellschaft angenommen und den Gesprächsteilnehmern von dieser Gesellschaft vorgeschrieben sind, damit die Konversation aufrechterhalten wird und das

kulturspezischen Ansichten, als auch in den Sprachsystemen zugrunde. 
Gespräch nach den gültigen Regeln im richtigen Ton abläuft“ ( Furmanowskaja und Akischina 1982, S. 12). Die nonverbalen Kommunikationsmittel wie Distanz, Gesten, Mimik, Intonation, Sprechtempo fallen auch in den Bereich der verbalen Etikette.

Die dritte Komponente, rituelle Etikette, ist an Verhaltensmustern orientiert, die in der Gesprächsetikette verankert sind. Die Kenntnis dieser Muster und die Beherrschung der Kommunikationsstrategien erlauben den Sprechenden, eine bestimmte kommunikative Strategie zu entwickeln, die das Gespräch in die für den fremdsprachigen Kommunikanten notwendige Richtung steuert. Die Effizienz des kommunikativen Prozesses hängt unter anderem auch von solchen Faktoren wie dem psychischen Zustand der Kommunikationspartner, ihren Charakteren, ihren Einstellungen ab. Unter der Beherrschung der kommunikativen Strategien versteht Furmanova die Fähigkeit, alle diese „Kleinigkeiten“ nicht nur ,im Auge zu behalten“, sondern auch für die Realisierung seines Gesprächszieles zu nutzen. Durch die Nutzung der Etikette, die dabei als Ausdruck allgemein geltender Ideen und Werte einer anderen Kultur betrachtet wird, wird versucht, einen optimalen Einfluss auf den Partner zu gewinnen.

Die Gliederung des soziokulturellen Wissens, die von Furmanova vorgeschlagen wurde, enthält m.E. einige Kritikpunkte, zum Beispiel:

- Die verbale und die rituelle Etikette sind nicht strikt voneinander getrennt. In beiden Komponenten spricht die Didaktikerin über die Verhaltensmuster, die erlernt werden sollen.

- In der letzten Komponente spricht sie quasi über die Machtausübung. Diese Idee ist auch in den interkulturellen Trainings zu finden, die dazu dienen, Geschäftsleute auf den Auslandsaufenthalt vorzubereiten. Im Fremdsprachenunterricht jedoch kann Machtausübung als Aufgabe nach Meinung der Verfasserin ausgeschlossen werden.

Trotz dieser Kritikpunkte ist der Gedanken entscheidend, dass im Fremdsprachenunterricht das kulturspezifische Wissen von der Sprache nicht getrennt werden kann. Die Werte und Einstellungen, deren Vermittlung angestrebt wird, sind nicht nur in den Informationen aus Texten oder aus Veranschaulichungen enthalten. Sie sind auch in den sprachlichen Einheiten versteckt, sollen deshalb durch die Sprache und in der Sprache erlernt werden. Nach Werestschagin und Kortomarov besitzen die Sprache und deren Einheiten eine kumulative Funktion (vgl. S. 94), d.h. in den Spracheinheiten ist die Erfahrung mehrerer Generationen einer nationalen Kultur gespeichert. In den Wörtern selbst und besonders in den festen Redewendungen sind die Grundwerte gesammelt, die „,mit der Lebensweise und den Gedan- 
ken der Mehrheit der Menschen übereinstimmen“ (Anikin 1966, S. 3).

Jedes Wort hat nicht nur eine konkrete Bedeutung. Es ist auch im Bewusstsein der meisten Vertreter einer Kultur mit einem Kreis von Begriffen und weiteren lexikalischen Einheiten verbunden. Die Gesamtheit aller anderen Einheiten, die außerhalb der konkreten Bedeutung zu einem Wort gehören, wurde „lexikalisches Grundwissen“ genannt. „Die Universität“ und „университет“ bedeuten zwar beide eine Lehreinrichtung, wo Erwachsene eine höhere Ausbildung bekommen, haben aber relativ unterschiedliches lexikalisches Grundwissen. Im Russischen gehören folgende Merkmale dazu: fest geformte Gruppen, die hauptsächlich fünf Jahre am Unterricht nach einem für alle vorgegebenen Plan teilnehmen. Das deutsche Grundwissen zum Wort „Universität“ beinhaltet ein (für einen Russen) schwieriges System der Ausbildung, das auf der freien Wahl der Unterrichtsfächer und -zeiten gegründet ist, mit einem anderen System von Scheinen und Abschlüssen. Dieses Beispiel zeigt, dass die lexikalischen Hintergründe der auf den ersten Blick gleichen Worte nicht übereinstimmen. Wenn zu dem lexikalischen Hintergrund des deutschen Wortes folgende Worte wie Prüfungsamt, Studienberatung, Schein, Credit Points, Anmeldung zur Prüfung, Studienzentrale gehören, sind diese Worte in dem Grundwissen zu dem russischen Wort nicht präsent. Obwohl der Begriff „lexikalisches Wissen“ schon in seinem Namen auf die lexikalische Seite der Sprache verweist, enthält das Grundwissen eines Wortes viel mehr als nur eine Reihe von passenden Lexemen. Wenn bei der Semantisierung eines Wortes auch das Grundwissen zu diesem Wort erlernt wird, bekommt der Lernende nicht nur eine Menge systematisiertes kulturspezifisches Wissen, sondern auch eine Vorstellung über die fremden Strukturen, Verhaltensregeln und Normen. Wenn der russische Muttersprachler bei der Semantisierung des Wortes „Universität“ im Deutschunterricht das Grundwissen dieses Wortes nicht außer Acht lässt, bekommt er eine einheitliche Vorstellung über das deutsche Hochschulsystem, über Verhaltensregeln und Anforderungen in den deutschen Universitäten. Der Vergleich beider Universitätssysteme ermöglicht auch das Verstehen spezifischer Normen. Während z.B. das russische Hochschulsystem die Befolgung von Regeln fordert und kontrolliert, ist das deutsche System mehr auf die Selbstständigkeit, Selbstverantwortung und Aktivität der Studierenden ausgerichtet.

Lexikalisches Grundwissen ist keine rein individuelle Sache, sondern „eine Erscheinung des gesellschaftlichen sprachlichen Bewusstseins“ (Werestschagin und Kostomarov 1983, S. 58). Wie der Sprechende einen Satz aufbaut, hängt von ihm selbst ab. Seine Möglichkeiten, die einzelnen Wörter miteinander zu verknüpfen, sind aber durch Regeln eines Sprachsystems begrenzt. Hier ist ein Beispiel (vgl. Werestschagin und Kostomarov 1983, S. 77): 
Die Worte „der Junge, kommen, das Haus“ können unterschiedlich verbunden werden, „Der Junge kommt aus dem Haus “, „Der Junge kommt zum Haus“. Es kann aber kaum die folgende Verbindung geben: „Der Junge kommt unter dem Haus hervor“, weil das außerhalb der Grenzen der vernünftigen Kombinierbarkeit der Worte liegt. ${ }^{21}$

Lexikalisches Grundwissen ist keine konstante Erscheinung, es entwickelt sich genauso, wie sich die Kultur der jeweiligen sprachlichen Einheit entwickelt. Die führende Rolle wird dabei der Kultur zugewiesen, sie bestimmt das Tempo und die Art der Veränderungen, die im Grundwissen eines Wortes ablaufen. Die Dynamik der Veränderungen ist relativ hoch; die lexikalischen Hintergründe der meisten Schlüsselwörter scheinen stark verändert zu sein (vgl. Werestschagin und Kostomarov 1983, S. 79).

Wenn lexikalisches Grundwissen mit den Worten in der Muttersprache und in der erlernten Sprache nicht übereinstimmt, entstehen lexikalische Lakunen. (vgl. Sorokin 1982). Das Wort „Lakune“ wurde der medizinischen Fachsprache entnommen, wo es der hohle Zwischenraum, die Spalte zwischen den menschlichen Organen bedeutet. Genauso entstehen bei dem Erlernen einer Fremdsprache Lücken, die mit den Worten aus der Muttersprache nicht übersetzt werden können. Das sind quasi für den Lernenden „,weiße Flecken auf der Landkarte der Sprache“ (Stepanow 1985, S. 160).

„Die Lakunen sind Zeichen dafür, dass im sprachlichen und außersprachlichen Verhalten und sogar im sprachlichen Bewusstsein der Vertreter der jeweiligen Kultur national-spezifische Elemente existieren“ (Narognaja o.J.).

So ist z.B. eine „Schultüte“, die jedes deutsche Kind zu seinem ersten Schultag bekommt, eine Lakune für jeden Russen, da es weder diese Tradition noch dieses Wort im Russischen gibt.

Lakunen können absolut und verhältnismäßig sein. Bei den absoluten Lakunen sprechen wir über Erscheinungen und Worte einer fremden Kultur, die überhaupt keine Gegenbegriffe in der Muttersprache haben, ihre Bedeutung kann nicht verstanden werden. Dazu gehört auch der Wortschatz, der keine Übereinstimmung in der Fremdsprache hat (vgl. Werestschagin und Kostomarov 1983, S. 60), das sind die Begriffe aus der Geschichte, Volkskunde oder Folklore benennt. Lakunen können aber auch verhältnismäßig sein. Hier sind zwei Beispiele aus der Gestensprache. Obwohl Gesten nicht direkt zur Sprache gehören, sind sie ein wichtiges Element jeder Kommunikation und werden als nonverbale Elemente bezeich-

21 Das Beispiel gilt aber nur für die Kulturen, wo die Häuser fest auf dem Boden stehen. Was die Autoren des Beispiels nicht überlegt haben, ist die Tatsache, dass es Kulturen gibt, wo sich die Häuser über den Boden befinden, wie z.B. die maldivische Kultur. In diesen Sprachen wird sicherlich die oben genannte Kombination der Worte erlaubt sein. 
net. Sowohl in der russischen als auch in der deutschen Sprache kann man mit den Fingern zählen, aber wie es praktiziert wird, ist äußerst unterschiedlich. Während man in der russischen Kultur die gespreizten Finger einer nach dem anderen zur Mitte der Handfläche biegt (man beginnt dabei mit dem kleinen Finger), streckt man die Finger in der deutschen Kultur aus dem geballten Faust nach außen (man beginnt dabei mit dem Daumen). ${ }^{22}$ Einerseits existiert die Tradition, mit den Fingern zu zählen, in beiden Sprachen, andererseits wird dies unterschiedlich realisiert. Genauso wie bei dem Ausdruck „die Hände über dem Kopf zusammenschlagen“, was eine Geste der Bewunderung oder der Angst bedeutet. Eine ähnliche Geste gibt es dafür in der russischen Kultur, die Hände werden aber nicht über dem Kopf, sondern vor der Brust zusammengeschlagen.

Eine besondere Rolle bei der Vermittlung des kulturspezifischen Wissens durch die Sprache spielen phraseologische Redewendungen und Aphorismen.

„Phraseologismus, Redensart, Redewendung - feste sprachliche Wendungen, deren Gesamtbedeutung sich nicht mehr aus den Bedeutungen der einzelnen Komponenten ergibt, sondern die neue Umdeutung (Übertragung) erfahren hat. Die ursprünglich vorhandene Motivation geht verloren. Die Glieder eines Phraseologismus sind nicht oder nur begrenzt austauschbar“" (Brockhaus 1996, Band 17, S. 135).

Gerade die Redewendungen erfüllen infolge ihrer besonderen Struktur und der in ihre Bedeutung eingeflochtenen Geschichte besonders gut die schon oben erwähnte kumulative Funktion der Sprache. Die Gegebenheiten aus dem materiellen, sozialen oder geistigen Leben einer jeweiligen Sprachgemeinschaft kommen in den Redewendungen zum Ausdruck, die später als einheitliche Idiome in dem Bewusstsein ihrer Mitglieder verfestigt werden. So stellen die Phraseologismen eine Art Spiegel der gemeinsamen nationalkulturellen Erfahrung und Traditionen dar.

Phraseologismen sind also als prototypischer Hort des „kulturellen Gedächtnisses“ einer Diskursgemeinschaft zu betrachten; in ihnen manifestiert sich das versprachlichte kollektive Wissen und damit das „sprachliche Weltbild“ in anschaulicher und aufschlussreicher Weise. Dementsprechend greifen viele Phraseologismen dezidiert kulturspezifische Begebenheiten auf. Da die ,Bildspender' in der Regel den direkten sozialen Praxis-, Wahrnehmungs- und Erfahrungsbereichen der jeweiligen Diskursgemeinschaft entstammen, wird für die (metaphorische) Phraseologiebildung spezielles, kulturell geprägtes Wissen akti-

22 Bei der Beschreibung dieser Geste wurde die Autorin mit einer weiteren Schwierigkeit der lexikalischen Lakune konfrontiert: Was in der russischen Sprache mit einer Wortverbindung (загибать/разгибать пальцы) benannt werden konnte, musste in der deutschen Sprache mit einem ganzen Satz geschildert werden. 
viert (vgl. Földes 2005, S. 324).

So bedeutet z.B. die Redewendung ,ausgehen wie das Hornberger Schießen“, dass die Sache erfolglos, ohne klares Ergebnis ausgeht. Die Etymologie der Redewendung führt uns ins Jahr 1519 zur Belagerung der Stadt Hornberg im Schwarzwald zurück. Die Bedeutung der Redewendung wird wie folgt begründet: die Bewohner hatten die Stadt verlassen, um dem Gegner eine Schlacht unter der Stadtmauer zu liefern. Als sie eine Staubwolke sahen, wurde ein chaotisches Feuer eröffnet. Wie sich später herausstellte, war aber kein Gegner da, der Grund für diese Staubwolke war eine Schafherde. Ein anderes Beispiel mag der Name „Buxtehude“ sein; der Name dieser kleinen niedersächsischen Stadt ist ein Bestandteil von mehreren festen Redewendungen wie „ab nach Buxtehude“, „,aus Buxtehude kommen“, „nach Buxtehude fahren“, wobei dieser Name als Bezeichnung für Provinz, Pampa, eine Stelle, die sehr weit von den zentralen Regionen entfernt ist, steht (vgl. Stepanowa 1979, Malzewa 1991).

Die Bedeutungen dieser Redewendungen sind nur mit Hilfe von Kenntnissen aus der deutschen Geschichte und der Geografie zu verstehen, d.h. dass diese Redewendungen nicht nur das sprachliche Können eines Fremden herausfordern, sondern auch Fragen in Bezug auf ihren Ursprung stellen. Es gibt aber auch solche, die nicht nur geschichtliche und geografische Erscheinungen, sondern auch charakteristische Eigenschaften des Lebens innerhalb der zu erlernenden Sprachgemeinschaft und die Denkweise ihrer Mitglieder beinhalten.

Eine frei gesprochene Redewendung wird deshalb zum Phraseologischen verformt und verfestigt, weil sie genau die wichtigsten nationalkulturellen Vorbilder anspricht und diese in knapper, aussagekräftiger Form zum Ausdruck bringt. Nicht nur geschichtliche Fakten, Traditionen, Bräuche, wichtige Erscheinungen des gesellschaftlichen, wirtschaftlichen und kulturellen Lebens, sondern auch kulturelle Werte und Orientierungs- und Deutungssysteme dienen als Ausgangspunkt für die Bildung einer Redewendung. Diese Erscheinungen der Realität, aber auch mentale Prozesse und Einstellungen werden im Laufe des Sprachprozesses unterschiedliche, meist kulturspezifische Verfahren der Realitätsverarbeitung durchlaufen. Phraseologismen werden quasi zu sprachlichen kulturellen Stereotypen, welche die für die betreffende Sprachgemeinschaft übliche Mentalität und deren Erfahrungsschatz widerspiegeln (vgl. Pschenkina 1998; Werestschagin und Kostomarov 1983; Földes 2005).

So drückt die Redewendung ,pünktlich wie die Maurer“ eine breite Palette von Wissen über die Kultur aus. Einerseits ist die heutige Bedeutung der Redewendung durchaus posi- 
tiv, was bedeutet, dass Pünktlichkeit auf der Werteskala einen hohen Platz einnimmt, andererseits stellt sie ein Stereotyp über den Beruf der Maurer dar, die dritte Seite liefert der Ursprung der Redewendung: sie bedeutet nicht Pünktlichkeit in Bezug auf den Anfang der Arbeit, sondern dass Maurer auf die Minute genau die Kelle aus der Hand legen, um Feierabend zu machen. Also ist dieses Stereotyp aus der Geschichte heraus negativ gefärbt.

Eine besondere Gruppe von lexikalischen Einheiten, die eine kumulative Funktion erfüllen, bilden die Sprichwörter. Der Hauptunterschied zu den idiomatischen Redewendungen besteht darin, dass Sprichwörter eine vollendete Einheit bilden, meistenteils einen Satz, in dem die Redewendungen eine unvollendete Wortverbindung darstellen. Die Aufgabe von Sprichwörtern ist nicht, irgendwelche Erscheinungen $\mathrm{zu}$ benennen oder $\mathrm{zu}$ beschreiben, ihre Aufgabe ist, die Erscheinungen zu beurteilen. Dabei geht die Beurteilung nicht vom Sprechenden, sondern vom Volk aus. In der Form der Sprichwörter hat das kollektive menschliche Bewusstsein eines Volkes die wichtigsten Anweisungen, Beurteilungen, Einstellungen gespeichert.

„Ein Sprichwort stellt keine einfache Aussage dar. Es drückt die Meinung des Volkes aus. Sie umfasst die Lebenseinstellungen eines Volkes, die Beobachtungen des Volksgeistes. Nicht jede Aussage wird zu einem Sprichwort, nur diejenige, die der Lebensweise und den Gedanken mehrerer Menschen entsprechen, solche Aussagen können Jahrtausende existieren“" (Anikin 1966, S. 3).

Hier sind Beispiele von deutschen Sprichwörtern, die eine Beurteilung, eine klare und eindeutige Botschaft geben, was als gut und schlecht eingeschätzt wird.

Der Mensch lebt nicht vom Brot allein (Priorität des geistigen Lebens).

Dienst ist Dienst (und Schnaps ist Schnaps) (Priorität der Pflichten).

Erst die Arbeit, dann das Vergnügen (Priorität der Pflichten).

Hilf dir selbst, so hilft dir Gott! (Anweisung, aktiv zu handeln)

Habgier ist die Wurzel allen Übels (negative Einschätzung der Habgier).

Neben den Beurteilungen sind implizit Anweisungen zum weiteren Handeln in den Sprichwörtern enthalten. Sie dienen oft auch als Regeln im Rahmen einer nationalen Kultur. Kulturspezifische Kenntnisse werden in der folgenden Tabelle zusammengefasst. 
Tabelle 1: Kulturspezifische Kenntnisse

\begin{tabular}{|c|c|c|}
\hline 1. kulturelle Normen & 2. soziokulturelles Wissen & $\begin{array}{l}\text { 3. kulturspezifisches Wissen } \\
\text { in der Sprache }\end{array}$ \\
\hline 1.1 Tabu & 2.1 Alltagswissen & 3.1 verbale Komponente des \\
\hline 1.2 Gesetze & 2.2. Lebensbedingungen & Grundwissens \\
\hline 1.3 Sitten und Bräuche & 2.3 interpersonale Beziehun- & 3.2 verbale Etikette \\
\hline 1.4 Traditionen & gen & 3.3 rituelle Etikette \\
\hline \multirow[t]{5}{*}{ 1.5 Gewohnheiten } & 2.4 Werte, Überzeugungen, & 3.4 lexikalische Lakunen \\
\hline & Einstellungen & 3.5 Phraseologismen \\
\hline & 2.5 Körpersprache & \\
\hline & 2.6 soziale Konversationen & \\
\hline & 2.7 rituelles Verhalten & \\
\hline
\end{tabular}

\subsubsection{Kulturübergreifende Kenntnisse}

Der Fremdsprachenunterricht bereitet seine Teilnehmer auf die Gegebenheiten der Zielkultur oder auf die Realität des kulturellen Lebens innerhalb der Gemeinschaft vor, welche von der zu erlernenden Sprache bedingt wird. Diese Realität besteht aus einer breiten $\mathrm{Pa}$ lette von unterschiedlichen kulturellen Besonderheiten: Werte, Einstellungen, Traditionen etc.

Die Entwicklung der interkulturellen Kompetenz wird in dieser Arbeit im Kontext des Sprachlernens untersucht, d.h. Sprache und Kultur sind untrennbar verbunden. Was wäre aber, wenn sich ein Mensch auf die Kommunikation mit Vertretern einer anderen Kultur vorbereiten möchte, ohne zu wissen, aus welcher Kultur seine Kommunikationspartner stammen werden? In diesem Fall kann er nicht im Voraus die Besonderheiten einer bestimmten Kulturgemeinschaft erlernen. Das bedeutet aber auch, dass er die Sprache seiner Gesprächspartner wahrscheinlich nicht beherrschen wird. Ein anderer Fall wäre, wenn der Mensch, der sich auf die Kommunikation mit den Vertretern einer Zielkultur vorbereitet hat, trotzdem mit erheblichen Mangelerscheinungen und Lücken in seinem Wissen konfrontiert werden kann.

Gibt es Informationen, die für alle Sprachgemeinschaften und Kulturen relevant sind? Welche interkulturellen Kenntnisse und Fähigkeiten sollten allen Lernenden vermittelt 
werden $?^{23}$

Brislin und Yoshida (1994) beantworten diese Fragen positiv und nennen diese Art von Kenntnissen culture-general. „Such knowledge refers to specific theories or themes that are commonly encountered in cross-cultural interactions regardless of cultures involved. Although these may not provide prescriptive behavior or concrete answers to one's dilemmas, they provide clues as to where the problem(s) may lie" (Brislin und Yoshida 1994, S. 37).

Kulturübergreifende Kenntnisse sind Informationen, welche die Problematik der interkulturellen Kommunikation aufgreifen und den Lernenden dazu verhelfen, sich sogar bei einem Mangel an kulturspezifischen Informationen adäquat an der Kommunikation mit dem interkulturellen Partner zu beteiligen und das Ziel der Kommunikation zu erreichen.

„Adäquates kulturelles Wissen manifestiert sich also darin, dass eine Person auf Grund systematisch analysierter Informationen in der Lage ist, die einzelnen „Regeln“ der fremden Kultur zu einer inneren Logik zusammenzufügen und daraus ein besseres Verständnis für die fremdkulturellen Verhaltensweisen zu entwickeln“ (Meyer und Boness 2004, S. 152).

Kulturübergreifende Kenntnisse sind rein theoretischer Natur. Sie haben als Ziel nicht bloß Wissensaneignung, sondern auch einen weiteren kognitiven Prozess, und zwar Entwicklung des kulturellen Bewusstseins. Mit Hilfe der kulturübergreifenden Kenntnisse wird ermöglicht, aus den punktuellen Kenntnissen über die einzelnen Sachverhalte einer fremden Kultur ein System von Deutungsmustern aufzubauen.

Die Erkenntnis oder das Bewusstsein dieses Wissens stellt für den Sprachlernenden eine weitere Herausforderung, ein weiteres Ziel im kognitiven Bereich dar.

Der Taxonomie der Lernziele (vgl. Bloom 1972, Anderson und Krathwohl 2001) zufolge sollen einige kognitive Prozesse wie Erinnern, Verstehen, Anwenden, Analysieren, Bewerten im Gehirn ablaufen, damit Wissen gebildet werden kann. Ein weiterer Schritt nach der Aneignung von kulturübergreifenden Kenntnissen führt durch die oben erwähnten kognitive Prozesse zu einem bewussten Besitz dieser Kenntnisse. Obwohl das Bewusstsein sich manchmal auch auf verhaltensbezogene und affektive Momente bezieht, ist es trotzdem ein Produkt der mentalen Prozesse, deshalb kann es als weiteres Ziel dem kognitiven Bereich zugezählt werden.

Allein der Ursprung des Wortes „Bewusstsein“ erlaubt es, diese Kategorie im kognitiven

23 Diese Fragen führen zu der Diskussion darüber, ob die interkulturelle Kompetenz „allgemein“ sein kann, d.h. ob es möglich ist, solche Kompetenz zu entwickeln, die den Menschen erlaubt, kompetent in allen Kulturen zu handeln. 
Bereich neben dem Wissen anzusiedeln. Das Wort lässt sich auf das lateinische conscientia

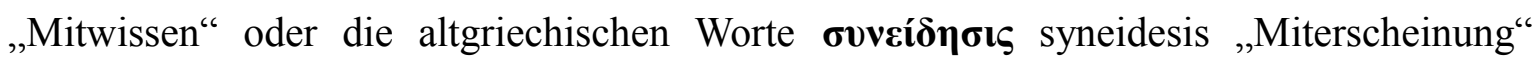

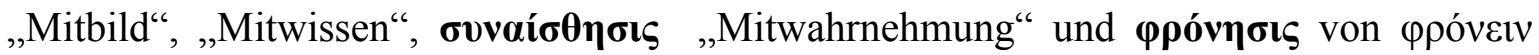
„bei Sinnen sein“, beziehen.

Das Bewusstsein stellt ein umstrittenes und noch kaum erforschtes, in einigen Fällen sogar mysteriöses Konzept dar. Was ist eigentlich „Bewusstsein“? Zurzeit liegt noch keine allgemein anerkannte Definition dieses Begriffes vor. Deshalb wird hier eine aus der Brockhaus-Enzyklopädie angeführt, bevor hier das Bewusstsein im interkulturellen Bereich angesprochen wird.

„Bewusstsein ist die Beziehung des Ich auf einen äußeren oder inneren Gegenstand, das unmittelbare Wissen des Subjekts um geistige oder seelische Zustände, die es erfährt, auch das Aufmerken auf einzelne Erlebnisse“ (Brockhaus 1996, Band 3, S. 267).

Bewusstsein ist nicht bloß Wissen, sondern ein Wissen über Wissen, das in einer direkten und unzertrennlichen Beziehung zur Ich-Komponente unserer Wahrnehmung steht (vgl. Batik 1993)

Das Verhältnis zwischen zwei mentalen Prozessen, und zwar Wissen und Bewusstsein, ähnelt einem Prozess der Filmentwicklung. Die Abbildung, die Kenntnisse im Gedächtnis, sind zwar auf dem Fotonegativ gespeichert, jedoch kaum für jemanden zu erkennen. Das Bewusstmachen dieser Kenntnisse ist wie die Entwicklung des Filmes, sie werden präsent und sichtbar, erkennbar für ihren Träger.

Interkulturelles Bewusstsein ist ein Baustein der interkulturellen Kompetenz, welches von den meisten Theoretikern anerkannt ist (vgl. Brislin 1994; Byram 1997, Fantini 2000). Besondere Aufmerksamkeit wird dem Bewusstsein in der Arbeit von Alvino Fantini zuteil. Seiner Ansicht nach ist das interkulturelle Bewusstsein nicht nur ein abgesondertes Element der interkulturellen Kompetenz, sondern auch gleichzeitig ein Schlüssel für erfolgreiche interkulturelle Kommunikation. Er geht weiter und bezeichnet das Bewusstsein als das wichtigste Element und stellt es in der Mitte seines Modells der interkulturellen Kompetenz. Er geht davon aus, dass Bewusstsein sich immer auf das Verhältnis zwischen „Ich“ und „etwas“ oder ,jemandem“ aus der Außenwelt bezieht und durch Reflexion und Selbstanalyse entsteht.

"Awareness is difficult to reverse; that is, once one becomes aware, it is difficult to return to a state of unawareness (and even though one may try to deceive oneself, the self knows of the deception). Awareness leads to deeper cognition, skills and attitudes just as is also enhanced by their development. It is pivotal to cross-culture entry and to acceptance by 
members of other cultures on their terms" (Fantini 2000, S. 29).

Obwohl Bewusstsein ein Ergebnis eines mentalen Prozesses ist, wird es oft nicht genügen, sich dieses Wissen anzueignen, da Erfahrungen interkultureller Kontakte erforderlich sind. Genauso wenig kann allein interkulturelle Erfahrung ohne ausreichende Kenntnisse zum interkulturellen Bewusstsein beitragen. Eine Person kann die ganze Welt bereist und mehrere Kulturen kennengelernt haben, bleibt aber stur in ihrem Orientierungssystem stecken, wenn ihr Unterschiede in den kulturellen Orientierungen der anderen Nationen nicht bewusst sind.

Das interkulturelle Bewusstsein stellt aber gleichzeitig einen Ausgangspunkt dar. Wenn die Grundlagen des interkulturellen Wissens einer Person bewusst sind, kann sie auch ihre Gefühle bewusst wahrnehmen und steuern, ihre Einstellungen ändern, die in interkulturellen Situationen benötigten Fähigkeiten entwickeln, ihr Verhalten kontrollieren und steuern, also bewusst alle Möglichkeiten nutzen, um die interkulturelle Situation erfolgreich und produktiv zu gestalten. So bezeichnet Geistmann (2002) als zentrale Zielgröße der interkulturellen Kompetenz bewusste Verhaltenssensibilisierung, die er als bewusste Interpretation und bewusstes Gestalten von interkulturellen Interaktionssituationen interpretiert.

Der gemeinsame europäische Referenzrahmen für Fremdsprachen siedelt interkulturelles Bewusstsein auch unter dem Oberbegriff „deklaratives Wissen (savoir)“ an, begleitet von zwei weiteren Bestandteilen: Weltwissen und soziokulturelles Wissen.

Aus der Kenntnis, dem Bewusstsein und dem Verständnis der Beziehungen zwischen der ,Welt des Herkunftslandes` und der ,Welt der Zielsprachengemeinschaft‘ (Ähnlichkeiten und klare Unterschiede) erwächst ein interkulturelles Bewusstsein. Natürlich ist es wichtig festzustellen, dass interkulturelles Bewusstsein auch bedeutet, sich der regionalen und sozialen Verschiedenheit in beiden Welten bewusst zu sein. Ferner wird das interkulturelle Bewusstsein dadurch bereichert, dass es neben den Kulturen, in denen die Muttersprache und die Fremdsprache gesprochen werden, noch viele andere Subkulturen gibt. Dieser weitere Horizont hilft, beide in einen größeren Kontext einzuordnen. Über das objektive Wissen hinaus gehört zum interkulturellen Bewusstsein auch, dass man sich bewusst ist, wie eine Gemeinschaft jeweils aus der Perspektive der anderen erscheint, nämlich häufig in Form nationaler Stereotype.

Kulturübergreifende Kenntnisse stellen einen wichtigen Teil des interkulturellen Wissens dar, ihre Aneignung soll durch Bewusstmachung vervollständigt werden. Sie spielen auch eine wichtige Rolle für die Entwicklung der weiteren Bestandteile der interkulturellen Kompetenz, der interkulturellen Fähigkeiten und der interkulturellen Sensibilität. 
Diese Art von Wissen wird oft in interkulturellen Trainings angeboten, wo die Zielgruppen (meistenteils Manager oder Wirtschaftsleute) auf die Kommunikation mit Vertretern einer anderen Kultur vorbereitet werden. Es ist selten Bestandteil des Fremdsprachenunterrichts, der Lernende wird jedoch auf die Durchführung einer interkulturellen Kommunikation vorbereitet, und es muss damit gerechnet werden, dass er kaum alle Sachverhalte der fremden Kulturwelt im Unterricht erlernen kann. Ein hohes Niveau von Sprachbeherrschung und gute Kenntnisse von landeskundlichen Informationen garantieren dem Sprechenden keineswegs einen reibungslosen Ablauf der Kommunikation, wenn kulturübergreifendes Wissen fehlt.

Kulturübergreifende Kenntnisse sollen parallel zu den anderen sowohl linguistischen als auch extralinguistischen landeskundlichen Kenntnissen im Unterricht vermittelt werden. Da das Sprachniveau in den Lerngruppen unterschiedlich ist, können die kulturübergreifenden Kenntnisse nicht immer mit Hilfe der zu erlernenden Sprache vermittelt werden. Da es oft um theoretische und in Bezug auf den Wortschatz schwierige Sachverhalte geht, kann die Muttersprache dabei verwendet werden. Damit aber kulturübergreifende Kenntnisse nicht ohne Anwendung bleiben, sollen immer Beispiele aus der gegenseitigen Kulturpraxis benutzt werden.

$\mathrm{Zu}$ den kulturübergreifenden Kenntnissen gehören:

- Kenntnisse über die Mehrdeutigkeit des Begriffs Kultur und über unterschiedliche Schichten von Kultur

- Kenntnisse über Kulturtheorien

- Kenntnisse über das Phänomen der Andersartigkeit und seine Beziehung zum kulturellen Selbst

- Kenntnisse über die eigene kulturelle Prägung

- Kenntnisse über nationale Stereotype und ihren Einfluss auf die interkulturelle Kommunikation.

Im Folgenden wird eine kurze Beschreibung der oben angeführten Kenntnisse gegeben.

Kenntnisse über die Mehrdeutigkeit des Begriffs Kultur und über unterschiedliche Schichten von Kultur

Der Begriff „Kultur“ liegt unserer Thematik zugrunde und muss als Erstes auch im Unterricht definiert werden. Reicht aber allein die Definition des Begriffs für das Verständnis des gesamten Komplexes dieser Sachverhalte aus? Genügt es, wenn die Lernenden sich 
diese Definition einprägen? Die Kenntnisse zum Begriff „Kultur“ müssen mehrere Aspekte des Begriffs beinhalten, darunter auch solche aus historischen, psychologischen und sozialen Sichtweisen. Dabei gebührt der „nationalen Kultur“ besondere Aufmerksamkeit; es muss herausgearbeitet werden, dass die Kultur einer Nation, einer Sprachgemeinschaft, eines Volkes, eines Landes oder eines Staates keine einheitliche Erscheinung ist. Das Wissen darüber, dass innerhalb einer (z.B. nationalen) Kultur noch mehrere Subkulturen existieren, die manchmal größere Unterschiede zwischen ihren Vertretern als zwischen den Vertretern der unterschiedlichen (nationalen) Kulturen aufweisen. Am Beispiel der nationalen deutschen Kultur kann gezeigt werden, dass innerhalb dieser durch die Grenzen der BRD vorgegebenen Kulturgemeinschaft unterschiedliche Subkulturen existieren: hinsichtlich der Sprachgemeinschaft und der jeweiligen Region, als da wären bayerische, sächsische, hessische, schwäbische Subkulturen; hinsichtlich sozialer Kategorien städtische, dörfliche, studentische, industrielle Subkulturen; hinsichtlich von Wertvorstellungen traditionelle, moderne, jugendliche, hedonistische Subkulturen etc. Infolge der Globalisierungs- und Zuwanderungsprozesse kommen zu der schon vorhandenen Mehrschichtigkeit der nationalen Kultur noch weitere nationale eingewanderte Kulturgemeinschaften wie türkische, d.h. es muss auch mit türkischen, kurdischen, italienischen, albanischen etc. Subkulturen gerechnet werden.

Diese Erkenntnis zeigt einerseits die vielfache Abhängigkeit der menschlichen Wahrnehmung und des Verhaltens von Einstellungen der eigenen Kultur, andererseits wird das Klischee „Nation - Kultur“ zerstört und ein Verständnis für die Abhängigkeit eines Menschen von unterschiedlichen Subkulturen, denen er sich zugehörig fühlt, entwickelt. Ziel der Vermittlung dieses Wissens ist es, ein Bewusstsein dafür zu schaffen, dass Kultur eine komplexe Erscheinung ist, der jeder Mensch ausgeliefert ist, und dass der Mensch sich als Vertreter von mehreren Subkulturen versteht und verstanden werden soll.

\section{Kenntnisse über Kulturtheorien}

Was bedeutet aber „sich einer Kultur zugehörig fühlen“? Was einen Menschen zum Mitglied einer Kulturgemeinschaft macht und welche Einzelteile sich hinter dem Begriff „Kultur“" verbergen, muss weiter im Themenbereich „Kultur und Kulturtheorien“ besprochen werden. Eines der bekanntesten Modelle ist das Eisbergmodell. Das Modell, das alle Elemente der Kultur in sichtbare und unsichtbare teilt, wobei die sichtbaren Teile die fundamentalen verborgenen Teile zum Ausdruck bringen, verschafft einen Überblick über das, was beim ersten fremden Blick verborgen bleibt. Es gibt auch die Erklärung dafür, wie 
schwierig es sein kann, die realen kulturellen Hintergründe des fremden Wahrnehmens und Handelns zu entdecken.

Die weiteren Theorien versuchen die menschlichen kulturell bedingten Verhaltensmerkmale zusammenzufassen und in eine Gruppe zu kategorisieren. Dabei können die Theorien von Hall und Hall (1989), Hofstede (1993), Thompson (1990) und Rokeach (1973) verwendet werden.

Um nicht jede Theorie im Einzelnen zu diskutieren, soll hier stellvertretend die Theorie von Brislin (1994) vorgestellt werden. Für seine interkulturellen Trainingsmaßnahmen hat er aus allen Theorien eine Auflistung von Themen (Kulturkategorien) erarbeitet, welche für jede Kultur relevant sind. Die von Brislin angesprochenen Kulturkategorien sind: Arbeit, Zeit und Raum, Sprache, Rollenverteilung, Individualismus/Kollektivismus, Rituale und Vorurteile, Hierarchie und Werte.

Bei der Vermittlung dieser Kenntnisse muss gezeigt werden, dass sogar bei den alltäglichsten Angelegenheiten, wie zum Beispiel den Einstellungen zur Zeit oder zur Arbeit, zwischen den Kulturen unterschiedliche Akzentuierungen existieren. An Beispielen aus mehreren Kulturen zeigt Brislin, dass ihre Vertreter solche Konstrukte wie „Zeit“" oder „Arbeit“ auf eigene Weise wahrnehmen und unterschiedlich damit umgehen. Er schlägt vor, die angegebenen Themen in dem interkulturellen Training jeweils auf das Land bezogen, in das der Teilnehmer einreist, anzusprechen. Dies ist sicherlich eine gute Variante bei einem Training, dessen Teilnehmer genau wissen, wohin sie fahren werden, also auf welche Kultur sie sich vorbereiten sollen. Im Fremdsprachenunterricht ist die Zielkultur durch die zu erlernende Sprache bestimmt, deshalb sollten sich die angesprochenen Themen in erster Linie auf die Kultur, deren Sprache erlernt wird, beziehen.

Nicht weniger wichtig ist aber, durch Veranschaulichung der Kulturkategorien das Bewusstsein der Teilnehmer zu schärfen und zu zeigen, dass z.B. in punkto Zeit oder Raum der Vertreter einer anderen Kultur anders denkt und urteilt. Und dafür müssen solche Kategorien, die aus den Kulturtheorien bekannt sind, wie Raum, Zeit, Kontext, Unsicherheitsvermeidung, Machtdistanz im Unterricht angesprochen werden. Rein theoretische Kenntnisse über die Kulturtheorien sollen auf jeden Fall durch praktische Beispiele beleuchtet werden, um zu verdeutlichen, wie die Kategorien in der Zielkultur zum Ausdruck kommen (wie in Brislins Trainingsmaßnahmen). Die Auswahl von Beispielen soll aber nicht durch das Auswahlkriterium „Zielkultur“ eingegrenzt werden. Beispiele aus anderen Kulturen sind unbedingt einzubeziehen. Es hilft den Teilnehmern, sich erstens nicht auf die Unterschiede zwischen der eigenen und der Zielkultur, sondern auch auf die Kategorien selbst zu 
konzentrieren. Zweitens wird m.E. die Bildung nationaler Stereotype durch eine solche Verlagerung der Akzente verringert.

Kenntnisse über das Phänomen der Andersartigkeit und seine Beziehung zum kulturellen Selbst

Der folgenden schematischen Darstellung liegen Kenntnisse über das Phänomen der Andersartigkeit und seine Beziehung zum kulturellen Selbst zugrunde.

Abbildung 6: Das Fremde und das Eigene

Das Eigene, das Fremde, das Interkulturelle

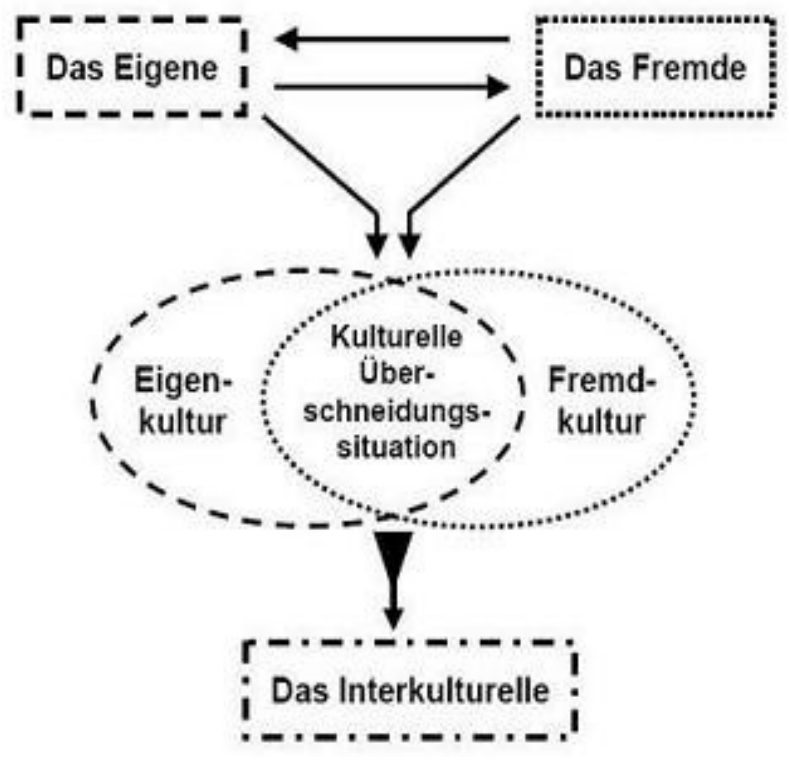

Anhand der aus den Kulturtheorien erarbeiteten Kulturkategorien wird dabei das Wissen vermittelt, dass es sich bei einer interkulturellen Überschneidungssituation um eine Konfrontation zwischen zwei unterschiedlichen kulturellen Welten handelt. Beim Treffen von Menschen aus unterschiedlichen Kulturen prallen zwei oder mehrere kulturelle Hintergründe aufeinander. Jeder Teilnehmer einer solchen Situation interpretiert das Verhalten und die Worte seines Partners auf seine eigene Weise, die nicht nur von den individuellen Einstellungen und der eigenen Geschichte abhängt, sondern vielmehr auf den in der heimischen Kultur angeeigneten Interpretations- und Handlungsmodellen basiert. Dabei hat der Teilnehmer der Überschneidungssituation das Verhalten des Partners zu verstehen, was auch zu inadäquaten Reaktionen führen kann. Wenn man sich jetzt vorstellt, dass beide Gesprächs- 
partner im Laufe der Kommunikation solche Prozesse erleben, ist ein Raum vorstellbar, in dem keine einzelne Kultur ,regiert“.

Das Bewusstsein darüber, dass die Situation des interkulturellen Kontakts immer einen zweiseitigen Charakter hat, ist eine Grundvoraussetzung dafür, dass sich die Teilnehmer darauf einstellen können, dass der Gesprächspartner anders denkt, urteilt und wahrscheinlich auch anders reagieren wird. Solches Bewusstsein liegt auch den Fähigkeiten zugrunde, eine Überschneidungssituation so zu gestalten, dass ein besonderes Klima entsteht, in dem man möglichst nicht auf seine eigene Einschätzungsweise zurückgreift, sondern erst einmal versucht, die Kultur des Partners nachzuvollziehen.

\section{Kenntnisse über die eigene kulturelle Prägung}

Um diese Einstellung zu ermöglichen, müssen Teilnehmer einer Überschneidungssituation bereit sein, eigene kulturelle Hintergründe zu hinterfragen. Dazu sind Kenntnisse über die eigene kulturelle Prägung notwendig. Bei dieser Selbsterkennung geht es im interkulturellen Fall nicht in erster Linie um persönliche, sondern um kulturell geprägte Eigenschaften, Lebensweisen und Verhaltensmerkmale.

Es sind dies Verhaltensweisen und Einstellungen, Weltbilder und Menschenbilder, die sie mit Menschen teilen, die in gleichen kulturellen Kontexten aufgewachsen sind und von ihnen geprägt wurden (vgl. Flechsig 2001, S. 106).

Diese Einstellungen scheinen dem Menschen selbstverständlich zu sein, die dazugehörigen Verhaltensweisen nimmt er unbewusst als normal wahr. Diese Einstellungen und Verhaltensweisen werden nur in der Konfrontation mit dem „Fremden“ spür- und sichtbar, deshalb werden sie dann in Frage gestellt.

Damit der Sprachlernende mit dem Problem der kulturellen Selbsterkennung nicht das erste Mal bei einem interkulturellen Kontakt konfrontiert wird, sollen Kenntnisse über kulturelle Prägungen, vermittelt werden, auf deren Grundlage die Fähigkeit zur Selbstreflexion entwickelt werden kann. Diese Kenntnisse beinhalten nicht nur das Wissen über solche Begriffe wie Ethnozentrismus, kulturelle Identität, Enkulturation, Selbstbild, sondern auch die Kenntnisse darüber, was der Mensch aus kultureller Sicht darstellt. Die Vermittlung der Erkenntnis der eigenen kulturellen Prägung soll zum Bewusstsein der eigenen kulturell-ethnozentrischen Position führen. Ergänzend zu der Vermittlung der kulturellen Theorien sollen die kulturellen Orientierungen in den Fokus gerückt und bewusst wahrgenommen werden. Genauso bewusst soll auch die Grenze des „Eigenen“ in der interkulturellen Überschneidungssituation anerkannt werden. 
Kenntnisse über nationale Stereotype und ihren Einfluss auf die interkulturelle Kommunikation.

Eine Gefahr für interkultureller Kontakte stellt aber das schon durch die Enkulturation angeeignete Wissen über andere Nationen dar. Es existiert in Form nationaler Stereotype oder von Fremd- und Selbstbildern und stellt sich verallgemeinert in den in der heimischen Kultur üblichen Vorstellungen über Vertreter sowohl der fremden als auch der eigenen Kultur dar. Um den Einfluss dieser verzerrten Bilder zu minimieren, sollen Kenntnisse über nationale Stereotype und ihren Einfluss auf die interkulturelle Kommunikation vermittelt werden. Diese Kenntnisse sollten jedoch von den Teilnehmern selbst gewonnen werden. Als Grundlage können einige Informationen über die Natur der schematischen (stereotypischen) Wahrnehmungen und speziell über die Natur der nationalen Stereotype mitgeteilt werden.

Die Erkenntnis darüber, welche schematisierten Bilder von den eigenen und fremden Nationen in jedem einzelnen Kopf und auch im Gruppengedächtnis gespeichert sind, d.h. das Preisgeben der eigenen nationalen Stereotype, ist ein Zwischenziel der Vermittlung dieser Kenntnisse. Aber auch das Bewusstsein darüber, dass ein schematisiertes Denken und das Vorhandensein der nationalen Stereotype in der eigenen Wahrnehmung völlig normal sind, soll von den Sprachlernenden erkannt werden. Stereotype an sich sind fast unausweichlich, sie wirken aber negativ auf die interkulturelle Kommunikation, wenn sie nicht reflektiert werden.

Die Suche nach möglichen historischen Hintergründen der existierenden Stereotype oder die Gewinnung von Wissen über die stereotypischen Bilder, die im Gruppengedächtnis einer anderen Nation in Bezug auf die eigene existieren (reziprokes Selbstbild), sind weitere mögliche Schritte in diesem Wissensbereich. Es ist aber auch wichtig, am Beispiel einer oder mehrerer interkultureller Überschneidungssituationen $\mathrm{zu}$ fokussieren, welche negativen Auswirkungen der Gebrauch von unreflektierten Fremdbildern auf den Prozess der gegenseitigen Verständigung haben kann. Das Wissen darüber, warum ein interkulturelles Missverständnis aufgetreten ist, soll von den Lernenden selbst im Laufe der Analyse gewonnen werden.

Zusammenfassend kann gesagt werden, dass die erste Komponente des in dieser Arbeit behandelten Lernmodells der interkulturellen Kompetenz das interkulturelle Wissen darstellt. Diese Komponente präsentiert die kognitive Seite des Modells, die kognitiven Lernziele im Rahmen des Fremdsprachenunterrichts, deren Richtziel „,interkulturelle Kompetenz“ ist. 
Dieses Wissen besteht aus zwei Arten von Kenntnissen: kulturspezifischen und kulturübergreifenden Kenntnissen. Kulturspezifisches Wissen stellt eine breite Palette aus unterschiedlichen soziokulturellen Kenntnissen dar. Einige Komponenten wie die Kenntnisse über das alltägliche Leben, über Lebensbedingungen, interpersonale Beziehungen, Körpersprache, soziale Konversationen oder rituelles Verhalten sind im Laufe der interkulturellen Kommunikation erkennbar und deshalb relativ leicht zu vermitteln. Kulturelle Normen wie Tabus, Gesetze, Sitten und Gebräuche, Traditionen, Bräuche und Gewohnheiten bleiben dem ersten fremden Blick verborgen und lassen sich deshalb schwerer vermitteln. Die nächste Komponente der kulturspezifischen Kenntnissen ist das kulturspezifische Wissen in der Sprache, welches sich durch Grundwissen, Lakunen, phraseologische Redewendungen und Aphorismen ausdrückt. Die kulturübergreifenden Kenntnisse schließen die Kenntnisse über Mehrdimensionalität der Kultur, Kulturtheorien, Phänomen der Andersartigkeit, eigene kulturelle Prägung und nationale Stereotype mit ein. Interkulturelles Bewusstsein über erlernte Sachverhalte spielt innerhalb dieser Komponente eine große Rolle.

\subsection{Die interkulturelle Sensibilität als affektive Komponente}

\subsubsection{Emotionen im Unterricht}

Das Puzzlemodell der interkulturellen Kompetenz beschreibt drei Seiten des Unterrichtsprozesses: kognitive, affektive und pragmatische. Im vorigen Kapitel wurde diskutiert, wie die Entwicklung der kognitiven Seite gefördert werden kann, und zwar welche Kenntnisse im Unterricht angeeignet und bewusst gemacht werden müssen. Sobald aber die Person des Lernenden engagiert ist, sind Emotionen im Spiel. Sie spielen eine entscheidende Rolle für die weiteren Einstellungen des Lernenden zum Unterricht und dürfen deshalb nicht unbeachtet und unangesprochen bleiben. Deshalb ist der Umgang mit den Emotionen ein wichtiger Teil des Unterrichtsprozesses. Die affektive Seite nimmt auch einen wichtigen Platz im Puzzlemodell ein.

„Die übermäßige Akzentuierung der Kognition und deren Abtrennung vom Affekt bedeutet eine Bedrohung unserer Gesellschaft, denn unsere Erziehungsinstitutionen könnten kalte einzeln stehende Individuen hervorbringen, die sich humanitären Zielen nicht verpflichtet fühlen ... Wir können z.B. genau wissen, welche Ungerechtigkeiten Minderheiten in unserer Gesellschaft erfahren, solange wir aber davon nicht auch gefühlsmäßig angesprochen 
werden, dürften wir kaum etwas dagegen tun. Solange Kenntnisse nicht zum affektiven Erleben des Lernenden in Beziehung gesetzt werden, ist die Wahrscheinlichkeit begrenzt, dass sie Einfluss auf dessen Verhalten nehmen werden“ (Weinstein und Fantini 1974, zitiert aus Mietzel 2007, S. 438).

Die affektive Seite eines Lernprozesses wurde in der Vergangenheit geringer eingeschätzt als seine kognitive Seite. Dank der Autoren der humanitären Richtung wurde die Bedeutung von Emotionen und Einstellungen im Unterricht erkannt: die Lehrer wurden aufgefordert, eine harmonische Zusammenentwicklung der Kognition und des Affekts zu erzielen. Aus dem oben angeführten Zitat von Weinstein und Fantini ist die Bedeutung der affektiven Seite für die interkulturelle Kompetenz deutlich erkennbar. Die Gefühle und Einstellungen, die der Lernende bezüglich des Lerninhalts empfindet, wirken entscheidend auf die Entwicklung seiner Fähigkeiten und Fertigkeiten im Unterrichtsprozess und auf sein weiteres Verhalten. Stellen wir uns folgende Situation vor: Der Lernende soll eine Fremdsprache in der Schule oder Hochschule lernen ${ }^{24}$, er hat aber eine negative Einstellung oder sogar eine Abneigung zu dem Land oder der Kultur. Dies wird sich auf seine Lernmotivation auswirken und den Lernerfolg beeinträchtigen. Das Gleiche gilt auch für die interkulturelle Kommunikation. Wie soll denn die Kommunikation verlaufen, wenn einer von zwei Partnern negativ zu dem anderen eingestellt ist und/oder sich nur auf seine eigenen Vorurteile stützt?

Die affektive Seite des Unterrichts hat die Entwicklung von Einstellungen, Werten und Emotionen zum Inhalt. Aber gerade diese Inhalte sind relativ schwer zu operationalisieren. Während sich die Kenntnisse, die im Unterricht zu erlernen sind, relativ gut bewerten lassen, sind die affektiven Komponenten schwer einzuschätzen. Eine weitere Schwierigkeit besteht darin, dass es manchmal Jahre oder sogar Jahrzehnte dauern kann, bis eine bestimmte affektive Komponente ausgebildet wird. Das betrifft besonders solche Inhalte wie Werte und Einstellungen.

Das affektive Lernen wird nicht als Aneignung fremder Werte und Einstellungen verstanden. Es geht also nicht um eine Art von Enkulturation, wie z.B. in der Theorie von Werestschagen und Kostomarov, die diesen Prozess als Akkulturation bezeichnen.

Die Verfasserin versteht unter der affektiven Komponente der interkulturellen Kompetenz nicht die in der jeweiligen Kultur existierenden Werte und Einstellungen, sondern solche, die zum Erfolg der interkulturellen Kommunikation beitragen.

24 Im Russland ist eine Fremdsprache in jeder Schule oder Hochschule ein Pflichtfach. Die Auswahl einer Fremdsprache soll der Lernende meistens im 4. Schuljahr treffen. 
Aus den oben erwähnten Gründen wurde die affektive Seite der interkulturellen Kompetenz in das Puzzle-Modell aufgenommen. Obwohl Einstellungen als selbstständige Komponente darin nicht vorkommen, sind sie als fernes Ziel gemeint. Ein Fremdsprachenunterricht setzt in Bezug auf die Zeit und in Bezug auf die Möglichkeit, die erlernte Sprache in der Praxis zu benutzen, einen relativ begrenzten Rahmen. Denn es ist zweifelhaft, ob innerhalb dieses Raumes die Einstellungen der Lernenden geändert werden können. Deshalb wird als Ziel die Entwicklung der interkulturellen Sensibilität formuliert, sie soll im Weiteren dazu beitragen, die Einstellungen und Haltungen des Lernenden im Unterricht und auch im Alltag der interkulturellen Kommunikation zu verändern.

Interkulturelle Sensibilität ist ein mehrdeutiger Begriff. Aus psychologischer Sicht bedeutet Sensibilität „,das Vermögen, auf äußere Reize mit seelischen Vorgängen zu reagieren. (Gefühlsansprechbarkeit, Empfindsamkeit, Feinfühligkeit)“(Brockhaus 1996, Band 20, S. 70).

Emotionale Sensibilität ist ein wichtiger Bestandteil der sozialen Kompetenz oder der emotionalen Intelligenz und wird häufig als eine wichtige Voraussetzung für die Bewerbungsprofile des modernen Fachmannes oder der Fachfrau erwähnt. Damit ist ein Gespür für die Stimmungen anderer Menschen gemeint, das sich in der Aufmerksamkeit gegenüber versteckten Signalen z.B. in der Körperhaltung, Mimik und Lautstärke äußert.

Interkulturelle Sensibilität zeigt sich im Gespür gegenüber den Besonderheiten einer interkulturellen Kommunikation, das dem Menschen erlaubt, sich auf die Andersartigkeit des Gesprächspartners einzulassen. Dank der interkulturellen Sensibilität ist es möglich, die Häufigkeit von Missverständnissen, deren Ursache unterschiedliche kulturelle Hintergründe der Gesprächspartner sind, zu reduzieren. Bei fehlgeschlagener interkultureller Kommunikation hilft die Sensibilität die Gründe dafür zu verstehen oder das Gespräch sogar wiederherzustellen.

Die emotionale Sensibilität wird im Alltag mit dem Einfühlungsvermögen gleichgesetzt. Das stimmt nur innerhalb eines kulturellen Kreises und kann nicht ohne Weiteres auf die Situation eines zwischenkulturellen Kontaktes bezogen werden. Mit Einfühlungsvermögen ist eine Fähigkeit gemeint, sich in die Gefühle und Sichtweisen anderer Menschen hineinversetzen zu können. Es ist aber sehr problematisch, sogar manchmal unmöglich, wenn ein anderer Mensch anders denkt oder ein anderes Wertesystem hat. Der übliche Versuch, sich auf eigene Gefühle und Reaktionen zu stützen, um das Verhalten des Partners zu verstehen, ist häufig zum Scheitern verurteilt.

Aus diesem Grund wird im Fremdsprachenunterricht nicht nur emotionale Sensibilität als Ziel formuliert, sondern die Herausbildung interkultureller Sensibilität angestrebt. Die Ler- 
nenden werden für die Umstände sensibilisiert, die einen zwischenkulturellen Kontakt zu einer besonderen Art von Kommunikation machen und ihren fremdkulturellen Partner als einen besonderen Gesprächspartner, der oft anders denkt und urteilt, der aber mit besonderer Aufmerksamkeit und aufrichtigem Interesse behandelt werden muss, erleben.

Wenn Sensibilität als affektives Ziel angestrebt wird, wird die allgemeine Sensibilisierung als Prozess intendiert. Die Inhalte der emotionalen Seite des Unterrichtsprozesses sind bis heute nicht eindeutig definiert. Herbrandt (2000) stellt in den Mittelpunkt kulturelle Unterschiede im Denken und Handeln, aus denen sich die weiteren sensibilisierungsbedürftigen Felder wie Problem- und Konfliktpotenziale in der interkulturellen Interaktion oder Synergiepotenziale der interkulturellen Arbeit ableiten lassen. Sensibilisierung selbst betrachtet er als Fähigkeit „relevante Reize und Signale aus der Umwelt wahrzunehmen“ (Herbrandt 2000, S. 90). Die interkulturelle Sensibilität soll „eine weltoffene unvoreingenommene Wahrnehmung kultureller Unterschiede und damit ein vorurteilfreies und tolerantes Herangehen an fremde Kulturen ermöglichen“ (Herbrandt 2000, S. 90). Als Begleitprozess der Sensibilisierung erwähnt er die Verbesserung der Empathie und idealerweise Steigerung des Interesses an der fremden Kultur und an deren Angehörigen.

Um die affektive Seite der interkulturellen Kompetenz zu entwickeln schlägt Brislin vor, an Emotionen gegen Ethnozentrismus zu arbeiten. Die Gründe für die von Herbrand angesprochenen Problem- und Konfliktpotenziale sieht Brislin in der durch die Sozialisation angeeigneten ethnozentrischen Einstellung, die als eng mit dem Prozess des Selbstverständnisses verbundene Denkweise definiert wird. Im Prozess der Sozialisation, die gleichzeitig eine Enkulturation ist, lernen die Kinder, was ,gut“ und was „,schlecht“ ist. So lernen amerikanische Kinder, dass eine eigene Meinung zu haben wichtig ist. Seine eigene Meinung zugunsten jemandem anderem zu ändern ist „heuchlerisch“, also „falsch“ und „schlecht“. Wenn solch ein Kind (oder später ein Erwachsener) in eine andere, z.B. japanische Kultur gerät, wo für jedes Gesellschaftsmitglied parallel zwei „Wahrheiten“ existieren, und zwar die eigene und die öffentliche Meinung, ist seine Reaktion unvorhersehbar. Die Palette seiner Reaktionen kann von Enttäuschung, der die Beurteilung: ,ein schlechten Japaner" folgt, bis zur Verwirrung reichen. Deshalb schlägt Brislin vor, mit den Emotionen schon während des interkulturellen Trainings zu arbeiten (vgl. Brislin und Yoshida 1994). Antor entscheidet sich für den Begriff „konstruktivistische Sensibilität“ und richtet seinen Sensibilisierungsprozess gegen die vereinheitlichenden und verzerrten Bilder der Wirklichkeit, also gegen Stereotype und nationale Schemata. Die konstruktivistische Sensibilität soll dazu beitragen, solche Bilder, die sich als Fakten darstellen, nicht ,,als ontologisch Ge- 
gebenes“ zu verstehen, sondern darin „den Konstruktcharakter beispielsweise der Bilder der nationalen Identität zu erkennen“ (Antor 2002, S. 145). Es ist zu erwähnen, dass die affektive Dimension der interkulturellen Kompetenz von Antor nicht auf die konstruktive Sensibilität begrenzt ist. Er spricht auch über weitere drei Komponenten, die helfen, eine affektive Disposition zu schaffen. $\mathrm{Zu}$ einem ist das die Verhinderung der reflexartigen $\mathrm{Ab}$ lehnung des fremden Anderen, zum anderen Offenheit und Toleranzbereitschaft. Als weitere Bedingung wird die Bereitschaft genannt, die Begrenztheit der eigenen Position zu akzeptieren.

Wichtig beim Verständnis der interkulturellen Sensibilität ist die Bereitschaft des Lernenden, auf die Bedürfnisse des Gesprächspartners einzugehen. So definiert Reisch (1995) Sensibilität als ,die Haltung, sich für die Befindlichkeit von Mitmenschen zu interessieren und diese in eigenen Aktionen zu berücksichtigen“ (zitiert nach Geistmann 2002 S. 33-38). Meyer (2004) schränkt die interkulturelle Sensibilität auf „Motivation des Partners, die kulturellen Unterschiede in der Kooperation wahrzunehmen, zu akzeptieren und wertzuschätzen“, ein (Meyer 2004, S. 155). D.h. der Sensibilisierung gegenüber den kulturellen Unterschieden liegt noch die Bereitschaft zugrunde, sich in diesen Prozess zu begeben. Im Weiteren umfasst die interkulturelle Sensibilität laut Meyer drei Eigenschaften: die Aufgeschlossenheit gegenüber fremdkulturellen Denk- und Verhaltensweisen, die Unvoreingenommenheit als Akzeptanz kultureller Unterschiede und das kulturelle Synergiestreben, die kulturellen Unterschiede für den Erfolg der Zusammenarbeit auszunutzen.

Die oben erwähnten Studien ermöglichen einen Einblick in die Diskussion bezüglich der affektiven Dimension der interkulturellen Kompetenz, insbesondere bezüglich der Frage, was unter dem Begriff der interkulturellen Sensibilität verstanden wird. Die interkulturelle Sensibilität stellt einen komplexen Sachverhalt dar, der sich aus bestimmten Haltungen, Einstellungen und Fähigkeiten zusammensetzt. Die wichtigsten sind:

- eine weltoffene Haltung, die ermöglicht, kulturelle Unterschiede wahrzunehmen;

- ein ehrliches Interesse an dem Gesprächspartner und seiner Heimatkultur;

- die Fähigkeit, mit den eigenen Gefühlen wie Angst, Enttäuschung, Verwirrung etc. umgehen zu können;

- ein Gespür, das ein Verhalten ohne Zugriff auf Vorurteile und Stereotype erlaubt;

- die Fähigkeit, auf die eigene ethnozentrische Position bei dem interkulturellen Kontakt zu verzichten;

- die Bereitschaft, auf das Interesse, die Bedürfnisse und sogar auf den emotionalen 
Zustand des interkulturellen Gesprächspartners einzugehen;

- die Motivation, die Ziele der Kommunikation sowohl bei der zwischenmenschlichen Verständigung als auch bei der Lösung gemeinsamer Aufgaben im Betrieb zu erreichen.

Da die interkulturelle Sensibilität so komplex ist und sich nicht als eine einzige Fähigkeit oder eine Einstellung definieren lässt, entscheidet sich die Verfasserin, sie zu definieren als „Gespür“ dafür, „die Besonderheiten sowohl des Denkens, Fühlens und Handelns des fremdkulturellen Partners als auch der kommunikativen Situation selbst wahrzunehmen und zu akzeptieren, und davon abhängig sein Verhalten und seine Gefühle zu steuern“. ${ }^{25}$

\subsubsection{Werte und Einstellungen}

Die interkulturelle Sensibilität bezieht sich auf den affektiven Bereich der Situation eines interkulturellen Kontakts, der Grad ihrer Entwicklung hängt aber von den allgemeinen Einstellungen eines Menschen, seinen sozialen und individuellen Kompetenzen ab. Wenn ein Mensch bestimmte Einstellungen und Eigenschaften besitzt, die ihn bei der Kommunikation mit einem Vertreter des eigenen kulturellen Kreises weiterbringen, ist er sozusagen auch für eine interkulturelle Kommunikation „,besser ausgestattet“. Die interkulturelle Sensibilität besteht quasi aus den in diesem Kapitel beschriebenen Einstellungen und emotionalen Fähigkeiten, die innerhalb der interkulturellen Thematik eine neue Schattierung, einen neuen Klang bekommen.

Unter Empathie meint der US-amerikanischen Psychologie und Psychotherapeut Carl Rogers „das einfühlende Verstehen, ein sich in die innere Welt eines Anderen hineinversetzen“ (Gröning 2006, S. 89). Diese Fähigkeit wird dann aktualisiert, wenn bewusst oder unbewusst etwas nicht offen ausgesprochen wird. Wie schon mehrmals erwähnt, fehlt für beide Kommunikationspartner in einer interkulturellen Situation ein gemeinsames Grundwissen. Was der eine als selbstverständlich wahrnimmt und deshalb keine Notwendigkeit sieht, es anzusprechen, bleibt für den anderen verborgen und deshalb unklar. Empathie hilft, solche Situationen zu vermeiden. Wenn aber die Situation schon fehl-

25 Das Wort „Gespür“ soll in unserem Fall keine mythische Schattierung haben. Gespür bedeutet, etwas Unklares besser erkennen zu können. Die Situation eines interkulturellen Kontaktes verbirgt so viel Unsichtbares und Unbekanntes für ihre Teilnehmer, dass Wissen und sehr gute kommunikative Fähigkeiten allein dabei nicht ausreichen, um die Situation zu verstehen. Dazu ist auch das Gespür notwendig. Ins Russische werden beide Wörter „Gefühl“ und „Gespür“ mit dem Wort „чувство“ übersetzt. 
geschlagen ist oder fehlzuschlagen droht, kann ein empathischer Mensch versuchen, sich in die Position seines Kommunikationspartners zu versetzen, seine Gedanken und Gefühle dabei zu verstehen, und aufgrund dessen sein weiteres Verhalten zu koordinieren. Dabei besteht aber die schon erwähnte Gefahr, die Gefühle und die Gedanken des anderen auf eigene kulturelle Weise zu interpretieren, was nicht immer zutreffend sein wird.

„Um sich aufeinander einstellen zu können, müssen die Kommunikationspartner die Situation jeweils aus der Sicht des anderen betrachten, um auf diese Weise die zu erwartenden Reaktionen des Gegenübers vorweg zu erkennen und im eigenen kommunikativen Handeln zu berücksichtigen“ (Schaub und Zenke 1997, S. 116).

Es passiert relativ oft, dass die Anekdoten, die einem fremdkulturellen Gesprächspartner erzählt werden, nicht die erwartete Lachreaktion auslösen. Es liegt nicht immer am mangelhaften Humorsinn oder mangelnden Sprachkenntnissen, sondern häufig an dem fehlenden Hintergrundwissen. Eine empathische Haltung in diesem Fall wäre, das notwendige Hintergrundwissen zu sichern, bevor die Anekdote erzählt wird.

Empathie schließt die Bereitschaft ein, die eigenen kulturellen Normen zu relativieren. Dieser Bereitschaft geht das Bewusstsein der eigenen kulturellen Prägung voraus. Die Erkenntnis, dass die Welt in der eigenen Wahrnehmung schon durch die eigenkulturellen Erfahrungen und auch durch die nationalen Vorurteile gefärbt ist, ruft nicht immer positive Emotionen hervor. Mit dieser Erkenntnis werden die Probleme dort aufgedeckt, wo sie früher nicht existierten, was manchmal zu Widerständen führen kann. Hier ein Beispiel: Während einer Vortragsreihe an der Universität Göttingen begrüßte eine Referentin das Publikum mit einer afrikanischen Geste, was Verlegenheit, Unzufriedenheit oder sogar Empörung bei einigen Zuhörern auslöste. Dabei war das Thema des Referats unmittelbar der interkulturellen Arbeit gewidmet. Dieses Beispiel zeigt, dass die Zuhörer sich durch die Verwendung einer fremden Geste in dem Raum, der als „eigenes Territorium“ gilt, persönlich betroffen fühlten. Trotz des angesprochenen Themas waren sie nicht bereit, eigene Normen in Frage zu stellen. Die interkulturelle Sensibilität schließt unter anderem diese Bereitschaft ein, die eigene kulturelle Prägung zu erkennen, in die Akzeptanz des Fremden aber eigene kulturelle Einstellungen einzubeziehen und infolgedessen die eigenen kulturellen Normen zu relativieren. Über die Bedeutung solcher Bereitschaft im Fremdsprachenunterricht schreibt Tolkiehn:

„Die Konfrontation mit dem Eigenen als Gegenstand der Betrachtung und des Hinterfragens kann allerdings aus schon besprochenen Gründen zu Widerständen führen. (...) Es ist jedoch wichtig, sich über solche Widerstände im FU hinwegzusetzen, um eine größere Ak- 
zeptanz des Fremden im Eigenen und folglich des Anderen zu ermöglichen und somit eine zusätzliche Dimension der Verständigung zu erschließen“ (Tolkiehn, S. 298).

Eine Grundlage für die Bereitschaft, die eigenen kulturellen Normen zu relativieren, bildet Offenheit gegenüber den äußeren Einflüssen. Offenheit oder Unvoreingenommenheit gegenüber Menschen aus anderen kulturellen Kreisen im Zusammenspiel mit aufrichtigem Respekt vor diesen Menschen ist eine notwendige Voraussetzung für den Erfolg eines interkulturellen Kontakts. Bei dieser Haltung ist es enorm wichtig, dass Respekt und Offenheit nicht gespielt sind, sondern die Widerspiegelung der echten Gefühle darstellen.

Offenheit und Respekt sind keine interkulturell spezifischen Werte. Sie sind bei jedem Mitglied jeder menschlichen Gemeinschaft erwünscht und willkommen, da sie den Menschen die Kommunikation und das Erfüllen einer gemeinsamen Aufgabe erleichtern. In der Situation eines interkulturellen Kontakts, die durch negative Gefühle oft erschwert ist, gewinnen Offenheit und Respekt besondere Bedeutung. Die Konfrontation mit den fremden Verhaltensweisen, Ideen und Werten wird häufig als Bedrohung für die eigene Welt empfunden. Gefühle wie Angst, Misstrauen und Wut sind mögliche Reaktionen. Vielerlei Beispiele dafür sehen wir in unserem modernen Alltag, zum Beispiel bei den Begegnungen von Migranten und Einheimischen. Wenn aber in den Grundeinstellungen eines Menschen Offenheit einen bedeutsamen Platz einnimmt, ist ein Schritt in Richtung positiver Gefühle gemacht. Unter dieser Voraussetzung stehen die negativen Gefühle nicht im Vordergrund, und die interkulturelle Konfrontation wird als eine Chance betrachtet, „sich neue Perspektiven zu erschließen, von denen aus bestehende Prozesse hinterfragt und alternative Lösungen erkannt werden können“ (Meyer 2004, S. 155).

Offen sein ist im interkulturellen Kontext eine aktive Position. Offen sein heißt nicht nur Aufgeschlossenheit für die externen Einflüsse, es heißt auch Interesse und Neugier auf Fremdes. Auf das Fremde einzugehen, sich für die Gründe des fremden Verhaltens zu interessieren, die anderen Werte wahrzunehmen und zu respektieren, sind Zeichen von Offenheit. Auf deren Grundlage entwickelt sich die Motivation zu einer gemeinsamen Kommunikation und die Bereitschaft, die aufzutretenden Probleme gemeinsam und akzeptabel für beide Seiten zu lösen.

Probleme einer multikulturellen Gesellschaft sind häufig in der mangelnden Offenheit und dem Fehlen von Respekt gegenüber dem Fremden verankert. Das zeigt sich in der folgenden Meinung eines Einheimischen: „Ich habe nichts gegen Ausländer. Ich mache nichts Schlechtes gegen sie. Ich nehme an keiner Bewegung teil, die für die Vertreibung der ausländischen Bürger ist. Ich störe sie nicht, ich möchte aber auch nicht gestört werden“. Die- 
se Position mag tolerant sein, es mangelt ihr aber eindeutig an Offenheit. Es ist keine gute Grundlage für gemeinsame Tätigkeit, sei es Arbeit, Kommunikation oder einfaches Zusammenleben.

Ambiguitätstoleranz ist eine weitere Komponente der interkulturellen Sensibilität.

Bei einem interkulturellen Kontakt, wenn der fremdkulturelle Kommunikationspartner völlig unterschiedliche Interpretations-, Kommunikations- und Verhaltensweisen zeigt, sind Verunsicherung und Zweifel natürliche Reaktionen. Sie werden dadurch erzeugt, dass die beiden Kommunikanten vor dem Problem stehen, das Verhalten und die Denkweise des anderen nicht nachvollziehen und deshalb nicht prognostizieren zu können. Sie wissen oft nicht, wie sie sich verhalten sollen, welches Verhalten in der vorliegenden Situation üblich ist. Der Wissensstand über die Kultur des Kommunikationspartners reicht nicht aus; es ist einfach nicht möglich, das übliche Verhalten in jeder möglichen Situation des interkulturellen Kontakts zu lehren. Gerade in diesem Moment ist Ambiguitätstoleranz erforderlich.

Ambiguitätstoleranz (lat. ambiguous schwankend, ungewiss, zweideutig, tolerare ertragen, dulden; engl. ambiguity tolerance) beschreibt die Fähigkeit, mit unstrukturierten und widersprüchlichen Situationen umgehen zu können, d.h. sie aushalten zu können und dabei handlungsfähig zu bleiben (vgl. Cockwell 2010, S. 34).

Im interkulturellen Kontext heißt es, die Uneindeutigkeiten, Ungewissheiten der Situation des interkulturellen Kontaktes ertragen und die Lösungen, der durch diese Situationen erzeugten Probleme finden zu können. Ein Mangel an Ambiguitätstoleranz führt dazu, dass die Kommunikationspartner die Situation als unkomfortabel und sogar bedrohlich wahrnehmen, sie zeigen sich desinteressiert und verängstigt. Personen mit einem hohen Grad an Ambiguitätstoleranz bleiben dagegen positiv eingestimmt und interessiert, sie suchen nach Kompromissen und versuchen, einfache und klare Lösungen zu den Problemen zu finden. Zwei Bedingungen sind für die Entwicklung der Ambiguitätstoleranz wichtig: eine starke Identität und gute Beherrschung der Problemlösungsstrategien. ${ }^{26}$ Eine starke Selbstbewusstheit und ein gut entwickeltes Selbstwertgefühl geben dem Kommunikationspartner in solchen gespaltenen Situationen Halt und Kraft. „Eine hohe Ambiguitätstoleranz gilt im Allgemeinen als Zeichen seelischer Ausgeglichenheit und Ichstärke“ (Schraub und Zenke 1997, S. 22). Andererseits ist eine aktive Position bezüglich der auftretenden Probleme, Aufgeschlossenheit, Bereitschaft und Mut, sie zu lösen, eine Vorbedingung.

Ein Beispiel von mangelnder Ambiguitätstoleranz wäre die Geschichte einer amerikani-

26 Quasi spiegelt die Ambiquitätstoleranz die grundlegende Beziehung des Interkulturellen: Ich und die Andere, einerseits wie ich mich selbst wahrnehme und andererseits wie ich mit dem Fremden umgehe. 
schen Studentin japanischer Herkunft. Mit Hilfe eines Austauschprogramms wurde eine längst ersehnte Reise nach Japan, ins Land ihrer Vorfahren für sie möglich. Die erste Enttäuschung erlebte sie schon bei der Ankunft im Flughafen, als sie die Sprache der sie umgebenden Menschen fast nicht verstand, obwohl sie die japanische Sprache von ihren Eltern gelernt und durch Kurse an der Universität vertieft hat. Andere Enttäuschungen und Missverständnisse folgten den ersten. Nach einer kurzen Zeit wollte die frustrierte Studentin nur noch nach Amerika zurück. Die Situation für diese Studentin war sicherlich nicht einfach. Die Kluft zwischen den Erwartungen und Hoffnungen, in der ,alten Heimat“ zurück zu sein, deren Traditionen und Denkweisen verstehen zu können, und der Realität, wo sowohl die Kommunikationsmittel als auch das Verhalten und Denken der Einheimischen fremd erscheinen, war groß. Um diese Zweideutigkeit ertragen zu können und aus dieser schwierigen Situation das sicherlich vorhandene positive Potenzial herauszuziehen, wurde Ambiguitätstoleranz benötigt.

Interkulturelle Sensibilität stellt eine affektive Komponente der interkulturellen Kompetenz dar und setzt sich aus unterschiedlichen Eigenschaften, Werten, Einstellungen und Fähigkeiten einer Person zusammen. Besonders wichtig für die Entwicklung der Sensibilität sind Empathie, die Bereitschaft, die eigenen kulturellen Normen zu relativieren, Offenheit und Respekt im Umgang mit fremden Kulturen und Ambiguitätstoleranz.

\subsubsection{Entwicklungsmodelle zur interkulturellen Sensibilität}

Die Entwicklung der interkulturellen Sensibilität bedeutet die Veränderung von Einstellungen (vgl. Bennett 1993, Gehring 2002), was sicherlich eine der schwierigsten Lernziele darstellt. Die Gründe dazu sind unterschiedlicher Natur. Die Veränderung von Einstellungen ist ein Vorgang, der viel Zeit benötigt, im Vergleich z.B. mit der Entwicklung einiger Fähigkeiten oder der Aneignung von Kenntnissen. Außerdem ist das Resultat schwer unter den künstlichen Bedingungen des Fremdsprachenunterrichts $\mathrm{zu}$ prüfen. Während die Kenntnisse abgefragt werden können, sind die Veränderungen der Einstellungen mit dieser Methode nicht zu prüfen. Es ist zwar möglich, die Einstellungen durch bestimmte Fragen abzuchecken, das wird aber nur eine subjektive Einschätzung des Lernenden selbst sein. Wie sich diese Einstellungen in der realen Situation des interkulturellen Kontakts offenbaren, ist schwer vorhersagbar.

Es ist wichtig zu wissen, wie sich die Lernziele im affektiven Bereich entwickeln können. 
Krathwohl 1964 (vgl. Mietzel 2007, S. 439-440) und seine Mitarbeiter haben folgende hierarchische Anordnung der Lernziele im affektiven Bereich herausgearbeitet:

- Aufnehmen

- Reagieren

- Werten

- Wertordnung

- Bestimmtwerden.

Wenn ein Sprachlernender im Rahmen des Fremdsprachenunterrichts mit interkulturellen Inhalten konfrontiert wird, dann wird ihm auf der ersten Ebene erstmals klar, dass solche Probleme existieren. Er wird gewahr, dass außerhalb der Sprache noch kulturelle Unterschiede als Störungen im Prozess der Kommunikation mit dem fremdkulturellen Partner auftreten können. Durch das geweckte Interesse wird aber der Lernende bereit sein, weitere Informationen aufzunehmen.

Auf der zweiten Ebene ist der Lernende bereit, nicht nur Informationen aufzunehmen, sondern auch auf sie zu reagieren. Er ist bereit, die Idee des interkulturellen Lernens zu übernehmen und zu vertreten. Er weiß, wie er in der interkulturellen Situation reagieren muss und was ihn zu einer erfolgreichen Kommunikation führen können. Er empfindet auch eine gewisse Befriedigung für seine Position.

Auf der dritten Ebene lässt sich schon die eigene Meinung erkennen. Der Lernende ist davon überzeugt, dass Toleranz, Respekt und Offenheit bei jedem interkulturellen Kontakt notwendig sind, beim Auftreten eines interkulturellen Missverständnisses sucht er nach möglichen Erklärungen und Lösungen des Problems in den interkulturellen Unterschieden oder in der eigenen (fremden) ethnozentrischen Position.

Auf Ebene vier wird der interkulturelle Gedanke als eigenständiger Wert anerkannt und in die eigene Wertordnung übernommen.

Auf der fünften Ebene „Bestimmtwerden“ ist es möglich, dass die interkulturellen Einstellungen in Verbindung zu anderen Werten gesetzt werden. Es wird z.B. anerkannt, dass die für das interkulturelle Lernen wichtigen Werte auch für jede menschliche Interaktion relevant sind. Da jeder Mensch eine eigene individuelle Kultur besitzt, kann jeder Akt der zwischenmenschlichen Kommunikation als interkultureller Kontakt beschrieben werden.

Die Entwicklung der affektiven Ziele der interkulturellen Kompetenz nach dem von Krathwohl vorgeschlagenen Modell wäre eines der möglichen Szenarien für die Herausbildung der affektiven Komponente. Die einzelnen Ebenen sind in diesem Modell nur theoretisch 
voneinander zu trennen, in der Praxis erscheint es jedoch problematisch zu bestimmen, auf welcher Ebene der Lernende sich bei der Entwicklung seiner Einstellungen befindet.

Ein anderes Modell der Entwicklung der affektiven Ziele im interkulturellen Bereich ist bei Bennett (1993) zu finden. Sein Modell beschäftigt sich unmittelbar mit den interkulturellen Inhalten und wird Developmental Model of intercultural Sensitivity genannt. Bennett definiert die Sensibilität ${ }^{27}$ anhand mehrerer Stadien des persönlichen Wachstums. Es handelt sich hier um ein Entwicklungsmodell, das die Veränderung der Einstellungen eines Menschen von ethnozentrischen bis zu ethnorelativistischen verfolgt.

„Interkulturelle Sensibilität zu entwickeln bedeutet demnach im Wesentlichen fundamentale Unterschiede in der Weltsicht der Kulturen zu erkennen und mit ihnen umgehen zu können“ (Martinelli 2000, S. 28)

In den ethnozentrischen Entwicklungsphasen geht der Mensch davon aus, dass seine eigene Weltsicht am meisten der Realität entspricht. Bennett unterscheidet zwischen drei ethnozentrischen Stadien:

- Leugnen. In diesem Stadium werden die kulturellen Unterschiede geleugnet. Es kann die Folge der Isolation oder der Trennung von den anderen kulturellen Gemeinschaften sein.

- Verteidigen. In diesem Stadium werden die kulturellen Unterschiede wahrgenommen und als Bedrohung der eigenen Einstellungen eingeschätzt. Infolgedessen werden sie bekämpft.

- Herunterspielen. Die kulturellen Unterschiede werden in diesem Stadium erkannt, ihre Bedeutung wird aber heruntergespielt. Die kulturellen Ähnlichkeiten, die meist aber auf grundlegende biologische Ähnlichkeiten zurückgehen, werden als viel gröBer und bedeutsamer empfunden, die Rolle der Unterschiede wird heruntergespielt. In den ethnorelativistischen Entwicklungsstadien geht der Mensch davon aus, dass bestimmte Verhaltens- und Denkweisen nur innerhalb einer Kultur verstanden werden können. Die Konfrontation mit einer anderen Kultur wird nicht als Bedrohung, sondern als Bereicherung verstanden. Es folgen die weiteren drei ethnorelativistieschen Entwicklungsstadien von Bennett:

- Akzeptieren. In diesem Stadium werden die kulturellen Unterschiede akzeptiert und respektiert. Im Weiteren müssen die eigenen Werte als kulturell geprägte anerkannt werden.

27 Es sei aber erwähnt, dass Bennett neben den emotionalen Elementen in seiner Konstruktbeschreibung auch kognitive und verhaltensbezogene Aspekte integriert. 
- Anpassen. Die Unterschiede zwischen der eigenen und der fremden Kultur werden hier als Möglichkeiten für die Bereicherung des eigenen kulturellen Potenzials betrachtet. Für dieses Stadium ist auch empathisches Herangehen charakteristisch.

- Integrieren. Die kulturellen Unterschiede werden in diesem Stadium positiv eingeschätzt, mehrere kulturelle Rahmen werden in einen einigen Rahmen integriert. unterschiedlichen Weltansichten werden in den eigenen Kontext hereingenommen und für die ständige Neudefinierung der eigenen Identität verwendet.

Das Entwicklungsmodell von Bennett ist klar strukturiert, was einen Maßstab für die Bestimmung der interkulturellen Entwicklung in einer Arbeitsgruppe vorgibt, aufgrund dessen die Trainingsmethoden ausgewählt werden können. Es liefert auch einen Überblick der Strategien, die in der interkulturellen Kontaktsituation üblich sind. Es ist aber fragwürdig anzunehmen, dass die Entwicklung eines Menschen alle Stadien in der Reihenfolge durchläuft, wie es im Modell vorgegeben ist. Es scheint auch unwahrscheinlich, dass ein Mensch, der sich in einem bestimmten Stadium befindet, nur die dazu gehörenden Strategien anwendet. Es ist plausibler anzunehmen, dass ein Mensch in unterschiedlichen Situationen unterschiedlich reagiert, d.h. sein Verhalten kann verschiedenen Stadien zugeordnet werden.

Die interkulturelle Sensibilität stellt die affektive Seite der interkulturellen Kompetenz dar. Bei der Entwicklung der interkulturellen Sensibilität wird mit den Emotionen gearbeitet, als Ziel ist die Veränderung der Einstellungen zu benennen. Die interkulturelle Sensibilität stellt einen Komplex aus unterschiedlichen Gefühlen, Einstellungen und Fähigkeiten dar, die den Menschen dazu befähigen, die Besonderheiten sowohl des Denkens, Fühlens und Handels des fremdkulturellen Partners als auch der kommunikativen Situation selbst wahrzunehmen und zu akzeptieren und davon abhängig sein Verhalten und seine Gefühle zu steuern.

Die interkulturelle Sensibilität schließt folgende Komponenten ein: Empathie; Bereitschaft, die eigenen kulturellen Normen zu relativieren; Offenheit und Respekt; Ambiguitätstoleranz.

Die Sensibilisierung oder die Entwicklung der interkulturellen Kompetenz ist ein langfristiger Vorgang, der auf Veränderungen der Einstellungen von einer ethnozentrischen zu einer ethnorelativistischen Position basiert. Im Fremdsprachenunterricht können quasi nur die Grundlagen der interkulturellen Einstellungen gelegt und die oben erwähnte Werte entwickelt werden. Die Weiterentwicklung der Sensibilität bedarf der interkulturellen Praxis. 
Dieser Prozess ist eng mit dem Prozess der Persönlichkeitsentwicklung verbunden, und deshalb ist es ein lebenslanger Lernprozess.

\subsection{Die interkulturellen Fähigkeiten als pragmatische Komponente}

Die dritte Komponente des Puzzle-Modells der interkulturellen Kompetenz vertritt die verhaltensbezogene Komponente. Sie besteht aus den interkulturellen Fähigkeiten. Kulturspezifische und kulturübergreifende Kenntnisse sind ebenso wichtig wie interkulturelle Sensibilität, sie reichen nicht automatisch für die Fähigkeit aus, adäquat und angemessen mit dem fremdkulturellen Partner kommunizieren zu können. Die verhaltensbezogene Komponente umfasst daher die operativen Fähigkeiten, um effektive interkulturelle Kommunikation gestalten zu können. Es muss erwähnt werden, dass die Separierung der einzelnen Elemente der interkulturellen Kompetenz rein theoretisch ist. Im Unterrichtsprozess entwickeln sich die einzelnen Komponenten häufig gleichzeitig und können nur theoretisch abgesondert werden.

Bloom nennt in seiner Taxonomie der Lernziele als dritte Komponente die Ziele im psychomotorischen Bereich, und obwohl diese Bezeichnung für den Fremdsprachenunterricht nicht ganz korrekt zu sein scheint, geht es dabei um Verhalten. Sowohl psychomotorische Fertigkeiten als auch sprachliche und interkulturelle Fähigkeiten finden ihren Ausdruck im Verhalten eines Menschen. Allein der Besitz von Kenntnissen und positiven Einstellungen macht den Menschen nicht fähig, sich angemessen zu verhalten. Ein Mensch, der die Grundregeln des Schwimmens in einem Schwimmkurs erlernt und auch alle emotionalen Barrieren überwunden hat, ist nicht unbedingt fähig zu schwimmen (vgl. Brislin und Yoshida 1994, S. 87). Das Gleiche gilt auch für interkulturelle Fähigkeiten und Fertigkeiten. Wenn jemand Fremdsprachenkenntnisse und Kenntnisse über die fremde Kultur hat, wenn ihm die Besonderheiten der interkulturellen Kommunikation bewusst sind und wenn er letztendlich positiv, offen und sensibel zu seinem fremdkulturellen Partner eingestellt ist, bedeutet dies noch nicht, dass dieser Mensch sich auch sensibel verhalten wird. Dazu sind Fähigkeiten notwendig, zum Beispiel die Fähigkeit, seine Kenntnisse in der konkreten Situation auch anzuwenden.

Fähigkeiten sind die „Gesamtheit der psychischen und physischen Bedingungen als Voraussetzung für die Ausführung von körperlichen oder geistlichen Leistungen“ (Böhm 2005, S. 2000). 
Fähigkeiten können angeboren und/oder durch Lern- oder Sozialisationsprozesse erworben werden. Häufig werden Fähigkeiten als Können einer bestimmten Handlung in einem bestimmten Gebiet definiert (vgl. Böhm 2005).

Furmanova (1993) vertritt die Meinung, dass in einem Unterrichtsprozess, dessen Ziel die Entwicklung der interkulturellen Kompetenz ist, neben dem kulturellen Grundwissen (Thesaurus I) auch der kulturelle Verhaltensmodus (Thesaurus II) zu entwickeln ist. Im Thesaurus II wird die Kultur des sprachlichen und außersprachlichen Verhaltens im Fremdsprachenunterricht geformt.

Die Entwicklung des kulturellen Verhaltensmodus bedeutet die Einübung der kommunikativen Fähigkeiten, die ihrerseits mit den interkulturellen Fertigkeiten verbunden sind, Informationen zu gewinnen und effektiv auf den Gesprächspartner einwirken zu können. Es wird zwischen Fähigkeiten und Fertigkeiten unterschieden. Während sich die Fähigkeit auf das Vermögen bezieht, eine Tätigkeit ausführen zu können, ist die Fertigkeit die automatisierte Komponente dieser Tätigkeit. Fertigkeit ist ein Können, etwas umzusetzen. Fertigkeiten sind durch Üben soweit mechanisiert bzw. automatisiert, dass sie ohne Einschalten des Bewusstseins vollzogen werden können. Der kulturelle Verhaltensmodus gewährleistet den Gesprächspartnern die Möglichkeit, einander richtig einschätzen und verstehen zu können (vgl. Furmanova 1993, S. 91).

Es geht bei Furmanova um die Entwicklung der Fähigkeiten, die die angemessene und effektive Kommunikation und Kooperation zwischen Vertretern unterschiedlicher Kulturen erlauben. Dabei betont sie die Fähigkeiten, die in der Fremdsprachendidaktik für die kommunikative Wende ${ }^{28}$ gesorgt haben. So erwähnt sie nach der Bestimmung der Intention des Gesprächspartners und dem Erzeugen der eigenen Sprachintention folgende Schritte: Bestimmung und Erfüllung der sozialen Rollen, die Feststellung der zwischenmenschlichen Beziehungen, Analyse der Situation. Alle oben erwähnten Schritte benötigen soziokulturelle Fähigkeiten, die sich auf das Wissen über die fremde und die eigene Kultur, aber auch über die Abhängigkeit der Kommunikation von äußeren Umständen erstrecken.

Der gemeinsame europäische Rahmen für Sprachen geht im Einzelnen auf Kenntnisse, Fähigkeiten und Fertigkeiten nicht ein. Hier wird im Allgemeinen das Wort „Kompetenzen“ bevorzugt. Unter diesem Begriff werden sowohl Kenntnisse als auch Fertigkeiten und Einstellungen zusammengefasst. Als oberstes Ziel des Fremdsprachenunterrichts wird im gemeinsamen Rahmen die kommunikative Kompetenz bezeichnet, alle anderen Kompeten-

28 Als kommunikative Wende wird ein in den 70er Jahren erfolgte Paradigmenwechsel im Fremdsprachenunterricht bezeichnet. 
zen werden hier als deren einzelne Aspekte betrachtet, die zwar viel zur Kommunikationsfähigkeit des Lernenden beitragen, aber trotzdem von den rein linguistischen Kompetenzen abgesondert werden.

Kompetenzen des Muttersprachlers oder des Sprachlernenden sind aus dieser Sicht:

Auszug aus dem Inhaltsverzeichnis des gemeinsamen europäischen Referenzrahmens für Sprachen (vgl. Trim 2006)

5. Die Kompetenzen des Sprachverwenders und des Sprachlernenden

5.1 Allgemeine Kompetenzen:

5.1.1.Deklaratives Wissen

5.1.2. Fertigkeiten oder prozedurales Wissen

5.1.3. Persönlichkeitsbezogene Kompetenz

5.1.4. Lernfähigkeit

5.2. Kommunikative Sprachkompetenzen:

5.2.1. linguistische Kompetenz

5.2.2. soziolinguistische Kompetenz

5.2.3. pragmatische Kompetenz

Interkulturelle Fertigkeiten begleitet von den praktischen Fertigkeiten (soziale, berufliche Fertigkeiten, Fertigkeiten für die Freizeit und für das tägliche Leben) fallen unter das Kapitel 5.1.2. „Fertigkeiten und prozedurales Wissen“.

$\mathrm{Zu}$ den interkulturellen Fähigkeiten gehören demnach:

- die Fähigkeit, die Ausgangskultur und die fremde Kultur miteinander in Beziehung zu setzen;

- die Fähigkeit, eine Reihe verschiedener Strategien für den Kontakt mit Angehörigen anderer Kulturen zu identifizieren und zu verwenden;

- die Fähigkeit, als kultureller Mittler zwischen der eigenen und der fremden Kultur zu agieren und wirksam mit interkulturellen Missverständnissen und Konfliktsituationen umzugehen;

- die Fähigkeit, stereotype Beziehungen zu überwinden.

Im gemeinsamen europäischen Rahmen für Sprachen wird der Versuch unternommen, die interkulturelle Seite des Sprachunterrichts mehr zu beachten, wobei kulturelle Unterschiede, Stereotype und interkulturelle Missverständnisse angesprochen werden. Es stellt sich jedoch die Frage, aufgrund welcher Kenntnisse diese Fähigkeiten gebildet werden sollen, 
wenn das Curriculum vorwiegend auf die sprachliche Seite ausgerichtet ist. Die Kenntnisse, die unter dem Begriff „Deklaratives Wissen“ (5.1.1.) aufgezählt sind, enthalten unter anderem zwar soziokulturelles Wissen und interkulturelles Bewusstsein, ${ }^{29}$ aber keine Kenntnisse über z.B. kulturelle Unterschiede oder Stereotype. Es bleibt ungeklärt, wie die interkulturellen Fähigkeiten, aber auch interkulturelles Bewusstsein entwickelt werden sollen.

Einen anderen Kritikpunkt stellt die Formulierung der Fähigkeiten selbst dar. Es werden Probleme, aber keine Lösungswege genannt. Z.B. „die Fähigkeit, stereotype Beziehungen zu überwinden“ ruft sofort die Frage „wie?“ auf. Was soll mit den Stereotypen geschehen, um sie überwinden zu können? Bei der Fähigkeit, eine Reihe verschiedener Strategien zu verwenden, ist die Frage ,welche Strategien?“ berechtigt.

Die Position der Herausgeber des Referenzrahmens bezüglich des interkulturellen Aspekts basiert auf folgender Überlegung: Rein interkulturelles Wissen gehört nicht unbedingt in den Fremdsprachenunterricht, es ist überflüssig, da es die Aufmerksamkeit der Sprachlernenden von der rein sprachlichen Seite ablenkt. Sie versuchen zwar dem Trend nachzugeben und die interkulturelle Seite einzubeziehen, weisen ihr aber einen Platz am Rande zu, ohne darauf einzugehen, wie die interkulturellen Fertigkeiten ohne entsprechendes Wissen im Unterricht entwickelt werden sollen. Als Gegenargument zu dieser Position kann daran erinnert werden, dass eine Fremdsprache dafür gelernt wird, um mit den Vertretern der anderen Kulturen möglichst reibungslos kommunizieren zu können. Also die Sprache wird gelernt, um in den interkulturellen Situationen agieren zu können, d.h. dass die interkulturellen Kenntnisse und Fertigkeiten unbedingt in solchen Situationen im Einsatz kommen. Das beweist m.E. die Notwendigkeit, interkulturelle Kenntnisse im Curriculum des Fremdsprachenunterrichts einzubeziehen. Dieses Thema wird deshalb gerade in Bezug auf den Referenzrahmen angesprochen, weil es eine Standardisierung im Bereich des Unterrichts der europäischen Sprachen erzielen, d.h. sie dienen als Orientierung für Praktiker, also Fremdsprachenlehrer.

Eine andere Sicht bezüglich der für die interkulturelle Kompetenz relevanten Fähigkeiten ist in den Arbeiten der interkulturellen Didaktik zu finden. Die unten aufgezählten Fähigkeiten beziehen sich weniger auf die Sprache als vielmehr auf die Prozesse, die in der Situation des interkulturellen Kontakts stattfinden. Sie beziehen sich auf die Art und Weise,

$29 \quad$ Interkulturelles Bewusstsein kann m.E. überhaupt nicht in eine Reihe mit den anderen Kenntnissen wie Weltwissen oder soziokulturelle Kenntnisse gestellt werden, weil dieser Begriff etwas bezeichnet, das mehr bedeutet und unter anderen Umständen entwickelt werden soll. 
wie das Wissen über die fremde und eigene Kultur verarbeitet werden kann. Zu den Kernfähigkeiten zählen dabei solche wie Zuhören, Beobachten, Analysieren, Bewerten, Zuordnen. Unter den 22 Elementen der interkulturellen Kompetenz, die am meisten die Zustimmung der Experten gefunden haben ${ }^{30}$, sind neun Elemente als Fähigkeiten zu identifizieren, und zwar:

- Fähigkeit sich selbst zu beurteilen,

- Anpassungsfähigkeit,

- Fähigkeit, zuzuhören und (aufmerksam) zu beobachten,

- Fähigkeit, sich wechselnden interkulturellen Kommunikations-- und Lernstilen anzupassen,

- Flexibilität,

- Fähigkeit, zu analysieren, zu deuten und zuzuordnen,

- Kognitive Flexibilität- Fähigkeit, das Referenzsystem etisch und emisch ${ }^{31}$ zu verlagern

- Ambiguitätstoleranz,

- Kulturübergreifende Empathie ${ }^{32}$.

Aus dieser Liste der Fähigkeiten wird deutlich, dass die interkulturellen Experten ihre Aufmerksamkeit auf die Fähigkeiten konzentrieren, die in der Situation eines idealen interkulturellen Kontakts vonnöten sind. Die Fähigkeit zur kommunikativen Kompetenz sowohl in einer Fremd- als auch in der Muttersprache wird außer Acht gelassen.

Es muss erwähnt werden, das die Rolle und die Bedeutung der Sprachbeherrschung für die interkulturellen Fertigkeiten bei dieser Studie ungeklärt blieb. Die Experten waren sich nicht einig, ob Sprachfähigkeiten und -fertigkeiten als notwendige Komponente der Kompetenz benannt werden sollen.

Werden die Meinungen der Didaktiker des Fremdsprachenunterrichts und der Experten im

30 Die Untersuchung wurde im Auftrag der Bertelsmann Stiftung von Dr. Carla K. Deardorff durchgeführt. Dabei wurden interkulturelle Experten und Hochschullehrenden befragt, welche Elemente der interkulturellen Kompetenz besonders wichtig sind. Es wurden 22 Teilelemente genannt, denen 80-100\% der Experten zugestimmt hatten.

31 Die Begriffe ,etisch“ und „emisch“ stammen ursprünglich aus der Linguistik, werden aber zur Zeit meist in der Ethnologie verwendet. Emische Erforschung einer Kultur bedeutet, dass diese Kultur mit den Augen eines Insiders aus der Innenperspektive beurteilt wird, im Gegensatz $\mathrm{zu}$ der etischen Herangehensweise, wobei eine Kultur von einem fremden Beobachter anhand der mitgebrachten Kriterien beurteilt wird.

32 Die zwei letzten Elemente Ambiguitätstoleranz und Empathie gehören m.E. zum Bereich „Emotionen“, sind aber formal als Fähigkeiten definiert und deshalb in der Liste der am häufigsten erwähnten Fähigkeiten eingetragen. 
interkulturellen Bereich zusammengefasst, lassen sich folgende Fähigkeiten unterscheiden, die für einen Fremdsprachenlernenden notwendig sind und deshalb im Fremdsprachenunterricht entwickelt werden müssen:

Kommunikationsfähigkeit (Kompetenz). Diese Fähigkeit ist hier sowohl bezüglich einer Fremdsprache als auch bezüglich der Muttersprache gemeint. Die Kommunikationsfähigkeit wird in der Literatur meist als Kompetenz in der Muttersprache gemeint.

„Kommunikative Kompetenz ist die menschliche Fähigkeit zur Sprachverwendung, die nicht nur die grammatischen Kenntnisse, sondern auch die Regeln der Sprachverwendung in einem gegebenen soziokulturellen Raum beinhaltet" (Brockhaus 1996, Band 12, S. 228). Kommunikationsfähigkeit ist eine Fähigkeit, einerseits sich klar und verständlich auszudrücken und sein Anliegen deutlich und transparent zu übermitteln, andererseits den Gesprächspartnern aktiv und aufmerksam zuhören zu können und das, was sie sagen, zu verstehen und einzuordnen.

Diese zwei Pole der Kommunikationsfähigkeit lassen sich aus dem zweiseitigen Charakter der Kommunikation ableiten, wobei ein und derselbe Teilnehmer gleichzeitig Sender und Empfänger der Informationen oder der Botschaften ist. Diese Botschaften werden nicht nur in der Form verbaler Botschaften gesendet, Medien können dabei mehrere Faktoren sein: Mimik, Gestik, Körperhaltung, Lautstärke etc. Kommunikativ zu sein bedeutet, als Sender zu verstehen, welche Botschaften diese Medien an den Empfänger weiterleiten. Der kommunikationskompetente Empfänger muss imstande sein, die oben erwähnten Botschaften $\mathrm{zu}$ entschlüsseln und entsprechend darauf zu reagieren.

Die Kommunikationsfähigkeit in einer Fremdsprache bzw. in einer fremden Kultur verläuft unter anderen Bedingungen, und ist dadurch erheblich erschwert. Der fachliche Ausdruck, der in der Fremdsprachendidaktik für die Kommunikationsfähigkeit in einer fremden Sprache verwendet wird, ist die kommunikative Kompetenz. Seit den 70er Jahren wird sie als Ziel des Fremdsprachenunterrichts benannt. ${ }^{33}$ Als wichtigste Komponente der kommunikativen Kompetenz ist natürlich die Sprachkompetenz zu benennen. Die kommunikative Sprachkompetenz hat aber viel mehr Facetten als nur die Beherrschung der linguistischen Seite der Sprache. Die Kompetenzen in den soziolinguistischen und pragmatischen Bereichen vervollständigen das Gesamtbild.

33 Die Zusammenhänge und Unterschiede zwischen der kommunikativen und interkulturellen Kompetenz und ihre Berechtigung, als Ziel des Unterrichts genannt zu werden, wurde im Kapitel 2.2 beschrieben. 
Tabelle 2: Kommunikative Sprachkompetenzen (Quelle: Trim 2006, Gemeinsamer europäischer Referenzrahmen für Sprachen)

\begin{tabular}{|c|c|c|}
\hline \multicolumn{3}{|c|}{ Kommunikative Sprachkompetenzen } \\
\hline Linguistische Kompetenzen & $\begin{array}{c}\text { Soziolinguistische Kompe- } \\
\text { tenzen }\end{array}$ & Pragmatische Kompetenzen \\
\hline $\begin{array}{l}\text { - lexikalische } \\
\text { - grammatische } \\
\text { - semantische } \\
\text { - phonologische }\end{array}$ & $\begin{array}{l}\text { - sprachliche Kenn- } \\
\text { zeichnung sozialer } \\
\text { Beziehungen } \\
\text { - Höflichkeitskon- } \\
\text { ven-tionen } \\
\text { - Redewendungen, } \\
\text { Aussprüche, Zitate } \\
\text { und sprichwörtliche } \\
\text { Redensarten } \\
\text { - Registerunterschiede } \\
\text { - Varietäten }\end{array}$ & $\begin{array}{l}\text { - Diskurskompetenz } \\
\text { - funktionale Kompe- } \\
\text { tenz } \\
\text { - Schemakompetenz }\end{array}$ \\
\hline
\end{tabular}

An dieser Stelle ist folgende Frage berechtigt: Wenn die Kommunikation mit einem fremdkulturellen Partner in einer Fremdsprache geführt wird, ist dann wirklich die Kommunikationsfähigkeit in der Muttersprache notwendigerweise besser? Die Erfahrung zeigt, dass Menschen, die in ihrer Muttersprache Probleme bei der Kommunikation mit anderen haben, höchstwahrscheinlich auch Probleme haben, ihre Gedanken und Intentionen in der Fremdsprache klar und deutlich auszudrücken. Hier ein Beispiel: Als Lehrkraft in einem Alphabetisierungskurs hatte die Verfasserin Probleme, eine Kursteilnehmerin aus der Türkei zu verstehen. Es lag daran, dass diese oft nicht aufpasste, welche Frage gestellt wurde. Außerdem hatte die Teilnehmerin Schwierigkeiten, ihre Gedanken auszudrücken. Im Gespräch mit einer Kollegin türkischer Herkunft stellte sich jedoch heraus, dass die oben erwähnte Teilnehmerin auch in der Muttersprache nur einfache Sätze verstehen konnte und auch nur mit einfachen Sätzen antwortete. ${ }^{34}$ Wer also ein Gespräch in der Muttersprache erfolgreich führen kann, kann auch eher in einer interkulturellen Situation kompetent kommunikativ handeln.

34 Die Behauptung, dass allein die schlecht entwickelte Kommunikationskompetenz in der türkischen Sprache der Grund für das mühsame Vorankommen bei kommunikativen Erfolgen in der deutschen Sprache war, ist nicht konsequent. Dafür gab es sicherlich auch andere Gründe. Aber eine gewisse Abhängigkeit vom sprachlichen Niveau in der Muttersprache ist nicht zu leugnen. 
Eine weitere Frage wäre, ob die gut entwickelte Kommunikationsfähigkeit in der Muttersprache der Grund für falsche Interpretationen bzw. Reaktionen sein kann. Wie schon beschrieben wurde, werden kommunikative Botschaften nicht nur in der Form sprachlicher Äußerungen, sondern auch durch Mimik, Gestik etc. gesendet. Und das birgt ein gewisses Potenzial an Missverständnissen, da gerade auf diesem Weg empfangene Botschaften mit unseren in der Mutterkultur erworbenen Kriterien interpretiert werden. Hierbei leisten Hilfe weitere interkulturelle Fähigkeiten, wie die Fähigkeit zur Selbstwahrnehmung und die Fähigkeit zum interkulturellen Vergleich, die unten ausführlicher beschrieben werden. An dieser Stelle ist anzumerken, dass sich die erforderliche Kommunikationsfähigkeit nicht nur auf eine zu erlernende Fremdsprache bezieht, sondern auch auf die Muttersprache.

Die Bedeutung der Muttersprache bzw. der Mutterkultur darf nicht unterschätzt werden. Die Zusammenhänge, Einstellungen und Deutungsmuster, die während des Sozialisationsprozesses erlernt wurden, dienen als Grundlage für Interpretationen des Geschehenen. Man betrachtet sie als Selbstverständlichkeiten, auf die man sich verlassen kann. Bei der Konfrontation mit einer fremden Kultur bzw. mit dem Vertreter einer fremden Kultur sind gerade diese Deutungsmuster hinderlich. Sie bilden häufig eine falsche Interpretationsplattform, mit der man bei dem Verständigungsprozess einfach nicht weiterkommt. Falsche Interpretationen haben falsche Reaktionen zur Folge, was weiter zu Missverständnissen führt. Aus diesem Grund gehört zur interkulturellen Kompetenz eine weitere Fähigkeit, die

\section{Fähigkeit zur Selbstreflexion}

Reflexion (von lat. reflexio - „das Zurückbeugen, -biegen, -krümmen) ist eine Form der menschlichen Tätigkeit, die auf das Bewusstmachen des eigenen Verhaltens und der eigenen Einstellungen gerichtet ist (vgl. Ridkina 2000, S. 108). Im Sinne einer interkulturellen Situation richtet sich die Reflexion auf die kulturellen Hintergründe ihrer Teilnehmer, die für Deutungen, Reaktionen und Verhalten verantwortlich sind.

„Kulturelle Selbstreflexion ist das Nachdenken darüber und das Bewusstsein davon, was ich selbst in ,kultureller Sicht' bin. Es geht also um das Erkennen der Einflüsse und Prägungen, die ich durch Kulturgemeinschaften erfahren habe, denen ich angehörte und noch angehöre. Es geht darum, dass ich mir der kulturellen Orientierungen und der kulturellen Schemata bewusst werde, die meine Wahrnehmung und mein Handeln bestimmen. Und es geht darum, dass ich mir Klarheit verschaffe über den kulturellen Hintergrund, in den ich hineingewachsen bin, den ich als ,selbstverständlich“ und ,normal ‘ empfinde. Und schließlich geht es darum, dass ich den kulturellen Bezugsrahmen kennen lerne, der es mir ermög- 
licht, ,Eigenes“ von ,Fremdem‘ zu unterscheiden“ (Flechsig 2001, S. 107).

Wie relevant ist die Fähigkeit zur Selbstreflexion für den Fremdsprachenunterricht? Die Bedingungen solchen Unterrichts im Ausland sind so, dass die Gruppen der Teilnehmer hinsichtlich des kulturellen Ursprungs relativ homogen sind (im Unterschied zum Zweitsprachenunterricht im Inland, wo die Gruppen heterogen sind und jeder Teilnehmer quasi gezwungen ist, die eigene kulturelle Identität jeden Tag mit den Studienkollegen im Kurs und außerhalb des Unterrichts zu erleben und wahrzunehmen). Da die Lernenden in den (relativ) homogenen Gruppen sich auch als einheitliche Gruppe verstehen, nehmen sie etwas Fremdes, in diesen Fall fremde Kultur bzw. fremde kulturelle Sachverhalte, als etwas Merkwürdiges, Komisches, Defizitäres wahr. Die Unfähigkeit, das Eigene zu erkennen, führt zu unreflektierter Herabsetzung des Fremden. Es ist die Aufgabe des Lehrers zu zeigen, dass hinter dem eigenen Verhalten auch kulturgefärbte Interpretationen stehen. Es gibt keine neutrale Wahrnehmung, keine neutralen Deutungsmuster. Wenn das Fremde ein Unterrichtsinhalt ist, soll das Eigene dies ebenso werden.

Ein weiterer Schritt dabei wäre, den Lernenden, die sich als homogene Gruppe verstehen, zu zeigen, dass Homogenität innerhalb einer (nationalen) Kultur nur scheinbar ist: Innerhalb einer (nationalen) Kultur gibt es immer weitere kulturelle Unterschiede, die durch die Zugehörigkeit der Lernenden zu unterschiedlichen Bezugsgruppen oder Kulturkreisen zu erklären sind und die ihr Verhalten unterschiedlich beeinflussen. Neben der Akzentuierung der Unterschiede innerhalb seiner eigenen Kultur muss aber auch die Suche nach den Gemeinsamkeiten mit der fremden Kultur stattfinden, damit der Lernende sich nicht nur mit der eigenen Kultur identifiziert, sondern sich auch als Weltbürger wahrnimmt. Dies wären aber schon weitere Schritte der Persönlichkeitsentwicklung.

Selbstreflexion bezüglich der Unterrichtsinhalte geschieht auf keinen Fall automatisch. Es genügt nicht der einfache Vergleich der Sachverhalte in der eigenen Kultur und in der Kultur der erlernten Sprache. Ein einfacher Vergleich des Fremden mit dem Vertrauten führt leicht zu Besser-Schlechter-Bewertungen (vgl. Richter 1998). Zur Schulung der Selbstwahrnehmung trägt die Analyse der Sachverhalte einer fremden Wirklichkeit, bei der den Lernenden die Möglichkeit gegeben wird, auf eigene kulturelle Quellen zurückzugreifen und diese dann in Anbetracht des Fremden in Frage zu stellen, wesentlich mehr bei. Der Sachverhalt wird aus mehreren Perspektiven betrachtet, analysiert, bewertet. Und dafür wird eine weitere Fähigkeit benötigt und zwar die 


\section{Fähigkeit zur konstruktiven Auseinandersetzung mit dem Eigenen und dem Fremden}

Wenn bei der oben beschriebenen Fähigkeit von der Kategorie „das Eigene“ die Rede war, hat die dritte Fähigkeit zwei Pole: „das Fremde“ und „das Eigene“. Es geht hier darum, in unterschiedlichen Sachverhalten die beiden Pole wahrzunehmen, zu reflektieren, miteinander in Verbindung zu bringen und zu analysieren, um entsprechende Verhaltensstrategien auswählen zu können. „Konstruktiv“ hat in diesem Zusammenhang die Bedeutung von „produktiv“, „,örderlich“.

Schon die Aufzählung der erwünschten Handlungen zeigt, dass diese Fähigkeit ein Sammelbegriff ist und eine Reihe von Teilkompetenzen erfordert.

Eine der wichtigsten ist die Fähigkeit zur Perspektivenübernahme. Dazu ein Beispiel: „Nein“ wehrte der Mann erschrocken ab, als er in der Türkei gefragt wurde, ob er Ausländer sei. „Ich bin ein Deutscher“ (vgl. Szablewski-Cavus o. J., S. 4).

In den Situationen, in denen Missverständnisse mit anderen Leuten entstehen, fehlt häufig der Blick von der anderen Seite. Dies gilt auch innerhalb einer Kultur, im besonderen Maße ist es aber notwendig bei der Konfrontation von einer oder mehreren Kulturen. Es liegt daran, dass die Betrachtungs- und Interpretationsweise der Kommunikationspartner häufig sehr weit auseinanderliegen. Die Fähigkeit, den eigenen kulturellen Standpunkt gegen den des fremdkulturellen Kommunikationspartners zu wechseln, wird „Fähigkeit zur Perspektivenübernahme“" genannt.

Der Unterricht in monokulturellen Gruppen lässt wesentlich weniger Spielraum für solche Übungen als der Unterricht in internationalen Gruppen, wo die unterschiedlichen Perspektiven ins Auge fallen. Trotzdem bietet eine gezielte Auswahl von Texten, Übungen, Diskussionsthemen und -fragen Möglichkeiten, die fremde Perspektive auszuprobieren. Man lernt dabei, von den eigenen „selbstverständlichen“ Standpunkten abzuweichen, keine vorschnellen Bewertungen oder Beurteilungen zu treffen, bei der Entstehung von interkulturellen Missverständnissen gezielt nach dem Hintergrund der Verhaltensweisen des fremdkulturellen Partners zu fragen.

Eng damit verbunden ist die Fähigkeit zur Rollendistanz. Um den Blick aus der fremden Perspektive zu gewinnen, muss die Person fähig sein, sich von der eigenen Rolle zu distanzieren. Dank dieser Fähigkeit kann sie erkennen, dass der Einzelne in einer interkulturellen Situation nicht nur die Rolle als Träger einer Kultur spielt, sondern auch viele weitere Rollen, wie z.B. die Rolle eines Studenten, eines Familienvaters, einer Frau etc. Mit jeder dieser Rollen sind unterschiedliche, oft auch widersprüchliche Sichtweisen verbunden. Dabei sind auch die Erwartungen an den Menschen als Träger unterschiedlicher Rollen nicht ein- 
deutig. Es gibt unterschiedliche Erwartungen, was die Denkweise oder das Verhalten anbetrifft, an einen Studenten oder an einen Familienvater. Solche Einschätzungen basieren auf vorhandenen Vorurteilen. Diese zu erkennen, sich von der kulturellen als der alleinigen Rolle $\mathrm{zu}$ distanzieren (,,ich trete in einer interkulturellen Kommunikationssituation nicht nur als eine Deutsche, eine Russin auf, sondern auch als Studentin, Mutter und Frau“), die unterschiedlichen Rollen beim Gesprächspartner zu erkennen - das bedeutet die Fähigkeit zur Rollendistanz.

„Unter ,Rollendistanz“ versteht man die Fähigkeit, sich gleichsam selbst ,auf den Kopf gucken', sich also in seinem eigenen Handeln beobachten zu können. Damit vergegenständlicht man in gewisser Weise natürlich auch den gesamten (interkulturellen) Handlungskontext, was es erleichtert, die Differenz zwischen Eigenem und Fremdem zu reflektieren. Selbstbeobachtung in diesem Sinne ist letztlich auch eine Grundlage für fremdkontrolliertes Handeln, was keineswegs auf Emotionslosigkeit hinauslaufen soll oder muss“ (Bolton 2007, S. 143).

Die Fähigkeiten zur Perspektivenübernahme und zur Rollendistanz fördern die reflektierende Vergleichskompetenz. Im Unterschied zum Alltagsvergleich geht ein reflektiertes Vergleichen über die Bewertungen und Beurteilungen hinaus. Bei reflektierendem Vergleich sucht man sowohl nach den Unterschieden als auch nach den Gemeinsamkeiten. Dabei werden keine vorschnellen Entscheidungen getroffen, was das Verhalten des Kommunikationspartners anbetrifft, sondern es wird auch der Versuch unternommen, das Hintergrundwissen über dieses Verhalten mit dem eigenen „selbstverständlichen“ Wissen zu vergleichen. Die Entwicklung der Fähigkeit zum reflektierten Vergleich ist besonders wichtig, aber auch besonders schwierig in einem Fremdsprachenunterricht im Ausland. Da fehlt oft ein direkter Kontakt zur Sprachkultur, deshalb sind die Bilder der fremden Kultur, der fremden Realität, die durch Medien wie Bücher, Videos oder Meinungen anderer Menschen vermittelt worden sind, durch Stereotype und Vorurteile verzerrt. Die aber haben eindeutig eine bewertende (manchmal auch abwertende) Funktion. Das Hinterfragen des Ursprungs, der Grundposition erlaubt diese bewertende Wirkung zu schwächen oder verschwinden zu lassen, was den Vergleich der kulturellen Verhaltensweisen sachlicher macht und dann positiv weiter auf das gesamte Kommunikationsbild wirkt.

Als letzte in diesem Bündel der nah beieinanderliegenden Teilkompetenzen, die für eine konstruktive Auseinandersetzung mit dem Fremden und dem Eigenen wichtig sind, ist die Fähigkeit zur Vermittlung zwischen der fremden und der eigenen Kultur zu nennen. Nach Schinschke (1995) setzt sie ,ein Bewusstsein von möglichen Gemeinsamkeiten, Ähnlich- 
keiten und Unterschieden voraus, nicht die Informiertheit über alle tatsächlichen Differenzen“ (Schinschke 1995, S. 38). Diese Fähigkeit bezieht sich vor allem auf die Kommunikation zwischen fremdkulturellen Gesprächspartnern. Um wie ein Vermittler zwischen der eigenen und der fremden Kultur agieren zu können, muss der zweiseitige Charakter solcher Kommunikation beachtet werden. Vermitteln in diesem Sinne heißt Aushandeln, und zwar zwischen den unterschiedlichen Denk- und Verhaltensweisen, zwischen den unterschiedlichen Kommunikationsstilen. Nicht nur das Fremde wird als Eigenständiges erkannt, akzeptiert und grundsätzlich respektiert, und nicht nur das mit dem eigenen kulturellen Hintergrund Identische bleibt. Beim Aushandeln wird ein neuer Raum geschaffen, wo kommunikations- und kulturbedingte Probleme thematisiert werden und nach gemeinsamen Wegen zum Umgang mit ihnen gesucht wird.

Trotz gut entwickelter Kommunikationsfähigkeit und trotz der Fähigkeiten zur Selbstreflexion und zum konstruktiven Umgang mit dem Fremden und dem Eigenen kann es in einer interkulturellen Kommunikationssituation dazu kommen, dass die Gesprächspartner einander nicht verstehen. Die Gründe dafür liegen nicht nur im mangelhaften Beherrschen der sprachlichen Äußerungen im engeren Sinne. Der gemeinsame Kontext, sei er beruflicher, familiärer oder kultureller Natur, erleichtert die Kommunikation. Das Nichtvorhandensein des gemeinsamen (kulturellen) Kontextes führt zu Missverständnissen. Sie dienen manchmal als Barometer für den Erfolg der interkulturellen Kommunikation. Deshalb ist auf der Handlungsebene der interkulturellen Kompetenz eine weitere Fähigkeit erforderlich, die

\section{Fähigkeit zur Vermeidung und Klärung von Missverständnissen}

Diese Fähigkeit basiert in erster Linie auf der Kenntnis der potenziellen Quellen für Missverständnisse. Die Beherrschung der kulturspezifischen Kenntnisse, die schon im Kapitel 1.4.1.1 beschrieben wurden, und die Fähigkeit, diese Kenntnisse mit denen vergleichen zu können, die zu den entsprechenden Themen in der Mutterkultur existieren, machen einen Teil dieser Fähigkeiten aus. Es hilft den Gesprächspartnern, mit Themen sensibel umzugehen, die zum Beispiel im Bereich von Tabus liegen. Eine andere Möglichkeit, Missverständnisse zu vermeiden ist ein bewusster Umgang mit den Kommunikationsstilen. Die Gesprächsstile der einzelnen Personen werden in Abhängigkeit von dem Kulturkreis entwickelt und haben deshalb einen gewissen Einfluss auf den Erfolg der Kommunikation. „In verschiedenen Kulturkreisen werden einzelne ,Parameter ${ }^{6}$ von Kommunikationen unterschiedlich gewichtet" (Szablewski-Cavus, S. 7). Die Unterschiede im kommunikativen Verhalten des Gesprächspartners zu erkennen und auf seine Kommunikation einzugehen, 
ist eine der Möglichkeiten, interkulturelle Missverständnisse zu vermeiden.

Wenn aber Missverständnisse in einer interkulturellen Situation schon entstanden sind, muss man fähig sein, sie als solche zu identifizieren und zu analysieren. Nicht selten wird ein Missverständnis von einem der Gesprächspartner gar nicht erkannt. Eine noch schlimmere Möglichkeit ist, wenn eine misslungene Kommunikation den bösen Absichten des fremdkulturellen Partners zugeschrieben wird. Um solche Missinterpretationen zu vermeiden, müssen die existierenden Unterschiede in den heimischen Kulturen bzw. ihre Auswirkungen auf kommunikatives Handeln und Verhalten anerkannt, analysiert und bearbeitet werden. Ein weiterer Schritt in der Arbeit mit Missverständnissen wäre die Erarbeitung und Aneignung von Strategien zur Vermeidung und Klärung von Missverständnissen in der Kommunikation (vgl. Knapp und Knapp-Potthof 1990, S. 84-85).

Zusammenfassend lässt sich Folgendes sagen: Die verhaltensbezogene Komponente der interkulturellen Kompetenz schließt Fähigkeiten ein, die die Lernenden erlauben, auf der Grundlage der Kenntnisse und mit Hilfe der interkulturellen Sensibilität adäquat und erfolgreich in den Situationen interkultureller Kontakte zu kommunizieren Zu den Fähigkeiten, die im interkulturellen Kontext notwendig sind, gehören:

- Kommunikationsfähigkeit

- Fähigkeit zur Selbstreflexion

- Fähigkeit zur konstruktiven Auseinandersetzung mit dem Eigenen und dem Fremden

- Fähigkeit zur Vermeidung und Klärung von Missverständnissen

- Fähigkeit zum Umgang mit vorgefassten Meinungen (Stereotypenkompetenz).

\section{Programm zur Entwicklung der interkulturellen Kompetenz}

\section{1 Einführung in das Programm: Ziel und Aufgaben}

Im Kapitel 3 wurden die wichtigsten Bestandteile der interkulturellen Kompetenz diskutiert: Die kulturspezifischen und kulturübergreifenden Kenntnisse stellen die kognitive Komponente dar, die interkulturelle Sensibilität ist die Verwirklichung der affektiven Seite und die interkulturellen Fähigkeiten und Fertigkeiten sind für die Realisierung des pragmatischen Aspekts verantwortlich. Das von der Autorin erarbeitete und diskutierte PuzzleModell bildet den theoretischen Hintergrund für das Programm zur Entwicklung der inter- 
kulturellen Kompetenz. Es folgt nun der nächste Schritt: die Umsetzung dieses Konzepts in der Praxis.

Im vorigen Kapitel wurde bereits die Tatsache angesprochen, dass das Konzept der interkulturellen Kompetenz in drei Bereichen eingesetzt wird: als eine Herausforderung der interkulturellen Gesellschaft, als eine der wichtigsten Charakteristika von Mitarbeitern in internationalen Unternehmen und als neues Ziel des Fremdsprachenunterrichts. Die gut ausgearbeitete und erprobte praktische Umsetzung des Konzepts ist aber nur im Bereich des interkulturellen Managements zu finden (vgl. Thomas 1993, Yoosefi und Thomas 2008; Bolten 2007, Hall und Hall 1989, Brislin und Yoshida 1994). Die meisten Schulungsangebote, die zur Zeit auf dem freien Bildungsmarkt existieren, richten sich an Interessenten, die in einem internationalen Unternehmen tätig sind oder durch ihre berufliche Tätigkeit mit Vertretern anderer Nationen konfrontiert werden.

In den anderen zwei Bereichen sind einzelne Ausarbeitungen oder Projekte bekannt. So wird der interkulturelle Gedanke in einer multinationalen Gesellschaft durch unterschiedliche Initiativen oder Projekte vermittelt. Die meisten davon sind jedoch entweder für Mitbürger ausländischer Herkunft oder für deren Kinder bestimmt, sie agieren unter dem Motto: „Lernen durch gemeinsames Handeln“ und sind dennoch vorwiegend auf Wissensvermittlung ausgerichtet.

Was den Fremdsprachenunterricht anbetrifft, sieht die Situation folgendermaßen aus: Das Prinzip des interkulturellen Lernens im Rahmen des Sprachlernens hat sich in der Theorie durchgesetzt. In der Praxis wird versucht, dieses Prinzip durch neue Inhalte und neue Akzente umzusetzen. So haben landeskundliche Informationen eine neue Bedeutung als Kulturvermittler gewonnen. Der Schwerpunkt wird jetzt von allgemeinen Informationen über Geografie, Geschichte etc. auf das Alltagswissen und die fremdkulturellen Orientierungssysteme wie Normen oder Einstellungen übertragen. Es werden auch neue, für das interkulturelle Lernen relevante Themen im Curriculum eingeführt, besonders deutlich ist dies beim Thema „Stereotype“ zu sehen, z.B. in den Lehrbücher „Themen neu“ (Aufderstraße, Bock, Müller und Müller 2000), „Schritte 6“(Hilpert et al., 2009).

Es gibt auch weitere Materialien im Bereich „Deutsch als Fremdsprache“, die sich mit der Umsetzung des interkulturellen Gedankens im Deutschunterricht beschäftigen, z.B. unter Themen wie: Sprachbrücke, Sichtwechsel, zwischen den Kulturen, typisch deutsch?

Eine weitere Anpassungsstrategie, die für das Ziel „Interkulturelle Kompetenz“ in der Praxis des modernen Fremdsprachenunterrichts immer mehr an Bedeutung gewinnt, ist das Vergleichen als Arbeitsmethode. Kenntnisse aus dem Alltag der fremden Kultur werden 
nicht nur mitgeteilt, sondern auch mit dem Wissen aus dem eigenen kulturellen Alltag verglichen, wie z.B. Verhaltensregeln bei einem Besuch oder beim Schenken/Bekommen eines Blumenstraußes. Dies hat dazu geführt, dass die eigene Kultur des Lernenden eine neue Position im Unterricht gefunden hat. ${ }^{35}$

Auch die Gestaltung der Lehrbücher hat sich dem interkulturellen Kontext angepasst. Kenntnisse der fremden nationalen Kultur werden viel differenzierter vermittelt , d.h. es werden verschiedene regionale Kulturen und auch deren Subkulturen in den Blick genommen, wie z.B. die Subkultur von Jugendlichen. Im Unterschied zu den alten Lernbüchern achten die Verfasser immer mehr darauf, die vorhandenen Stereotype abzubauen und dabei keine neuen Vorurteile zu vermitteln, so z.B. agieren in den Texten deutsche Bürger mit Migrationshintergrund oder es werden verschiedene Arten des Alltagslebens gezeigt (Leben in der Stadt oder auf dem Land).

Zusammenfassend kann man sagen, dass bereits einige praktische Erarbeitungen zum interkulturellen Kontext im Rahmen des Fremdsprachenunterrichts vorhanden sind. Sie gründen sich auf die für die Fremdsprachendidaktik typischen Methoden und Inhalte. Die Orientierung auf die Sprache und die Landeskunde liegt diesen Erarbeitungen zugrunde.

Das reiche Potenzial an Lehransätzen und Lehrmaterialien, die in der interkulturellen Didaktik und im interkulturellen Training gängig sind, wird im Fremdsprachenunterricht meistens außer Acht gelassen.

Diese Tatsache lässt sich dadurch erklären, dass die beiden Richtungen zwar interkulturelle Kompetenz als Ziel anerkennen, aber unterschiedliche wissenschaftliche Hintergründe haben: Während die interkulturelle Didaktik ihren Ursprung in der Psychologie hat, basieren die Arbeiten der Fremdsprachendidaktik auf Lehren aus der Sprachforschung, Linguistik, Literaturwissenschaft und Pädagogik. Statt das gesamte Wissen der unterschiedlichen Gebiete $\mathrm{zu}$ vereinen und sich gegenseitig nicht nur durch Ideenwechsel, sondern auch Austausch von Arbeitsmethoden und Arbeitsmaterialien $\mathrm{zu}$ befruchten, weisen die beiden Fachdisziplinen auf gegenseitige Nachteile und Mängel hin.

„The goal then, of intercultural competence, concerns both language and intercultural areas. With rare exception, interculturalists often overlook (or leave it to language teachers) the task of developing language competence, just as teachers overlook (or leave it to interculturalists) the task of developing intercultural abilities despite wide acknowledgement

35 Dies geschieht im Gegensatz zu den Konzepten der 80er Jahre, als Fremdsprachenlehrer dazu aufgefordert waren, im Unterricht ausschließlich die Fremdsprache zu benutzen und sich keine Anmerkungen in der Muttersprache zu erlauben. Das führte automatisch zum Verdrängen der Muttersprache aus dem Unterrichtsraum. Das Konzept aber war zum Scheitern verurteilt, weil es unmöglich war, die eigene Kultur aus den Köpfen der Lernenden zu unterdrücken. 
that language and culture are dimensions of each other, interrelated and inseparable" (Fantini 1995: 143, zitiert nach Bosse 2003, S. 158)

Da die beiden Fachbereiche sich schon auf der theoretischen Ebene als Rivalen sehen und auf die Kooperation miteinander nicht eingehen wollen, nutzen sie auch ihre Potenziale für die praktische Arbeit nicht aus. Die breite Palette von praktischen Materialien, die aus den interkulturellen Trainingsmaßnahmen bekannt sind, wird kaum für den Fremdsprachenunterricht eingesetzt. Die Ablehnung der Methoden des interkulturellen Trainings führt dazu, dass einige wichtige Aspekte der interkulturellen Kompetenz vernachlässigt werden. Während bei den sprachlichen Informationen und der Landeskunde die Wissensvermittlung ausreichend sein kann, benötigen die interkulturellen Fähigkeiten und insbesondere die interkulturelle Sensibilität andere Vorgehensweisen. Bei Letzterer sind z.B. die Analyse kritischer Ereignisse und die Selbstreflexion als erprobte Methoden aus der interkulturellen Didaktik vonnöten.

Das in dieser Dissertation vorgeschlagene Programm stellt den Versuch dar, die Methoden und die Inhalte der interkulturellen Didaktik in den Fremdsprachenunterricht zu integrieren. Der allgemeine Fremdsprachenunterricht, der bereits landeskundliche Informationen aus der Kultur der erlernten Sprache in sich schließt, wird durch zusätzliche Materialien aus den interkulturellen Trainings ergänzt.

Das Ziel des von der Verfasserin erarbeiteten Programms zur Entwicklung der interkulturellen Kompetenz besteht darin, durch und mit Hilfe einer Fremdsprache und mit interkulturell bedeutsamen Informationen und Handlungen die Lernenden auf Situationen des interkulturellen Kontakts vorzubereiten. Dabei müssen sie die eigenen kulturellen Einstellungen reflektieren, die kulturellen Unterschiede und Gemeinsamkeiten wahrnehmen und sensibel darauf reagieren, indem sie situations-, ziel- und partnergerecht kommunizieren und handeln.

Die enge Verbindung und Wechselwirkung zwischen Sprache und Kultur ist bei der Erarbeitung des Programms zu berücksichtigen. Die Entwicklung der kommunikativen Fähigkeiten, die durch Arbeit am Wortschatz, der Phonetik, den grammatikalischen Regeln erfolgt, wird durch landeskundliche Informationen, wie z.B. situationsbezogene Verhaltensweisen, Kenntnisse über die Begrüßungs- oder Verabschiedungsformeln etc. ergänzt. Dabei wird eine besondere Aufmerksamkeit auf die eigene Kultur des Teilnehmers gelegt, indem fremd Erscheinendes mit gewohntem Eigenen verglichen wird. Begleitend zu diesem für den Sprachunterricht üblichen Ablauf werden zusätzliche Informationen im Rahmen des Programms angeboten, die mit Hilfe von Analysen, Diskussionen, Rollenspielen und Si- 
mulationen erarbeitet werden sollen. Diese zusätzlichen Informationen beziehen sich auf interkulturelle Sachverhalte wie Verständnis der nationalen Kultur, Fremdes und Eigenes, kulturelle Stereotypen.

Das im dritten Teil dieser Arbeit ausgearbeitete Puzzle-Modell der interkulturellen Kompetenz bildet die theoretische Grundlage für das Programm zur Entwicklung der interkulturellen Kompetenz (PEIK). Dementsprechend sind die folgenden drei Lehrziele umzusetzen:

- Durch die Vermittlung von kulturspezifischen und kulturübergreifenden Kenntnissen wird die kognitive Kompetenz ausgebildet;

- durch die Entwicklung von kommunikativen und der interkulturellen Fähigkeiten wird die pragmatische Komponente aufgebaut;

- durch die Sensibilisierung gegenüber den Besonderheiten der interkulturellen Kommunikation wird die affektive Komponente der Kompetenz entwickelt.

Das Programm wurde im Fach „Deutsch als Fremdsprache“ für die weiterführenden Bildungseinrichtungen ausgearbeitet und ist als begleitende Maßnahme zu einem traditionellen nachschulischen Fremdsprachenunterricht gedacht. Dabei ist $\mathrm{zu}$ beachten, dass die Sprachlernenden keine Anfänger sind, da sie während der Schulausbildung mindestens sieben Jahre Deutschunterricht hatten und deshalb schon ein bestimmtes Sprachniveau besitzen. $^{36}$

Das Erlernen einer Fremdsprache in einer weiterführenden Bildungseinrichtung bedeutet auch, dass ein bestimmtes Ziel, wie z.B. die Entwicklung der fachbezogenen Kommunikationsfähigkeit, gestellt wird. Im Hinblick auf dieses Ziel wird der traditionelle Fremdsprachenunterricht entsprechend umgestaltet. Da aber die Idee des Programms auf der gegenseitigen Beeinflussung beider Komponenten (der sprachlichen und der interkulturellen) beruht, werden im traditionellen Unterricht auch neue Akzente gesetzt. Es wird immer ein Bezug zur interkulturellen Thematik bei den traditionellen Inhalten gesucht. Hierbei kommt die Vergleichsmethode zum Einsatz, die die Einbeziehung der eigenen Sprache und der eigenen Kultur der Teilnehmer voraussetzt.

Das Programm ist in vier Themenbereiche aufgeteilt (28 Sitzungen):

- interkulturelles Missverständnis

- Auto- und Heterostereotype

- kulturelle Selbstreflexion und Simulation

36 Das Sprachniveau der Lernenden wurde für das Programm nicht gemessen. Es ist aber zu beachten, dass dieses Niveau bei den Teilnehmern sehr unterschiedlich ist. 
- Sprachübungen und Landeskunde

Zur Einführung in das Programm werden einleitende Informationen angeboten, die die Ziele und zentralen Inhalte des Programms erklären. Als Abschluss und Zusammenfassung werden die interkulturellen Thesen zur Diskussion gestellt.

Die Frage nach der Sprache, in der die Inhalte des Programms im Unterricht eingebracht werden sollten, wurde folgendermaßen umgesetzt: Am Anfang des Programmablaufs, der auch der Anfang des Deutschunterrichts an der Hochschule für die Teilnehmer bedeutet, wird als Vermittlungssprache die Muttersprache der Teilnehmer ausgewählt. Im weiteren Verlauf des Programms kommt in einigen Teilen, wie z.B. in den Rollenspielen, auch Deutsch als Kommunikationssprache zum Einsatz. Die Muttersprache wird aus dem Unterricht nicht verdrängt, so dass bei schwierigen Inhalten die Teilnehmer auch auf ihre Muttersprache zurückgreifen dürfen. Diese Festlegung ergab sich aus folgenden Gründen:

- Am Anfang des Programmablaufs fangen die Teilnehmer ihr Studium an der Hochschule an. Obwohl sie keine Lernanfänger in der Sache Fremdsprachenlernen sind (Deutsch wurde als Pflichtfach in jeder Mittelschule vermittelt), ist damit zu rechnen, dass das Sprachniveau der Teilnehmer unterschiedlich ist. Im Laufe des Programms werden auch Sprachkenntnisse angeeignet, sodass davon auszugehen ist, dass die Sprachkompetenzen der Teilnehmer zum Ende des Programmsablaufs hin positive Veränderungen aufweisen.

- Die Muttersprache als Kommunikationssprache wurde auch ausgewählt, um den Start in die unbekannten interkulturellen Inhalte zu erleichtern. Die Vorträge und die Aufgaben können besser verstanden werden, auch die Teilnahme an den Diskussionen und die Analyse der Fälle wird erleichtert. Die relativ komplizierten Inhalte des Programms können freier und genauer besprochen werden.

Da das Programm sich nach dem Puzzle-Modell der interkulturellen Kompetenz richtet, die aus drei Komponenten besteht, ergeben sich folgende Aufgaben, die mit Hilfe des Programms zu lösen sind.

\begin{tabular}{|l|l|}
\hline $\begin{array}{l}\text { Bestandteile des Lernziels ,in- } \\
\text { terkulturelle Kompetenz“، }\end{array}$ & Aufgabe des Programms \\
\hline Kenntnisse & $\begin{array}{l}\text { Die Vermittlung von kulturspezifischen Kenntnissen } \\
\text { über die deutsche Kultur parallel zu dem ,reinen“ } \\
\text { Fremdsprachenunterricht, wodurch eine Ausdifferenzie- } \\
\text { rung des Auslandsbildes durch zusätzliche Informatio- } \\
\text { nen vorgenommen wird. }\end{array}$ \\
\hline
\end{tabular}




\begin{tabular}{|c|c|}
\hline & $\begin{array}{l}\text { Die Vermittlung kulturübergreifender Kenntnisse, wo- } \\
\text { durch das interkulturelle Bewusstsein zum Verhältnis } \\
\text { zwischen dem Eigenen und dem Fremden entwickelt } \\
\text { wird. }\end{array}$ \\
\hline Interkulturelle Sensibilität & $\begin{array}{l}\text { Die Entwicklung kultureller Sensibilität, indem } \\
\text { - positive Emotionen für die fremde Kultur ge- } \\
\text { weckt werden, } \\
\text { - bewusst mit den eigenen Emotionen in der inter- } \\
\text { kulturellen Situation umgegangen wird, } \\
\text { - Motivation für die gemeinsame Verständigung } \\
\text { entwickelt wird. }\end{array}$ \\
\hline $\begin{array}{l}\text { Interkulturelle Fähigkeiten und } \\
\text { Fertigkeiten }\end{array}$ & $\begin{array}{l}\text { Die Entwicklung der interkulturell wichtigen Fähigkei- } \\
\text { ten und Fertigkeiten, und zwar: } \\
\text { - Kommunikationsfähigkeit } \\
\text { - Fähigkeit zur Selbstreflexion } \\
\text { - Fähigkeit zur konstruktiven Auseinandersetzung } \\
\text { mit dem Eigenen und dem Fremden } \\
\text { - Fähigkeit zur Vermeidung und Klärung von } \\
\text { Missverständnissen } \\
\text { - Fähigkeit zum Umgang mit vorgefassten Mei- } \\
\text { nungen (Stereotypenkompetenz) }\end{array}$ \\
\hline
\end{tabular}

Bei der Festlegung einzelner Aufgaben ist zu beachten, dass sie unterschiedliche Erfolgsaussichten haben. Es ist davon auszugehen, dass die Aneignung der Kenntnisse schneller und unkomplizierter verlaufen wird, als die Arbeit an zwei anderen Aufgabengruppen. Allein aus dem Grund, dass die Entwicklung von Einstellungen ein lebenslanger Prozess ist und innerhalb eines kurzen Zeitrahmens nicht abgeschlossen werden kann, wird die Entwicklung der interkulturellen Sensibilität schwieriger sein. Ob die Entwicklung der Fähigkeiten und Fertigkeiten ,ausschlaggebend“ für das gesamte Lernergebnis sein wird, hängt vor allem von der Persönlichkeit und der interkulturellen Erfahrung jedes einzelnen Teilnehmers ab.

Es ist wichtig, die einzelnen Aufgaben des Programms zu bestimmen, weil die Auswahl der Inhalte und Methoden davon abhängig ist. Jede Arbeitsmethode zielt aber darauf ab, alle drei Aufgabengruppen zu beachten. Dazu ein Beispiel: Bei der Bearbeitung des Themas „Andere Länder in unseren Köpfen“ sind die Aufgaben aus allen drei Bereichen präsent: kognitive Aufgaben sind, Wissen über die kulturellen Erscheinungen zu sammeln und kulturelle Stereotype bewusst zu machen, die affektiven Aufgaben sind, Sensibilität gegen- 
über den vorgefassten Meinungen zu entwickeln und die emotionale Einstellung zu den eigenen Bildern zu reflektieren, die handlungsorientierte Aufgabe ist, Strategien zum Umgang mit Stereotypen zu erarbeiten, die jedem Einzelnen erlauben, den Einfluss der durch eine dritte Person erworbenen Meinung über die fremde Kultur zu reduzieren.

\subsection{Inhalte des Programms und Arbeitsmethodik}

Fremdsprachenunterricht ist vor allem Erwerb einer Fremdsprache. Die interkulturelle Seite des Unterrichts kann und darf davon nicht getrennt werden. Durch die Sprache und in der Sprache wird die Kultur erlernt, aus diesem Grund sind interkulturelle Inhalte schon teilweise durch die Bedingungen des Unterrichts ${ }^{37}$ vorprogrammiert, aber eben nur teilweise. Obwohl im Unterrichtsprogramm schon Hinweise auf die Notwendigkeit der Einbeziehung von z.B. landeskundlichen Informationen zu finden sind, sind diese Inhalte immer noch entfernt von denen, die die Teilnehmer auf die Situation eines interkulturellen Kontaktes vorbereiten können. In diesem Fall kommt es darauf an, unter welchem Aspekt die Arbeit an diesen Unterrichtsinhalten erfolgt.

Im Folgenden wird ein Überblick über die Themen (insgesamt 24) zur Umsetzung des Programms PEIK gegeben: Die Themen und die neuen Begriffe werden in dem nächsten Kapitel erklärt.

\begin{tabular}{|rl|}
\hline 1. & $\begin{array}{l}\text { Einführung: Erklärung der Programmziele, Definition der Begriffe Interkulturel- } \\
\text { le Kompetenz und Kultur, Eisbergmodell der Kultur }\end{array}$ \\
\hline 2. & $\begin{array}{l}\text { Fall: „Deutsch-brasilianisches Gespräch in der Firma“. Kulturassimilator-Metho- } \\
\text { de }\end{array}$ \\
\hline 3. & Fall: „Private“ Kulturassimilatormethode \\
\hline 4. & Referat: Kulturtheorien von Holl, Hofstede \\
\hline 5. & Fall: „Polizei“. Methode des offenen Falls \\
\hline 6. & Fall: „Der russische Gast“. Methode des offenen Falls \\
\hline 7. & Fallbeispiel aus den interkulturellen Erfahrungen des Lernenden. Methode: Ana- \\
& lyse des kritischen Ereignisses. \\
\hline 8. & Fallbeispiel aus den interkulturellen Erfahrungen des Lernenden. Methode des \\
& offenen Falls (zwei Sitzungen) \\
\hline 9. & Checkliste zur Erfassung der kulturellen Identität \\
\hline 10. & Satzergänzungsübung zur kulturellen Selbstbefragung \\
\hline 11. & Fragebogen zur Erhebung von Kulturkontrasterfahrungen \\
\hline 12. & Kulturelle Simulation \\
\hline & Fragebogen „Deutschland und Deutsche“ \\
\hline & Einführung ins Thema „Nationale Stereotype“. Diskussionsrunde „Deutschland \\
\hline
\end{tabular}

37 Jeder gesteuerte Unterrichtsprozess läuft unter bestimmten Bedingungen wie Ort, Zeit, Ziel, Zielgruppe, Leitziel der Unterrichtsanstalt, Lehrmaterial etc. ab. Näheres zu den Bedingungen im Kapitel 6.2 


\begin{tabular}{|l|}
\hline und Deutsche“ \\
\hline 13. Arbeit am Thema „Andere Länder in unseren Köpfen“ \\
\hline 14. Unterrichtseinheit „Typisch Russisch?“ \\
\hline 15. Ein studentischer Vortrag zum Thema „Einheit der Verschiedenen“ über die \\
Volksgruppen, Dialekte und Ausländer in Deutschland. Meinungsaustausch \\
\hline 16. Spielstunde „Konferenz der Tiere“ \\
\hline 17. Unterrichtssequenz „Spaghetti für zwei“ (zwei Sitzungen) \\
\hline 18. Spielstunde „Dil Dominoso“ \\
\hline 19. Unterrichtseinheit „Kulturelle Regeln und Tabus in Minidialogen“ \\
\hline 20. Unterrichtsstunde „Körpersprache“ \\
\hline 21. Vergleichsanalyse eines Märchens „,Der Wolf und die sieben Geißlein““ \\
\hline 22. Phraseologische Redewendungen. Wettbewerb \\
\hline 23. Diskussion zum Thema „Mein Deutschland““ \\
\hline 24. Arbeit an den Münchener Thesen zum interkulturellen Lernen \\
\hline
\end{tabular}

Für PEIK sind zwei Inhaltsbereiche zu beachten: die „traditionellen“ landeskundlichen Kenntnisse, die auch in den gängigen Lehrbüchern zu finden sind, bekommen eine andere Gewichtung und werden aus der neuen interkulturellen Perspektive heraus erlernt und erarbeitet. Die „neuen“ interkulturellen Inhalte bekommen einen Platz im Unterricht, den sie früher nie hatten. Sie werden durch unterschiedliche Arbeitsmethoden vermittelt.

Im Kapitel 4.2 werden die Programminhalte zusammen mit den Arbeitsmethoden vorgestellt, und zwar in vier methodischen Blocks:

1. Fallbeispiele und kritische Ereignisse

2. Übungen zu Stereotypen bzw. vorgefassten Bildern

3. Übungen und Tests zur kulturellen Selbstreflexion

4. Landeskunde und Sprachübungen

Die Auswahl dieser vier Blocks wurde zum Teil durch Themen der interkulturellen Didaktik beeinflusst. So unterscheidet Flechsig (2001) acht Trainingsmethoden: Fallmethode, Simulation, Kulturerkundung, Lernprojekt, Kleingruppen-Lerngespräche, Lern-Netzwerke, Werkstattseminar und „Übungen“ (vgl. 61). Dabei setzt er fünf Lerninhalte fest, die teilweise mit den Methoden übereinstimmen: Fallmethode, Simulationen, kulturelle Selbsterfahrungen und kulturelle Selbstreflexion und Verfremdungsübungen. In Anlehnung an Flechsig wurden in PEIK drei Gruppen interkultureller Inhalte übernommen, da sie die Beziehung zwischen dem Eigenen und dem Fremden gut darstellen, reflektieren und erleben lassen. Das sind Testfälle, Übungen zur kulturellen Selbstreflexion und Simulationsspiele. Alle drei lassen sich gut in den Kontext des Fremdsprachenunterrichts eingliedern. Außerdem wurden Übungen zum Umgang mit Stereotypen und vorgefassten Meinungen ins Programm aufgenommen. Das erwies sich aus folgendem Grunde als notwendig: Wie 
neurobiologische Untersuchungen festgestellt haben, ist die Bildung von Stereotypen ein ganz natürlicher Gehirnprozess (vgl. Keller 1991, 2007, Flechsig 2001). Die Stereotype und die vorgefassten Meinungen über fremde Völker werden hauptsächlich durch das in der eigenen Kultur erworbene Wissen vermittelt. Ohne reflektiert zu sein, werden sie im Fremdsprachunterricht eine Bestätigung und eine Verfestigung finden. Die fremde Kultur wird hauptsächlich nicht „live“ erlebt, sondern durch eine dritte Seite mitgeteilt, also durch ein Lehrbuch, durch Lehrmaterialien, durch die Persönlichkeit des Lehrers. Obwohl die Lehrbücher zur Zeit darauf orientiert sind, keine stereotypisierten Inhalte zu vermitteln und „interkulturelles Lernen“ immer öfter zum Bestandteil der Fortbildungen wird, kann man nicht ausschließen, dass die dritte Seite selbst durch vorgefasste Meinungen vorbelastet ist und sie weitergeben wird. Außerdem neigt der Sprachlernende wie jeder andere Mensch dazu, die Bestätigung seiner Stereotype zu suchen, damit er neue Informationen über die fremde Kultur in ein System einordnen kann. Aus diesen Überlegungen wurden die Übungen zum Umgang mit Stereotypen und vorgefassten Meinungen in PEIK eingegliedert.

Die Auswahl der Programminhalte und Arbeitsmethoden sind den Anforderungen der Gruppe angepasst. Es ist davon auszugehen, dass eine relativ homogene Gruppe an dem Experiment teilnimmt. Es sind junge Leute im Alter von 18-19 Jahren, die auch das gleiche Bildungsniveau haben, und zwar die abgeschlossene Schulausbildung, was im Deutschen dem Abitur gleichgesetzt werden kann. Das bedeutet, dass die Teilnehmer bestimmte Fähigkeiten zur Analyse und zur Reflexion aus dem schulischen Unterricht schon mitbringen. Die Aufgaben dürfen aber nicht mit Schwierigkeiten überlastet sein, weil es um Studierende geht, die noch keine universitäre Ausbildung und keine Erfahrungen im beruflichen Leben haben.

Zusammenfassend lassen sich drei Prinzipien, die bei der Auswahl der Programminhalte und deren Arbeitsmethoden berücksichtigt wurden, benennen:

- Inhalte und Methoden befinden sich in Übereinstimmung mit den Zielen und Aufgaben des PEIK;

- Die meisten Programminhalte und Arbeitsmethoden sind der interkulturellen Didaktik entliehen und für den Deutsch-als-Fremdsprache-Unterricht adaptiert;

- Die Inhalte und Arbeitsmethoden sind auf die Anforderungen der Programmteilnehmer angepasst. 


\subsubsection{Fallbeispiele:Arbeitsmethodik und Beispiele aus dem Programm}

\subsubsection{Ziele und Inhalte}

Fallbeispiele bilden den Grundstein der meisten interkulturellen Trainingsmaßnahmen. Unterschiedlich sind jeweils aber die Arbeitsmethoden, mit denen diese Fallbeispiele behandelt werden. In den folgenden Kapiteln wird vorgestellt, was unter Fallbeispielen oder kritischen Ereignissen gemeint ist, welche Varianten der Arbeitsmethoden mit ihnen Anwendung in der Praxis finden. Es werden auch einige Beispiele kritischer Ereignisse aus dem Programm vorgestellt und die ausgewählten Arbeitsmethoden begründet.

„Unter einem Fall versteht man ein zeitlich und räumlich zumeist eng begrenztes Ereignis, an dem zwei oder mehrere Personen aus mindestens zwei verschiedenen Ländern beteiligt sind, bzw. die sich durch mindestens zwei unterschiedliche kulturelle Orientierungen auszeichnen. Es kann sich um problematische, konflikthafte oder auch erfreuliche Ereignisse handeln“ (Flechsig 2001, S. 87).

In dieser Arbeit wird mit dem Begriff „Fallbeispiele“ gearbeitet. In den unterschiedlichen Quellen sind aber auch andere Namen zu finden, z.B. kritische Ereignisse, critical incident, Kulturassimilator etc. Es handelt sich dabei um die gleichen Inhalte, aber um unterschiedliche Arbeitsweisen. Was stellt eigentlich ein kritisches Ereignis dar? Fast jeder Mensch, der schon einen Auslandsaufenthalt erlebt oder mit Vertretern anderer Kulturen im Inland kommuniziert hat, hat eine Situation eines Missverständnisses erlebt, wobei er das Verhalten oder die Worte des Angehörigen der anderen Kultur „unverständlich“ fand. Wenn die Gründe dafür auf den kulturellen Hintergrund des Gegenübers zurückzuführen sind, handelt es sich um ein interkulturelles Missverständnis. Diese Gründe dienen als Ausgangsmaterial für die Analyse des kritischen Ereignisses.

Jedem kritischen Ereignis liegt also ein tatsächlich geschehenes Missverständnis zugrunde, welches für die beteiligten Kulturen charakteristisch ist. Häufig sich wiederholende interkulturelle Zwischenfälle sind ideales Material für ein kritisches Ereignis. Bei der Auswahl der kritischen Ereignisse ist es wichtig, dass sie typische Situationen darstellen. Die Situationen müssen im Idealfall kurz geschildert werden.

Nach Lacey und Trombridge (1995) werden bei der Bearbeitung kritischer Ereignisse folgende Lernziele verfolgt:

1. Probleme zu identifizieren, die wenigstens teilweise auf kulturellen Unterschieden beruhen; 
2. alternative Vorgehensweisen und Strategien zu entwickeln;

3. Entscheidungen zu treffen, die unterschiedliche kulturelle Perspektiven und Konsequenzen dieser Entscheidungen berücksichtigen;

4. Aufmerksamkeit und Anerkennung für kulturelle Unterschiede und für die Komplexität typischer Probleme zu sichern, welche die Lernenden wahrscheinlich in interkulturellen Situationen im Ausland und im Inland erfahren werden (zitiert nach Flechsig 2001, S. 87).

\subsubsection{Didaktisch-methodische Umsetzung}

Im PEIK werden fünf Fälle präsentiert. Es geht dabei um interkulturelle Missverständnisse zwischen Vertretern der deutschen und einer anderen Kultur. Es wird bewusst darauf verzichtet, ausschließlich Fälle auszuwählen, die ein interkulturelles Missverständnis zwischen den Vertretern der russischen und der deutschen Kulturen präsentieren. In diesem Fall werden die Lernenden unbewusst vorhandene Schemata aufgreifen, die vorgegebenen Fälle werden sie gewiss bestätigen. Solch stereotypes Denken aufzudecken scheint leichter zu sein, wenn man mit fremden Nationen arbeitet. Es werden aber im Programm zwei „besondere" Fälle eingeschlossen, durch welche die Teilnehmer des Experiments eine persönliche Beziehung aufbauen: ein deutsch-russischer Fall und ein Fall aus dem Polizeialltag.

Wie schon oben erwähnt, sind die Arbeitsmethoden für kritische Ereignisse unterschiedlich. Am bekanntesten ist die Kultur-Assimilator-Methode (Cultural Assimilator). Sie wird oft als grundlegende Trainingsmethode in den kulturspezifischen Trainings zur Vorbereitung eines Auslandsaufenthalts verwendet. Im deutschsprachigen Raum wurde eine Reihe von Cultural-Assimilator-Trainings veröffentlicht, als deren Co-Verfasser jeweils Prof. Dr. Thomas (z.B. Yoosefi und Thomas 2008) auftritt.

Die Arbeitsmethodik dieser Cultural Assimilator sieht folgendermaßen aus: Zu jedem Fall wird ein Verhalten beschrieben, und es werden mehrere Erklärungsmöglichkeiten angeboten. So beschreibt Bittner (o.J) weitere Vorgehensweise:

„Der Leser soll sich für eine davon entscheiden und die Rückmeldung zur gewählten Antwort einsehen. Wenn die Auswahl des Lesers nicht der Intention des Autors entspricht, soll er es noch einmal versuchen. Gegebenenfalls werden die zu vermittelnden Inhalte anschließend noch einmal zusammengefasst" (Bittner o.J., S. 2). 
Jeder der angebotenen Rückmeldungen liegen Kulturstandards zugrunde. Unter Kulturstandards werden die Werkzeuge verstanden, die im Prozess der Sozialisation erworben wurden und mit denen der Vertreter einer Kultur sein Verhalten regelt. Das Ziel der Cultural Assimilator besteht darin, die Lernenden gegenüber einer anderen kulturellen Prägung zu sensibilisieren, indem sie Wissen über die fremden Kulturstandards und möglichen Unterschiede vermittelt.

Im PEIK werden zwei kritische Ereignisse mit dieser Methode bearbeitet, und zwar „Deutsch-brasilianisches Gespräch in der Firma“" und „Privat“".

Fallbeispiel „Privat“

„Ein junger Amerikaner, der gerade seinen Universitätsabschluss hinter sich hat, arbeitet als Sachbearbeiter. Wegen einer Unterschrift geht er ins Büro seines deutschen Abteilungsleiters, der sich zufällig gerade im Vorzimmer bei seiner Sekretärin aufhält. Der Abteilungsleiter nimmt sein Anliegen auf und wechselt ein paar Worte mit ihm. Bei dieser Gelegenheit fragt er ihn, woher er käme, wie es ihm in Deutschland gefalle, wie lange er in Deutschland bleiben wolle, was er hier vorhätte. Da der amerikanische Mitarbeiter den Abteilungsleiter durchaus sympathisch findet, fragt er ihn, ob er am Abend nicht mit ihm zu einer Party kommen wolle. Dieser antwortet, dass das nicht möglich sei, weil er nach München fahren müsse. Als der Amerikaner daraufhin wissen will, was er dort zu tun habe, beendet der Abteilungsleiter die Unterhaltung, indem er kurz und knapp antwortet: ,Privat'. Der Amerikaner fühlt sich zurückgestoßen: Eben noch hatte er dem Abteilungsleiter bereitwillig Auskunft über sein Privatleben gegeben, da sich dieser ja offensichtlich mit ihm unterhalten wollte, und jetzt reagiert der Mann so abweisend.

Deutungen:

a) Der Abteilungsleiter fühlt sich von dem Angebot seines Untergebenen beleidigt, mit ihm zusammen zu einer Party zu gehen. Deshalb bricht er das Gespräch möglichst schnell ab.

b) Es gehört zu den Aufgaben eines deutschen Chefs, Interesse für neue Mitarbeiter zu zeigen. Dazu gehören auch Informationen aus dem Privatleben des Mitarbeiters. Da das Gespräch in Anwesenheit einer dritten Person stattgefunden hat, und zwar einer Sekretärin, hat der deutsche Chef seine „Pflicht" getan. In der Tat hatte er gar kein Interesse an dem Amerikaner. Nachdem die höflichen Fragen gestellt und beantwortet wurden, hält er das Gespräch für erfüllt und geht auf die weiteren Fragen des Amerikaners nicht ein.

c) In Deutschland sind Fragen zum Privatleben der Kollegen mit Vorsicht zu stellen, das gilt gegenüber dem Chef ganz besonders. Der Abteilungsleiter hatte das Gefühl, dass die angebrachte Distanz zwischen ihm und seinem Mitarbeiter sich zu sehr verringern könnte, was sich negativ auf das weitere Arbeitsverhältnis auswirken kann.

Erläuterungen:

a) Diese Antwort ist unwahrscheinlich. Nichts spricht dafür, dass der Abteilungsleiter durch das Angebot des jüngeren Kollegen beleidigt wurde. Suchen Sie eine andere Antwort. 
b) Es fällt schwer, diese Antwort zu verneinen, da diese Aussage sicherlich die Situation aus einem anderen Blickwinkel erhellt. Um miteinander gut am Arbeitsplatz auszukommen und den Arbeitsprozess zu beleben, versucht man Interesse an seinem Gesprächspartner zu zeigen. Trotzdem ist es für den deutschen Abteilungsleiter kein Muss, er ist nicht verpflichtet, solche Konversationen mit seinem Mitarbeiter zu führen.

c) Mit dieser Aussage haben Sie den Kern der Sache getroffen. „Chef-Mitarbeiter-Beziehungen" bewegen sich üblicherweise auf dem Distanzlevel von Bekannten. Und hier erhält die Anrede per ,Sie' eine wesentliche Bedeutung. Mit ihr wird die Aufgabenorientiertheit sichergestellt, weil verhindert werden soll, dass sich Privates und Berufliches vermischt. Bei einem Näherkommen würde sich die Distanz verringern und die Mitarbeiterführung wäre schwieriger, denn Freundlichkeit und Herzlichkeit sind im Privatleben angesiedelt und verpflichten zu einer Berücksichtigung persönlicher und emotionaler Belange. Das würde den Chef darin einschränken, seinen Mitarbeitern das sachlich Optimale abzuverlangen und den Mitarbeiter dazu ermutigen, weniger strikt die Erfordernisse der Rolle als vielmehr eigene (momentane) Befindlichkeiten im Auge zu haben" (Schroll-Mahl 2003, S. 150f).

Bei der Analyse jedes Falls ist es wichtig, dass die Diskussion über die möglichen Erklärungen in kleinen Gruppen verläuft und dass jede Gruppe sich zuerst auf eine Erklärung einigt, die ihren Mitgliedern besonders wahrscheinlich vorkommt. Die Entscheidung muss begründet werden. Im Weiteren werden die Rückmeldungen zu den ausgewählten Erklärungen gelesen, danach müssen sie aber auch reflektiert und ausdiskutiert werden. Die möglichen Denkmuster aus der ersten Diskussion (besonders wenn sie zu den unwahrscheinlichen Erklärungen geführt haben) sind aufzudecken. Es geht im Prinzip nicht nur darum, die fremden Kulturstandards kennenzulernen, sondern auch die eigene Einschätzung zu reflektieren.

In der Regel ist es auch so, dass keine eindeutige Erklärung zu jedem Fall existiert, sondern dass mehrere Erklärungsalternativen zutreffend sein können. Es wird dabei nur über die Wahrscheinlichkeit jeder Erklärung gesprochen. Diese Tatsache, dass ein Missverständnis nicht an einer Erklärung festgemacht werden kann, vermittelt den Lernenden, dass zu jeder nicht eindeutigen Situation zwischen Vertretern von zwei oder mehreren Kulturen verschiedene Ursachen, häufig auch solche nicht interkultureller Natur, zugrunde liegen können.

Als nächste Methode wird die Methode des offenen Falls eingesetzt. Hier werden die Fälle aus der Perspektive eines außenstehenden Beobachters erzählt. Die Aufgabe des Lerners besteht darin, den Fall aus den Perspektiven seiner Teilnehmer zu erzählen und im Weiteren eine Hypothese zu bilden, die die Gründe dieses Missverständnis erklärt. 
Fallbeispiel „Ein russischer Gast“

Eine russische Mitarbeiterin in einer deutschen Firma in Deutschland wird in eine andere Stadt versetzt. Da sie an ihrem früheren Arbeitsort Freunde gefunden hat, fährt sie ab und zu übers Wochenende zu diesen Freunden zu Besuch. Sie wird dabei jedoch das Gefühl nicht los, dass sie diesen Freunden lästig ist. Zwar wird sie immer herzlich begrüßt, und die Freunde sagen jedes Mal, wie sehr sie sich freuen, sie wiederzusehen, doch dann überlegen sie sich in ihrer Anwesenheit ungeniert, was jeder am Wochenende machen muss und tun will. Sie stellen daraufhin jeder für sich (teils mit und teils ohne ihren russischen Gast (willst du mitmachen?) das Programm zusammen. Oft haben sie noch etwas zu erledigen, tun das auch und lassen ihre russische Freundin dann allein. Sie kann ja einkaufen gehen oder etwas anderes machen - sie kennt sich ja aus in der Stadt. Ihr Spruch heißt: ,Fühl dich frei!' (Schroll-Mahl 2003, S.201).

Beim Versuch, den Fall aus der Perspektive eines Teilnehmers zu erzählen wird der Lernende mit der Notwendigkeit konfrontiert, sich in das Verhalten eines anderen Menschen hineinzudenken, dessen Worte und Verhalten durch plausible Gründe zu erklären. Dadurch werden vereinfachte Bewertungen wie „schlecht“ oder „gut“ vermieden, die kulturellen Orientierungen der Person, deren Perspektive aufgenommen wird, können entdeckt werden. Dabei ist selbst der Versuch wertvoll, sich von der Rolle des Vertreters seiner eigenen Kultur abzulösen, d.h. die Situation mit „fremden Augen“ zu interpretieren.

Offene Fälle können mit oder ohne Interpretationshilfen angeboten werden. Interpretationshilfen enthalten Hinweise auf die Normen, Wertvorstellungen und Verhaltensmuster, die dem Fall zugrunde liegen können. Als Interpretationshilfen können auch theoretische Befunde dienen, die auf Unterschiede zwischen den Kulturgemeinschaften hinweisen. Sie helfen auch bei der Bildung der Hypothesen zur Interpretation des Falls. Als Hilfe bei der Bearbeitung der Fälle mit dieser Methode werden Lernaufgaben gestellt.

Im PEIK werden vier offene Fälle angeboten: Im Weiteren werden der Arbeitsablauf und die Lernaufgaben vorgestellt, die jeden Fall begleitet haben.

- Einführende Worte (von dem Fall abhängig),

- Vorstellung des Falls,

- Diskussion über die ersten Gedanken und Gefühle, die nach der Vorstellung des Falls aufgetreten sind,

- Vorstellung des Falls aus der Perspektive seiner Teilnehmer (die Vorbereitungsarbeit wird in kleinen Gruppen durchgeführt und dann im Plenum vorgestellt),

- Ausarbeitung der gemeinsamen Interpretationshypothese im Plenum,

- Abschluss der Diskussion. (Besprechung der alternativen Verhaltensstrategien der Teilnehmer, wie wird der Fall abgeschlossen?)

Als Interpretationshilfe werden im Voraus die Kulturtheorien von Hall und Hall (1989) und 
Hofstede (1980) präsentiert. Diese Theorien bilden den theoretischen Teil des Programms. Sie werden von einem Lernenden (mit der Unterstützung des Lehrers) als fakultative Aufgabe vorbereitet und vorgetragen. Dieser Referent übernimmt dann bei der Aufstellung der Hypothesen die Rolle eines Experten.

Als Abschluss wird ein Fall mit der Methode Analyse kritischer Ereignisse bearbeitet. Dafür wird es den Lernenden angeboten, über persönlich erlebte Ereignisse nachzudenken und sich an die zu erinnern, die einen interkulturellen Hintergrund haben. Es ist davon auszugehen, dass nicht alle Lernenden schon einen Auslandsaufenthalt erlebt haben. Aus diesem Grund wird darauf hingewiesen, dass das zu erinnernde Ereignis auch im Inland geschehen sein könnte und dass der Gesprächspartner auch aus dem „nahen Ausland“ kommen oder ein Vertreter einer anderen Nation sein könnte, der in Russland lebt. ${ }^{38}$ Dabei muss es sich um eine Situation handeln, der ein Missverständnis oder ein Konflikt zugrunde liegt, aus der der Lernende etwas gelernt hat, woran er sich oft erinnert.

Bei der Analyse kritischer Ereignis muss der Lernende versuchen, die ausgewählte Situation möglichst genau zu beschreiben und dabei Bewertungen zu vermeiden. Für die Beschreibung der Situation wurde folgendes Formular angeboten, das den Lernenden hilft, die Situation in ihrem gesamten Kontext zusammenzufassen.

Formular zur Erfassung von kritischen Ereignissen:

1. Überschrift

2. Wo fand das Ereignis statt?

3. Wer war bei dem Ereignis in welcher Rolle anwesend?

4. Wann fand das Ereignis statt?

5. Welche Ereignisse, die möglicherweise von Einfluss waren, waren der Situation vorausgegangen?

6. Wie wurde die Situation eröffnet?

7. Worin bestand das "kritische Ereignis"?

8. Wie wurde die Situation abgeschlossen?

9. Was geschah danach?

10. Welche Erfahrungen haben Sie dabei gemacht? (vgl. Flechsig 2001, S. 95).

38 Nach dem Zerfall der Sowjetunion ist der Ausdruck „,nahes Ausland“ gängig. Damit sind die ehemaligen sowjetischen Republiken gemeint wie Weißrussland, Georgien, Kasachstan etc. Da in der Sowjetunion die Politik des Internationalismus propagiert wurde, sind viele Menschen aus diesen Republiken nach Russland gezogen, wo sie bis heute leben. Zur Multikulturalisation der Gesellschaft trägt auch die Tatsache bei, dass sich auf dem Territorium des heutigen Russland mehrere nationale Kreise befinden, das heißt mehrere unterschiedliche Völker leben. Auf dem Territorium des Irkutsker Verwaltungsgebiets befindet sich z.B. der autonome Kreis der Ust-Ordynsker Burjaten. 
Es ist auch wichtig, dass der Lernende bewusst auf Bewertungen des Verhaltens seines Kommunikationspartners verzichtet. Er muss versuchen, sich von der Situation zu distanzieren. Er darf zwar die eigenen Emotionen beschreiben, was auch eine Selbstreflexion braucht. Der Lernende muss aber auf die Feststellung der Emotionen des Partners verzichten, er darf lediglich seine eigenen Vermutungen über den emotionalen Zustand des Partners ausdrücken.

In der nächsten Arbeitsphase werden alle Beschreibungen ausgehängt, und durch Abstimmung wird ein kritisches Ereignis ausgewählt, das am stärksten das Interesse der Lernenden geweckt hat. Der Autor dieser Beschreibung kann anonym bleiben, kann sich aber auch in Laufe der weiteren Diskussion der Öffentlichkeit präsentieren. Im Weiteren wird dieser Fall mit der Methode des offenen Falls bearbeitet.

Der Sinn der Methode der Analyse kritischer Ereignis besteht darin, den Lernenden dazu zu bringen, sich selbstständig mit den eigenen Erfahrungen auseinandersetzen und sein eigenes Verhalten zu reflektieren. Durch die Distanzierung zum Geschehenen und zu den Emotionen gewinnt er die Chance, über das Verhalten des Partners nachzudenken, ohne dass es negativ bewertet wird.

In PEIK werden fünf Fälle präsentiert, die mit drei unterschiedlichen Arbeitsmethoden bearbeitet werden, und zwar der Kulturassimilator-Methode, der Methode des offenen Falls und der Methode der Analyse des kritischen Ereignisses. Die Fallbeispiele bilden eine der wichtigsten Elemente in jedem interkulturellen Training, weil sie leicht zu bearbeiten sind und auf lebensrealen Angelegenheiten basieren. Sie enthalten auch relativ standardisierte Erklärungen, die im Prinzip leicht in Handlungsanweisungen für bestimmte Lebenssituationen umzuwandeln sind. Bei der Arbeit mit Fallbeispielen besteht aber auch die Gefahr der Stereotypenbildung. Da die kritischen Situationen bei der Bearbeitung standardisiert werden, neigen die Lernenden dazu, bestimmte Verhaltensmuster den Vertretern einer anderen Kultur als starre Klischees zuzuordnen. Es bildet sich bei den Lernenden die Überzeugung, dass Vertreter einer anderen Kultur in einer bestimmten Situation unbedingt ein bestimmtes Verhaltensmuster einsetzen. Diese Meinung ist nicht unbedingt falsch, aber entspricht auch nicht zu hundert Prozent die Wahrheit. Deshalb ist es wichtig, in einem Programm nicht nur Fallbeispiele zu bearbeiten, sondern auch weitere Trainingsaspekte zu verfolgen wie Selbstreflexion und Arbeit mit Stereotypen. 


\subsubsection{Kulturelle Selbstreflexion: Arbeitsmethodik und Beispiele aus dem Programm}

\subsubsection{Ziele und Inhalte}

Selbstreflexion bildet das zweite Grundelement unseres Programms. Der Begriff „Selbsterfahrung", der eigentlich aus der philosophischen und psychologischen Literatur stammt, bezieht sich auf das Reflektieren über das Erleben und Verhalten der eigenen Person, des eigenen Selbst. Es kann sich dabei um eigene Emotionen, eigene Worte, eigenes Handeln, eigene Reaktionen handeln. Wenn wir aber über die kulturelle Selbsterfahrung sprechen, „so geht es nicht in erster Linie um persönliche Eigenschaften, Lebensweisen oder Verhaltensweisen (, den Charakter eines Menschen'), sondern um jene Lebensweisen, Verhaltensmerkmale und Eigenschaften von Menschen, die kulturspezifisch sind; es sind dies Verhaltensweisen und Einstellungen, Weltbilder und Menschenbilder, die sie mit Menschen teilen, die in gleichen kulturellen Kontexten aufgewachsen sind und von ihnen geprägt wurden“(Flechsig 2001, S. 106).

Bei der kulturellen Selbstreflexion geht es um das Erkennen des eigenen kulturellen „Ich“. Bei der Kommunikation mit Vertretern der eigenen Kultur erkennt der Mensch nicht, dass er eigentlich durch die eigene Kultur mit allen ihren Werten und Einstellungen beeinflusst ist. Ihm bleibt dabei verborgen, dass seine Familie, Umgebung, Schule, Medien eine kulturgeprägte Spur bei der Entwicklung der persönlichen Identität hinterlassen haben. Es wird ihm erst bewusst, wenn er mit Vertretern anderer Kulturkreise kommuniziert. Dann wird dem Menschen schnell klar, dass der fremdkulturelle Partner andere Vorstellungen und Einstellungen hat und dass ein gemeinsamer kultureller Hintergrund für die Kommunikation fehlt. Bei oberflächlichen interkulturellen Begegnungen bleibt einem Menschen aber oft das Bewusstsein „erspart“, dass er selbst nicht „,neutral“ in seinen Einschätzungen und Handlungen ist. Nur durch das Nachdenken über solche Kontrasterfahrungen und über die eigene Rolle darin kann die kulturelle Selbstreflexion erreicht werden.

Bei der Thema „Selbstreflexion“ wird über solche Phänomene wie „kulturelle Identität“ gesprochen. Uhle (2006) definiert diesen Begriff folgendermaßen:

„Unter kultureller Identität wird die Gesamtheit der kulturell geprägten Werte samt der daraus resultierenden Weltsichten und Denkweisen sowie der ebenfalls kulturell geprägten Verhaltens- und Lebensweisen verstanden, die das Eigenbild einer Kulturgemeinschaft namentlich einer Nation - prägen. Die so verstandene kulturelle Identität wird sowohl durch Elemente der Zugehörigkeit zu einem (übergeordneten) Kulturkreis, als auch durch 
Elemente der Zugehörigkeit zu der individuellen Kultur der betreffenden Gemeinschaft bestimmt“" (zitiert nach Scholz 2008, S. 35).

Im Laufe der kulturellen Selbsterfahrung wird dem Menschen die eigene kulturelle Identität bewusst. Man muss aber immer darauf hinweisen, dass kulturelle Identität keine starre Erscheinung ist. Die moderne Gesellschaft ist längst nicht mehr homogen und schließt mehrere Subkulturen ein. Der moderne Mensch vertritt nicht nur die eigene nationale Kultur, sondern er ist auch ein Teil von mehreren anderen Subkulturen, die sowohl ähnliche als auch gegensätzliche Werte präsentieren. Deshalb wird zur Zeit über das Aushandeln der eigenen Identität gesprochen, wobei der Mensch sich nicht nur mit einer Nation identifiziert, sondern auch bewusst wahrnimmt, dass er sich auch anderen Bezugssystemen zugehörig fühlt. Bei dem Aushandeln der kulturellen Identität kann darüber gesprochen werden, welche Werte die Person für sich als Norm akzeptiert.

Die Aufgabe der Selbstreflexion ist es, ein Bewusstsein sowohl für die eigenen Werte und Einstellungen als auch für ihre Entstehungsgeschichte zu entwickeln, d.h. den Einfluss der kulturellen Umgebung auf die Entwicklung des eigenen Selbstbildes zu erkennen. Die Fähigkeit zur Selbstreflexion muss im Weiteren den Lernenden helfen, bei interkulturellen Kontakten ihre eigene kulturelle Position wahrzunehmen. Dieses Bewusstsein sollte den Lernenden erlauben, sich von der eigenen Position und den eigenen Emotionen zu distanzieren. In extremen Situationen, z.B. in der Situation des interkulturellen Missverständnisses, befähigt es dazu, keine vorläufige Beurteilung zu treffen, sondern mit „klarem Kopf“ nach einer Lösung zu suchen.

Bei der kulturellen Selbstreflexion geht es um die Beziehung zwischen dem „Fremden“ und dem „Eigenen“, wobei das „Eigene“ in den Vordergrund rückt.

\subsubsection{Didaktisch-methodische Umsetzung}

Im PEIK wurde bereits im ersten Teil des Programms mit der kulturellen Selbstreflexion begonnen. Obwohl die Methode ,Analyse des kritischen Ereignisses “ offiziell in den Fallstudien angewandt wird, stellt sie nichts anderes als eine Selbstreflexion über kulturelle Kontrasterfahrungen vor. Durch das Erinnern einer interkulturellen Begegnung werden die Lernenden zum einen dazu motiviert zu versuchen, das Verhalten des fremdkulturellen Partners zu verstehen. Sie werden außerdem dazu angeregt, über die eigenen Gefühle und eigenes Verhalten in dieser Situation nachzudenken. Durch die kulturellen Konstrukte, die 
dem Lernenden dabei fremd und ungewöhnlich vorkommen, wird er aufgefordert zu verstehen, was für ihn/sie selbst in der angegebenen Situation normal ist, welche eigenen kulturellen Konstrukte er als tauglich ansieht.

In diesem Zusammenhang dient der letzte Fall, der mit der Methode „Analyse des kritischen Ereignisses“ bearbeitet wurde, als Bindeglied zum zweiten Teil des PEIK, nämlich „Selbstreflexion“. Im zweiten Teil werden folgende Aufgaben bearbeitet: Checkliste zur Erfassung der kulturellen Identität, Satzergänzungsübung zur kulturellen Selbstbefragung, Fragebogen zur Erhebung von Kulturkontrasterfahrungen und kulturelle Simulation.

Die Checkliste zur Erfassung der kulturellen Identität besteht aus zwei Teilen. Im ersten Teil werden die Lernenden aufgefordert, den Satz „Ich bin ...“ fünfmal zu ergänzen. Im Teil zwei füllen die Lernenden folgende Tabelle aus:

\begin{tabular}{|l|l|l|}
\hline Ich fühle mich als & + & $\%$ \\
\hline - Weltbürger & & \\
\hline - Russe, Burjate, Jakute etc. & & \\
\hline - Sibirjake & & \\
\hline - Kursant/ Student des Militärinstituts & & \\
\hline - Mitarbeiter des Innenministeriums & & \\
\hline - orthodoxer Christ, Buddhist, Atheist etc. & & \\
\hline - Mitglied einer politischen Partei & & \\
\hline - Sportler (Musiker etc.) & & \\
\hline - Mitglied eines Vereins & & \\
\hline - einer von unserer Clique & & \\
\hline - Sohn/Tochter & & \\
\hline .... & & \\
\hline .... & & \\
\hline Ich fühle mich in erster Linie als eigenständige Person. & & \\
\hline
\end{tabular}

Beim Ausfüllen der oben angegebenen Tabelle muss wie folgt vorgegangen werden:

1. Bestimmen Sie, zu welchen unten angegebenen Gruppen Sie sich zugehörig fühlen. Kreuzen Sie diese Gruppen in der zweiten Spalte an.

2. Wenn eine für Sie wichtige Bezugsgruppe fehlt, schreiben Sie sie in die freien Zeilen.

3. Überlegen Sie, welche Anteile jede Komponente hat. Verteilen Sie in der dritten 
Spalte die prozentualen Anteile, es müssen insgesamt 100\% ergeben.

Nachdem die beiden Aufgaben zur kulturellen Identität erfüllt sind, werden zwei Expertengruppen aus allen Teilnehmern gebildet. Jede Gruppe bearbeitet die Ergebnisse einer Aufgabe und präsentiert sie auf einer Folie oder einem Plakat. Anschließend werden die Ergebnisse zusammengefasst und die eigene Meinung dazu ausgedrückt.

Der Sinn dieser Aufgaben ist es, während der Selbstbefragung die Teilnehmer zum Nachdenken über kulturelle Gruppen anzuregen, denen sie sich zugehörig fühlen. Es wird festgestellt, dass sich ein Mensch innerhalb mehrerer kultureller Gruppen bewegt und dass eine scheinbar „homogene“ Gruppe in der Tat relativ bunt aus allen kulturellen Richtungen gemischt ist. Es geht auch darum, die Teilnehmer darauf aufmerksam zu machen, dass der Anteil der nationalen Kultur nicht als überwiegend präsentiert ist.

Folgende Fragen können als Anregung bei der anschließenden Diskussion gestellt werden:

- Sie denken, Sie sind ein guter Sibirjak/ Weltbürger/ Sohn etc. Wie erkennen Sie das?

- Was ist Ihnen als Sibirjak/ Weltbürger/ Sohn wichtig?

- Kann es vorkommen, dass die unterschiedlichen Anteile von Ihnen im Konflikt geraten? z.B. Sie empfinden etwas als Weltbürger für richtig, der orthodoxe Glaube sieht es aber als falsch an. Können Sie eigene Beispiele nennen?

- Welche Auswirkungen können die festgestellte Tatsachen auf die Kommunikation mit Vertretern einer anderen nationalen Kultur haben?

Bei der Satzergänzungsübung zur kulturellen Selbstbefragung geht es sowohl um die Erfassung der kulturellen Identität als auch um die eigener Werte, die dem Teilnehmer besonders wichtig sind. Die Übung ist als Lückentext aufgebaut. Die Teilnehmer füllen die Lücken mit Wörtern aus, die ihnen möglichst spontan einfallen. Im Weiteren werden die Ergebnisse zusammengefasst und präsentiert. In der abschließen Diskussion werden sowohl die eigenen Gedanken der Teilnehmer bei dem Ausfüllen der Lücken als auch die ans Licht gekommenen Werte besprochen. Hier ist die Satzergänzungsübung zur kulturellen Selbstbefragung:

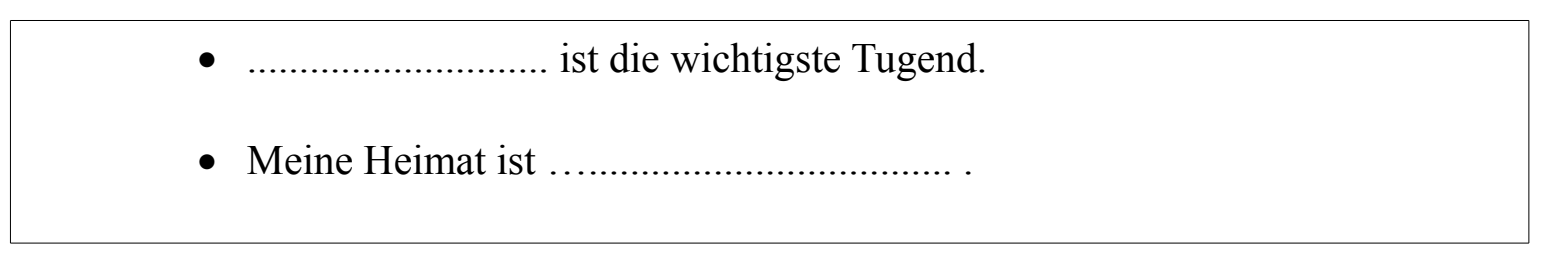


- Ich bin Bürger meines Landes zu sein.

- Ich fühle mich zugehörig.

- Ich engagiere mich für

Beim Fragebogen zur Erhebung von Kulturkontrasterfahrungen geht es vor allem um die kulturelle Orientierung oder um Kulturstandards. In solchen Fragebögen werden zu einem wichtigen kulturellen Konzept Fragen gestellt bzw. Aussagen getroffen wie z.B. zum Umgang mit Zeit oder Raum. Dazu werden zwei oder mehrere Antwortalternativen angeboten, die unterschiedliche kulturellen Orientierungen widerspiegeln. Bei der Auswahl passender Beispiele, die einer kulturellen Norm entsprechen, geraten sofort die Alternativen in das Blickfeld des Teilnehmers. Dadurch wird ihm nicht nur die eigene kulturelle Norm bewusst, sondern auch dass diese kulturellen Standards für Vertreter der anderen Kultur anders sein können. Hier sind einige Beispiele aus dem Fragebogen:

1. Welches der unten angegebenen Sprichwörter passt zu mir:

- $\quad$ Zeit ist Geld

- $\quad$ Kommt Zeit, kommt Rat

2. Wenn ein Fremder mich über mein Land fragt, antworte ich ...

- eher ehrlich

- $\quad$ eher höflich

3. Körperkontakte sind ...

- $\quad$ zu vermeiden

- aufzunehmen

In den oben angeführten Beispielen geht es um drei Kulturstandards, und zwar Umgang mit der Zeit, Direktheit der Kommunikation und Angemessenheit taktiler Kontakte.

Wenn bei der Bearbeitung der Fallbeispiele über die „fremden“ kulturellen Orientierungen gesprochen wird, werden die eigenen kulturellen Orientierungen beim Ausfüllen dieses Fragebogens zum Erkenntnisobjekt. Bei der Bearbeitung der Fälle bleibt öfters die eigene kulturelle Position im Schatten, da sie als „,normales“ Benehmen oder „normale“ Reaktion bezeichnet wird. Die Möglichkeit, zwischen zwei Alternativen auszuwählen, verwirft den Begriff „normal“, sie polarisiert die möglichen Verhaltensmuster und zwingt den Teilnehmer, sich an einem der Pole zu positionieren. Das Bewusstsein über die Existenz von zwei Polen soll den Teilnehmer zur Sensibilität gegenüber dem Fremden führen. Die eigenen kulturellen Muster werden bewusst, „um zu wissen, womit ein neuralgischer Punkt bei an- 
deren getroffen wird und sich selbst rechtzeitig, also vor Erreichen der Schmerzgrenze des anderen, Einhalt zu gebieten“ (Schroll-Mahl 2003, S. 25f).

In dem Fragebogen zur Erhebung von Kulturkontrasterfahrungen werden zwölf Fragen oder Aussagen angeboten, die die wichtigsten kulturellen Orientierungen widerspiegeln: Umgang mit Zeit und Raum, taktile Kontakte, schwache vs. starke Kontexte als Kommunikationsstil $^{39}$, nonverbale Kommunikation, starke vs. schwache Unsicherheitsvermeidung. Nach Ausfüllen des Fragebogens werden die Ergebnisse auf einer Folie zusammengefasst und präsentiert. Anschließend wird eine Diskussion mit den folgenden Leitfragen durchgeführt:

- Welche Übereinstimmungen/Differenzen sind festzustellen? Wie können die extremen Differenzen (wenn solche zu erkennen sind) innerhalb einer relativ kulturell einheitlichen Gruppe erklärt werden?

- Wieweit lässt sich annehmen, dass die Übereinstimmungen, die wir in unserer Gruppe festgestellt haben, für die meisten Bürger Russlands oder die meisten Menschen gelten?.

- Welche Situationen sind vorstellbar, in denen die am häufigsten angekreuzten Orientierungen zur Geltung kommen? Welche Konsequenzen kann das für das Benehmen eines Menschen haben?

- Welche Situationen sind vorstellbar, in denen Menschen mit gegensätzlichen kulturellen Orientierungen einander kontaktieren? Welche Konflikte sind voraussehbar?

Ein weiteres Instrument, das im PEIK für die Selbstreflexion benutzt wird, ist ein Simulationsspiel. Simulation stellt ein Rollenspiel dar, in dem eine Interaktion zwischen den Vertretern von zwei oder mehreren Kulturen nachgestellt wird. Zwischen einem kulturspezifischen und einem kulturunspezifischen Simulationsspiel wurde im PEIK zugunsten des letzteren entschieden. In dieser Simulation wird eine Kommunikation zwischen zwei Gruppen nachgespielt, die zwei fiktive Kulturen vertreten. Die Tatsache, dass keine real existierende Kultur nachgespielt wird, bezweckt den folgenden Effekt: die Teilnehmer orientieren sich an keiner Kultur, d.h. der Zugriff auf nationale Stereotypen wird vermieden.

39 Der Begriff schwacher und starker Kontext (high/ low context) wurde von Hall eingeführt (vgl. Hall/Hall 1989). Bei der Kommunikation in den Kulturen mit dem starken Kontext wird vorausgesetzt, dass die meisten Informationen dem Gesprächspartner schon bekannt sind und dass die Erwähnung von Details überflüssig ist. Der nonverbalen Kommunikation und dem Beachten der Umstände wird dabei eine große Bedeutung beigemessen. Das Beispiel für die Kultur mit dem starken Kontext ist Japan. Die Kommunikation in den Kulturen mit dem schwachen Kontext ist direkter und enthält mehr Details, so z.B. in den USA. 
Die Simulationsspiele haben zwei unbestrittene Vorteile: sie sind verhaltensorientiert und sie appellieren sehr stark an die Emotionen der Teilnehmer. Nach den Spielregeln treten zwei fiktive Kulturen in die Kommunikation mit dem Ziel, eine gemeinsame Aufgabe zu lösen. Aufgrund der vorgegebenen Verhaltensweisen sind die interkulturellen Konflikte vorprogrammiert. Die Spieler sind gezwungen, einerseits sich kulturgebunden zu verhalten, andererseits die entstehenden Konflikte zu erkennen und zu bearbeiten, damit das Ziel des Spiels erreicht werden kann. Es erfordert ein auf Selbstreflexion basierendes Verhalten, aufgrund dessen flexible Strategien entwickelt werden, um die Gegenseite (andere Kultur) besser zu verstehen. Die Spieler sind dabei auch emotional stark betroffen, da sie einerseits die Emotionen ausdrücken sollten, die ihren Vorstellungen oder Spielregeln entsprechen, andererseits sich auf unsicherem Boden (dazu gehören Angst, unerwartete Freude, Enttäuschung etc.) befinden, da sie den emotionalen Zustand der Gegenspieler nicht einschätzen können.

„Ziel des Simulationsspiels ist es, bei den Schülern/innen eine Sensibilisierung für die Eigenart von Kulturen, eine Reflexion über die Mechanismen der Vorurteilsbildung und erste Strategien zum Handeln im interkulturellen Feld zu erarbeiten“"(Ostendorf 2005, S. 8).

Das in PEIK zum Einsatz gekommene Simulationsspiel heißt „Poljane und Gorjane“ und wurde von der Verfasserin ausgearbeitet. ${ }^{40}$

Die Arbeit an dem Simulationsspiel wird in fünf Phasen eingeteilt:

1. Einführung der Teilnehmer in das Szenario und die Aufgabenstellung.

Unter dem Szenario wird folgender Kontext verstanden:

Poljane und Gorjane treffen sich, um eine Veranstaltung zu organisieren. Es geht dabei um eine Sportveranstaltung, etwas Ähnliches wie Olympische Spiele, aber im Kleinformat. Die beiden Gruppen wurden von ihren Kulturen entsandt, um die ersten Vorbereitungen zu treffen.

Der Lehrer stellt den Kontext vor. Im Folgenden erklärt er, dass alle Spielteilnehmer diese Situation wie ein Theaterstück nachspielen sollen.

2. Bildung von Kleingruppen, welche Kulturgemeinschaften repräsentieren, deren kulturelle Skripte ${ }^{41}$ durch Rollenkarten definiert sind.

Alle Spieler werden durch Zufallsverfahren ${ }^{42}$ in zwei Gruppen aufgeteilt, die zwei beteilig-

40 Das bekannteste Simulationsspiel in dem Gebiet des interkulturellen Trainings heißt BaFa'BaFa von Shirts (1977). Darin wurde die Begegnung zwischen den zwei Kulturen Alpha (männlich, kollektivistisch) und Beta (weiblich, individualistisch) simuliert. Unter anderem können folgende Simulationen erwähnt werden: Ecotonus von Hofner Saphiere (1995), „Fünf-Kulturen-Spiel“ und „Modis und Trados“ von Flechsig (1995, 2001), „Honkas und Eugenikern“ Hennig, Wahl \& Welker (2003), „Autobahnbau“ von Böttcher M. u.a. (2003)

${ }_{41}$ Kulturelle Skripte sind Rollenkarten, die die Aufgabenstellung und Spielregeln enthalten.

42 Zwischen den Spielern werden die Kärtchen verteilt: auf einigen Kärtchen werden Flussnamen 
te Kulturgemeinschaften „Poljane“ und „Gorjane“ präsentieren. Jede Gruppe bekommt ihre eigenen kulturellen Skripte. Die kulturellen Skripte sind in schriftlicher Form mitgeteilt, damit sie erlernt werden können. Hier ist das Skript-Beispiel für Poljane angeführt:

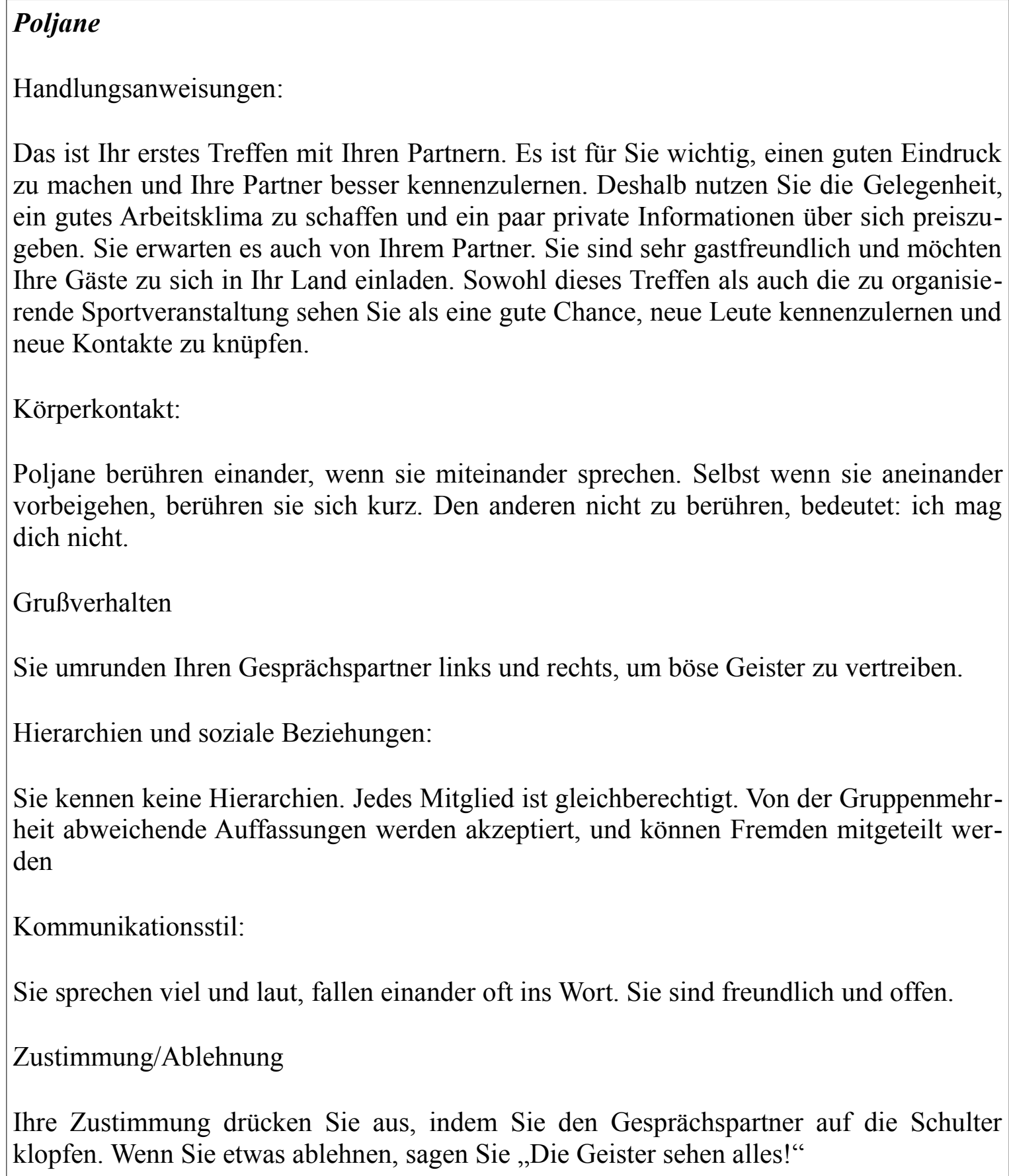

Das ist Ihr erstes Treffen mit Ihren Partnern. Es ist für Sie wichtig, einen guten Eindruck zu machen und Ihre Partner besser kennenzulernen. Deshalb nutzen Sie die Gelegenheit, ein gutes Arbeitsklima zu schaffen und ein paar private Informationen über sich preiszugeben. Sie erwarten es auch von Ihrem Partner. Sie sind sehr gastfreundlich und möchten Ihre Gäste zu sich in Ihr Land einladen. Sowohl dieses Treffen als auch die zu organisierende Sportveranstaltung sehen Sie als eine gute Chance, neue Leute kennenzulernen und neue Kontakte zu knüpfen.

Körperkontakt:

Poljane berühren einander, wenn sie miteinander sprechen. Selbst wenn sie aneinander vorbeigehen, berühren sie sich kurz. Den anderen nicht zu berühren, bedeutet: ich mag dich nicht.

Grußverhalten

Sie umrunden Ihren Gesprächspartner links und rechts, um böse Geister zu vertreiben.

Hierarchien und soziale Beziehungen:

Sie kennen keine Hierarchien. Jedes Mitglied ist gleichberechtigt. Von der Gruppenmehrheit abweichende Auffassungen werden akzeptiert, und können Fremden mitgeteilt werden

Kommunikationsstil:

Sie sprechen viel und laut, fallen einander oft ins Wort. Sie sind freundlich und offen.

Zustimmung/Ablehnung

Ihre Zustimmung drücken Sie aus, indem Sie den Gesprächspartner auf die Schulter klopfen. Wenn Sie etwas ablehnen, sagen Sie „Die Geister sehen alles!“

3. Kleingruppenarbeit: Erlernen der kulturellen Skripte und Entwicklung einer (Spiel-)Strategie.

Jede Gruppe bereitet sich auf das Treffen vor: Die Spieler lesen und eignen sich die kulturellen Skripte an, verteilen die Rollen, arbeiten an einer Verhaltensstrategie. In jedem gehören zu der Kulturgemeinschaft „Poljane“, die Besitzer der Städtenamen sind die „Gorjane“. 
Skript sind die kulturspezifischen Informationen angegeben wie Körperkontakt, Grußverhalten, soziale Beziehungen und Hierarchien, Kommunikationsstil. Es sind aber auch einige Informationen dabei, die sich auf die gemeinsame Aufgabe beziehen, z.B. in der Handlungsanweisung. Da steht, was die Vertreter einer Kulturgemeinschaft von dem Treffen mit einer anderen Gemeinschaft erwarten und was sie unter der Lösung dieser Ausgabe verstehen. Die Kleingruppenarbeit dauert 20 Minuten, die Gruppen bereiten sich in verschiedenen Räumen vor, damit die Informationen über die Skripte der anderen Gruppe vorenthalten bleiben.

4. Durchführung des Spieles - Interaktion der Kleingruppen gemäß Rollenkarten.

Die Simulation einer interkulturellen Kommunikation selbst dauert etwa 10 Minuten. Da die kulturellen Skripte der beiden Gruppen häufig gegensätzliche Anweisungen bezüglich des Verhaltens und der Lösungswege enthalten, ist zu erwarten, dass die Aufgabenlösung für alle Spieler nicht leicht fallen und zur Irritationen führen wird.

5. Auswertung und Diskussion des Verlaufs der Simulation und der dabei gewonnenen Lernerfahrungen.

Obwohl ein selbstreflektierendes Verhalten der Spieler im Laufe der gesamten Simulation erforderlich ist, bekommen sie nur in der fünften Phase die Möglichkeit, darüber zu reden. Ziele der Diskussion sind, die eigenen Gefühle und Erfahrungen auszuwerten und die kulturellen Skripte der anderen Gruppe zu entschlüsseln. Deshalb konzentriert sich die Diskussion auf drei Bereiche: eigene Kultur, fremde Kultur und Konfliktbearbeitung. Hier sind die Diskussionsfragen:

- Wie ernsthaft haben Sie die Rolle wahrgenommen?

- War es schwierig, die Rolle eines Poljanins/eines Gorjanins zu spielen?

- Wenn ja, welche Schwierigkeiten hatten Sie?

- Welche Gefühle löste das Verhalten Ihres Gesprächspartners bei Ihnen aus? Wie sind Sie mit diesen Emotionen umgegangen?

- Welche kulturellen Skripte von den gegenseitigen Gruppen haben Sie während des Spiels entschlüsselt?

- Welche Kommunikationsstörungen/Missverständnisse haben Sie wahrgenommen? Was waren die Gründe?

- Wie haben Sie versucht, mit den Missverständnissen umzugehen?

Zusammenfassend lässt sich Folgendes sagen: Die Fähigkeit zur Selbstreflexion spielt eine wichtige Rolle bei der Entwicklung der interkulturellen Identität. Deshalb wurde ein Teil 
der Aufgaben im PEIK der Arbeit an der kulturellen Selbstreflexion gewidmet, und zwar der Bewusstmachung der eigenen kulturellen Identität und der Sensibilisierung gegenüber den eigenen kulturellen Einstellungen. Eine Checkliste zur Erfassung der kulturellen Identität und Satzergänzungsübungen helfen den Teilnehmern, die Subkulturen anzusprechen, mit denen sie sich identifizieren, und sich deren zentrale Werte bewusst zu machen. Der Fragebogen zur Erhebung von Kulturkontrasterfahrungen macht die wichtigsten Kulturstandards bewusst, die das Verhalten der Teilnehmer steuern und die ein großes Potenzial an Differenzen gegenüber Vertretern anderer Kulturen besitzen. Im Laufe des Simulationsspieles „Poljane und Gorjane“ erleben die Teilnehmer eine künstlich geschaffene Situation einer interkulturellen Begegnung, worin sie lernen, ihr Verhalten bewusst zu steuern, ihre Gefühle zu kontrollieren, die Gründe für interkulturelle Missverständnisse zu verstehen. Die wichtigste Arbeitsmethode bezüglich der Entwicklung der Selbstreflexion ist die Diskussion, die von dem Lehrer eingeführt und moderiert wird. Die Anregungsfragen zu jeder Diskussion sind in PEIK vorhanden.

\subsubsection{Vorgefasste Meinungen und Stereotype. Arbeitsmethodik und Beispiele aus dem Programm}

\subsubsection{Ziele und Inhalte}

Nationale Stereotype sind anerkannte „Schädlinge“ der Kommunikation zwischen Vertretern unterschiedlicher Kulturen. Die Tatsache, dass wir die umgebende Welt durch die Brille unserer schematisierten Vorstellungen wahrnehmen, spiegelt sich folgendermaßen in unserer Ansicht über andere Völker wider: Bei Beginn einer interkulturellen Kommunikation sind wir schon mit bestimmten Vorurteilen gegenüber dem Gesprächspartner vorbelastet, die immer auch einen emotionalen Aspekt haben. Im ,ungünstigsten“ Fall droht die Kommunikation wegen der aufgrund dieses Vorurteils ausgewählten Verhaltensstrategie fehlzuschlagen. Im schlimmsten Fall sind Streit und Diskriminierung die Folgen der zu eifrigen Fixierung auf Vorurteile und Stereotype. Also, die logische Schlussfolgerung wäre: die Stereotype müssen weggeschafft werden. So sind in der Fachliteratur die Ausdrücke „Stereotype abbauen“ oder „Stereotype beseitigen“ oft anzutreffen. Sind aber diese Ziele realisierbar, sowohl in der Praxis des Fremdsprachenunterrichts als auch allgemein?

Die Neigung zur Stereotypenbildung ist auf das Bedürfnis unseres Gehirns zurückzuführen, die einzelnen Bilder der Wirklichkeit zusammenzufassen und in ein geordnetes System 
zu integrieren. Schemata zu bilden und mit ihrer Hilfe die äußeren Einflüsse zu verarbeiten, sind natürliche Vorgänge menschlicher mentaler Prozesse. Der Mensch ist auf diese Stereotypenbildung angewiesen, sonst könnte er die eigenen Eindrücke, seine einzelnen zerstreuten Bilder der Wirklichkeit, nicht bearbeiten. Diese soziale Orientierungsfunktion der Stereotype ist sehr wichtig für das Verstehen des anderen. Also: Schemata dienen dem Verstehen einer Sache, Stereotype haben darüber hinaus eine soziale Orientierungsfunktion, sie dienen dem Verständnis der Menschen. Man kann Stereotype nicht komplett abbauen, das widerspricht der Orientierungsfähigkeit des Gehirns. Man kann sie aber modifizieren. Dies versteht die Verfasserin als Aufgabe bei der Arbeit mit Stereotypen.

In der vorliegender Arbeit geht es nicht um Stereotype allgemein (sie können sich im Prinzip auf alle Gruppen beziehen, die anders sind, z.B. Behinderte, Straffällige, Bewohner einer anderen Stadt oder eines anderen Viertels), sondern um nationale Stereotype. Der gezielte Abbau nationaler Stereotype ist eine lobenswerte und nur bis zu einem bestimmten Grad erreichbare Zielvorstellung. Ein langer Aufenthalt in einem anderen Land (vielleicht auch in anderen Ländern) wäre eine der Grundvoraussetzungen dazu. Da PEIK aber auf den Hochschulunterricht zugeschnitten ist, ist die Möglichkeit, die vorhandenen Stereotype abzubauen, eher gering.

Die Aufgabe dieses Teil des PEIK ist, vorhandene nationale Stereotype zu thematisieren, sie und ihren Einfluss auf die interkulturelle Kommunikation bewusst zu machen und Strategien zu entwickeln, um im Weiteren mit nationalen Vorurteilen umgehen zu können.

Bei der Arbeit mit nationalen Stereotypen ist es wichtig zu wissen, dass sie auf allen drei Ebenen des Lernens bearbeitet werden sollten: Stereotypen existieren nicht nur auf kognitiven Ebene, sondern sind auch emotional gefärbt und dienen als Anleitung für das weitere Handeln. Ein Beispiel: Auf der emotionalen Ebene kann der Vertreter einer anderen Kultur Ablehnung, Misstrauen oder Mitleid hervorrufen; diese Emotionen begleiten die Meinung darüber, dass der Fremde faul, dreckig, ungebildet, kriminell ist; als Folge ist folgendes Verhalten zu beobachten: den Kontakt mit dem Fremden vermeiden, Informationen vorenthalten, vorsichtig/ nicht ehrlich sein. Die Arbeit mit den Stereotypen sollte dann auf allen drei Ebenen erfolgen.

Auf der kognitiven Ebene liegen die schematischen Bilder der fremden Kultur, die aufgrund der mangelnden oder fehlenden individuellen Erfahrung von anderen Vertretern der eigenen Kultur unkritisch übernommen wurden. Die erste Aufgabe ist, diese Bilder aufzudecken und sie den Teilnehmern bewusst zu machen. Die Feststellung der möglichen Entstehungsgeschichte der nationalen Stereotype wäre der nächste Schritt der Arbeit. Die Fest- 
stellung der Tatsache, dass sie zu verallgemeinerte Informationen über Sachverhalte darstellen, die dem Teilnehmer selbst unbekannt oder schlecht bekannt sind - wäre die logische Schlussfolgerung dieser Arbeit. Die Mitteilung möglichst umfangreicher Informationen und dadurch die Ausdifferenzierung des bestehenden Auslandsbildes sind hier zu empfehlen. „Die groben Verzerrungen der Fremdkultur durch die eingespeicherten Schemata lassen sich durch zusätzliche, differenzierende Informationen relativieren, ergänzen und erweitern“ (Bredella 1990, S. 566). Die neuen Informationen bringen einige Unstimmigkeiten in ein eher einheitliches Auslandsbild, dadurch entstehen Widersprüche, die zur Ausdifferenzierung dieses Bildes beitragen und letztendlich ausgewertet und in das neue Bild integriert werden sollten.

Da das Beibehalten des einheitlichen Auslandsbildes enorm wichtig ist für das Gehirn der Teilnehmer, sollten die neuen Informationen nicht vereinzelt mitgeteilt werden. Sie sollten in ein System eingebettet werden, das einen Überblick über die zeitlichen und kausalen Hintergründe dieser Informationen verschafft. Das ist durch die Vermittlung von geografischen, geschichtlichen und soziokulturellen Zusammenhängen möglich. Diese Funktion im Unterricht hat seit langem Landeskunde übernommen.

„Nur durch wechselseitige, sich gegenseitig ergänzende und oft sogar widersprechende Einbettung unterschiedlicher emotionaler, sozialer, sachlicher und moralischer Kategorien werden die kulturellen Stereotype in einem neutralen Wechselspiel der Module relativiert, modifiziert, interpretiert und bewertet und dabei in adäquate Verhaltensmuster umgesetzt ${ }^{\text {“* }}$ (Keller 2007).

Eine besondere Bedeutung wird der Vermittlung von Einstellungen und Werten zugemessen. Sie bilden ein Hintergrundwissen, ein „unsichtbares“, aber einflussreiches Netz, das eine entscheidende Rolle bei der Rekonstruktion eines Auslandsbildes spielt. Die Frage nach dem nationalen Charakter (Wie sind die Deutschen?) z.B. wird in diesem Zusammenhang in die Frage verwandelt: Welche Lebensgewohnheiten haben Jugendliche/ Dorfbewohner/ Friesen? Durch welche ökonomischen, technischen, kulturellen Gegebenheiten ist die Entstehung dieser Lebensgewohnheiten zu erklären?

Stereotype haben einen emotionalen Charakter. Aus diesem Grund ist es wichtig, auch diese Ebene bei der Arbeit in Betracht zu ziehen. Die Bilder über Völker können negativ oder positiv gefärbt sein. Im Regelfall sind Autostereotype (schematische Vorstellungen über das eigene Volk) positiver beladen, während Heterostereotype (schematische Vorstellungen über andere Völker) sowohl negative als auch positive Charakteristiken aufweisen. Die emotionale „Ladung“ von Stereotypen scheint der Hauptgrund für Diskriminierungen zu 
sein und auch dafür, dass vorhandene Schemata so schwer abzubauen sind. Als Aufgabe für den Fremdsprachenunterricht ist die Weckung positiver Assoziationen für fremde Völker zu nennen. Dieser Vorgang ist eng mit der Motivierung der Teilnehmer verbunden: durch die positive Einstellung zum Lerninhalt, in unserem Fall sind das die Fremdsprache und die Fremdkultur, kann die Motivation zum Unterricht erhöht werden.

Die bei der Entwicklung der interkulturellen Kompetenz bewährte Arbeitsmethode des Kulturvergleichs ist bei der Arbeit mit den Stereotypen aufgrund folgender Überlegung einzusetzen: Teilnehmer mit fehlender oder geringer Auslandserfahrung werden das in der eigenen Kultur erworbene Wissen über die fremde Kultur (das oft in Form von nationalen Stereotypen existiert) bei dem Vergleich der beiden Kulturen einsetzen. Deshalb ist es wichtig für den Fremdsprachenlehrer, darauf zu achten und die Teilnehmer darauf aufmerksam zu machen, dass die Schlussfolgerungen über das Fremde aufgrund von vorgetragenen Informationen und nicht aufgrund der vorgefassten Meinungen gemacht werden sollten. Die Rekonstruktion der fremden Realität aus der Sicht ihrer Vertreter durch authentische Texte muss im Fremdsprachenunterricht angestrebt werden.

Eine andere Arbeitsweise ist, nach Gemeinsamkeiten zu suchen. Um von der Kategorisierung nach der nationalen Zugehörigkeit abzulenken, müssen andere Kategorien geschaffen werden. So kann das Schaffen einer Überschneidungskategorie wie z.B. „Wir Jugendliche“ oder „Wir Polizisten“ in bestimmten Kontexten zur Auflösung der Kategorien „Wir Russen“ und „Die Deutschen“ führen.

\subsubsection{Didaktisch-methodische Umsetzung}

In PEIK werden folgende Aufgaben zum Thema „Stereotype“ eingesetzt: Fragebogen „Deutschland und die Deutschen“, Diskussionsrunde „Nationale Stereotype“, Unterrichtseinheit „Andere Länder in unseren Köpfen“, Unterrichtseinheit „Typisch Russisch?“.

Als Einführung dieses Teils des Programms wird ein Fragebogen „Deutschland und die Deutschen“ eingesetzt, der gezielt nach den stereotypen Bildern über die Nation, deren Sprache gelernt wird, fragt. Hier sind die Fragen:

- Nennen Sie Ihre Assoziationen zu den Wörtern „Die Deutschen“, „Deutschland“. Nennen Sie nicht weniger als 10 Begriffe zu jedem Wort. 
- Wurden Sie schon mit Vertretern der deutschen Nation konfrontiert? Wenn ja, beschreiben Sie Ihren Kontakt.

- Woher haben Sie Ihre Information über die Deutschen (Deutschland). Nennen Sie die Quellen.

- Welche deutschen Filme und Fernsehprogramme haben Sie gesehen, welche deutschen Bücher, Zeitungen, Zeitschriften haben Sie gelesen?

- Was halten Sie davon, wenn Ihr Mitbewohner ein Deutscher wäre?

In der ersten Frage werden die allgemeinen Stereotype zu den Wörtern „Deutsche“ und „Deutschland“ abgefragt. Bei der zweiten Frage wird festgestellt, ob die vorher angeführten Assoziationen meistens aus der eigenen Erfahrung gemacht wurden oder die in der eigenen Gesellschaft verbreiteten Bilder darstellen. Die Frage über die Art des interkulturellen Kontakts hat das Ziel, die Intensität des Kontakts zu prüfen. Wichtig für die weitere Diskussion ist die Frage drei: Das Nachdenken über die Quellen des Wissens über das fremde Land ist selbst ein Lernprozess, die Teilnehmer lernen zu verstehen, wie sehr ihre Assoziationen von dem Wissen abhängig sind, die sie aus den Medien bekommen haben, und welcher Teil ihrer Assoziationen ,aus der Luft ${ }^{\star 43}$ gegriffen wurde. Um einen weiteren Impuls in diese Richtung zu geben, wurde die Frage vier eingeführt. Die fünfte Frage ist auf die emotionale Einstellung der Teilnehmer zu den Deutschen ausgerichtet. Die Frage nach dem Wunsch, mit einem Deutschen zusammenzuwohnen, misst das Interesse der Teilnehmer an einem näheren Kontakt mit Vertretern der Zielkultur.

Die Ergebnisse des Fragebogens werden von den drei Kleingruppen ausgewertet, von denen jede sich mit zwei Fragen beschäftigt. Die Ergebnisse werden weiter im Plenum präsentiert.

Der nächste Schritt für die Thematisierung der nationalen Stereotype ist die Diskussion zu diesem Thema. Sie wird durch einen kleinen Vortrag eingeleitet, der die Definitionen der Wörter „Stereotyp“, „Vorurteil“" und „Nationalcharakter“ sowie allgemeine Informationen darüber enthält. Der Vortrag wird von einem der Teilnehmer mit der Hilfe des Lehrers vorbereitet. Am Ende dieses Vortrages präsentiert der Referent die „Völkertafel ${ }^{644}$ mit einer

\footnotetext{
43 Die Redewendung ,aus der Luft“ stammt aus dem Russischen. In diesem Zusammenhang bedeutet das, dass die Teilnehmer oft die Quelle vieler Informationen gar nicht nennen können. Das bedeutet, sie haben es aus der Umgebung, aus dem Alltag vermittelt bekommen. Im Grunde genommen kann gesagt werden, der Vermittler dieser Informationen ist die eigene Kultur selbst.

44 Völkertafel ist ein in der Steiermark im 18. Jahrhundert entstandenes Ölgemälde eines unbekannten
} 
selbst erarbeiteten Übersetzung.

Anschließend wird die vom Lehrer moderierte Diskussion durchgeführt. Zur Diskussion werden folgende Fragen gestellt:

1. Was fällt Ihnen ein, wenn Sie die Völkertafel aus dem 18. Jahrhundert sehen? Erkennen Sie die eigenen Vorurteile gegenüber anderen Völkern? Was denken Sie über die Vorurteile gegenüber den Russen?

2. Haben sich die nationalen Stereotype Ihrer Meinung nach im Laufe der Zeit geändert? Was können die Gründe für diese Veränderungen sein?

3. Was sind mögliche positive oder negative Folgen der nationalen Stereotype?

Die Aufgabe dieser Diskussionsrunde besteht darin, auf die theoretischen Grundlagen solcher Stereotype aufmerksam zu machen, z.B. dass Nationalbilder relativ konstant sind, auch dass einige geschichtliche Ereignisse darin ihre Spuren hinterlassen haben. Es wird damit gerechnet, dass die Konfrontation mit nationalen Vorurteilen gegenüber der eigenen Gruppe emotional sehr bedeutsam sind, besonders weil Vorurteile gegenüber Russen häufig emotional negativ ausfallen. Diese Einsichten vermitteln den Teilnehmern Anregungen, darüber nachzudenken, welches Bild der eigenen Kultur in anderen Kulturen existiert.

Die Arbeit an der Thematisierung der nationalen Stereotype wird in der Unterrichtseinheit „Andere Länder in unseren Köpfen “ fortgesetzt. ${ }^{45} \mathrm{Im}$ ersten Teil des Unterrichts erraten die Teilnehmer für verschiedene Nationen typische Gegenstände und Verhaltensweisen. Ein Arbeitsblatt mit kleinen Bildern dient als Vorlage. Auf einem weiteren Blatt ist die Karikatur eines Deutschen dargestellt. Der Arbeitsauftrag lautet: Was finden Sie typisch deutsch? Was hat der Künstler übertrieben? Im zweiten Teil der Unterrichtseinheit werden alle Teilnehmer in zwei Kleingruppen aufgeteilt. Die erste Kleingruppe stellt eine Collage zusammen, die ihre Vorstellungen über eine Nationalität darstellt. Die zweite Kleingruppe bereitet ein kleines Theaterstück zum Thema vor.

1. . Die erste Kleingruppe wählt selbst ein Thema für ihre Collage aus: es geht um ein konkretes Thema aus dem Alltag, z.B. „Familie“, „Arbeit“, „Freizeit“ in Bezug auf die Deutschen. Alle notwendigen Materialien wie Zeitungen, Zeitschriften, Stifte,

Malers, das heute als Quelle der nationalen Stereotypen betrachtet wird. Auf dem Gemälde sind einige europäische Völker abgebildet, denen bestimmte Eigenschaften zugeschrieben sind.

45 Diese Unterrichtseinheit basiert auf den Materialien, die von Ivica Lencova (1997), Deutschlehrerin aus der Slowakei, ausgearbeitet wurden. 
Schere sind vorhanden. Diese Collage bietet den Teilnehmern in einer spielerischen und handlungsorientierten Form die Möglichkeit, ihre Stereotype bewusst zu machen. Im weiteren Ablauf präsentiert sie der anderen Kleingruppe ihre Collage ohne Kommentare. Die Teilnehmer der zweiten Gruppe versuchen, die Bilder zu entziffern, was zu der Diskussion über den gemeinsamen Wissensstand führen sollte. Einige Bilder können vermutlich nicht entziffern werden, was als Anregung zur Diskussion über die Subjektivität der Wahrnehmung dienen kann.

2. . Die zweite Kleingruppe bereitet ein kleines Theaterstück vor, in dem Vertreter einiger Nationen dargestellt werden. In der Vorbereitungsphase werden sowohl das Szenario als auch die Rollen erarbeitet. Die Teilnehmer wählen das Thema auch selbst aus, es kann aber schon um ein Missverständnis oder einen Konflikt gehen. Im Stück sollten die nationalen Stereotype simuliert werden. Im Anschluss an die beiden Präsentationen findet eine Diskussion statt.

Das nächste Arbeitsthema betrifft Auto- und Heterostereotype von Russen. Es wird in der Unterrichtseinheit „Typisch Russisch?“ realisiert. Bei der Differenzierung der Stereotype spielt die Perspektive des Betrachters eine wichtige Rolle: Vorurteile gegenüber der eigenen Kultur bzw. über Vertreter der eigenen Gruppe werden Autostereotype genannt; Vorurteile, die eine ethnische Gruppe gegenüber der anderen hegt, werden Heterostereotype genannt. In der Regel sind Autostereotype positiver belegt als Hetereostereotype, sie sind auch oft differenzierter.

PEIK bereitet die Teilnehmer auf einen interkulturellen Kontakt vor, dabei ist die Konfrontation mit den Vorurteilen der Vertreter einer fremden Kultur gegenüber der eigenen Kultur unausweichlich. Da die Heterostereotype der Teilnehmer gegenüber anderen Nationen in den vorigen Unterrichtseinheiten schon thematisiert wurden, sind nun die Autostereotype der Russen an der Reihe: Mit welchen Vorurteilen kann man bei einem interkulturellen Kontakt rechnen? Die Autostereotype werden aber gezielt abgegrenzt, indem eine Subgruppe innerhalb einer Nation besprochen wird. Die typisch russischen Studierenden stehen im Vordergrund.

. Als erster Schritt wird den Teilnehmern angeboten, eine Liste der Eigenschaften zusammenzustellen, die sie als ,typisch russisch“ in Bezug auf die Studentenschaft definieren. Die Arbeit erfolgt in zwei Kleingruppen. In der zweiten Etappe wird die folgende Tabelle angeboten, bei der die angekreuzten Felder frei sind. Diese Tabelle stellt die Überarbeitung der Ergebnisse einer empirischen Untersuchung an der Universität Bielefeld zu Auto- und 
Heterostereotypen von deutschen und russischen Studierenden dar (vgl. Rösch 1998). Den Teilnehmern wird aufgegeben, die Übereinstimmungen zwischen der eigenen Aufzählung und der von deutschen Studierenden benannten Charakteristika von Russen herauszufinden. In der anschließenden Diskussion wird gefragt, wodurch die Unterschiede zu erklären sind. In der dritten Etappe werden die Teilnehmer aufgefordert, in den Kleingruppen die Gewichtung der einzelnen Eigenschaften einzuschätzen. Im Weiteren werden die Ergebnisse der Untersuchung auf einer Folie präsentiert:

\begin{tabular}{|c|c|c|c|c|}
\hline & (2) & (;) $\odot$ & $(-) \odot$ & $\begin{array}{l}\odot \odot \odot \\
\odot \ominus\end{array}$ \\
\hline offen & & $\mathrm{X}$ & & \\
\hline nationalistisch & $\mathrm{X}$ & & & \\
\hline fröhlich & & & $\mathrm{X}$ & \\
\hline gastfreundlich & & & & $\mathrm{X}$ \\
\hline selbstbewusst & $\mathrm{X}$ & & & \\
\hline stolz & & $\mathrm{X}$ & & \\
\hline bescheiden & & & $\mathrm{X}$ & \\
\hline trinkfreudig & & & & $\mathrm{X}$ \\
\hline feiern gern & & & $\mathrm{X}$ & \\
\hline nicht strebsam & $\mathrm{X}$ & & & \\
\hline herzlich & & $\mathrm{X}$ & & \\
\hline traditionsbewusst & & & $\mathrm{X}$ & \\
\hline unkompliziert & $\mathrm{X}$ & & & \\
\hline gesellig & & $\mathrm{X}$ & & \\
\hline familiär & & $\mathrm{X}$ & & \\
\hline & & & & \\
\hline
\end{tabular}

Im Anschluss findet eine Diskussion mit den folgenden Leitfragen statt:

1. Entsprachen Ihre Einschätzungen den Meinungen der deutschen Studenten? Was hat Sie am meisten überrascht?

2. Wodurch sind die Unterschiede zwischen den Auto- und Heterostereotypen zu erklären?

3. Wenn Sie schon einen interkulturellen Kontakt zu Deutschen hatten, haben Sie eine Bestätigung Ihrer Vorurteile gefunden? Oder war Ihr deutschsprachiger Partner ein „untypischer“ Deutscher?

4. In Laufe der interkulturellen Kommunikation merken Sie, dass Ihr fremdkultureller 
Partner sich auf Stereotype über die Russen bezieht. Welche Strategien können Sie dagegen entwickeln?

Zusammenfassend kann gesagt werden, dass kulturübergreifende Kenntnisse über nationale Stereotype und ihr Einfluss auf die interkulturelle Kommunikation in PEIK durch vier Unterrichteinheiten vermittelt wird: Fragebogen „Deutschland und die Deutschen“, Diskussionsrunde „Nationale Stereotype“, Unterrichtseinheit „Andere Länder in unseren Köpfen“ Unterrichtseinheit „Typisch Russisch?“. Dank der unterschiedlichen Aufgabenstellungen und Arbeitsmethoden (Fragebogen, Diskussion, Erstellung von Collagen, Vortrag) werden die Teilnehmer sowohl gegenüber den vorgefassten Meinungen über die anderen Nationen als auch über die eigene Nation sensibilisiert. Die russischen Autostereotype und Heterostereotype gegenüber den Deutschen werden thematisiert; in den Diskussionen werden sowohl die Gefahren, die vorgefasste Meinungen für die interkulturelle Kommunikation darstellen, als auch die Strategien für den Umgang mit Stereotypen besprochen. Dieser Teil des Programms hat das Ziel, die Fähigkeit zum Umgang mit vorgefassten Meinungen zu fördern.

\subsubsection{Landeskunde und Sprachübungen: Arbeitsmethodik und Beispiele aus dem Programm}

\subsubsection{Ziele und Inhalte}

Im letzten Teil des Programms werden die traditionellen Inhalte des Fremdsprachenunterrichts präsentiert, die am meisten dazu beitragen, die interkulturelle Kompetenz der Teilnehmer zu entwickeln. Unter den traditionellen Inhalten werden die Informationen verstanden, denen tradionell im Fremdsprachenunterricht ein Platz eingeräumt ist, und zwar Fremdsprache und Landeskunde des Ziellandes.

Im Gesprächen über die interkulturelle Kompetenz als neues Ziel des Fremdsprachenlernens ist nicht zu vergessen, dass eine interkulturelle Kommunikation ohne das Erlernen einer Fremdsprache gar nicht möglich wäre. Um mit Vertretern einer anderen Kultur überhaupt kommunizieren zu können, müssen die Teilnehmer in erster Linie einen für beide Seiten verständlichen Code beherrschen. Die gemeinsame Sprache ist ein Medium, ohne das auch bei gut entwickelten kulturübergreifenden Kenntnissen, interkultureller Sensibilität und interkulturellen Fähigkeiten eine Kommunikation zwischen Vertretern zweier unter- 
schiedlicher nationaler Kulturen scheitern wird. Das Beherrschen einer Fremdsprache ist ein wichtiger Teil der interkulturellen Kompetenz.

Jede Sprache ist, wie bekannt, kein neutrales Medium, sondern „Teil eines in Kultur eingebetteten Kommunikationssystems“ (Roche 2001, S. 4). Wie tief und in welchen Formen die Kultur ihren Ausdruck in der Sprache fand, wurde im Kapitel 4.1.1. „Kulturspezifische Kenntnisse“ schon diskutiert. Die Vermittlung kulturspezifischer Kenntnisse war immer ein Teil des Unterrichts, sie wurden aber in der Praxis als landeskundliche Informationen „,verkauft“ und oft nur am Rande des Unterrichts. Unter dem interkulturellen Ansatz gewinnen sie aber einen besonderen Stellenwert. Aus dem „Kellerkind““46 Landeskunde entwickeln sich kulturelle Informationen, die auch durch die Sprache vermittelt werden können und die die Grenze „fact \& figures“ weit überschreiten. „Die kulturellen Lehransätze können daher wesentlich effizienter und effektiver sein als traditionelle Unterrichtsverfahren, die kulturelle Faktoren nur marginal behandeln“ (Roche 2001, S. 4).

Neben der Arbeit an Selbstreflexion, kulturellen Missverständnissen und nationalen Stereotypen wurden in PEIK auch neue Wege gesucht, durch die Sprache und durch die traditionelle Landeskunde interkulturelles Wissen zu vermitteln. Im vierten Teil des PEIK sind Übungen enthalten, die nicht nur rein kommunikative Fähigkeiten der Teilnehmer entwickeln, sondern auch ihre interkulturelle Sensibilität und andere interkulturelle Teilfähigkeiten wie Stereotypenkompetenz, Fähigkeit zur Selbstreflexion oder Fähigkeit zur konstruktiven Auseinandersetzung mit dem Eigenen und dem Fremden.

\subsubsection{Didaktisch-methodische Umsetzung}

Der vierte Teil des PEIK schließt folgende Arbeitsinhalte ein: Vortrag zum Thema „Einheit des Verschiedenen“, Spielstunde „Konferenz der Tiere“, Sprachübung „Spaghetti für zwei“, Spielstunde „Dill Dominoso“, Unterrichtseinheit „Kulturelle Regeln und Tabus in Minidialogen“, Unterrichtsstunde „Körpersprache“, Vergleichsanalyse eines Märchens „Der Wolf und die sieben Geißlein“, Vergleichsanalyse „Phraseologische Redewendungen “ und Diskussion „Mein Deutschland“.

Vortrag zum Thema „Einheit des Verschiedenen“. Er hat als Ziel, die Vielfalt der in Deutschland lebenden Subkulturen zu präsentieren. Die Informationen über die unterschiedlichen Volksgruppen, Dialekte und die nationalen Kulturen der in Deutschland le-

46 Der Begriff wurde von Volkmann (2002, S. 14) gebraucht. 
benden Ausländer sollen zusätzlich zu den im Lehrbuch schon enthaltenen landeskundlichen Informationen dazu verhelfen, das einheitliche Auslandsbild zu differenzieren. Ein kurzer Geschichtsüberblick stellt einen systematischen Zusammenhang zur modernen Realität dar, wo innerhalb der deutschen Gesellschaft regionale (mit Bundesländern teilweise korrespondierende) Bevölkerungsgruppen existieren. Dies löst die monokulturellen Vorstellungen über Deutschland und „die Deutschen“ auf. Das Bild wird durch einen statistischen Überblick über die Einwanderung und die Verteilung der einzelnen Volksgruppen ergänzt. Der Vortrag wird von zwei Teilnehmern auf Grundlage von Internetmaterialien vorbereitet.

Unterrichtssequenz „Spaghetti für zwei“ stellt eine von Sonja Leban, Breda Premzl und Elizabeta Puklavec ausgearbeitete Unterrichtseinheit zum Thema Missverständnisse dar. Die Autorinnen unternehmen den Versuch, aufgrund der Bearbeitung eines Textes interkulturelle Gedanken zu vermitteln. Ihr Ziel definieren sie wie folgt: „Vorrangiges Anliegen bei dieser Unterrichtssequenz ist, Textverständnis zu erzielen und die Thematik der Geschichte ,Vorurteile“ bewusst zu machen“ (Leban, Premzl und Puklavec 1998, S.1) Als Grundlage für diese Spracharbeit dient ein Text, in dem die Begegnung eines Jungen mit dem Namen Heinz mit einem afrikanischen Jungen (Marcel) in einem Selbstbedienungsrestaurant beschrieben ist. Der Text ist von Heinz' Gesichtspunkt erzählt. Im Grunde handelt es sich hier um ein Missverständnis, das aufgrund nationaler Vorurteilen entstanden ist.

Die Unterrichtssequenz nimmt zwei Unterrichtseinheiten ein. Die Arbeit im ersten Teil schließt eine Vorentlastungsphase ${ }^{47}$, eine Lesephase und Nachübungen ein. Der Text wird zunächst nicht bis zum Ende gelesen, damit die Teilnehmer ihre Hypothesen über den weiteren Ablauf der Geschichte samt Ende der ersten Unterrichtseinheit in Form eines kurzen mündlich vorgetragenen Textes bilden können. Im der zweiten Unterrichtseinheit verlagert sich der Schwerpunkt der Arbeit von der sprachlichen Seite auf den interkulturellen Aspekt. Nachdem die früher vorgestellten Hypothesen auf einem Plakat gesammelt und dabei wiederholt wurden, lesen die Teilnehmer das Ende der Geschichte, die dieses Missverständnis aufklärt, und vergleichen es mit ihren Hypothesen. Danach folgt die Gruppenarbeit mit den unterschiedlichen Aufgabenstellungen und die Diskussion. Abschließend schreiben die Teilnehmer diese Geschichte vom Gesichtspunkt des afrikanischen Jungen im Form eines Briefes, den Marcel seinen Verwandten nach Afrika schickt.

47 Vorentlastung wird im Fremdsprachendidaktik eine Unterrichtsphase genannt, wo ein neues Thema unter Nutzung des Vorwissens eingeführt wird. 
Diese Unterrichtssequenz stellt ein gutes Beispiel dafür dar, wie durch den inhaltlich aufschlussreichen Text, durch passend ausgearbeitete Aufgaben und durch den Aufbau des Unterrichts, der nach interkulturellen Gedanken konzipiert ist, die interkulturelle Kompetenz auch durch die einfache Spracharbeit vermittelt werden kann.

„Dill Dominoso“ ist ein Sprachdomino zum Erlernen einfacher Redemittel in verschiedenen Sprachen, das im Rahmen des Projektes LIFE ${ }^{48}$ von Hölscher (1997) entwickelt wurde. Die Unterrichtseinheit Spielstunde „Dill Dominoso“ ist in Form eines interkulturellen Sprachspiels konzipiert. Spiele sind anerkannte Lernmethoden, die auf dem natürlichen Spieltrieb der Menschen gründen. Sie sprechen nicht die kognitiven Fähigkeiten der Menschen an, sondern appellieren auch an andere Sinneskanäle, die Spielteilnehmer sind motiviert, was dauerhafte Verhaltens- und Einstellungsänderungen erzeugt.

„Das interkulturelle Spiel bietet nun die Möglichkeit, Alltagskulturerfahrungen anderer Kulturen zu machen. Hieraus kann sich ein viel tieferes Verständnis der eigenen, wie auch den fremden Positionen entwickeln, als dies durch kulturelle Begegnungen alleine möglich wäre. Durch das spielerische Einnehmen fremder Rollen wird ein Perspektivenwechsel vollzogen, der einen emotionalen Zugang zur Welt des anderen öffnet. Diese Erfahrungen bieten dann die Möglichkeit, unser eingefahrenes Verständnis der Welt weiter zu öffnen und eine neue Basis kultureller Wahrnehmung anzulegen. Der große Vorteil des Spiels besteht darin, dass diese Erfahrungen gleichsam gefahrlos ausprobiert werden können“ (Hölscher 1997, S. 2).

Aus mehreren Kartensätzen, die in diesem Spiel zur Verfügung stehen, wurden für PEIK zwei ausgewählt: „deutsch-russisch“ und „deutsch-deutsch“. Die vorhandenen Redewendungen werden zuerst im Unterricht eingeübt, die Teilnehmer bekommen eine Übersicht vor dem Spiel. Gespielt wird in zwei Untergruppen, die später ihre Kartensätze austauschen. Die Spielregeln gleichen denen von Domino, wo jeder Teilnehmer versucht, aus den vorhandenen Karten eine passende zu den schon auf dem Tisch aufgebauten „Kartenschlangen“ zu finden.

Ein weiteres interkulturelles Spiel, das auch auf den Gebrauch der Fremdsprache Deutsch angewiesen ist und zum Teil in PEIK aufgenommen wurde, ist „Konferenz der Tiere“ ${ }^{49}$.

48 LIFE (Interkulturelle Erfahrungen durch handelndes Lernen) ist ein Kooperationsprojekt für interkulturelles Lernen zwischen BMW Group und dem Staatsinstitut für Schulpädagogik und Bildungsforschung.

49 Das Spiel wurde aus dem LIFE entnommen. Seine Verfasser sind Behnken, Brückner, Grummes-Unruh, Ilmberger, Karaduman, Stürmer, Worframm. 
Es geht dabei um eine sprachliche Variation des Kartenspiels „Quartett“. Das Spielmaterial besteht

20 Spielkarten, auf denen fünf Tiere abgebildet sind. Auf den einzelnen Karten stehen zusätzlich Hauptwörter, die diese Tiere in vier Sprachen benennen, und Interjektionen, Laute, die die Tiere in dieser Sprache produzieren. Jeder Teilnehmer bekommt eine Karte, versucht das Tier, das dort abgebildet ist, nachzuahmen und seine Artgenossen zu finden. Das Hauptziel dieses Spiels ist, ein gesamtes Tierquartett zu bilden.

Dieses Spiel ist für das interkulturelle Lernen insofern interessant, als nicht nur lustbetontes Erlernen der Tiernamen in den verschiedenen Sprachen erzielt wird, sondern weil auch in spielerischer Form erkennbar wird, dass in verschiedenen Sprachen die Wiedergabe von Tierlauten unterschiedlich erfolgt. Eine weitere Anwendung des Spiels ist das Einüben der Strukturen zum Äußern von Wünschen und des Akkusativs. ${ }^{50}$

Bei der Vergleichsanalyse des Märchens „Der Wolf und die sieben Geißlein“ wurde eine Textarbeit mit kontrastiven Methoden kombiniert. Märchen stellen Gedankengut einer Nation dar und erlauben dem Lernenden einen Einblick in die Kultur des Zielsprachlandes. Märchen sprechen immer Emotionen an, deshalb lässt die Arbeit daran fast niemanden gleichgültig. Ein anderer Grund, im Fremdsprachenunterricht Märchen als Lerntexte zu nehmen, liegt an ihrer leichten und verständlichen Sprache. Das Märchen „Der Wolf und die sieben Geißlein“" spricht die Teilnehmer auch deshalb an, weil sie es aus der frühen Kindheit kennen. Das Märchen existiert sowohl in der russischen als auch in der deutschen Sprache. Es ist aber den Teilnehmern nicht immer bewusst, dass die Inhalte in unterschiedlichen Sprachen auch unterschiedlich sein können. Die Erkenntnis, dass scheinbar Bekannte in der fremden Kultur anders aussieht und andere Akzente bekommt, ist das interkulturelle Ziel dieser Unterrichtseinheit.

Für die Vergleichsanalyse wurde die deutsche Version des Märchens ausgewählt, die von den Brüdern Grimm geschrieben wurde. Als russische Version wurde der von der Filmgesellschaft „Sojusmultfilm“ 1957 produzierte Zeichentrickfilm mit dem gleichen Titel ausgewählt. Im Titel dieses Films ist angezeigt, dass er nach dem russischen Volksmärchen gezeichnet ist.

Als Einführung ins Thema erstellen die Teilnehmer einen Wortigel ${ }^{51}$ mit Assoziationen zum

50 Es kann als Kartenspiel benutzt werden. Die auf den Karten vorgegeben Sprachstrukturen wie ,Ich brauche....“, „Gib mir bitte....“ etc. ermöglichen den Teilnehmern den Ausdruck der Wünsche einzuüben. Die Aufgabe dabei besteht darin wie in dem ersten Spiel das gesamte Tierquartett zu bilden.

51 Wortigel ist eine Art Assoziogramm. Der umkreiste Ausgangsbegriff steht in der Mitte, von diesem Kreis gehen „igelförmig“ zahlreiche Striche, zu denen Begriffe geschrieben werden, die mit dem 
Thema „Märchen“. In der ersten Phase wird die Textarbeit an der deutschen Variante des Märchens durchgeführt. Die Teilnehmer bekommen zwölf ungeordnete Bilder, die sie in der richtigen Reihenfolge legen müssen. Danach bilden die Teilnehmer zwei Untergruppen, von denen jede einige Teile von dem zerschnittenen Text bekommt. Die Teilnehmer müssen die Teile lesen, verstehen und den Bildern zuordnen. Am Ende dieser Phase wird die deutsche Version des Märchens komplett gelesen. In der zweiten Phase des Unterrichts wird der aus der Kindheit bekannte Zeichentrickfilm angesehen. In der letzten, für das interkulturelle Lernen entscheidenden Phase werden die deutsche und die russische Version des Märchens in tabellarischer Form verglichen.

Arbeit an festen Redewendungen und Idiomen wird in der Unterrichtseinheit Wettbewerb „Phraseologische Redewendungen“ durchgeführt. Idiome bieten ein breites Potenzial an Möglichkeiten, ins Fettnäpfchen zu treten: sie brauchen nur wörtlich übersetzt zu werden. Meistens bestehen sie aus einfachen Wörtern, die in der Kombination für einen Nichtmuttersprachler oft keinen Sinn ergeben. Für den Muttersprachler sind sie selbstverständlich, er bemerkt die Problematik des Verstehens dieser Redewendungen oft nicht. Es ist ihm auch nicht bewusst, dass bei einer misslungenen Kommunikation nicht die einzelnen Wörter erklärt werden sollten, sondern der Sinn der ganzen Redewendung.

Aus diesem Grund wird bei der Bearbeitung dieses Themas ein großer Teil der Arbeit an russischen Redewendungen gewidmet. Die Aufgabe dieser Selbstreflexionsarbeit ist es, besonders gebräuchliche, in der russischen Sprache idiomatische Redewendungen zu thematisieren und die Tatsache bewusst zu machen, dass die wörtliche Gestaltung solcher Ausdrücke nicht mit ihrer Bedeutung gleichgesetzt werden kann. Eine weitere Aufgabe ist es, russische und deutsche Redewendungen zu vergleichen. Durch diese Arbeit wird den Teilnehmern bewusst, dass die Bedeutung eines und desselben Sachverhaltes in verschiedenen Sprachen durch unterschiedliche Redewendungen ausgedrückt werden kann.

Der Unterricht verläuft wie folgt: Die Teilnehmer bilden zwei Mannschaften, die im wechselnden Verfahren eine Antwortchance bekommen. Für die richtige Antwort bekommen sie Punkte, bei einer falschen Antwort geht die Antwortchance an die andere Mannschaft. Am Anfang werden die Bilder präsentiert, die die russischen Redewendungen präsentieren. Die Mannschaften erraten, welches Idiom damit gemeint sein könnte. Danach müssen sie die Bedeutung der Redewendung erklären. In der zweiten Hälfte versuchen die Teilnehmer aus der angebotenen Liste mit deutschen idiomatischen Redewendungen eine passende zu den

Ausgangswort assoziiert werden. 
russischen zu finden.

Nonverbale Kommunikation ist alles andere als eindeutig, deshalb darf dieser Aspekt der Kommunikation bei der Arbeit an der interkulturellen Kompetenz der Teilnehmer nicht vergessen werden. Mimik, Gestik, Körpersprache etc. ist ein wortloser Teil der Kommunikation. Die Aufmerksamkeit der Teilnehmer wird auf diese Problematik durch die Unterrichtseinheit „Körpersprache“ gelenkt.

Das Ziel dieser Unterrichtsstunde besteht darin, den Teilnehmern bewusst zu machen, dass die eigenen Gesten von Vertretern der fremden Kultur anders als in der Heimatkultur interpretiert werden können. Es ist unmöglich, die eigene Körpersprache ,auszuschalten“, aber es ist empfehlenswert, bei einer interkulturellen Kommunikation auf sie zu achten, genauso wie auf die Reaktion des Gesprächspartners auf eigene Gesten und Körperhaltungen. Das Ziel ist nicht, den Teilnehmern die Bedeutung aller Gesten des deutschsprachigen Raumes in dieser Unterrichtseinheit beizubringen. Es ist wichtiger, sie für diesen Aspekt der Kommunikation zu sensibilisieren, damit sie das Verhalten eines fremdkulturellen Partners nicht voreilig interpretieren.

Als Einführung bereiten zwei Teilnehmer ein kurzes Referat über die nonverbale Kommunikation vor. Der Inhalt des Referats muss die folgenden Punkte beinhalten:

1. Was gehört zur Körpersprache?

2. Beispiele von unterschiedlichen Interpretationen der gleichen Gesten in unterschiedlichen Kulturen

3. Beispiele von aufgrund fehlerhafter Interpretation misslungener interkultureller Kommunikation.

Im weiteren Ablauf des Unterrichts bilden die Teilnehmer vier Kleingruppen, von denen jede die Aufgabe bekommt, einen kleinen Dialog nachzuspielen. Im Aufgabenblatt ist auch eine Geste aus dem deutschsprachigen Raum mit ihrer Interpretation angegeben. Nach der Vorbereitungszeit werden die Dialoge nachgespielt, die Zuschauer erraten die Bedeutung der Gesten. Zum Abschluss wird eine kleine Feedbackrunde mit folgender Leitfrage durchgeführt: Wie habe ich mich bei der Beobachtung der fremden Gestik gefühlt?

Unterrichtseinheit „Kulturelle Regeln und Tabus in Minidialogen“. Sie befasst sich mit Situationen des Alltagslebens, die ein Potenzial an aus kultureller Sicht der Muttersprachler falschen Reaktionen haben. Dabei wird die Aufmerksamkeit der Teilnehmer auf das akzeptable Sprachverhalten fokussiert. In dieser Unterrichtseinheit werden Texte präsentiert, in denen Aspekte akzeptierten oder nicht akzeptierten Sprachverhaltens aufgezeigt und pro- 
blematisiert sind. „Die Ausstattung mit Fähigkeiten zur erfolgreichen Kommunikation in interkulturellen Kontexten bedarf der Ergänzung des auf Kulturwissen bezogenen Inhaltsbereichs um solche Bestände, die auf Kooperation und Akzeptanz der Lernenden bei kommunikativen Anlässen auf Englisch ausgerichtet sind“, so Gehring (2002, S.92) bezüglich des ,Englisch als Fremdsprachenunterrichts‘. Das Ziel dieser Übung ist es, die Teilnehmer für das dem Etikett entsprechenden Sprachverhalten zu sensibilisieren. Die Übung ist in der Form von kleinen Situationen oder Minidialogen gefasst, wobei für die Ausgangssituation mehrere Alternativen angegeben sind Hier ist ein Beispiel dazu:

Sie sind zu Gast in einer deutschen Familie. Alle Gäste werden zu Tisch gebeten, Sie müssen aber zur Toilette. Was machen und was sagen Sie:

- „Entschuldigung, wo ist hier die Toilette?“

- Wo kann ich meine Hände waschen?“

- „Darf ich Ihr Badezimmer benutzen?“

- Sie sagen nichts und versuchen selbst die Toilette zu finden

Zum interkulturellen Lernen gehört es nicht nur, die Unterschiede zwischen den zwei Kulturen zu zeigen, sondern auch die Gemeinsamkeiten. Im Laufe des Programms haben die Teilnehmer unterschiedliche Erscheinungen aus der fremden Kultur kennengelernt, sie haben sich auch die eigene kulturelle Prägung bewusst gemacht, sie wurden für dieses „Verschiedensein“" sensibilisiert. Um den Perspektivenwechsel zu ermöglichen, der wichtig für das interkulturelle Lernen ist, muss noch ein Aspekt des Lernens beachtet werden, und zwar die Gemeinsamkeiten. Dieses Ziel wird bei der Diskussion „Mein Deutschland“ verfolgt. Die Gemeinsamkeiten zwischen der eigenen Kultur und der Kultur der Fremdsprache zu erkennen, wäre eine positive Einstellung zur Zielkultur wünschenswert, das bedeutete auch, dass die Motivation zum Erlernen der Fremdsprache wächst. Da davon ausgegangen wurde, dass die Teilnehmer keine oder wenig persönliche Erfahrung bezüglich des Ziellandes haben, wurde zwei Geschichte, ein deutsches und ein russisches für das oben genannte Ziel ausgewählt. Am Anfang findet ein Brainstorming statt, bei dem die Teilnehmer bekannte Persönlichkeiten oder Ereignisse nennen, die die beiden Länder in der Vergangenheit zusammenbrachten. Dann werden den Teilnehmern zwei Gedichte angeboten: zum einen der Text der deutschen Nationalhymne, also das Lied der Deutschen, und zum anderen das Gedicht der russischen Dichterin Marina Zwetaeva „Mein Deutschland“. Das Deutschlandlied wurde von August Heinrich Hoffmann von Fallersleben im Jahre 1841 ge- 
schrieben und im Jahre 1922 zur Nationalhymne erklärt. Obwohl der Text von 1922 heute nicht mehr gesungen wird, eignet er sich m.E. für den Fremdsprachenunterricht (vgl. auch Pjatschina 2002, S. 157). Die russische Dichterin Marina Zwetaeva, die sich schon seit ihrer Kindheit mit Deutschland verbunden fühlte, von der deutschen Literatur begeistert war und einige Monate in Berlin in der Verbannung verbrachte, schrieb 1914 das Gedicht „Mein Deutschland“. (Siehe Anhang 2)

In dem russischen Text berichtet die Dichterin über ihre Liebe zur deutschen Kultur und nennt viele eigene Assoziationen dazu. Dabei kommen aber auch die doppelseitigen Gefühle der Dichterin zum Ausdruck, weil viele Andeutungen auf die militärische Ausrichtung der damaligen deutschen Politik zu finden sind. Die Besprechung dieser Gedichte und der anschließende Austausch über die gemeinsame Vergangenheit unserer Länder, die sowohl positive als auch negative Seiten, enthält, schließen diesen Teil des Programms ab.

Zum Abschluss des Programms zur Entwicklung der Interkulturellen Kompetenz werden den Teilnehmern die Münchner Thesen zum interkulturellen Lernen angeboten. Diese Thesen verstehen sich als Zusammenfassung für die Programminhalte, die im Laufe des Experiments erlernt wurden. Die Thesen wurden von einem Arbeitskreis im Staatsinstitut für Schulpädagogik und Bildungsforschung in München als Leitsätze zum Profil einer interkulturellen Schule entwickelt. Im PEIK werden sechs aus neun vorhandenen Thesen eingesetzt, da das Problem der Interkulturalität hier innerhalb des Fremdsprachenunterrichts behandelt wird und nicht die Spezifika einer multikulturellen deutschen Schule hat. Hier sind die Thesen:

Menschen unterschiedlicher Herkunft, Sprache und Kultur leben in der heutigen Welt nahe beieinander.

Interkulturelles Lernen ist deshalb eine selbstverständliche Reaktion auf die veränderte Wirklichkeit.

Wir sind offen für Begegnungen mit Fremden: sie erweitern und ergänzen unsere Erfahrungen.

Nicht alles verstehen wir. Wir respektieren aber auch das, was uns fremd bleibt.

Wir achten darauf, dass der Fremde selbst sprechen kann, und bringen dabei unsere eigene Perspektive in den Dialog ein.

Die anerkannte Verschiedenheit führt uns zu einer kooperativen und solidarischen Haltung und bestimmt ganz selbstverständlich unsere Arbeits- und Lernformen.

Quelle: Arbeitskreis Staatsinstitut für Schulpädagogik und Bildungsforschung 1997. 
Die Arbeit an den Thesen wird in Form eines Stationsunterrichts durchgeführt. Jede These, die auf einem einzelnen Faltblatt gedruckt ist, wird im Unterrichtsraum aufgehängt. Die Teilnehmer haben etwa 20 Minuten Zeit, alle Thesen durchzulesen und mit den anderen zu besprechen. Dabei haben sie die Wahl, wie lange sie an jeder These arbeiten und welche Sprache sie dabei verwenden. Danach findet eine gemeinsame Diskussion darüber statt, welche Themen bei den Gruppengesprächen entstanden sind. Anschließend wird die Frage gestellt, was die Teilnehmer im Laufe des Programms gelernt haben und was sie für ihr weiteres Leben davon mitnehmen werden.

Zusammenfassend zum Kapitel 4 lässt sich sagen, dass sich das hier vorgestellte Programm zur Entwicklung der interkulturellen Kompetenz als ein Versuch versteht, den Unterricht Deutsch als Fremdsprache neu zu gestalten, insbesondere im Hinblick auf das interkulturelle Lernen. Dabei werden die Arbeitsinhalte und die Arbeitsmethoden des interkulturellen Trainings eingesetzt und die traditionellen Inhalte des Deutschunterrichts dem interkulturellen Kontext angepasst.

\section{Das Experiment zur Umsetzung des Puzzle-Modells im Fremdsprachenunterricht}

\subsection{Zielstellung des Experiments}

Das im Kapitel 5 vorgestellte Programm richtet sich an Teilnehmer des Deutschunterrichts, die die deutsche Sprache in einer weiterführenden Bildungseinrichtung erlernen. PEIK ist für Teilnehmer mit Vorkenntnissen einer Fremdsprache bestimmt, wird aber unter teilweiser Einbeziehung der Muttersprache durchgeführt.

Dieses Programm wurde im Rahmen eines Experiments in einer russischen Hochschule ausprobiert. Es fand 2004-2005 in dem Ost-Sibirischen Institut des Ministeriums des Inneren in der Stadt Irkutsk statt. PEIK wurde im Unterricht „Deutsch als-Fremdsprache“ im Laufe eines Wintersemesters eingesetzt. Am experimentellen Unterricht nahmen zwei Lerngruppen teil, die an der juristischen Fakultät der Hochschule in den Fachrichtungen „Recht“ und „Justiz“ eingeschrieben waren.

Interkulturelles Lernen in einer Hochschule in Russland, die sich nicht mit dem Sprachenlernen, sondern mit dem Erlernen eines Berufes befasst, hat in den meisten Fällen keine 
Tradition. Keine Ausnahme bildet die für das Experiment ausgewählte Hochschule. Aus folgenden Gründen wären aber interkulturelle Informationen für die Studierenden von Vorteil:

- Die Studierenden sind unterschiedlicher nationaler Herkunft, neben Russen sind die Ausbildungsteilnehmer Burjaten, Jakuten, Kasachen und Vertreter anderer Nationen sowohl aus dem asiatischen als auch aus dem europäischen Teil des Landes.

- Die weitere berufliche Tätigkeit der Studierenden wird der öffentliche Dienst sein. Mit Sicherheit kann behauptet werden, dass sie in der Zukunft sowohl mit ausländischen Bürgern als auch mit russischen Bürgern unterschiedlicher nationaler Herkunft konfrontiert werden.

Aus diesen Gründen sollte die Entwicklung der interkulturellen Kompetenz ein Teil der beruflichen Ausbildung sein. Dieses Ziel verfolgt der Einsatz von PEIK im Unterricht „Deutsch als Fremdsprache“.

Es wird vermutet, dass die interkulturellen Ziele von PEIK gut im Fremdsprachenunterricht eingegliedert werden können. Durch interkulturelle Inhalte soll das Entwicklungsniveau der interkulturellen Kompetenz steigen. Um dies zu überprüfen, werden die ersten Messungen gemacht, und zwar nicht nur in den zwei experimentellen Gruppen, sondern auch in zwei Kontrollgruppen vor und nach dem Experiment. Die Entwicklung der Kompetenz wurde mit drei Variablen gemessen: interkulturelle Kenntnisse, interkulturelle Sensibilität und interkulturelle Fähigkeiten. Diese abhängigen Variablen entsprechen den Bestandteilen des Puzzle-Modells.

Die Hauptarbeitshypothese des Experiments kann wie folgt formuliert werden: PEIK kann im Fremdsprachunterricht, der durch interkulturelle Ziele und Inhalte ergänzt wird, angewandt werden.

Es wird außerdem vermutet, dass das Niveau interkultureller Kompetenz der Lernenden steigt, wenn im Unterricht gezielt daran gearbeitet wird, den Lernenden kulturspezifische und kulturübergreifende Kenntnisse beizubringen, dabei ihr Bewusstsein für die Besonderheiten einer interkulturellen Situation erweckt wird und sie für diese Besonderheiten sensibilisiert sowie interkulturell bedeutsame Fähigkeiten entwickelt werden.

Die Verfasserin geht aber davon aus, dass die drei oben genannten Variablen sich nach dem Einsatz des PEIK nicht gleichmäßig entwickeln werden. Wie schon bei der Diskussion des Modells beschrieben (vgl. Kapitel 4), gibt es unterschiedliche Gründe für solch ungleiche Entwicklung. Folgende Punkte sollten im Laufe des Experiments bestätigt oder widerlegt werden: 
1. Es wird erwartet, dass die Variable „Kenntnisse/Bewusstsein“ die stärksten positiven Veränderungen erfährt. Die Aneignung von Kenntnissen ist ein Prozess, dessen Erfolg sich relativ gut bewerten lässt. Da es im Deutschunterricht, der im Laufe des Experiments durch Inhalte des PEIK ergänzt wurde, immer darum ging, sowohl kulturspezifische als auch kulturübergreifende Kenntnisse zu vermitteln, können die Lernenden ihre Erfolge selbst gut wahrnehmen. Vermutlich werden sie diese auch in ihrem Posttest registrieren.

2. Bei den Variablen „Sensibilität“ und „Fähigkeiten“ sind Veränderungen zu erwarten, aber sie werden vermutlich nicht groß sein. Dies hat seinen Grund darin, dass die Entwicklung von Emotionen ein lebenslanger Prozess ist. Die Zeitspanne eines Semesters dürfte zu gering für bedeutsame Veränderungen sein. Die Entwicklung sowohl der Sensibilität als auch der Fähigkeiten benötigt außerdem eine aktive Teilnahme an praktischen Handlungen, in unserem Fall an interkultureller Kommunikation. Im Unterricht „Deutsch als Fremdsprache“ besteht lediglich die Möglichkeit, künstlich geschaffene Situationen interkultureller Begegnung mit Vertretern der Zielkultur zu realisieren. Die Zielkultur wird nur bedingt erlebt, nämlich durch die Lehrmaterialien und die Persönlichkeit des Lehrers.

$\mathrm{Zu}$ den Zielen des Experiments gehört es auch, die Auswirkungen von Auslandserfahrungen und interkulturellen Erfahrungen, des Geschlechts und der Gruppenzugehörigkeit auf die Entwicklung der interkulturellen Kompetenz zu überprüfen.

\subsection{Experimentelle Bedingungen: Fremdsprachenunterricht in einer berufsorientierten Hochschule (am Beispiel einer beruflichen Militäreinrichtung in Russland)}

Das Experiment zur Entwicklung interkultureller Kompetenz im Fremdsprachenunterricht wurde im Ost-Sibirischen Institut des Ministeriums des Inneren (Восточно - Сибирский институт Министерства Внутренних дел Российской Федерации) in Russland durchgeführt. Das Institut stellt eine staatliche Bildungseinrichtung dar, wo Mitarbeiter des Innenministeriums ausgebildet und weiterqualifiziert werden. Die Besonderheit dieser Einrichtung im Vergleich zu anderen staatlichen Hochschulen besteht darin, dass die Studierenden nicht nur eine Ausbildung bekommen und einen Beruf erlernen, sondern zugleich Militärdienst leisten. Die Studierenden des Instituts haben seit Anfang ihres Studiums den Status 
von Staatsbeamten, und nehmen parallel zum Studium an den militärischen Veranstaltungen teil (z.B. Nacht- oder Küchendienst, Sicherung öffentlicher Veranstaltungen). Die Studierenden des Instituts werden „Kursanten“ genannt. ${ }^{52}$

Zur Zeit des Experiments bestand das Institut aus drei Fakultäten, und zwar der Juristischen Fakultät, der Fakultät für Brandsicherheit und der Fakultät für Fernstudien. Das Experiment zur Entwicklung interkultureller Kompetenz wurde an der Juristischen Fakultät durchgeführt. Daran nahmen die Kursanten der zwei Fachrichtungen teil: 0203 „Rechtsschutztätigkeit“ (mittlere Berufsausbildung) und 023100 „Rechtsschutztätigkeit: Qualifikation - Jurist" (höhere Berufsausbildung). Die Kursanten der mittleren Stufe studieren insgesamt vier Jahre (die Ausbildung in dieser Fachrichtung ist zweistufig aufgebaut: zwei Jahre und sechs Monate davon verbringen die Kursanten an der Hochschule, die restlichen 18 Monate absolvieren sie die Ausbildung als Fernstudium) und werden danach in den Rechtsschutzorganen als Abschnittsinspektoren oder Ermittlungsbeamte angestellt. Die Kursanten der höheren Stufe studieren fünf Jahre und werden später als Untersuchungsbeamte oder Ermittler eingesetzt.

Die Ausbildung an einer russischen Hochschule oder an einer Universität richtet sich nach zwei Kriterien: Zum einen ist es die Fortsetzung der schulischen Allgemeinbildung, zum anderen sind fachbezogene Fächer eingeplant, die den Studierenden auf seine berufliche Tätigkeit vorbereiten. Nach diesem System ist auch die Ausbildung am Ost-Sibirischen Institut des MdI aufgebaut. Im ersten Studienjahr liegt der Schwerpunkt der Ausbildung bei den allgemeinbildenden Fächern wie Mathematik, Russisch, Philosophie, Geschichte, Fremdsprache etc., in den weiteren Studienjahren verlagert er sich auf die berufsbezogenen Fächer wie Straf-, Strafprozess-, Zivil-, Arbeits-, Familienrecht etc. Die Auswahl der Fächer ist nicht frei, sondern streng von den Anforderungen des Programms reglementiert.

Eine Fremdsprache gehört zu den allgemeinbildenden Fächern und wird in der Fachrichtung „Rechtsschutztätigkeit“ in den zwei ersten Semestern, in der Fachrichtung „Jura“ in den ersten drei Semestern unterrichtet. Zur Auswahl stehen zwei Fremdsprachen: Deutsch und Englisch. In den meisten Fällen wird von dem/der Studierenden die Fremdsprache ausgewählt, die er/sie in der Schule erlernt hat.

Das Experiment wurde in den Gruppen des ersten Studienjahres durchgeführt, und zwar im Laufe des ersten Semesters. Das bedeutet, dass die allgemeinbildenden Themen dem Studienplan entsprechend im Mittelpunkt des Lernens gestanden haben.

52 Da es sich beim Ost-Sibirischen Institut um eine militärische Einrichtung handelt, sind die Kursanten verpflichtet, innerhalb der Hochschule eine Uniform zu tragen. Die männlichen Kursanten, die die Mehrheit der Studierenden bilden, sind im ersten und zweiten Studienjahr kaserniert. 


\subsubsection{Professionelle Kompetenz als übergeordnetes Ziel der berufsbezogenen Ausbildung.Die Bedeutung des interkulturellen Lernens für den weiteren beruflichen Werdegang der Auszubildenden}

Die Teilnehmer des Experiments erlernen in der Hochschule in erster Linie ihren zukünftigen Beruf. Die Entwicklung der professionellen Kompetenzen und das Beherrschen der Fachkenntnisse stehen im Mittelpunkt ihrer Ausbildung. Da ein relativ kompliziertes Auswahlverfahren für die Immatrikulation in die Ost-Sibirischen Hochschule der MdI vorausgeht, ist die Entscheidung für diesen Beruf den meisten Kursanten durchaus bewusst und verinnerlicht. Sehr viele setzen die Tradition ihrer Väter oder älteren Brüder fort und möchten Milizangehörige in der zweiten oder dritten Generation werden. Zwei weitere Faktoren kennzeichnen die Einstellungen der Kursanten. $\mathrm{Zu}$ einem sind sie bestrebt, den Beruf zu erlernen, weil sie danach einen sicheren Platz in den vom Staat finanzierten Verwaltungsorganen bekommen, was in der wirtschaftlich immer noch unsicheren Situation in Russland von Vorteil ist. Zum anderen lockt das Studium an dieser militärischen Hochschule durch für andere Hochschuleinrichtungen untypische Reize wie Uniform, Schießübungen oder Wachestehen. Aus der eigenen Erfahrung der Verfasserin lässt sich feststellen, dass die Kursanten des ersten Studienjahres motiviert und auf ihre beruflichen Ziele konzentriert sind.

Die während dieser Ausbildung angestrebten beruflichen Ziele sind als Anforderungen an zukünftige Juristen in den „Staatlichen Ausbildungsstandards“ für die Fachrichtungen 023100 und 0203 zusammengefasst. Unter anderem sind folgende Punkte zu finden:

1. Ein Jurist muss die Bürgerrechte, das Gesetz und die sozialen Werte eines Rechtsstaats akzeptieren, seine Berufspflicht erfüllen und sich gemäß den Normen der Berufsethik verhalten.

2. Ein Jurist muss den unbestrittenen Vorrang solcher Werte wie Recht, Freiheit und gesetzliche Interessen der Bürger, Rechtsordnung und Disziplin anerkennen.

3. Ein Jurist muss die Fähigkeit besitzen, die Sicherheit einer Person, die öffentliche Ordnung und die öffentliche Sicherheit zu gewährleisten.

4. Ein Jurist muss die Zusammenarbeit mit Mitarbeitern anderer Staatsorgane, mit Vertretern der Selbstverwaltung, öffentlicher Vereine, der Gewerkschaften, mit Bürgern und mit Medien organisieren.

5. Ein Jurist muss die Fähigkeit zur Kommunikation mit Bürgern verschiedener Schichten besitzen. 
Das heißt, professionelles Verhalten zukünftiger Juristen verlangt Werte wie Recht, Freiheit, Schutz der gesetzlich berechtigten Interessen der Bürger und der öffentlichen Sicherheit. Diese Werte sind auch für die interkulturelle Ausbildung wichtig. Als eine für diesen Beruf wichtige Kompetenz wird auch die kommunikative Fähigkeit hervorgehoben, die bei der Arbeit sowohl mit öffentlichen Institutionen wie mit Bürgern unterschiedlicher Schichten vonnöten ist. Es wird in diesem Fall von muttersprachlicher kommunikativer Kompetenz gesprochen.

Obwohl diese Anforderungen an zukünftige Juristen interkulturell wichtige Werte und kommunikative Kompetenz als Ausbildungsziel verlangen, ist die Notwendigkeit, die interkulturelle Kompetenz zu entwickeln, in den „Staatlichen Ausbildungsstandards“ nicht erwähnt. Es stellt sich daher die Frage, ob der interkulturellen Kompetenz die richtige Bedeutung eingeräumt wird

Die professionelle Tätigkeit der Kursanten des Ost-Sibirischen Instituts des MdI ist mit der Aufrechterhaltung der öffentlichen Ordnung und der Aufklärung von Straftaten verbunden. Die staatlichen Beamten, und dazu gehören auch die Juristen, sind diejenigen, die ständig mit unterschiedlichen Schichten unserer mulikulturellen Gesellschaft zu tun haben. Außer mit alltäglicher Kleinkriminalität sind sie auch mit illegaler Migration und mit Feindseligkeit gegen Ausländer konfrontiert.

Im Jahre 2003 wurden 463 Verbrechen auf dem Territorium des Irkutsker Gebiets von ausländischen Bürgern begangen. Als die beiden häufigsten Ursachen werden die Fälschung von Ausweisen und Drogenkriminalität genannt. Die Personen, die eine gesetzeswidrige Handlung begangen haben, kommen am häufigsten aus Republiken der ehemaligen Sowjetunion: Tajikistan, Azerbaijan, Uzbekistan. Ein weiteres Problem für die juristischen Organe ist die illegale Migration. Die geografische Lage des Irkutsker Gebiets ${ }^{53}$ macht es zu einer wichtigen Station für einreisende Migranten aus asiatischen Ländern. Nach Angaben des Migrationsdienstes halten sich jährlich etwa 10- bis 12.000 illegale Migranten auf dem Territorium des Gebiets auf. Das sind Gastarbeiter oder Flüchtlinge aus der Mongolei, China, Vietnam, Nord- und Südkorea, Afghanistan). (vgl. Irkutsker Forschungszentrum für organisierte Kriminalität o.J.)

Um alle diese Probleme bewältigen zu können brauchen Juristen etwas mehr als kommunikative Fähigkeit. Hier sind Fähigkeiten im interkulturellen Bereich, und zwar in erster Li-

53 Das Irkutsker Gebiet liegt in der Nähe der Grenze zur Mongolei und zu China. Die Transsibirische Einsenbahn, in dieser Region das wichtigste Verkehrsmittel sowohl für die Beförderung von Personen als auch von Waren, verläuft durch das Gebiet, dabei dient der Eisenbahnstation „Irkutsk“ als der wichtigste Verbindungsknoten. 
nie interkulturelle Sensibilität nötig.

In der Sowjetunion, wo Internationalismus großgeschrieben wurde, wurden die Bürger im Sinne der Gleichberechtigung aller Völker und in Anerkennung des interkulturellen Gedankens erzogen. Das Problem war m.E., dass die tatsächlich existierenden Unterschiede zwischen Kulturen, politischen Systemen, Wertsystemen etc. ignoriert wurden. Solche Einstellungen hatten gute Chancen, im relativ starren System der militärischen Einrichtung zu überleben. Ein Beamter, der sich für einen ausländerfreundlichen Mitarbeiter des öffentlichen Dienstes hält, schließt häufig die Augen vor den tatsächlich existierenden kulturellen Unterschieden. Bei seiner Arbeit unternimmt er keinen Versuch zu verstehen, was eigentlich der ausländische Bürger oder der Bürger mit Migrationshintergrund denkt und fühlt. Solchen Mitarbeitern fehlt bei allem guten Willen die Sensibilität gegenüber anderen Kulturen.

Es wird aber immer öfter Mitarbeitern der MdI Feindseligkeit gegenüber ausländischen Mitbürgern vorgeworfen. So beklagt die Zeitung „Novie Izvestija“ vom 28.09.2006, dass es niemanden gibt, der ,gegen das Anwachsen der aggressiven nationalistischen Bewegung kämpfen wird, da immer mehr Mitarbeiter der Rechtsorgane die Einstellungen der nationalistischen Organisationen teilen“. Im Grunde genommen werden diejenigen, die die Einhaltung von Gesetzen und mit deren Hilfe Ordnung und Frieden auf den Straßen zu schützen haben, selbst zum Auslöser nationaler Konflikte. Aufgrund der eigenen Einstellungen ermitteln Mitarbeiter der Milizorgane nicht mit voller Kraft bei Verbrechen mit nationalistischem Hintergrund. Außerdem werden nationalistische Organisationen nicht nur erlaubt, sondern sogar von den Personen, die an der Macht stehen, unterstützt (vgl. Zubtschenko und Pankow 2006).

Um solche schweren Vorwürfe der Unprofessionalität und Voreingenommenheit zu vermeiden, muss schon bei der Ausbildung zukünftiger Mitarbeiter der Milizorgane mehr Wert gelegt werden auf die Entwicklung solcher Werte wie Toleranz, Offenheit und Respekt im Umgang mit anderen Kulturen, weltoffene Haltung, ehrliches Interesse am fremdkulturellen Partner, also das, was in dieser Arbeit unter der affektiven Komponente der interkulturellen Kompetenz verstanden wird. Es ist aber auch wichtig, dass solche Themen wie interkulturelle Beziehungen noch während des Studiums besprochen werden, da das Veränderungspotenzial junger Menschen größer ist als das Erwachsener. Dies hilft auch, mögliche Konflikte innerhalb des multinationalen Kursantenkreises zu vermeiden.

Die derzeit in verschiedenen Weltteilen geführten Kriege haben auch ihre Auswirkungen auf dieses Problem. Als UNO-Mitglied nimmt Russland zusammen mit den anderen Mit- 
gliedsstaaten an Maßnahmen teil, die sich auf die Beilegung zwischennationaler Konflikte außerhalb des Territoriums der Russischen Föderation richten. Während solche Einsätze früher einen eher gelegentlichen Charakter hatten und keine große Zahl von Einsatzkräften forderten, änderte sich ihre Dynamik in den letzten Jahren grundsätzlich. Es werden nicht nur Streitkräfte gebraucht, die die beiden Kontrahenten eines zwischennationalen Konfliktes auseinanderbringen können. Es werden zurzeit vor allem interkulturelle Mediatoren gebraucht, die einen Konflikt an der Ausbreitung hindern und beilegen können. Das stellt ganz andere Anforderungen an die Vorbereitung der Einsatzkräfte.

Die nächste mögliche Situation, in der interkulturelle Fähigkeiten zukünftiger Mitarbeiter des MdI vonnöten sein könnten, ist mit den bewaffneten Konflikten im Kaukasus verbunden. In den Republiken Tschetschenien oder Ossetien existieren z.B. mehrere Konflikte, die nationale, politische oder religiöse Hintergründe haben oder als Machtkämpfe zwischen verschiedenen Clans betrachtet werden können. Regelmäßig werden die Mitarbeiter der russischen Miliz in sogenannte „Kampfdienststreifen“ gesandt. Bei solchen Streifen kommen sie fast immer mit der Zivilbevölkerung in Kontakt. Das bietet schon in friedlichen Zeiten ein weites Feld für Missverständnisse, im Krieg aber wird dies zu einem „Minenfeld“, das lebensbedrohende Situationen mit sich bringt, deren Ausgang sowohl für die Zivilbevölkerung als auch für die Einsatzkräfte unberechenbar ist.

Fazit: In der zukünftigen beruflichen Tätigkeit der Kursanten des Ost-Sibirischen Instituts des MdI wird es immer häufiger Situationen sowohl unmittelbar am Arbeitsplatz als auch während der Dienstreisen geben, die einen korrekten und respektvollen Umgang mit Menschen anderer kultureller und nationaler Herkunft verlangen. Aus diesem Grund muss die interkulturelle Kompetenz der Milizmitarbeiter ein Teil ihrer beruflichen Kompetenz werden, d.h. sie muss einen bedeutenden Platz in ihrer Ausbildung finden.

\subsubsection{Ziele und Rahmen des Fremdsprachenunterrichts}

Das Erlernen einer Fremdsprache in einer berufsorientierten Hochschule ist ein Teil des Pflichtprogramms. Das Ziel des Fremdsprachenunterrichts ist dem Ziel des gesamten Studiums untergeordnet, und zwar der Ausbildung eines Studierenden für einen Beruf und seine Vorbereitung für die zukünftige berufliche Tätigkeit. Das Ziel des Fremdsprachenunterrichts wird wie folgt formuliert:

„Das Hauptziel des Fremdsprachenunterrichts in der modernen Zeit ist die Entwicklung 
der fremdsprachlichen kommunikativen Kompetenz. Darunter wird ein bestimmtes Niveau sowohl der Sprachbeherrschung als auch der Entwicklung der soziokulturellen Kenntnisse, Fähigkeiten und Fertigkeiten verstanden, die den Lernenden erlauben, adäquat und gezielt ihr Sprachverhalten zu variieren. Entsprechend dem Programm für die Hochschulen für nichtsprachliche Berufsfachrichtungen hat ein Fremdsprachenkurs einen kommunikativen und einen berufsorientierten Charakter. Das bedeutet, dass ein Fremdsprachenkurs in einer Hochschule die Beherrschung von Kenntnissen, Fähigkeiten und Fertigkeiten zu gewährleisten hat, die dem Lernenden erlauben, die interkulturelle Kommunikation im Bereich der professionellen Tätigkeit zu führen. Die professionelle Tätigkeit wird durch die Fachrichtung der Hochschule bestimmt“ (Pjantschina, Mosejtschuk und Paschkowa 2009).

Der gesamte Kurs einer Fremdsprache im Ost-Sibirischen Institut der MdI ist zweistufig aufgebaut. Im ersten Teil wird der Schwerpunkt auf allgemeine Themen gelegt wie „Das bin ich“, „Meine Familie“, „Zeit und Tagesablauf“, „Meine Hochschule“, „Hallo Deutschland“. Als Hauptziel wird dabei die kommunikative Kompetenz der Studierenden hervorgehoben. Es wird die Fähigkeit entwickelt, sowohl monologische Aussagen zu personenbezogenen, individuellen Themen zu machen als auch Gespräche zu diesen Themen zu führen, bei denen Regeln eingehalten werden. In diesem Kursteil werden die lexikalischen und grammatikalischen Kenntnisse wiederholt und systematisiert, die im Schulunterricht schon erlernt wurden. Sie bilden auch den Grundstein für weitere Themen.

Der zweite Teil des gesamten Kurses hat schon eine berufliche Orientierung. Neben der kommunikativen Kompetenz wird hier auch eine Übersetzungskompetenz entwickelt. Der Schwerpunkt liegt auf berufsorientierten Themen wie „Am Tatort“, „Im Dienst der Sicherheit“, „Die deutsche Polizei“, „Internationale polizeiliche Arbeit“. Es wird die Fähigkeit entwickelt, Fachtexte zu verstehen und zu übersetzen. Die Schwierigkeit dieser Texte besteht darin, dass sie oft in einer Fachsprache geschrieben sind, die durch spezielles Vokabular und schwierige grammatische Strukturen charakterisiert ist. Da aber die kommunikative Kompetenz auch hier als Ziel des Unterrichts gilt, wird in ihrem Rahmen die Fähigkeit entwickelt, Gespräche mit berufsorientierten Themen zu führen.

Die Zahl der Unterrichtsstunden pro Woche variiert gemäß dem Stundenplan, der jede Woche neu zusammengestellt wird. Im Durchschnitt sind es zwei bis drei Doppelstunden pro Woche für beide Fachrichtungen, die an dem Experiment beteiligt waren.

Prüfungen.

Laut den staatlichen Ausbildungsstandards für die mittlere Berufsausbildung vom 01.09.2002 beträgt die Gesamtzahl der Unterrichtsstunden in einer Fremdsprache für die 
Fachrichtung 0203160 Stunden. Diese Stunden erstrecken sich über zwei Semester. Das Studienfach „Fremdsprache“ ist im Modul „Allgemeine humanitäre und sozial-wirtschaftliche Fächer“ eingeschlossen. Die Anforderungen sind im Standard wie folgt formuliert:

1. Grundlagen einer Fremdsprachenkommunikation: Phonetik, Lexik, Phraseologie und Grammatik;

2. Grundlagen einer Fachsprache: fachorientierter Wortschatz, Phraseologie und Fachausdrücke;

3. Übersetzungstechniken von beruflich orientierten Texten mit dem Wörterbuch; Fachkommunikation.“

Laut den staatlichen Ausbildungsstandards für die höhere Berufsausbildung vom 08.02.2001 ist die Gesamtzahl der Unterrichtsstunden im Fach „Fremdsprache“ für die Fachrichtung 023100 auf 340 Stunden festgelegt. Die Stunden sind auf vier Semester verteilt. Unten wird das inhaltliche Minimum des Studienfaches „Fremdsprache“ angeführt:

„Die Spezifika der Lautartikulation, der Intonation, der Betonung und des Rhythmus in der zu erlernenden Sprache; Besonderheiten der Aussprache, die für die Fachkommunikation charakteristisch sind; Transkriptionsregeln; lexikalisches Wortschatzminimum von 4000 Spracheinheiten der allgemeinen und berufsorientierten Themen; Vorstellung über die Einordnung des Wortschatzes nach Anwendungsbereichen (wie z.B. alltäglich, wissenschaftlich, professionell, offiziell etc.); Vorstellung über freie und feste Wortverbindungen und phraseologiesche Redewendungen; Wortbildung; grammatikalische Kenntnisse, die die allgemeine Kommunikation ohne Sinnverzögerrung in der mündlichen und in der schriftlichen Form gewährleisten; grammatische Erscheinungen, die für die Fachtexte charakteristisch sind; Vorstellung über die Alltags-, Wissenschafts-, Fachsprache und Literaturstile; Landeskunde und Traditionen der Länder, wo die zu erlernende Fremdsprache im Alltag gesprochen wird, Sprachetikette; dialogische und monologische Aussagen in einigen offiziellen und nicht offiziellen kommunikativen Situationen; Grundlage einer öffentlichen Aussage (z.B. Vortrag); Hörverstehen in den Bereichen Alltags- und Fachkommunikation Leseverstehen von Texten pragmatischen und berufsorientierten Ursprungs; angemessener schriftlicher Ausdruck bei Gutachten, Referat, Thesen, Mitteilung, privatem oder formellem Brief, Lebenslauf.“ ( Bildungsministerium der Russischen Föderation 2001a)

Aus den beiden Auszügen ist zu ersehen, dass das Programm für eine Fremdsprache in der berufsorientierten Hochschule alle vier vom Europäischen Gemeinschaftsrahmen festgelegten Fertigkeiten einschließt: Hörverstehen, Leseverstehen, schriftlicher Ausdruck und mündliche Kommunikation. Dabei werden zwei von diesen Bereichen deutlich bevorzugt, 
und zwar Leseverstehen und Sprechen.

Die Fortschritte der Kursanten werden kontrolliert. Innerhalb des Semesters werden bei Abschließen eines Themas Kontrollarbeiten durchgeführt. Am Ende jedes Semesters finden Prüfungen statt, dabei wird nur die letzte am Ende aller Unterrichtsstunden mit einer Note bewertet.

\subsubsection{Unterrichtsmaterial und Lehrwerk}

Die interkulturellen Inhalte des PEIK sind als Zusatzmodule zu einem regulären Fremdsprachenunterricht gedacht. Jeder Unterricht stützt sich normalerweise auf ein oder mehrere Lehrwerke, die den Anforderungen des Programms und den Zielen des Fremdsprachenlernens in einer bestimmten (Hoch-)Schule entsprechen. In diesem Kapitel wird die Frage behandelt, ob das in den experimentellen Gruppen eingesetzte Lehrwerk den interkulturellen Gedanken nachweislich fördert.

Für den Zeitraum des Experiments wurde ein für diese Fakultät neues Lehrbuch „Deutsch für zukünftige Brandschutzingenieure“ (Pjatschina 2002) eingesetzt. Dieses Buch löste ein anderes Lehrwerk, und zwar „Deutsch. Lehrwerk für die Hochschulen des MdI Russland“ von Maslowa, Slusarenko, Bisgeimer und Gorbunowa (1998) ab. Das neue Buch wurde von einem russischen Autorenkollektiv zwar für eine andere Fakultät verfasst, enthielt aber alle allgemeinen Themen, die vom Lehrprogramm vorgeschrieben wurden. Wie schon in Kapitel 6.2.1. erwähnt, fand das Experiment im ersten Semester statt, d.h. es wurden lediglich allgemeine, nicht berufsorientiere Themen behandelt. Daher war es möglich, dieses neue Lehrbuch als Hauptlehrwerk im Unterricht einzusetzen ${ }^{54}$.

Der interkulturelle Gedanke basiert auf dem Bewusstsein der Unterschiede zwischen der fremden und der eigenen Kultur und auf der Akzeptanz des Fremden als Normalität. Bei der Analyse des Lehrwerkes wurde die Frage gestellt, ob die angebotenen Themen für die Arbeit an dem interkulturellen Gedanken tauglich sind und ob sie den Lernenden eine positive Öffnung für das Fremde ermöglichen (vgl. Volkmann 1999, S. 122). Analysiert wurden der Einführungskurs und die ersten drei Kapitel des Lehrwerks (,Willkommen in unserer Hochschule“, „Beruf und Arbeit“, „Hallo Deutschland“), da genau diese während des Ex-

54 Auf der Grundlage des Lehrbuches „Deutsch für zukünftige Brandschutzingenieure“ wurde ein weiteres Lehrbuch „Deutsch für zukünftige Milizbeamte“ des (fast) gleichen Autorenkollektivs einige Jahre später ausgearbeitet. So konnten zugleich die allgemeinbildenden Themen aus dem Lehrbuch für die Brandschutzingenieure auf ihre Tauglichkeit auch für den Unterricht in der Juristischen Fakultät geprüft werden. 
periments im Unterricht eingesetzt wurden.

Die Normen und Kriterien für die Analyse des Lehrwerkes wurden aus den Artikeln von Volkmann (1999) und Abendroth-Timmer $(1997,1999)$ entnommen. Zur Erfassung der impliziten und expliziten Normen und Werte in Lehrwerken schlägt Volkmann vier Komplexe vor:

- Selektionsfrage: „Wie steht es mit Selektion, Räpresentation, Stereotypik?““

- Homogenisierungsmethoden vs. Differenzstruktur: „Werden homogenisierende Vorstellungen bevorzugt? Oder wird Differenz betont und geschaffen?“

- Wertung und Konfiguration der Lebensformen: „Besteht die Koexistenz von unterschiedlichen Lebensformen?“”

- Ausgewogenheit der Elemente: „Wie steht es mit dem ,ausgewogenen“ Verhältnis der einzelnen Elemente?“ (vgl. Volkmann 1999)

Diese Fragen lassen sich anhand von bestimmten ausgewählten Kriterien operationalisieren. Bei der Analyse des eingesetzten Lehrwerkes ergaben sich anhand der oben angeführten Kriterien folgende Schlussfolgerungen:

\section{Länder, Regionen, Raum}

Die alte Vorstellung, dass die Sprache einer festen kulturellen Gemeinschaft zugehört, entspricht nicht dem interkulturellen Gedanken. Die nationale Kultur versteht sich als Bund von mehreren Gemeinschaften und Individuen. Im Lehrbuch ist aber eindeutig zu sehen, dass der Ausdruck ,in Deutschland“ bevorzugt wird. Es mangelt an Ausgewogenheit bezüglich der Informationen über andere deutschsprechende Länder wie Österreich, die Schweiz, Luxemburg oder Liechtenstein. Selbst Deutschland wird als ein einheitliches Konzept verstanden, ohne dass regionale Unterschiede oder Unterschiede zwischen der Stadt und Land zum Ausdruck gebracht werden.

\section{Ethnische Gruppen}

Da im Lehrbuch Deutschland als ein einheitliches Konzept behandelt wird, wird auch die ethnische Zusammensetzung des Landes nicht hervorgehoben. Leider sind keine Hinweise auf die zahlreichen Bevölkerungsgruppen zu finden wie Sachsen, Hessen, Bayern etc, die innerhalb des Landes leben, dabei aber oft unterschiedliche Traditionen, Dialekte und Bräuche haben. Es gibt auch keine Versuche, die Beziehungen zwischen den ehemaligen ost- und westdeutschen Bürgern anzusprechen. Das einzige Thema, das in Bezug auf nationale Minderheiten aufgegriffen wurde, sind die in Deutschland lebenden Ausländer. Aussagen über sie haben meist informativen Charakter, dabei wird auch versucht, die Binnenper- 
spektive der Bürger mit Migrationshintergrund zu zeigen, was Verständnis und Sympathie für solche Gruppen weckt (vgl. S. 37, 142-144). Es werden keine klischeehaften Charakterisierungen vermittelt, nationale Stereotype werden zum Teil aufgedeckt (vgl. S. 142-146). Darüber hinaus wird jedoch nicht das Bild einer multikulturellen Gesellschaft vermittelt, die von ethnischer Vielfalt geprägt ist. Ausländer mit ihren Einstellungen werden eher als Außenseiter präsentiert und den imaginären Deutschen gegenübergestellt.

\section{Geschlechterrollen/Geschlechterdifferenz}

Genauso wenig Platz nehmen die feministischen Ideen in diesem Lehrbuch ein. Die Geschlechterrollen sind traditionell zwischen Mann und Frau verteilt, wie z.B. beim Thema „Zeit- und Tagesablauf“ (S. 58), wo eine Mutter Frühstück macht, Kinder in den Kindergarten bringt und der Vater lediglich zur Arbeit geht. Eine ähnliche Situation wird auch beim Thema „Beruf und Arbeit“ beobachtet, wo frauentypische Berufe wie Krankenschwester oder Sekretärin den durch die Männer besetzten Berufen wie Pilot, Feuerwehrmann, Polizist gegenübergestellt sind (Bild S. 111). Die patriarchalen Muster haben sich auch in der Sprache durchgesetzt, indem weibliche Pronomina und Wortbildungen nicht gebraucht wurden (Bürokaufmann, aber keine Bürokauffrau; Feuerwehrmann, aber keine Feuerwehrfrau etc.) Die Genderproblematik wird nicht thematisiert, genauso wenig werden die Leser des Lehrbuches für die neuen Konstrukte von Männlichkeit oder Weiblichkeit in der modernen Gesellschaft sensibilisiert.

\section{Kulturelle Praktiken}

Da dieser Aspekt breitgefächert ist, ist die Zusammenfassung der Analyse kompliziert. Nach der Meinung der Verfasserin fehlt diesem Lehrbuch ein einheitliches Konzept, dessen Ziel es sein soll, die Fremdkultur als breite Palette unterschiedlicher Alltagspraktiken bewusst zu machen. Da die Texte und Übungen aber aus authentischen Quellen entnommen wurden, tauchen Differenzen und Kontraste des Alltags in Deutschland in unterschiedlichen Themenbereichen auf. So wird z.B. beim Thema „Hallo Deutschland“ die Binnenund die Fremdperspektive beim Blick auf Deutschland präsentiert, wobei die Diskussion über typische/nicht typische Angewohnheiten und der Vergleich mit der eigenen Kultur angeregt wird. Zum gleichen Thema ist aber auch der Text über Weihnachten zu finden, der alle Deutschen pauschalisiert, dabei entsteht der Eindruck der Gleichheit und der Ähnlichkeit. Dieser Eindruck wird auch in den weiteren Übungen nicht bearbeitet. Meistens tragen die Texte und Übungen einen informativen Charakter, es werden aber auch ab und zu einige Aspekte zwischenmenschlicher Beziehungen thematisiert, wie z.B. in der Übung (S. 47) zum Thema „Meine Familie“ mit der Frage „Was ist für Sie eine Familie?“ und den Multi- 
ple-Choice-Antworten oder dem Infopoint „So leben junge Erwachsene“ (S. 52). Dieses Bild ignoriert die Tatsache, dass ein „Mittelklassenleben“ als ein implizites Ideal in diesem Lehrbuch zu finden ist, wobei versäumt wird, Unterschiede und Verhältnisse zwischen der Elitekultur und der Massenkultur darzustellen.

\section{Kommunikation}

Dialoge finden sich in deutlich geringerer Anzahl als Texte und Übungen. Die Kommunikation darin ist sehr formell aufgebaut, passt meistens eher zum gedankenlosen Auswendiglernen, als dass sie zum eigenen Denken anregt. Die Dialoge haben meist eine geschlossene Struktur und lassen keinen Platz für Variationen. Auf Seite 151 wird das Thema „,nonverbale Kommunikation“ eingebracht, indem die auf den Bildern dargestellten Gesten von Deutungsbeschreibungen begleitet sind. Es sind aber keine weiteren Infospots bezüglich der Höflichkeitsnormen oder Kommunikationsregeln zu finden.

\section{Textvarianten und Textkorrelation}

Bei den Textvarianten geht es um die Authentizität und die Repräsentativität der Texte. Mit wenigen Ausnahmen sind die Texte in dem analysierenden Lehrbuch authentisch. Sie präsentieren meistens Erscheinungen aus dem Leben junger Menschen, deshalb werden die Texte und ihre Sprache dem Alter und den Interessen der Studierenden gerecht. Die die Texte begleitenden Bilder (gezeichnet, nicht farbig) decken sich nur zum Teil mit den Aussagen der Texte. In anderen Fällen entsteht ein Spannungsverhältnis zwischen den Informationen aus dem Bild und den Inhalten des Textes, indem verschiedene Meinungen über ein Problem dargestellt werden, was die Studierenden zum Nachdenken und zu Diskussionen animiert. Solche Darstellung von Vielfalt ist z.B. im Spannungsverhältnis zwischen dem Text über die Familie Bosse (S. 51) und dem bildlich dargestellten Infopoint „So leben junge Erwachsene“ (S. 52) zu beobachten. Ein gutes Zeichen für die Tauglichkeit des Lehrbuches hinsichtlich des Ziels „Interkulturelle Kompetenz“ ist, dass der Inhalt der Texte meistens eine Problematik aufwirft, die später zu einer Kommunikation anregen soll. Es sind die eigenen Meinungen der Kursteilnehmer zum jeweiligen Thema gefordert, was den kreativen Umgang mit den Texten fördert. Zusätzlich wünschenswert sind aber Fragen, in denen der Bezug zur eigenen Kultur der Teilnehmer aufgenommen wird.

Ein Teil der Texte ist allerdings nicht authentisch. Dies betrifft das Thema „Willkommen in unserer Hochschule“, wo sowohl einige Texte als auch einige Dialoge künstlich gemacht sind. Diese Texte haben einen Beschreibungscharakter, sind geschlossen in ihrer Struktur und regen eindeutig zum Auswendiglernen an.

Sprache 
Das Lehrbuch ist zweisprachig aufgebaut. Die Aufgabenstellungen werden am Anfang des Buches auf Russisch und erst ab dem Thema „Willkommen in unserer Hochschule“ auf Deutsch geschrieben. Außerdem gibt es zu jedem Thema eine Wortschatzliste mit deutschrussischer Übersetzung. $\mathrm{Ab}$ und $\mathrm{zu}$ sind auch einige Informationen bezüglich grammatischer und kultureller Erscheinungen auf Russisch $\mathrm{zu}$ finden. Diese und einige andere Merkmale, wie z. B. nicht authentische Texte sind der lernerorientierten Funktion ${ }^{55}$ als Lehrbuch zuzuschreiben.

\subsection{Planung und Durchführung des Experiments}

Das Experiment zur Erprobung des PEIK im Fremdsprachenunterricht fand im ersten Semester (Wintersemester) des Studienjahres 2004/2005 statt. Das Programm wurde als Ergänzung zum geplanten Fremdsprachenunterricht in zwei Gruppen des ersten Studienjahres von September 2004 bis Februar 2005 eingesetzt. Zur Evaluation der Wirksamkeit von PEIK wurde vor der ersten Sitzung ein Prätest und nach der letzten Sitzung ein Posttest durchgeführt. Prä- und Posttests wurden ebenso in zwei Kontrollgruppen durchgeführt, die innerhalb des oben genannten Zeitrahmens einen traditionellen Fremdsprachenunterricht erhielten.

Das Experiment wurde mit dem Einverständnis der Leiterin des Lehrstuhls „Fremdsprachen und Sprachkultur“" Frau Prof. Dr. Pjatschina durchgeführt.

\subsubsection{Experimentelle Methode}

Zur Überprüfung der im Kapitel 6.1. formulierten Hypothese, dass das Niveau interkultureller Kompetenz der Lernenden steigt, wenn im Unterricht gezielt daran gearbeitet wird, den Lernenden kulturspezifische und kulturübergreifende Kenntnisse beizubringen, dabei ihr Bewusstsein für die Besonderheiten einer interkulturellen Situation erweckt wird und sie für diese Besonderheiten sensibilisiert sowie interkulturell bedeutsame Fähigkeiten entwickelt werden, wurde zugunsten der experimentellen Methode entschieden.

Bei einem psychologischen oder pädagogischen Experiment geht es darum, die Bedingungen zu variieren, um ihre Wirkung durch Vergleich der Versuchsergebnisse auf die zu un-

55 Der Lernende mit seiner Sprache und seinen Interessen steht im Mittelpunkt des Lernprozesses. 
tersuchenden Vorgänge zu prüfen.

Das Experiment zur Entwicklung der interkulturellen Kompetenz wird im Rahmen des Fremdsprachenunterrichts an einer Hochschule durchgeführt, findet also in einem speziellen Untersuchungsraum statt. Dabei ist es den Teilnehmern bewusst, dass sie den Bedingungen eines experimentellen Programms unterzogen werden. Es muss erwähnt werden, dass unterschiedliche Einflussfaktoren nicht ausgeschlossen werden können, z.B. dass Teilnehmer, die seit ihrer Kindheit emotionale Eigenschaften wie Empathie oder Offenheit gut entwickelt haben, bessere Möglichkeiten haben, diese Eigenschaften auf die „neuen“ interkulturellen Bedingungen zu übertragen und zugleich bessere Chancen, gerade in der Variable Sensibilität ihre Ergebnisse zu verbessern. Der Deutschunterricht findet zwei- bis dreimal mit einer Doppelstunde statt, außerhalb dieser Zeit studieren die Teilnehmer andere Fächer und leisten gleichzeitig einen Militärdienst. Das bedeutet, dass sie unter dem Einfluss zahlreicher anderer Faktoren stehen, die sich sowohl fördernd als auch störend auf das Ergebnis auswirken können.

Es ist in der Forschungspraxis kaum möglich, die Einflüsse aller Störfaktoren zu kontrollieren. So waren bei diesem Experiment die Versuchsgruppen bereits bestehende Studentengruppen, d.h. die Gruppenzuteilung der Versuchspersonen erfolgte nicht nach bestimmten Kriterien. Aufgrund dieser Tatsachen ist festzustellen, dass es sich hier, bezogen auf die Kontrolle der Störvariablen, um ein Quasi-Experiment handelt.

\subsubsection{Zielgruppendarstellungen}

Das Experiment wurde in zwei Gruppen der Juristischen Fakultät des Ost-Sibirischen Institut des MdI durchgeführt.

Die Kursanten der Gruppe $2141^{56}$ sind für die Fachrichtung 023100 „Rechtsschutztätigkeit: Qualifikation - Jurist“ (höhere Berufsausbildung) immatrikuliert. Die durchschnittliche Gruppe in dem oben genannten Institut besteht aus etwa 30 Kursanten, die im Fremdsprachenunterricht in zwei kleinere Gruppen aufgeteilt wird. EG1 wurde vom Anfang des Studienjahres an aufgeteilt, und zwar erlernte eine Gruppe die englische Sprache, die zweite Deutsch. Für die Aufteilung der Kursanten waren zwei Gründe maßgeblich: welche Sprache sie in der Schule erlernt hatten und ihr eigenen Wunsch. Im Weiteren folgt die Beschreibung der Gruppe, die den Unterricht in der deutschen Sprache erhielt und diesbezüg-

56 Im weiteren Textverlauf wird die Gruppe 2141 Experimentelle Gruppe 1 (EG1) genannt. 
lich am Experiment teilnahm. Sie bestand aus 14 Kursanten, darunter 12 Männer und zwei Frauen. Die geringere Zahl der weiblichen Teilnehmer ist bei diesem Beruf in Russland nicht außergewöhnlich, so bilden nach den Beobachtungen der Autorin Teilnehmerinnen etwa 15-20\% aller Unterrichtsgruppen. In der Gruppe befanden sich 17- bis 21-Jährige, die meisten hatten im Juni 2004 ihr Abitur gemacht und nach den Aufnahmeprüfungen direkt mit dem Studium angefangen. 12 Teilnehmer hatten als Pflichtfach in der Schule Deutsch, zwei Französisch, d.h. im ersten Semester waren zwei Teilnehmer der Gruppe Anfänger. Nach den Fähigkeiten und den Leistungen der Teilnehmer kann EG1 nicht als homogen eingeschätzt werden: neben den Anfängern gab es Teilnehmer, die sich schon am Anfang des Semesters relativ frei zu einfachen Themen wie „Das bin ich“ oder „Familie“ äußern konnten. Diese Unterschiede können durch folgende Tatsachen erklären werden. Zum einen sind Fähigkeiten und die Motivation zum Fremdsprachenlernen bei den Teilnehmern unterschiedlich. Das spiegelt sich in der Note, die sie im Schulzeugnis nach Abschluss der Schule haben. Zum anderen spielt der Status der Schule nach Meinung der Verfasserin eine große Rolle. Die Abiturienten aus städtischen Gymnasien haben bessere Deutschkenntnisse als diejenigen, die ihre Ausbildung in kleineren Schulen aus abgelegenen Dörfern bekamen. Zum Ende des Semesters haben sich die Unterschiede etwas ausgeglichen, Homogenität wurde aber kaum erreicht. Um die Leistungen der Teilnehmer innerhalb des Semesters einzuschätzen, wurde eine Kontrollarbeit mit dem grammatischen und lexikalischen Stoff der erlernten Themen durchgeführt, die Durchschnittsnote der Gruppe betrug 3,9. ${ }^{57}$

Die Gruppe $2121^{58}$ nahm auch an dem Experiment teil. Ihre Kursanten gehören zu der Fachrichtung 0203 „Rechtsschutztätigkeit“ (mittlere Berufsausbildung). Die Aufteilung der Gruppe fand wie bei EG1 statt, die ,deutsche“ Gruppe bestand aus 16 Teilnehmern, darunter 15 Männern und einer Frau. Alle Teilnehmer dieser Gruppe hatten Deutsch in der Schule gelernt, und obwohl das Sprachniveau der Kursanten relativ homogen war, waren die Lernleistungen dieser Gruppe schwächer als die der EG1. Dabei spielte der zweite oben genannte Grund nach Meinung der Verfasserin gerade in diesem Fall eine entscheidende Rolle: die meisten Kursanten kamen aus entfernten Dörfern, wo der Sprachunterricht in den kleineren Schulen nicht die gleichen Möglichkeiten und nicht die gleiche Bedeutung hat wie in den städtischen Schulen. Das hat sich auch auf die Ergebnisse der Semesterkon-

\footnotetext{
57 In Russland ist folgendes Benotungssystem in Schulen, Fach-, Hochschulen und Universitäten üblich: Die besten Leistungen werden mit der Note ,5“ bewertet, was „,sehr gut“ entspricht. Weiter folgen: „,4“- „gut“, „3“- ,genügend“, „2“ - ,mangelhaft“. Die Note „,“ bedeutet „nicht ausreichend “, wird aber in der Unterrichtsrealität kaum gebraucht.

58 Im weiteren Textverlauf wird die Gruppe 2121 Experimentelle Gruppe 2 (EG2) genannt.
} 
trollarbeit ausgewirkt: EG2 hat mit der Durchschnittsnote 3,7 schlechter abgeschnitten als EG1. Die Teilnehmer sind zwischen 17 und 22 Jahre alt, die meisten machten vor Kurzem ihr Abitur. Es waren aber auch zwei ältere Kursanten dabei (21,22 Jahre alt), die schon ihren Armeedienst hinter sich hatten ${ }^{59}$. Einer davon hatte die Funktionen eines Gruppenkommandeurs ${ }^{60}$. Die Kursanten wurden zu diesem Posten vom militärischen Leiter des ersten Studienjahres ernannt. Als eine Besonderheit dieser Gruppe ist auch ihre nationale Gemischtheit zu nennen. Von 16 Teilnehmern sind fünf burjatischer Herkunft. Das hatte mehrere Konsequenzen: Bedeutsam sind z.B. andere kulturelle Einstellungen, aber auch, dass die Unterrichtssprache Russisch für einige burjatische Kursanten eine Zweitsprache war.

\subsubsection{Ablauf des Experiments}

Das Experiment wurde im Verlaufe von 28 Sitzungen durchgeführt, die folgende Inhalte hatten:

\begin{tabular}{|c|}
\hline Prätest \\
\hline $\begin{array}{l}\text { Einführung: Erklärung des Ziels der Programm, Definition der Begriffe „Interkultu- } \\
\text { relle Kompetenz“ und „Kultur“" sowie des Eisbergmodells der Kultur }\end{array}$ \\
\hline Fall: „Deutsch-brasilianisches Gespräch in der Firma“. Kulturassimilator-Methode \\
\hline Fall: „Private“ Kulturassimilatormethode \\
\hline Referat: Kulturtheorien von Holl, Hofstede \\
\hline Fall: „Polizei“. Methode des offenen Falls \\
\hline Fall: „Ein russischer Gast“. Methode des offenen Falls“ \\
\hline $\begin{array}{l}\text { Fallbeispiel aus den interkulturellen Erfahrungen des Lernenden. Methode: Analyse } \\
\text { des kritischen Ereignisses }\end{array}$ \\
\hline Fallbeispiel aus den interkulturellen Erfahrungen des Lernenden \\
\hline $\begin{array}{l}\text { Analyse des ausgewählten Fallbeispiels aus der interkulturellen Erfahrung eines } \\
\text { Lernenden. Methode des offenen Falls }\end{array}$ \\
\hline Checkliste zur Erfassung der kulturellen Identität \\
\hline Satzergänzungsübung zur kulturellen Selbstbefragung \\
\hline Fragebogen zur Erhebung von Kulturkontrasterfahrungen \\
\hline Kulturelle Simulation \\
\hline Durchführung des Fragebogens „,Deutschland und Deutsche“ \\
\hline
\end{tabular}

59 Jeder männliche Bürger zwischen 18 und 27 ist in Russland verpflichtet, einen zweijährigen Pflichtdienst in der russischen Armee abzuleisten. Es gibt keine Alternative zum Militärdienst. Nur aufgrund gesundheitlicher Faktoren kann ein Mann von diesem Dienst befreit werden. Ausnahmen stellen aber auch die Kursanten solcher Militäreinrichtungen wie das Ost-Sibirische Institut des MdI dar, wo die Kursanten gleichzeitig mit dem Studium ihren Militärdiest leisten. Immatrikulationswillige, die einen Armeedienst schon hinter sich haben, werden bei dem Auswahlverfahren bevorzugt.

60 Da es hier um eine Militäreinrichtung handelt, sind die Gruppen wie kleine militärische Einheiten gestaltet. D.h. ein Kursant ist von der Kursleitung als Gruppenkommandeur ernannt, die anderen Kursanten sind ihm unterordnet. 


\begin{tabular}{|l|}
\hline $\begin{array}{l}\text { Einführung ins Thema „Nationale Stereotype“. Diskussionsrunde „Deutschland und } \\
\text { Deutsche“ }\end{array}$ \\
\hline Arbeit am Thema „Andere Länder in unseren Köpfen“ \\
\hline Unterrichtseinheit „,Typisch Russisch?“ \\
\hline $\begin{array}{l}\text { Ein studentischer Vortrag zum Thema „Einheit der Verschiedenen“ über die Volks- } \\
\text { gruppen, Dialekte und Ausländer in Deutschland. Meinungsaustausch }\end{array}$ \\
\hline Spielstunde „Konferenz der Tiere“ \\
\hline Interkulturelle Sprachübungen „Spaghetti für zwei““ (Teil 1) \\
\hline Interkulturelle Sprachübungen „Spaghetti für zwei““ (Teil 2) \\
\hline Spielstunde „, Dill Dominoso“ \\
\hline Unterrichtseinheit „Kulturelle Regeln und Tabus in Minidialogen““ \\
\hline Unterrichtsstunde „Körpersprache““ \\
\hline Vergleichsanalyse eines Märchens „,Der Wolf und die sieben Geißlein““ \\
\hline Phraseologische Redewendungen. Vergleichsanalyse \\
\hline Diskussion „Mein Deutschland““ \\
\hline Arbeit an den Münchener Thesen zum interkulturellen Lernen \\
\hline Posttest \\
\hline
\end{tabular}

\subsection{Auswertung der Ergebnisse des Experiments}

Um Erfolge und Misserfolge des angewandten Programms evaluieren zu können, wurde vor Beginn des Experiments in einem Prätest und am Ende in einem Posttest das Niveau der interkulturellen Kompetenz der Teilnehmer gemessen. In dem folgenden Kapitel wird auf das Problem des Messinstruments für die interkulturelle Kompetenz eingegangen und der von der Verfasserin entwickelte Puzzle-Fragebogen präsentiert. Im Weiteren werden die angewandten Verfahren sowohl für die Datenanalyse als auch für die Überprüfung der statistischen Signifikanz beschrieben.

\subsubsection{Instrument zur Evaluierung der Ergebnisse}

Es existiert zurzeit kein anerkanntes Instrument zur Messung der interkulturellen Kompetenz. Moderne Untersuchungen basieren auf unterschiedlichen Modellen und haben meistens nur eine Seite der Kompetenz als Schwerpunkt. Da kein umfassendes theoretisches Konzept der interkulturellen Kompetenz vorliegt, ist es schwierig, ein einheitliches Messverfahren zu entwickeln. Dies bestätigt auch der Testkatalog 2008/09 der von der Testzentrale Göttingen anerkannten Tests, in dem kein Verfahren zur Messung der interkulturellen Kompetenz zu finden ist. Daher entschied sich die Verfasserin, ein Evaluationsinstrument 
in Anlehnung an den von Alvino Fantini im Jahre 2000 veröffentlichten „YOGA“-Test ${ }^{61}$ zu entwickeln. Dabei wurden die meisten Fragen zur Evaluierung von interkulturellen Kompetenzen dem „YOGA“-Test entliehen; der Aufbau des Instruments beruht jedoch auf dem von der Autorin entwickelten Puzzle-Modell. Im Weiteren wird dieses Instrument Puzzle-Fragebogen genannt. Zunächst folgen einige Überlegungen allgemeiner Art zum Untersuchungsverfahren, dann zum YOGA-Test und seiner bisherigen Verwendung.

Der Puzzle-Fragebogen ist ein Selbstevaluationsinstrument, d. h. die Teilnehmer schätzen selbst durch die Beantwortung vorgegebener Fragen das Entwicklungsniveau ihrer interkulturellen Kompetenz. An dieser Stelle muss erwähnt werden, dass die Selbstevaluation nach Meinung von Experten (vgl. Deardorff o.J., S. 31) nicht das einzige Instrumentarium zur Messung der interkulturellen Kompetenz ist. Andere Bewertungsmethoden sind Interviews, qualitative Messungen, Beobachtungen durch andere etc. Darüber hinaus ist die Effizienz von Prä-/ Posttestvergleichen unter Experten im interkulturellen Bereich umstritten. Trotz dieser Kritik bleiben gerade die Selbstevaluationsinstrumente die am meisten angewandten Messinstrumente in diesem Bereich. Das liegt m.E. an ihrer Handlichkeit und der Aussagekraft ihrer Ergebnisse: Gesichtspunkte, die die Konstruktion und Verwendung des YOGA-Tests leiteten.

YOGA-Test wurde als Pilotprojekt im Jahre 2000 veröffentlicht. Fantini definiert seine Funktion wie folgt:

„It is designed to help you examine the development of your intercultural communicative competence (ICC, or Intercultural Competence, for short). This pilot document should help you to critically examine your intercultural objectives, serve as guidelines while undergoing an intercultural experience, and provide an assessment tool at various stages of intercultural development" (Fantini 2000, S. 34).

Da sich die Ergebnisse dieses Tests als „normative, formative, as well as summative“ (Fantini 2005, S. 3) erwiesen hatten, wurde er zum Jahr 2005 überarbeitet und im Projekt „Exploring and Assessing Intercultural Competence“ (Juli 2005 - Dezember 2006) eingesetzt. Dieses Projekt wurde im Rahmen der FEIL (Federation of The Experiment in International Living ${ }^{62}$ mit der Unterstützung des „Center for Social Development of the Global Service Institute“ (Washington University in St. Louis, Missouri) erstmals durchgeführt, weitere

${ }_{61}$ Der Begriff „YOGA“ steht für „Your Objectives, Guidelines, and Assessment“

${ }^{62}$ Federation of The Experiment in International Living (FEIL) ist eine der ältesten, weltweit tätigen Organisationen, die im Bereich „interkulturelle Bildung“ agiert. Sie wurde im Jahre 1932 gegründet und vereinigt autonome, nicht kommerziell orientierte Mitgliegsorganisationen in 22 Ländern. FEIL unterstützt eine Reihe von Programmen und Projekten mit dem Ziel Ausbildung, Service und Entwicklung im interkulturellen Bereich. 
Studien sollen folgen. In dieser Pilotphase waren drei Mitgliedsorganisationen von FEIL (Großbritannien, Ecuador und die Schweiz) beteiligt, weitere sind vorgesehen. Die Partizipanten des Projekts sind Menschen mit interkultureller Erfahrung, die sie während eines Auslandsaufenthaltes (Auslandsemester, Auslandseinsatz, Gruppenreise oder Sprachkurs) gesammelt hatten. In der Pilotphase des Projektes wurden sowohl quantitative als auch qualitative Daten gesammelt. Als quantitative Methode wurde der YOGA-Fragebogen eingesetzt, zu den qualitativen zählen die Analyse der Kommentare, Interviews und Anekdoten.

Der YOGA-Test wurde als Grundlage für den Puzzle-Fragebogen gewählt, weil die beiden Instrumente auf ähnlichen theoretischen Modellen basieren. Während Fantinis Modell der interkulturellen Kompetenz aus vier Komponenten (Kenntnisse, Haltungen, Fähigkeiten und Bewusstsein) besteht, enthält das Puzzlemodell drei Komponenten, und zwar Kenntnisse auf der kognitiven Ebene, interkulturelle Sensibilität auf der affektiven Ebene und Fähigkeiten auf der Handlungsebene.

Fantini prüfte die Reliabilität des Messinstruments, indem er unterschiedliche Tests innerhalb des Projektes „Exploring and Assessing Intercultural Competence“ durchführte, die nicht nur der Evaluation der erhobenen Daten, sondern auch der Bewertung des Messinstruments dienten. So wurde das Cronbach Alpha Verfahren angewandt, um die Reliabilität der einzelnen Komponenten zu messen. Im Weiteren wurde die Gewichtung jeder einzelnen Frage innerhalb der vier Komponenten mit Hilfe des Varimax Rotationsverfahrens ausgewertet. Mit dem durchschnittlichen Gewichtungsfaktor $>0.6$ und Crombach Alpha $\geq 0.7$ konnte er die Reliabilität der einzelnen Komponenten feststellen (vgl. Fantini 2005, S. 1720).

Der Schwerpunkt dieser Dissertation liegt auf der Erarbeitung eines Programms zur Entwicklung interkultureller Kompetenzen. Mit dem hier vorgelegten PEIK ist nach Meinung der Verfasserin ein Trainingsprogramm entstanden, das nunmehr zur Erprobung genutzt werden kann. Eine erste Erprobung der Wirksamkeit von PEIK wurde von der Verfasserin durchgeführt, sie ist jedoch nur als Pilotuntersuchung zu verstehen. Mangels eines geeigneteten Instruments wurde ein vorhandenes Messinstrument - dessen Wirksamkeit innerhalb des Projekts „Exploring and Assessing Intercultural Competence“ bereits belegt worden ist - adaptiert. Der von der Verfasserin entwickelte Puzzle-Fragebogen soll die Wirksamkeit des Programms nachweisen, als informelles Verfahren Lernstand und -zuwachs in Bezug auf die detaillierten Unterrichtsziele abbilden. Folglich betrachtet die Auswertung die Beantwortung jeder einzelnen Frage. Eine Evaluierung muss späteren Untersuchungen über- 
lassen bleiben.

Der Puzzle-Test besteht aus einem Einführungsteil und einem Hauptteil. Im Einführungsteil sind einige Informationen über die Person in Form geschlossener Fragen angefordert.

1. Geschlecht:

$\diamond$ weiblich $\quad \diamond$ männlich

2. Waren Sie schon im Ausland?
$\diamond$ ja, weniger als 3 Monate
$\diamond$ ja, mehr als 3 Monate
$\diamond$ nein

3. Haben Sie Freunde aus anderen kulturellen Kreisen?

$\diamond$ ja $\quad \diamond$ nein

Diese Fragen erfassen unabhängige Einflussvariablen für die Analyse der Ergebnisse. Sie erfragen sowohl persönliche Daten als auch kulturelle Erfahrungen der Probanden (Fragen 2 und 3). Die Fragen sind nicht eindeutig zu definieren, sie sind so ausgelegt, dass jeder Proband für sich selbst einzelne Begriffe wie „Ausland“63 oder „Vertreter einer anderen Nation ${ }^{\text {“64 }}$ definieren muss.

Der Hauptteil des Tests besteht aus drei Teilen, entsprechend den drei Komponenten des Puzzle-Modells. Es werden Kenntnisse und Fähigkeiten im interkulturellen Bereich, sowie interkulturelle Sensibilität gemessen.

Um die Entwicklung der interkulturellen Kompetenz im kognitiven Bereich zu messen, wurden weitere 11 Fragen zu kulturübergreifenden Kenntnissen und interkulturellem Bewusstsein gestellt.

1.1. Ich kann den Begriff „Kultur“ definieren und kulturelle Komponenten nennen.

1.2. Ich kann Aspekte nennen, die meine eigene Kultur von der anderen unterscheiden.

1.3. Ich kenne die wichtigsten Normen und Tabus der fremden Kultur, so wie BegrüBungsrituale, Kleidung, Verhaltensregeln etc.

1.4. Ich kann mein eigenes Verhalten und das Verhalten meines fremdkulturellen Ge-

63 Nach dem Zerfall der Sowjetunion existieren in der russischen Sprache zwei Begriffe für das Wort „Ausland“: „näheres Ausland“ und „ferneres Ausland“. Unter dem ersten Begriff sind die ehemaligen Republiken wie Kasachstan, Tatschikistan, Weißrussland etc. zu verstehen. Obwohl der Begriff „,näheres Ausland“ offiziell im Umgang ist, assoziieren die meisten Bürger mit dem Ausdruck „Ausland“ immer noch die Länder außerhalb der ehemaligen Sowjetunion.

64 Unter dem Begriff „Vertreter einer anderen Nation“ können sowohl die Mitbürger aus dem Ausland verstanden werden als auch die nächsten Nachbarvölker wie Barjaten, Jakuten etc. Die Gesellschaft in Irkutsk ist multikulturell, das ist geschichtlich bedingt. Was jeder einzelne Proband darunter versteht, ist ein Zeichen seiner Wahrnehmung, seiner Sensibilität. 
sprächpartners erklären (soziales Verhalten, Zeitorientierungen, Umweltorientierung).

1.5. Ich kann eigene historische und sozialpolitische Tatsachen nennen, die meine eigene Kultur von anderen unterscheiden.

1.6. Ich kann ein Modell der interkulturellen Begegnung und die dabei entstehenden Gefühle und Gedanken beschreiben.

1.7. Es ist mir bewusst, dass es Unterschiede sowohl zwischen den Sprachen als auch zwischen den Kulturen gibt.

1.8. Es ist mir bewusst, dass ich manchmal negativ auf kulturelle Unterschiede reagiere (dabei treten Emotionen wie Angst, Abneigung, Spott, Überlegenheit auf).

1.9. Spezifische Umstände und die Tatsache, dass mein Gesprächspartner zu einer anderen Kultur gehört, wirken auf unsere Kommunikation und verändern sie.

1.10. Mein fremdkultureller Gesprächspartner nimmt mich anders wahr als die Vertreter meiner eigenen Kultur.

1.11. Es ist mir bewusst, dass ich als eine durch die eigene Kultur geprägte Person mit den eigenen Vorlieben und Gewohnheiten agiere.

Zur Messung der Entwicklung der interkulturellen Sensibilität werden den Probanden sieben Aussagen vorgelegt. Sie beziehen sich auf Offenheit und Respekt gegenüber Vertretern von anderen Kulturen; Empathievermögen und Ambiguitätstoleranz sowie die Bereitschaft, die eigenen kulturellen Normen zu relativieren.

2.1. Ich bin zur Kommunikation mit Vertretern der anderen Kultur bereit (ich vermeide sie nicht, ich ziehe den Kreis meiner Landsleute nicht vor).

2.2. Ich interessiere mich für andere Sprachen und Kulturen.

2.3. Ich bemühe mich, bei der Kommunikation mit dem fremdkulturellen Partner mich entsprechend der Normen seiner Kultur zu benehmen.

2.4. Ich versuche die Gründe der negativen Emotionen, die im Laufe der interkulturellen Kommunikation entstehen, zu verstehen und zu lernen, mit ihnen umzugehen.

2.5. Ich interessiere mich für solche Aspekte der anderen Kultur wie Einstellungen, Verhaltensregeln, Geschichte und Traditionen.

2.6. Ich bin bereit, mein eigenes Verhalten entsprechend den Normen der fremden Kultur zu ändern (Sprache, Körpersprache, Verhaltensregeln in verschiedenen Situationen). 
2.7. Ich bin bereit, über die Auswirkungen meines Verhaltens und meiner Worte auf meinen fremdkulturellen Partner nachzudenken.

Um die eigene Einschätzung der Probanden auf der Verhaltensebene zu messen, werden die nächsten acht Aussagen im dritten Teil des Fragebogens gestellt. Sie betreffen die interkulturellen Fähigkeiten der Probanden, und zwar ihre Flexibilität, Kommunikationsfähigkeit, Fähigkeiten zur Selbstreflexion, zur konstruktiven Auseinandersetzung mit dem Fremden und dem Eigenen, zur Vermeidung und zur Klärung von Missverständnissen und zum Umgang mit dem Kulturschock.

3.1. Ich bin flexibel bei der Kommunikation mit dem fremdkulturellen Partner.

3.2. Ich kommuniziere entsprechend den Regeln der fremden Kultur, damit ich meinen fremdkulturellen Partner durch mein Verhalten, meine Kleidung etc. nicht beleidige.

3.3. Ich kann meine eigene und die fremde Kultur miteinander vergleichen.

3.4. Bei der Kommunikation mit den Vertretern einer fremden Kultur benutze ich Strategien zur Verringerung von Kulturschock.

3.5. Ich kann meine Fremdsprachen- und Fremdkulturkenntnisse verbessern.

3.6. Ich benutze eine Vielfalt von Verhaltensstrategien, wenn ich mit Leuten mit einem anderen kulturellen Hintergrund kommuniziere.

3.7. Ich bin fähig, passende Verhaltensstrategien zu finden, wenn bei der interkulturellen Kommunikation etwas falsch gelaufen ist.

3.8. Ich kann aufgrund der sozialpolitischen Faktoren erklären, was meine Kultur von der fremden unterscheidet.

Für die Selbsteinschätzungen der Probanden wurde die Skala des YOGA übernommen. Die Probanden wurden gebeten, jede Frage mit 0 bis 5 zu bewerten: „0“ für keine Kompetenz, „1“ für „,sehr schlecht“, „2“ für „,schlecht“, „3“ für „,befriedigend“, „4“ für „,gut“ und „5“ für ,sehr gut“. Eine weitere Überlegung veranlasste die Verfasserin, diese Bewertungsskala zu übernehmen. Sowohl in russischen Schulen als auch in Hochschulen und Universitäten (darunter auch in dem Ost-Sibirischen Institut des MdI) existiert ein Notensystem von 1 bis 5 für die Bewertung der Lernerfolge der Schüler oder Studierenden durch Lehrer. Dieses Bewertungssystem ist den Probanden vertraut. Alle Fragen wurden von der Verfasserin ins Russische übersetzt und den Probanden auf Russisch vorgelegt. Die Probanden konnten den Fragebogen entweder mit eigenem Namen unterschreiben oder als Codewort den Ge- 
burtstag der Mutter benutzen. Somit konnte der Puzzle-Fragebogen je nach Wunsch der Probanden anonym ausgefüllt werden.

\subsubsection{Datenbearbeitung und statistische Signifikanz}

Nach dem Abschluss des Experiments wurden die aus den Prä- und Posttests erhobenen Daten mit einem statistischen Programm bearbeitet. SPSS Statistik (Statistical Package for the Social Sciences) stellt eine Software dar, die für die Bearbeitung und Analyse von statistischen Daten innerhalb der Sozialwissenschaften geeignet ist. Für die Bearbeitung wurde die Version 18 benutzt.

Für die Analyse der Ergebnisse wurden die Mittelwerte sowohl für jede einzelne Komponente des Puzzle-Modells als auch für das Gesamtergebnis ,interkulturelle Kompetenz“ berechnet. Als einzelne Items wurden die im Puzzle-Test verwendeten Aussagen verwendet. Im Weiteren wurden auch die Differenzen zwischen den Mittelwerten der einzelnen Komponenten und des Gesamtergebnisses ausgerechnet. Als unabhängige Variablen wurden solche wie Gruppenzugehörigkeit, Teilnahme an dem Experiment, Geschlecht, Auslands- und interkulturelle Erfahrungen eingeführt.

Um die Aussagekraft der erhobenen Daten zu überprüfen, wurde die statistische Signifikanz gemessen ${ }^{65}$.

Für die Überprüfung der Signifikanz wurde der T-Test ${ }^{66}$ für gepaarte Stichproben verwendet. Mit diesem Test werden die Ergebnisse einer Stichprobe, die aus zwei Messungen gewonnen worden sind, auf Signifikanz überprüft. Da in der vorliegenden Arbeit mit den Mittelwerten gearbeitet wurde, wurde die Mittelwertdifferenz als statistischer Kennwert für die Überprüfung der Unterschiedshypothesen verwendet, d.h. nach der Analyse der Ergebnisse wurden die Mittelwertdifferenzen zwischen den Werten des Prä- und Posttests sowohl bezüglich der einzelnen Komponenten des Puzzle-Modells als auch des Gesamtergebnisses „,interkulturelle Kompetenz“ in den experimentellen Gruppen auf ihre Signifikanz geprüft. Zum Vergleich wurden auch die Wertedifferenzen in den Kontrollgruppen

65 Der Signifikanztest ermittelt die Wahrscheinlichkeit, mit der das gefundene empirische Ergebnis sowie Ergebnisse, die noch extremer sind als das gefundene Ergebnis, auftreten können, wenn die Populationsverhältnisse der Nullhypothese entsprechen (vgl. Bortz und Döring, 2006, S. 494).

66 Als T-Test wird eine Gruppe von Hypothesentests mit t-verteilter Testprüfgröße bezeichnet, die in der Statistik verwendet werden. Anhand der Mittelwerte wird durch den Test geprüft, ob die Erwartungswerte zweier Grundgesamtheiten einander gleich sind. 
dem T-Test unterzogen.

\section{Analyse der Ergebnisse}

Im folgenden Kapitel werden die Ergebnisse aus den Messungen der interkulturellen Kompetenz im Prä- und Posttest präsentiert und ausgewertet. Im Weiteren werden Unterschiede zwischen Veränderungen der einzelnen Komponenten der interkulturellen Kompetenz verglichen und im Hinblick auf einige unabhängige Variablen wie Teilnahme am Experiment, Geschlecht, Auslandsaufenthalt etc. analysiert und diskutiert.

\subsection{Ergebnisse des Prätests}

Der Prätest wurde in EG1 und EG2 und in der Kontrollgruppe 2151 am 07.09 .04 und in der Kontrollgruppe $2122^{67}$ am 09.09.04 durchgeführt.

Tabelle 3: Mittelwerte für die drei Komponenten der interkulturellen Kompetenz in der Experimental (EG)- und Kontrollgruppe (KG) im Prätest

\begin{tabular}{|l|r|r|r|}
\hline & EG $(\mathrm{n}=30)$ & KG $(\mathrm{n}=23)$ & Gesamt $(\mathrm{n}=53)$ \\
\hline Kenntnisse & 2,9 & 3,07 & 2,98 \\
\hline Sensibilität & 3,14 & 3,3 & 3,21 \\
\hline Fähigkeiten & 3,34 & 3,55 & 3,43 \\
\hline
\end{tabular}

Tabelle 3 enthält die Mittelwerte für die drei Komponenten der interkulturellen Kompetenz für die Experimental- und die Kontrollgruppe zusammengefasst. Die Mittelwerte der Kontrollgruppe liegen etwas höher als die der Experimentalgruppe. Deren Teilnehmer schätzen ihre kulturübergreifenden Kenntnisse im Durchschnitt mit 2,9, ihre interkulturelle Sensibilität mit 3,14 und ihre interkulturellen Fähigkeiten mit 3,34. Nach dem Bewertungssystem, in dem eine „3“ nur „,befriedigend“ bedeutet, schätzen die Teilnehmer ihr interkulturelles Potenzial als relativ gering ein.

$\overline{67}$ Im weiteren Textverlauf werden die Kontrollgruppen 2151 und 2122 KG1 und KG2 genannt. 
Tabelle 4: Mittelwerte für die einzelnen Aussagen zur Komponente „Kenntnisse“ in der Experimental- (EG) und Kontrollgruppe (KG) im Prätest

\begin{tabular}{|c|c|c|}
\hline Aussagen & $\mathrm{EG}$ & $\mathrm{KG}$ \\
\hline $\begin{array}{l}\text { 1.1. Ich kann den Begriff „Kultur" definieren und kulturelle Komponenten nen- } \\
\text { nen. }\end{array}$ & 2,7 & 2,78 \\
\hline $\begin{array}{l}\text { 1.2. Ich kann Aspekte nennen, die meine eigene Kultur von der anderen un- } \\
\text { terscheiden. }\end{array}$ & 3,33 & 3,73 \\
\hline $\begin{array}{l}\text { 1.3. Ich kenne die wichtigsten Normen und Tabus der fremden Kultur, so wie } \\
\text { Begrüßungsrituale, Kleidung, Verhaltensregeln etc. }\end{array}$ & 2,47 & 2,78 \\
\hline $\begin{array}{l}\text { 1.4. Ich kann mein eigenes Verhalten und das Verhalten meines fremdkultu- } \\
\text { rellen Gesprächspartners erklären (soziales Verhalten, Zeitorientierun- } \\
\text { gen, Umweltorientierung) }\end{array}$ & 3,13 & 3,22 \\
\hline $\begin{array}{l}\text { 1.5. Ich kann einige historische und sozialpolitische Tatsachen nennen, die } \\
\text { meine eigene Kultur von einer anderen unterscheiden. }\end{array}$ & 3,5 & 3,57 \\
\hline $\begin{array}{l}\text { 1.6. Ich kann ein Modell der interkulturellen Begegnung und die dabei entste- } \\
\text { henden Gefühle und Gedanken beschreiben. }\end{array}$ & 2,83 & 2,65 \\
\hline $\begin{array}{l}\text { 1.7. Es ist mir bewusst, dass es Unterschiede zwischen den Sprachen und } \\
\text { den Kulturen gibt. }\end{array}$ & 4,3 & 4,83 \\
\hline $\begin{array}{l}\text { 1.8. Es ist mir bewusst, dass ich manchmal negativ auf kulturelle Unterschie- } \\
\text { de reagiere (dabei treten Emotionen wie Angst, Abneigung, Spott, Über- } \\
\text { legenheit etc. auf) }\end{array}$ & 1,67 & 1,91 \\
\hline $\begin{array}{l}\text { 1.9. Spezifische Umstände und die Tatsache, dass mein Gesprächspartner } \\
\text { zu einer anderen Kultur gehört, wirken auf unsere Kommunikation und } \\
\text { verändern sie. }\end{array}$ & 1,7 & 1,83 \\
\hline $\begin{array}{l}\text { 1.10. Mein fremdkultureller Gesprächspartner nimmt mich anders wahr als } \\
\text { die Vertreter seiner eigenen Kultur. }\end{array}$ & 2,4 & 2,3 \\
\hline $\begin{array}{l}\text { 1.11. Es ist mir bewusst, dass ich als eine durch die eigene Kultur geprägte } \\
\text { Person mit eigenen Vorlieben und Gewohnheiten agiere. }\end{array}$ & 3,9 & 4,17 \\
\hline
\end{tabular}

In Tabelle 4 sind die Mittelwerte für die einzelnen Aussagen der fachübergreifenden Kenntnisse, der Experimental- und der Kontrollgruppe angegeben.

Auch hier liegen die Werte der Kontrollgruppe für einige Aussagen höher als die der Experimentalgruppe. Das betrifft z.B. die Fragen 1.2 und 1.7. Bei anderen Aussagen liegen die beiden Gruppen mit einiger Relevanz auf gleichem Niveau. Bei verschiedenen Aussagen sind die Differenzen groß, z.B. bei der Aussage 1.7. Die Teilnehmer der beiden Gruppen haben sich relativ hoch bewertet: mit Mittelwert „4,3“ in den EG und Mittelwert „4,8“ in den KG.; auch bezüglich ihrer kulturellen Selbstreflexion in Aussage 1.11., und zwar mit 3,9 in den EG und 4,2 in den KG. Im Gegensatz dazu liegen die Selbstbewertungen der Teilnehmer zu den Aussagen 1.8. und 1.9. sehr niedrig. Das heißt, während sie sich bei der Selbstwahrnehmung relativ hoch bewertet haben, konnten sie die Auswirkungen der Zuge- 
hörigkeit zu einer anderen Gruppe auf die interkulturelle Kommunikation nicht bewusst wahrnehmen: So lassen sich die Werte zu Aussagen 1.9. und 1.8. interpretieren. Negative Emotionen bei einem interkulturellen Kontakt wahrzunehmen und zu reflektieren, ist bekanntlich eine der schwersten Aufgaben sowohl bei der Ausfüllung dieses Fragebogens als auch im realen Leben. Das ist m.E. durch zwei Tatsachen zu erklären: Zum einen ist die Natur des interkulturellen Bewusstseins relativ kompliziert, zum anderen sind solche Einstellungen durch die in der Sowjetunion propagierte Haltung wie Gleichberechtigung der Völker zu erklären, die den Teilnehmern durch die Erziehung ihrer Eltern vermittelt wurden. Negative Emotionen gegenüber Vertretern anderer Nationen zu hegen, wurde als Folge schlechter Erziehung bewertet.

Was die anderen Aussagen betrifft, liegen die Mittelwerte zwischen 2 und 4. Das ist ein relativ niedriger Index auf der Bewertungsskala.

Tabelle 5: Mittelwerte für die einzelnen Aussagen zur Komponente „Sensibilität“ in der Experimental- (EG) und Kontrollgruppe (KG) im Prätest

\begin{tabular}{|l|r|r|}
\hline Aussagen & EG & KG \\
\hline $\begin{array}{l}\text { 2.1. Ich bin zur Kommunikation mit Vertretern der anderen Kultur bereit (ich } \\
\text { vermeide sie nicht, ich ziehe den Kreis meiner Landsleute nicht vor). }\end{array}$ & 3,73 & 4,00 \\
\hline $\begin{array}{l}\text { 2.1. Ich interessiere mich für andere Sprachen und Kulturen. } \\
\text { len Partner entsprechend den Normen seiner Kultur zu benehmen. }\end{array}$ & 3,37 & 3,61 \\
\hline $\begin{array}{l}\text { 2.3. Ich bemühe mich, mich bei der Kommunikation mit dem fremdkulturel- } \\
\text { terkulturellen Kommunikation entstehen, zu verstehen und zu lernen, } \\
\text { mit innen umzugehen. }\end{array}$ & 2,83 \\
\hline $\begin{array}{l}\text { 2.5. Ich interessiere mich für solche Aspekte der anderen Kultur wie Einstel- } \\
\text { lungen, Verhaltensregeln, Geschichte und Traditionen. }\end{array}$ & 3,83 \\
\hline $\begin{array}{l}\text { 2.6. Ich bin bereit, mein eigenes Verhalten entsprechend den Normen der } \\
\text { fremden Kultur zu ändern (Sprache, Körpersprache, Verhaltensregeln in } \\
\text { verschiedenen Situationen). }\end{array}$ & 3,33 \\
\hline $\begin{array}{l}\text { 2.7. Ich bin bereit, über die Auswirkungen meines Verhaltens und meiner } \\
\text { Worte auf meinen fremdkulturellen Partner nachzudenken. }\end{array}$ & 2,43 \\
\hline
\end{tabular}

In der Tabelle 5 sind die Ergebnisse des Prätests bezogen auf die affektive Seite der interkulturellen Kompetenz, und zwar interkulturelle Sensibilität, präsentiert. Unter der interkulturellen Sensibilität wird hier ein Komplex aus unterschiedlichen Gefühlen, Einstellungen und Fähigkeiten verstanden, die den Menschen dazu befähigen, die Besonderheiten sowohl des Denkens, Fühlens und Handels des fremdkulturellen Partners als auch der kom- 
munikativen Situation selbst wahrzunehmen und zu akzeptieren und davon abhängig sein Verhalten und seine Gefühle zu steuern. Die interkulturelle Sensibilität schließt folgende Komponenten ein: Empathie; Bereitschaft, die eigenen kulturellen Normen zu relativieren; Offenheit und Respekt; Ambiguitätstoleranz.

Die Mittelwerte für die ersten drei Aussagen sind für die Kontrollgruppen höher als für die EG, bei den restlichen sind die Werte relativ gleich. Offenheit und Interesse gegenüber Vertretern der anderen Kulturen zeigten die Teilnehmer bei der Selbsteinschätzung zu den Aussagen 2.1. und 2.2.. Die Mittelwerte liegen im Bereich 3,4 bis 4 Punkte. Das eigene Emphatievermögen wurde durchschnittlich mit 3,5 bewertet; dabei wiesen die beiden Probandengruppen die größten Unterschiede auf. Das beweisen die Werte zur Aussage 2.3.. Am schwächsten ist die Bereitschaft, die eigenen kulturellen Normen zu relativieren, bei den Probanden ausgeprägt. Die Mittelwerte zur Aussage 2.6. sind mit 2,4 in den EG und mit 2,5 in den KG die niedrigsten, was die Sensibilität angeht.

Tabelle 6: Mittelwerte für die einzelnen Aussagen zur Komponente „Fähigkeiten“ in der Experimental- (EG) und Kontrollgruppe (KG) im Prätest

\begin{tabular}{|l|r|r|}
\hline Aussagen & EG & KG \\
\hline $\begin{array}{l}\text { 3.1. Ich bin flexibel bei der Kommunikation mit dem fremdkulturellen Partner. } \\
\text { ich meinen fremdkulturellen Partner durch mein Verhalten, meine Klei- } \\
\text { dung etc. nicht beleidige. }\end{array}$ & 2,87 & 3,17 \\
\hline $\begin{array}{l}\text { 3.2. Ich kommuniziere entsprechend den Regeln der fremden Kultur, damit } \\
\text { 3.3. Ich kann meine eigene und die fremde Kultur miteinander vergleichen. }\end{array}$ & 4,13 \\
\hline $\begin{array}{l}\text { 3.4. Bei der Kommunikation mit den Vertretern einer fremden Kultur benutze } \\
\text { ich Strategien zur Verringerung von Kulturschok. }\end{array}$ & 2,50 \\
\hline $\begin{array}{l}\text { 3.5. Ich kann meine Fremdsprachen- und Fremdkulturkenntnisse verbes- } \\
\text { sern. }\end{array}$ & 3,80 \\
\hline $\begin{array}{l}\text { 3.6. Ich benutze eine Vielfalt von Verhaltensstrategien, wenn ich mit Leuten } \\
\text { mit einem anderen kulturellen Hintergrund kommuniziere. }\end{array}$ & 2,48 \\
\hline $\begin{array}{l}\text { 3.7. Ich bin fähig, passende Verhaltensstrategien zu finden, wenn bei der in- } \\
\text { terkulturellen Kommunikation etwas falsch gelaufen ist. }\end{array}$ & 4,04 \\
\hline $\begin{array}{l}\text { 3.8. Ich kann aufgrund der sozialpolitischen Faktoren erklären, was meine } \\
\text { Kultur von der fremden unterscheidet. }\end{array}$ & 3,97 \\
\hline
\end{tabular}

In Tabelle 6 sind die Ergebnisse des Prätests bezüglich der verhaltensbezogenen Komponenten der interkulturellen Kompetenz, und zwar der interkulturellen Fähigkeiten, dargestellt. Diese Komponente schließt die Fähigkeiten des Lernenden ein, mit Hilfe seiner Kenntnisse und seiner interkulturellen Sensibilität adäquat und erfolgreich in Situationen 
interkultureller Kontakte zu kommunizieren.

Alle Mittelwerte liegen im Bereich zwischen 2,5 und 4,3, und genauso wie bei den ersten Komponenten schneiden die Kontrollgruppen im Durchschnitt besser als die experimentellen Gruppen ab. Am niedrigsten waren die Ergebnisse zu den Aussagen 3.4. und 3.6. Dabei geht es in beiden Fällen um das Vorhandensein von Strategien, im ersten Fall zur Verringerung des Kulturschocks und im zweiten um die Verhaltensstrategien zur Vermeidung von Missverständnissen. Die niedrigere Selbsteinschätzung der Teilnehmer in den beiden Aussagen ist m.E. zum Teil durch die Benutzung relativ schwieriger Begriffe wie „Verhaltensstrategien“ und „kultureller Stress“ in den Fragen zu erklären. Während der Begriff „Verhaltensstrategie" noch aus dem Unterricht in der Psychologie bekannt ist, ist die Redewendung „,kultureller Stress“ für einen Teil der Teilnehmer neu.

Die Selbsteinschätzungswerte für die Entwicklung der interkulturellen Kommunikationsfähigkeit streuen breit. Bei der Aussage 3.2., die neben der Kommunikationsfähigkeit auch nach der Fähigkeit zur Selbstreflexion fragt, liegen die Mittelwerte wie folgt: 3,1 in den EG und 4 in den KG. Die Frage 3.1., die außer auf Kommunikationsfähigkeit auch auf Flexibilität abzielt, wurde mit 2,9 in den EG und mit 3,2 in den KG beantwortet.

Die Bewertung der Fähigkeit zur konstruktiven Auseinandersetzung mit dem Eigenen und dem Fremden ist relativ hoch. Bei der Aussage 3.3. haben sich die Teilnehmer im Durchschnitt mit 4,3 in den EG und 3,8 in den KG bewertet. Diese relativ hohe Selbsteinschätzung bestätigen auch die Ergebnisse zur Aussage 3.8. mit Mittelwerten von 3,7 (EG) und 3,9 (KG). Relativ hoch wurde auch die Fähigkeit zur Klärung von Missverständnissen eingeschätzt. Zur Aussage 3.7. haben sich die Teilnehmer im Durchschnitt mit 4 (EG) und 4,3 (KG) bewertet.

\subsection{Ergebnisse des Posttests}

In Tabelle 7 sind die Ergebnisse des Posttests zusammengefasst. Hier sind Werte für die drei Komponenten Interkultureller Kompetenz für KG und EG angegeben.

Tabelle 7: Mittelwerte für die drei Komponenten der interkulturellen Kompetenz in der Experimental- (EG) und Kontrollgruppe (KG) im Posttest

\begin{tabular}{|l|r|r|r|}
\hline & EG $(\mathrm{n}=30)$ & KG $(\mathrm{n}=23)$ & Gesamt $(\mathrm{n}=53)$ \\
\hline Kenntnisse & 3,49 & 2,89 & 3,23 \\
\hline Sensibilität & 3,66 & 3,34 & 3,52 \\
\hline Fähigkeiten & 3,93 & 3,57 & 3,78 \\
\hline
\end{tabular}


Im Unterschied zu den Ergebnissen des Prätests, liegen die Mittelwerte der experimentellen Gruppen im Posttest deutlich höher als in den Kontrollgruppen, außerdem liegen alle Werte im Bereich 3,5 und höher. Dabei schätzen die Teilnehmer ihre interkulturellen Fähigkeiten als besser entwickelt ein als ihre Kenntnisse oder interkulturelle Sensibilität. In den folgenden Tabellen werden die Ergebnisse für jede einzelne Komponente der interkulturellen Kompetenz präsentiert und anschließend diskutiert. In Tabelle 8 wird die kognitive Seite der Kompetenz, kulturübergreifende Kenntnisse und damit zusammenhängendes Bewusstsein präsentiert. Es folgt der Vergleich der Mittelwerte für die experimentelle und für die Kontrollgruppen für jede der 13 Aussagen:

Tabelle 8: Mittelwerte für die einzelnen Aussagen zur Komponente „Kenntnisse“ in der Experimental- (EG) und Kontrollgruppe (KG) im Posttest

\begin{tabular}{|c|c|c|}
\hline Aussagen & $\mathrm{EG}$ & $\mathrm{KG}$ \\
\hline $\begin{array}{l}\text { 1.1. Ich kann den Begriff „Kultur“ definieren und kulturelle Komponenten nen- } \\
\text { nen. }\end{array}$ & 3,63 & 3,05 \\
\hline $\begin{array}{l}\text { 1.2. Ich kann Aspekte nennen, die meine eigene Kultur von der anderen un- } \\
\text { terscheiden. }\end{array}$ & 3,97 & 3,82 \\
\hline $\begin{array}{l}\text { 1.3. Ich kenne die wichtigsten Normen und Tabus der fremden Kultur, so wie } \\
\text { Begrüßungsrituale, Kleidung, Verhaltensregeln etc. }\end{array}$ & 3,70 & 3,23 \\
\hline $\begin{array}{l}\text { 1.4. Ich kann mein eigenes Verhalten und das Verhalten meines fremdkultu- } \\
\text { rellen Gesprächspartners erklären (soziales Verhalten, Zeitorientierun- } \\
\text { gen, Umweltorientierung) }\end{array}$ & 3,77 & 3,32 \\
\hline $\begin{array}{l}\text { 1.5. Ich kann einige historische und sozialpolitische Tatsachen nennen, die } \\
\text { meine eigene Kultur von einer anderen unterscheiden. }\end{array}$ & 3,67 & 3,86 \\
\hline $\begin{array}{l}\text { 1.6. Ich kann ein Modell der interkulturellen Begegnung und die dabei entste- } \\
\text { henden Gefühle und Gedanken beschreiben. }\end{array}$ & 3,41 & 2,86 \\
\hline $\begin{array}{l}\text { 1.7. Es ist mir bewusst, dass es Unterschiede zwischen den Sprachen und } \\
\text { den Kulturen gibt. }\end{array}$ & 4,88 & 4,48 \\
\hline $\begin{array}{l}\text { 1.8. Es ist mir bewusst, dass ich manchmal negativ auf kulturelle Unterschie- } \\
\text { de reagiere (dabei treten Emotionen wie Angst, Abneigung, Spott, Über- } \\
\text { legenheit etc. auf) }\end{array}$ & 2,10 & 1,13 \\
\hline $\begin{array}{l}\text { 1.9. Spezifische Umstände und die Tatsache, dass mein Gesprächspartner } \\
\text { zu einer anderen Kultur gehört, wirken auf unsere Kommunikation und } \\
\text { verändern sie. }\end{array}$ & 2,60 & 1,35 \\
\hline $\begin{array}{l}\text { 1.10. Mein fremdkultureller Gesprächspartner nimmt mich anders wahr als } \\
\text { die Vertreter seiner eigenen Kultur. }\end{array}$ & 3,33 & 1,61 \\
\hline $\begin{array}{l}\text { 1.11. Es ist mir bewusst, dass ich als eine durch die eigene Kultur geprägte } \\
\text { Person mit eigenen Vorlieben und Gewohnheiten agiere. }\end{array}$ & 4,07 & 4,04 \\
\hline
\end{tabular}


Bei der Aussage über den Begriff „Kultur“ (Frage 1.1.) und über die kulturelle Komponente (Aussage 1.2.) haben sich die Teilnehmer der EG relativ hoch eingeschätzt. Im Vergleich zur KG $(3,0)$ liegt der Mittelwert für die Aussage 1.1. bei 3,6. Etwas höher liegt sogar der Mittelwert für die Aussage 1.2.. Mit fast 4 Punkten überragt er das relativ gute Ergebnis der Kontrollgruppe $(3,8)$.

Auch bei der Einschätzung des Bewusstseins betreffend die Andersartigkeit schneiden die Teilnehmer der experimentellen Gruppen relativ hoch ab. Das bestätigen die Werte zur Aussage 1.7.. Auf den ersten Blick liegen die Werte überdurchschnittlich hoch, und mit fast 4.9 Punkten ist es der höchste Wert im Bereich „Kenntnisse“. Etwas niedriger, aber immer noch mit hohen Werten schneidet die Aussage 1.4. ab: 3,8 ist der Durchschnittswert der EG und 3,3 der der KG.

Dabei fällt es den Teilnehmern schwer, die Auswirkungen dieser Andersartigkeit des fremdkulturellen Partners auf die interkulturelle Kommunikation zu akzeptieren. Die Durchschnittswerte bei der Aussage 1.9. liegen auf relativ niedrigem Niveau: 2,6 in den EG und 1,3 in den $\mathrm{KG}$.

Ein ähnliches Bild ist bei den Kenntnissen und der Bewusstheit für die eigene kulturelle Prägung zu beobachten. Obwohl die Teilnehmer die eigene kulturelle Prägung bewusst wahrnehmen (Aussage 1.11.: 4 EG und $4 \mathrm{KG}$ ) und auch Kenntnisse über den eigenen kulturellen Hintergrund haben (Aussage 1.5.: 3,7 EG und 3,9 KG), sind ihre Auswirkungen auf die Kommunikation nicht ganz klar. Das ist bei den Werten zu den Aussagen 1.10. und 1.8. zu sehen. Bei der Aussage 1.10. weist aber der Wert in den EG $(3,3)$ einen großen Abstand zu dem Wert der KG $(1,6)$ auf. Die eigenen, besonders die negativen Emotionen zu reflektieren, fällt den Teilnehmern am schwersten. Die Werte zu Aussage 1.8. sind immer sehr niedrig: 2,1 in den EG und 1,1 in den KG.

Die affektive Komponente der interkulturellen Kompetenz ist mit den postexperimentellen Werten in der Tabelle 9 präsentiert:

Tabelle 9: Mittelwerte für die einzelnen Aussagen zur Komponente „Sensibilität“ in der Experimental- (EG) und Kontrollgruppe (KG) im Posttest

\begin{tabular}{|l|r|r|}
\hline Aussagen & EG & \multicolumn{2}{|l|}{ KG } \\
\hline $\begin{array}{l}\text { 2.1. Ich bin zur Kommunikation mit Vertretern der anderen Kultur bereit (ich } \\
\text { vermeide sie nicht, ich ziehe den Kreis meiner Landsleute nicht vor). }\end{array}$ & 4,17 & 3,91 \\
\hline 2.2. Ich interessiere mich für andere Sprachen und Kulturen. & 4,03 & 3,39 \\
\hline $\begin{array}{c}\text { 2.3. Ich bemühe mich, mich bei der Kommunikation mit dem fremdkulturel- } \\
\text { len Partner entsprechend der Normen seiner Kultur zu benehmen. }\end{array}$ & 4,00 & 3,65 \\
\hline
\end{tabular}




\begin{tabular}{|l|r|r|}
\hline $\begin{array}{l}\text { 2.4. Ich versuche die Gründe der negativen Emotionen, die im Laufe der in- } \\
\text { terkulturellen Kommunikation entstehen, zu verstehen und zu lernen, } \\
\text { mit ihnen umzugehen. }\end{array}$ & 2,93 \\
\hline $\begin{array}{l}\text { 2.5. Ich interessiere mich für solche Aspekte der anderen Kultur wie Einstel- } \\
\text { lungen, Verhaltensregeln, Geschichte und Traditionen. }\end{array}$ & 3,67 \\
\hline $\begin{array}{l}\text { 2.6. Ich bin bereit, mein eigenes Verhalten entsprechend den Normen der } \\
\text { fremden Kultur zu ändern (Sprache, Körpersprache, Verhaltensregeln in } \\
\text { verschiedenen Situationen). }\end{array}$ & 3,13 \\
\hline $\begin{array}{l}\text { 2.7. Ich bin bereit, über die Auswirkungen meines Verhaltens und meiner } \\
\text { Worte auf meinen fremdkulturellen Partner nachzudenken. }\end{array}$ & 3,67 \\
\hline
\end{tabular}

Beim ersten Blick fällt auf, dass die Werte der EG relativ hoch sind und bei den drei ersten Aussagen sogar über 4 Punkten liegen. D.h. bei den Aussagen über Interesse und Offenheit gegenüber anderen Kulturen gaben sich die Teilnehmer recht gute Noten. So z.B. liegt der Mittelwert für die Aussage 2.1. für die EG bei 4,2 und die KG bei 3,9, für Aussage 2.2.: für die EG bei 4. Wenn es in Frage darauf ankommt zu beantworten, auf welche Aspekte der fremden Kultur sich dieses Interesse bezieht, relativiert sich der Mittelwert um einige Punkte. Aussage 2.5. wurde in den EG mit 3,7 und in den KG mit 3,5 bewertet. Die Ergebnisse in der EG liegen immer noch höher als die der KG, besonders groß ist der Unterschied in den Mittelwerten zu Aussage 2.2..

Relativ hoch bewerten sich die Teilnehmer bei der Aussage über Empathie. Sie zeigen sich bereit, die Perspektive des fremdkulturellen Partners im Gespräch anzunehmen. (Aussage 2.3.: 4 (EG) und 3,6 (KG). Dass dies aber mit Verzicht auf die eigene kulturelle Position verbunden ist, z.B. auf die als „normal““ empfundenen Verhaltensregeln oder Körpersprache, ist den Teilnehmern weniger klar (Aussage 2.6.: 3,1 (EG) und 3,3 (KG). Bei der Bewertung dieser Aussage haben die Teilnehmer der EG sogar schlechter abgeschnitten als die Teilnehmer der KG.

Genauso niedrig wie die Bereitschaft, die eigenen kulturellen Normen zu relativieren, bewerten die Teilnehmer die eigene Bereitschaft, mit negativen Emotionen umzugehen. $\mathrm{Zu}$ den Angaben zur Aussage 2.4. liegt der Mittelwert der EG bei 2,9 und damit immer noch wesentlich höher als der der KG mit 2,2. 
Tabelle 10: Mittelwerte für die einzelnen Aussagen zur Komponente „Fähigkeiten“ in der Experimental- (EG) und Kontrollgruppe (KG) im Posttest

\begin{tabular}{|l|r|r|}
\hline Aussagen & EG & KG \\
\hline $\begin{array}{l}\text { 3.1. Ich bin flexibel bei der Kommunikation mit dem fremdkulturellen Partner. } \\
\text { ich meinen fremdkulturellen Partner durch mein Verhalten, meine Klei- } \\
\text { dung etc. nicht beleidige. }\end{array}$ & 3,67 & 3,17 \\
\hline $\begin{array}{l}\text { 3.2. Ich kommuniziere entsprechend den Regeln der fremden Kultur, damit } \\
\text { 3.3. Ich kann meine eigene und die fremde Kultur miteinander vergleichen. }\end{array}$ & 3,90 \\
\hline $\begin{array}{l}\text { 3.4. Bei der Kommunikation mit Vertretern einer fremden Kultur benutze ich } \\
\text { Strategien zur Verringerung von Kulturschock. }\end{array}$ & 2,83 \\
\hline $\begin{array}{l}\text { 3.5. Ich kann meine Fremdsprachen- und Fremdkulturkenntnisse verbes- } \\
\text { sern. }\end{array}$ & 4,23 \\
\hline $\begin{array}{l}\text { 3.6. Ich benutze eine Vielfalt von Verhaltensstrategien, wenn ich mit Leuten } \\
\text { mit einem anderen kulturellen Hintergrund kommuniziere. }\end{array}$ & 3,09 \\
\hline $\begin{array}{l}\text { 3.7. Ich bin fähig, passende Verhaltensstrategien zu finden, wenn bei der in- } \\
\text { terkulturellen Kommunikation etwas falsch gelaufen ist. }\end{array}$ & 3,63 \\
\hline $\begin{array}{l}\text { 3.8. Ich kann aufgrund der sozialpolitischen Faktoren erklären, was meine } \\
\text { Kultur von der fremden unterscheidet. }\end{array}$ & 4,60 \\
\hline
\end{tabular}

Am besten haben die Teilnehmer der EG ihre Fähigkeit zur konstruktiven Auseinandersetzung mit dem Eigenen und dem Fremden bewertet. Bei dieser Fähigkeit geht es darum, in unterschiedlichen Sachverhalten die beiden Pole „Fremdes“ und „Eigenes“ wahrzunehmen, zu reflektieren, miteinander in Verbindung zu bringen, zu analysieren, um weiter entsprechende Verhaltensstrategien auswählen zu können. Mit 4,5 in den EG und mit 4 in den KG haben die Teilnehmer ihre Fähigkeit, die zwei Kulturen (eigene und fremde) zu vergleichen, bewertet (Aussage 3.3.). Die Fähigkeit, die beiden Kulturen in Verbindung zu setzen und zu analysieren scheint in der durchschnittlichen Bewertung mit 4,1 (EG) und 3,6 (KG) auch gut entwickelt zu sein (Aussage 3.8.).

Aus Sicht der Teilnehmer beherrschen sie Strategien zur Klärung von Missverständnissen besser als Strategien zu ihrer Vermeidung. Das beweist das Vergleich der Ergebnisse zu den Fragen 3.6. und 3.7.. Während die Mittelwerte zu der Frage 3.7. sehr hoch sind, und zwar 4,6 (EG) und 4,2 (KG), schneiden die Teilnehmer bei der Frage 3.6. eher mittelmäßig $\mathrm{ab}$ (3,6 für die EG und 3,3 für die $\mathrm{KG})$.

Auch die Aussage 3.1. über die Flexibilität in der interkulturellen Kommunikation wurde von den Teilnehmern der EG auf dem gleichen Niveau bewertet. Die Ergebnisse der EG $(3,7)$ liegen eindeutig über den Ergebnissen der KG $(3,2)$. Auch die kommunikativen Kom- 
petenzen im Bereich des interkulturellen Diskurses können als ,gut entwickelt“ bewertet werden, das belegen die Ergebnisse zur Aussage 3.2.: 3,9 (EG) und 3,7 (KG).

Die Ergebnisse zu der Aussage 3.4. fallen aus dem gesamten Bild heraus. Mit 2,9 liegen die Teilnehmer der EG auf dem gleichen Niveau wie die Teilnehmer der KG, und dieses Niveau ist relativ niedrig. Es ist damit zu erklären, dass auf den Begriff „kultureller Stress“ im PEIK nicht eingegangen wurde, und die Teilnehmer der EG keinerlei Möglichkeiten hatten, ihre Fähigkeiten in diesem Bereich zu entwickeln.

\subsection{Veränderungen der interkulturellen Kompetenz entsprechend der drei Komponenten des Puzzle-Modells}

Die Entwicklung der interkulturellen Kompetenz ist ein komplexer und langfristiger Vorgang. Innerhalb des oben vorgestellten Experiments und mithilfe des PEIK wurde versucht, im Rahmen des Fremdsprachenunterrichts während eines begrenzten Zeitraums gezielt daran zu arbeiten. Als theoretische Grundlage wurde das Puzzle-Modell der interkulturellen Kompetenz benutzt, die aus drei Komponenten: Kenntnissen, Fähigkeiten und interkultureller Sensibilität besteht. Entsprechend dieser Komponenten wurde auch das Niveau der Kompetenzentwicklung der Teilnehmer vor und nach dem Experiment gemessen. In diesem Kapitel wird festgestellt, ob das von der Verfasserin entwickelte Programm tatsächlich Auswirkungen auf die Entwicklung der interkulturellen Kompetenz der Teilnehmer hat. Zum Vergleich wurden Prä- und Posttest auch in der KG durchgeführt, deren Teilnehmer an dem oben erwähnten Experiment nicht teilgenommen haben. Im vorliegenden Kapitel werden die Ergebnisse der beiden Tests verglichen. Die zu überprüfende Annahme ist, dass der Lernzuwachs der EG-Teilnehmer größer ist als der der KG, und zwar in allen Komponenten des Puzzle-Modells. Anschließend werden Veränderungen diskutiert und entsprechende Schlussfolgerungen gezogen.

\subsubsection{Veränderungen der Komponente „Kenntnisse“6}

Die kognitive Komponente des Puzzle-Modells umfasst kulturübergreifende Kenntnisse und interkulturelles Bewusstsein. Die Vermittlung interkultureller Kenntnisse ist seit langem ein anerkanntes Teilziel des Sprachunterrichts Der Lernzuwachs in diesem Bereich ist 
relativ leicht nachvollziehbar und auch für die Teilnehmer selbst zu bemerken und im Posttest $\mathrm{zu}$ benennen. Unter der Bedingung, dass die Inhalte des Programms wirksam sind, werden gerade in diesem Bereich die deutlichsten Veränderungen erwartet.

In der folgenden Abbildung sind die Veränderungen im Bereich „Kenntnisse/Bewusstsein“ dargestellt. Das Niveau der Kenntnisse vor dem Experiment ist in beiden Gruppen nahezu gleich mit einem geringfügig höheren Durchschnittswert der KG mit 3.07, bei 2,9 der EG. Bei der experimentellen Gruppen sind deutliche Veränderungen zu sehen: Der Mittelwert steigt um 0,65 auf 3,56 Punkte, dagegen sinkt der Wert der KG geringfügig. Das bestätigt die Annahme, dass die Inhalte des PEIK positive Veränderungen im Kenntnisstand der Teilnehmer bewirken können.

Abbildung 7: Prä-/Posttest-Vergleich zur Komponente „Kenntnisse“: EG versus KG

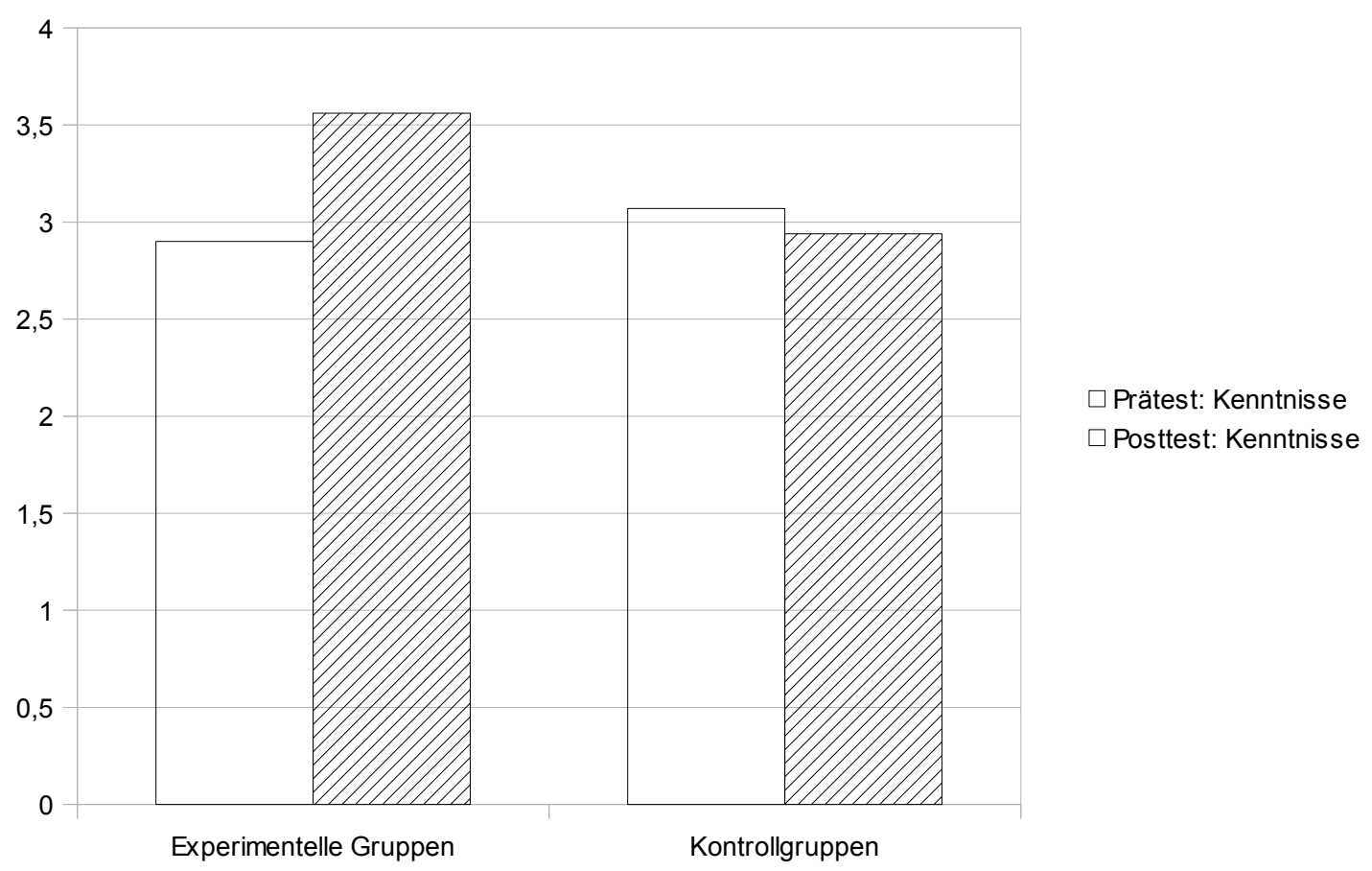

Im Weiteren wird untersucht, welche Einzelaspekte der Komponente „Kenntnisse“ sich verändert haben. 
Tabelle 11: Mittelwerte für die einzelnen Aussagen zur Komponente „Kenntnisse“ in der Experimentalgruppe

\begin{tabular}{|c|c|c|c|}
\hline Aussagen & Prätest & Posttest & Differenz \\
\hline $\begin{array}{l}\text { 1.1. Ich kann den Begriff „Kultur“ definieren und kulturelle Kom- } \\
\text { ponenten nennen. }\end{array}$ & 2,70 & 3,63 & 0,93 \\
\hline $\begin{array}{l}\text { 1.2. Ich kann Aspekte nennen, die meine eigene Kultur von der } \\
\text { anderen unterscheiden. }\end{array}$ & 3,33 & 3,97 & 0,63 \\
\hline $\begin{array}{l}\text { 1.3. Ich kenne die wichtigsten Normen und Tabus der fremden } \\
\text { Kultur, so wie Begrüßungsrituale, Kleidung, Verhaltensregeln } \\
\text { etc. }\end{array}$ & 2,47 & 3,70 & 1,23 \\
\hline $\begin{array}{l}\text { 1.4. Ich kann mein eigenes Verhalten und das Verhalten meines } \\
\text { fremdkulturellen Gesprächspartners erklären (soziales Ver- } \\
\text { halten, Zeitorientierungen, Umweltorientierung). }\end{array}$ & 3,13 & 3,77 & 0,63 \\
\hline $\begin{array}{l}\text { 1.5. Ich kann einige historische und sozialpolitische Tatsachen } \\
\text { nennen, die meine eigene Kultur von einer anderen unter- } \\
\text { scheiden. }\end{array}$ & 3,50 & 3,67 & 0,17 \\
\hline $\begin{array}{l}\text { 1.6. Ich kann ein Modell der interkulturellen Begegnung und die } \\
\text { dabei entstehenden Gefühle und Gedanken beschreiben. }\end{array}$ & 2,83 & 3,41 & 0,59 \\
\hline $\begin{array}{l}\text { 1.7. Es ist mir bewusst, dass es Unterschiede zwischen den } \\
\text { Sprachen und den Kulturen gibt. }\end{array}$ & 4,30 & 4,87 & 0,57 \\
\hline $\begin{array}{l}\text { 1.8. Es ist mir bewusst, dass ich manchmal negativ auf kulturelle } \\
\text { Unterschiede reagiere (dabei treten Emotionen wie Angst, } \\
\text { Abneigung, Spott, Überlegenheit etc. auf). }\end{array}$ & 1,67 & 2,10 & 0,43 \\
\hline $\begin{array}{l}\text { 1.9. Spezifische Umstände und die Tatsache, dass mein Ge- } \\
\text { sprächspartner zu einer anderen Kultur gehört, wirken auf } \\
\text { unsere Kommunikation und verändern sie. }\end{array}$ & 1,70 & 2,6 & 0,90 \\
\hline $\begin{array}{l}\text { 1.10.Mein fremdkultureller Gesprächspartner nimmt mich anders } \\
\text { wahr als die Vertreter seiner eigenen Kultur. }\end{array}$ & 2,40 & 3,33 & 0,93 \\
\hline $\begin{array}{l}\text { 1.11. Es ist mit bewusst, dass ich als eine durch die eigene Kul- } \\
\text { tur geprägte Person mit den eigenen Vorlieben und Ge- } \\
\text { wohnheiten agiere. }\end{array}$ & 3,9 & 4,07 & 0,17 \\
\hline
\end{tabular}

Wie in Tabelle $11 \mathrm{zu}$ sehen ist, liegen die Veränderungen im positiven Bereich, d.h. bei jedem Punkt hat sich der Stand der Kenntnisse verbessert. Zum Vergleich sind die Differenzen zwischen den Prä- und Posttests in den Kontrollgruppen nicht immer positiv. Der Zuwachs der Durchschnittswerte beträgt für die einzelnen Fragen 0,17 bis 1,23 Punkte. Um die Ergebnisse der EG zu veranschaulichen; wurde das folgende Diagramm erstellt. 
Abbildung 8: Veränderungen der Mittelwerte der einzelnen Aussagen zur Komponente „Kenntnisse“

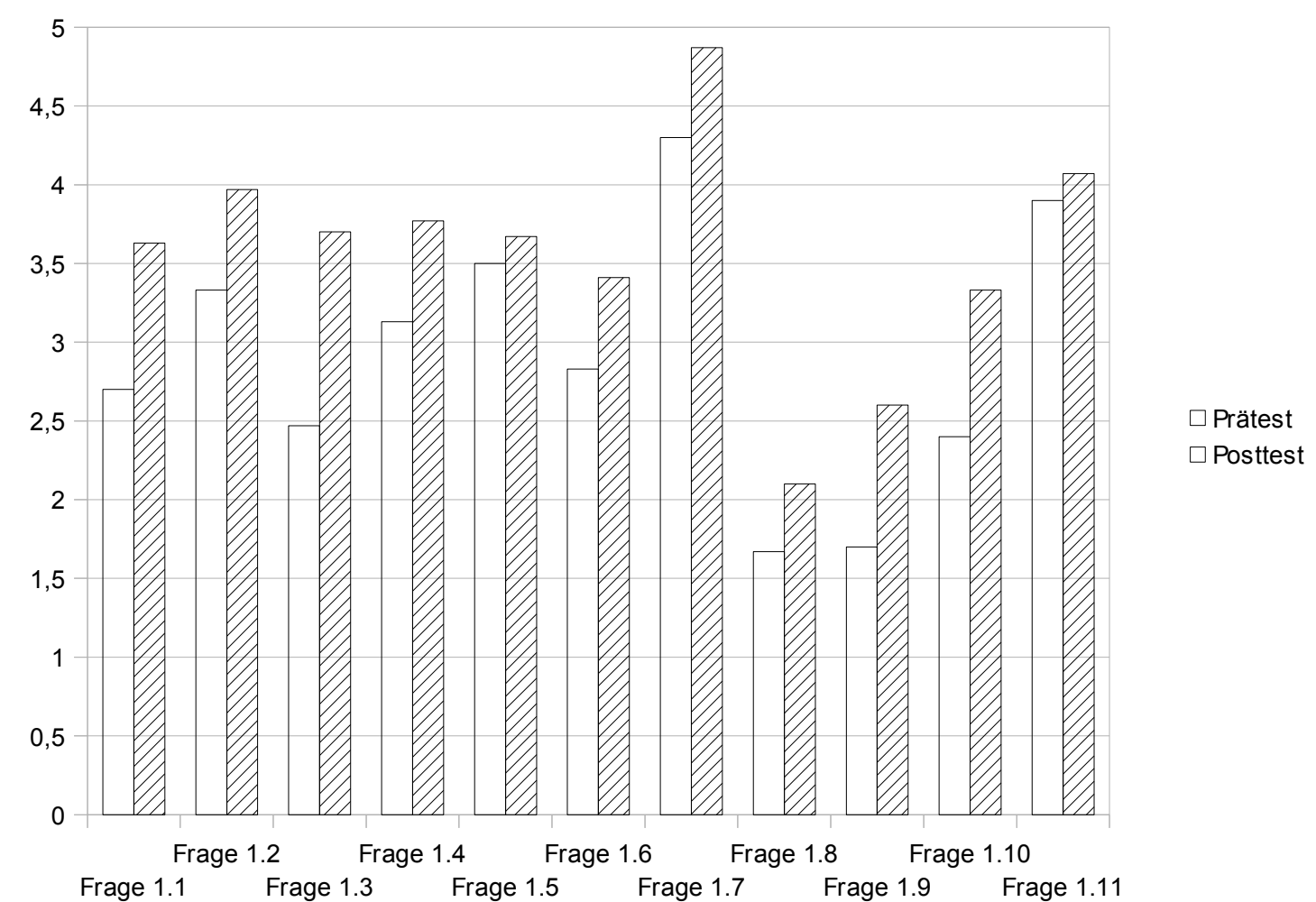

Die größten Fortschritte haben die Teilnehmer der EG in den Kenntnissen über die Kultur und Kulturtheorien gemacht, siehe Aussagen 1.1. und 1.3. In der Aussage 1.1. geht es um die Definition des Begriffs „Kultur“ und um die kulturelle Komponente, das Thema des ersten Blocks des PEIK, Hier steigen die Kenntnisse um 0,93 Punkte. In der Aussage 1.3 geht es um die Normen und Tabus der fremden Kultur, was sowohl innerhalb des PEIK als auch im Laufe des normalen Fremdsprachenunterrichts vermittelt wird. Hier steigt das Niveau der Kenntnisse am meisten um 1,23 Punkte.

Das Bewusstsein über wechselseitige Beziehungen des Fremden und des Eigenen und ihrer Auswirkung auf die interkulturelle Kommunikation zeigt ebenfalls positive Veränderungen. Die Aussage 1.9, die sich darauf bezieht, sowohl den speziellen kulturellen Hintergrund des fremdkulturellen Gesprächspartners als auch seine Auswirkung auf die Kommunikation zu erkennen, zeigt einen Zuwachs von 0,9 Punkten. Auch die Tatsache, dass jeder Teilnehmer von dem fremdkulturellen Partner anders wahrgenommen wird als ein Gesprächspartner gleicher Herkunft, wurde bewusster. Dieses Wissen über die veränderte Wahrnehmung seitens des Gesprächspartners wurde in der Frage 1.10 abgefragt, es erga- 
ben sich positive Veränderungen um 0,93 Punkte.

Dabei weist die kulturelle Selbstreflexion der Teilnehmer wenig positive Veränderungen auf. Obwohl in verschiedenen Übungen des PEIK versucht wurde, die Bewusstheit über die eigene kulturelle Prägung zu wecken und auf eigene kulturelle Einstellungen aufmerksam zu machen, zeigten die Mittelwerte zu den Aussagen 1.5 und 1.11 die geringsten Veränderungen, nur je 0.17 Punkte. Die Aussage 1.5, die sich auf die Hintergründe der eigenen kulturellen Einstellungen bezieht, wurde im Durchschnitt im Posttest nur um 0,17 Punkte besser beantwortet. Ein ähnliches Ergebnis ist bei der Aussage $1.11 \mathrm{zu}$ beobachten. Die Mittelwerte der Aussage zum Wissen um die eigene kulturelle Prägung steigen nur um 0,17 Punkte.

Aussage 1.8, die in beiden Tests die niedrigsten Bewertungen erhielt, die unmittelbar mit der Selbstreflexion der Teilnehmer verbunden ist und auf die Bewusstheit über ihre Emotionen und Gefühle während der interkulturellen Begegnung abzielt, zeigte ebenfalls positive, allerdings nur geringe Veränderungen. Die Bewusstheit der Teilnehmer steigt um 0,43 Punkte. Es fällt den Teilnehmern anscheinend schwer, über die affektive Seite des interkulturellen Kontakts nachzudenken. Mittelmäßig sind auch die Ergebnisse, nur ein Zuwachs von 0.59, zu der Aussage 1.6. Hier werden die Teilnehmer gefragt, ob sie die während einer interkulturellen Begegnung entstandenen Gefühle und Gedanken beschreiben können. Zusammenfassend lässt sich feststellen, dass es Veränderungen im kognitiven Bereich gab. Die Teilnehmer verbesserten sowohl ihre Kenntnisse über kulturelle Erscheinungen als auch ihre Bewusstheit über Besonderheiten der interkulturellen Kommunikation. Am besten haben sich die Kenntnisse über kulturelle Einstellungen und das Bewusstsein über das Phänomen der Andersartigkeit entwickelt. Dabei haben die Teilnehmer Probleme mit der Bewusstheit der eigenen kulturellen Prägung und mit den Kenntnissen über das eigene kulturelle „Ich“, d.h. den Teilnehmern fehlt immer noch die Fähigkeit zur Selbstreflexion über die eigene Kulturzugehörigkeit.

\subsubsection{Veränderungen in der Komponente ,interkulturelle Sensibilität““}

Die Entwicklung der interkulturellen Sensibilität ist ein lebenslanger Prozess, der mit der Entwicklung der Persönlichkeit eng verbunden ist. Bei der Entwicklung der interkulturellen Sensibilität sind zwar Veränderungen der Einstellungen zu erwarten, diese Veränderungen im affektiven Bereich bedürfen aber nicht nur theoretischer Arbeit, sondern vor allem 
praktischer Erfahrungen. Die während des Experiments mit Hilfe des PEIK durchgeführte Arbeit sollte durch regelmäßig stattfindende interkulturelle Begegnungen unterstützt werden. Diese Treffen zu organisieren und deren Einfluss auf die Einstellungen der Teilnehmer zu kontrollieren, hätte den Rahmen dieses Experiments gesprengt, deshalb wurde darauf verzichtet. Aus diesem Grund wurde in den Hypothesen zu dieser Arbeit vermutet, dass die Entwicklungen im Bereich ,interkulturelle Sensibilität“ nicht gravierend sein werden.

Abbildung 9: Prä-/Posttest-Vergleich zur Komponente „Sensibilität“: EG versus KG

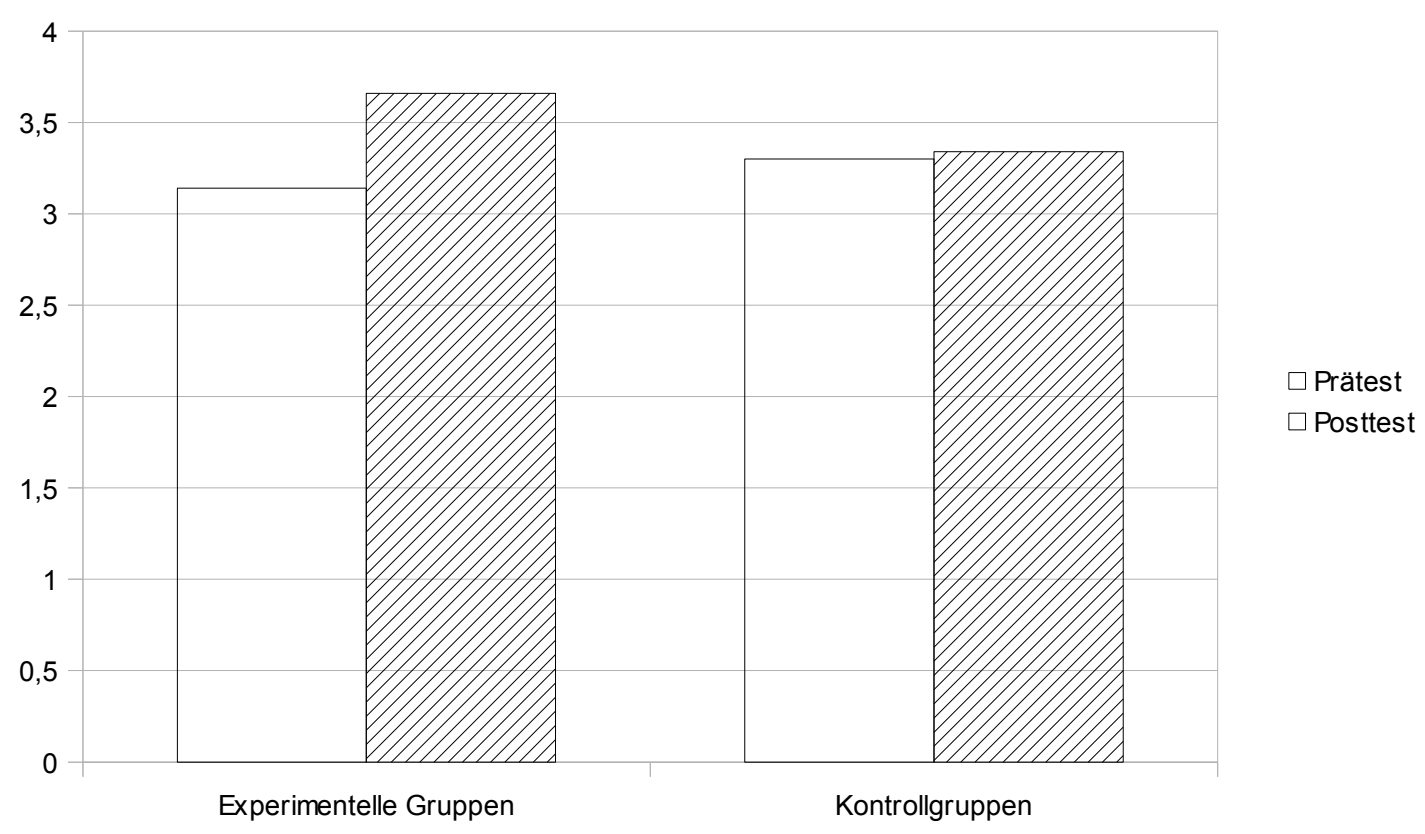

Abbildung 9 zeigt die Veränderungen im Bereich „Interkulturelle Sensibilität“‘ bei der Experimental- und Kontrollgruppe. In dem Diagramm ist der Unterschied zwischen den Ergebnissen der beiden Tests in den experimentellen Gruppen deutlich zu sehen (3,15 auf 3,66), in den Kontrollgruppen ist der Unterschied sehr gering (3,3 auf 3,34). Den Ergebnissen nach hat sich die interkulturelle Sensibilität der Teilnehmer der EG um 0,51 Punkte verbessert, dagegen schätzen die Teilnehmer der KG ihre Sensibilität in diesem Bereich unverändert ein.

Die Ergebnisse lassen feststellen, dass Veränderungen im affektiven Bereich innerhalb eines kurzen Zeitabschnitts möglich sind. Es widerlegt die oben angeführte Hypothese. Die Mittelwerte zur Komponente „Sensibilität“ der EG sind wesentlich höher im Posttest. Es stellt sich die Frage, welche Einstellungen veränderten sich zum Positiven. Diese Ergebnis- 
se werden in den folgenden Tabellen präsentiert.

Tabelle 12: Mittelwerte für die einzelnen Aussagen zur Komponente „Sensibilität“ in der Experimentalgruppe

\begin{tabular}{|c|c|c|c|}
\hline Aussagen & Prätest & Posttest & Differenz \\
\hline $\begin{array}{l}\text { 2.1. Ich bin zur Kommunikation mit Vertretern der anderen Kultur } \\
\text { bereit (ich vermeide sie nicht, ich ziehe den Kreis meiner } \\
\text { Landsleute nicht vor) }\end{array}$ & 3,73 & 4,17 & 0,43 \\
\hline 2.2. Ich interessiere mich für andere Sprachen und Kulturen. & 3,37 & 4,03 & 0,67 \\
\hline $\begin{array}{l}\text { 2.3. Ich bemühe mich, mich bei der Kommunikation mit dem } \\
\text { fremdkulturellen Partner entsprechend den Normen seiner } \\
\text { Kultur zu benehmen. }\end{array}$ & 3,30 & 4,00 & 0,70 \\
\hline $\begin{array}{l}\text { 2.4. Ich versuche die Gründe der negativen Emotionen, die im } \\
\text { Laufe der interkulturellen Kommunikation entstehen, zu ver- } \\
\text { stehen und zu lernen, mit innen umzugehen. }\end{array}$ & 2,83 & 2,93 & 0,10 \\
\hline $\begin{array}{l}\text { 2.5. Ich interessiere mich für solche Aspekte der anderen Kultur } \\
\text { wie Einstellungen, Verhaltensregeln, Geschichte und Tradi- } \\
\text { tionen. }\end{array}$ & 3,33 & 3,67 & 0,33 \\
\hline $\begin{array}{l}\text { 2.6. Ich bin bereit, mein eigenes Verhalten entsprechend den } \\
\text { Normen der fremden Kultur zu ändern (Sprache, Körper- } \\
\text { sprache, Verhaltensregeln in verschiedenen Situationen). }\end{array}$ & 2,43 & 3,13 & 0,70 \\
\hline $\begin{array}{l}\text { 2.7. Ich bin bereit, über die Auswirkungen meines Verhaltens } \\
\text { und meiner Worte auf meinen fremdkulturellen Partner } \\
\text { nachzudenken. }\end{array}$ & 3,00 & 3,67 & 0,67 \\
\hline
\end{tabular}

Wie aus der Tabelle zu ersehen ist, sind die Differenzen zwischen den Mittelwerten des Prä- und Posttests ausschließlich positiv, sie liegen zwischen 0,4 und 0,7 Punkten, niedriger lediglich die zu Frage 4. Die Ergebnisse veranschaulicht das folgende Diagramm. 
Abbildung 10: Veränderungen der Mittelwerte zu den einzelnen Aussagen zur Komponente „interkulturelle Sensibilität“

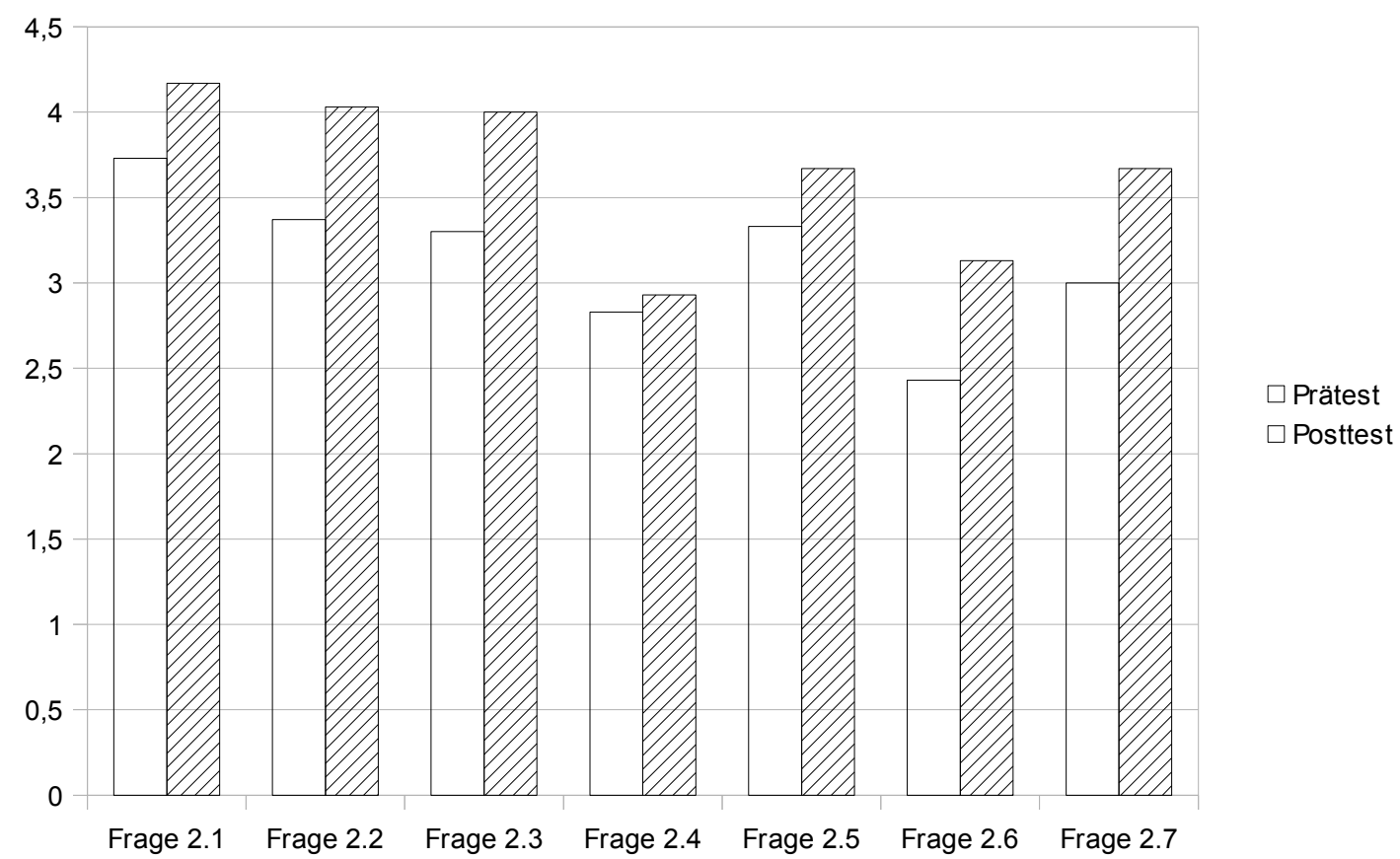

Die Teilnehmer der EG bekunden ein gestiegenes Interesse an fremden Sprachen und Kulturen. Die Mittelwerte zur Aussage 2.2 sind um 0,7 Punkte gestiegen, was auch vermuten lässt, dass die Motivation der Teilnehmer im Fremdsprachenunterricht durch zusätzliches interkulturelles Material verbessert werden kann. Dass ihnen aber die interkulturellen Inhalte noch relativ „fremd“ sind, zeigt ihre Selbsteinschätzung zur Frage 2.5. Ihr Interesse für spezifische interkulturelle Begriffe ist zwar gestiegen, aber wesentlich weniger als ihr allgemeines Interesse. Die Differenz zwischen den Mittelwerten zur Aussage über das Interesse an fremdkulturellen Einstellungen, Verhaltensregeln, Traditionen und Geschichte beträgt 0,33 Punkte.

Was die Inhalte des Programms bewirken konnten, sind solche Einstellungen wie Empathie und eine eng damit verbundene Ambiguitätstoleranz. Die Teilnehmer erklären sich bereit, sich in die Welt des fremdkulturellen Gesprächspartners einzufühlen und entsprechend seinen Normen zu handeln. Das bestätigt Aussage 2.3: das Empathievermögen ist um 0,7 Punkte gestiegen. Genauso sehen die Teilnehmer der EG die Notwendigkeit, das eigene Verhalten entsprechend der fremden Normen zu steuern, d.h. sie sind bereit, die eigenen kulturellen Normen zu relativieren. Das bestätigen die Ergebnisse zur Aussage 2.6, die eine 
positive Differenz von 0,7 Punkten zwischen dem Prä- und dem Posttests zeigen.

Leider funktioniert die Selbstreflexion der Teilnehmer der EG nicht in allen Richtungen so gleichmäßig. Wenn es darum geht, die eigenen Gefühle, besonders die negativen, zu reflektieren und zu akzeptieren, scheitern die Teilnehmer. Wie schon im Prätest erkennen die Teilnehmer auch im Posttest nicht an, dass negative Gefühle, ebenso wie Spannungen und Missverständnisse, in einer interkulturellen Situation unausweichlich sind. Mittelwerte zur Aussage 2.4 zeigten kaum Differenzen zwischen Prä- und Posttest.

\subsubsection{Veränderungen in der Komponente ,interkulturelle Fähigkeiten“}

Die verhaltensbezogene Komponente der interkulturellen Kompetenz schließt die Fähigkeit ein, mit Hilfe von Kenntnissen und interkultureller Sensibilität adäquat und erfolgreich in Situationen interkultureller Kontakte zu kommunizieren. Auf der Handlungsebene kommen zweierlei Teilfähigkeiten zum Tragen: sowohl auf die handelnde Person bezogen als auch auf den fremdkulturellen Partner gerichtet. Erfolgreich zu agieren bedeutet in diesem Zusammenhang nicht nur Erreichen der eigenen Ziele, sondern auch Achtung des Kommunikationspartners.

Die interkulturellen Fähigkeiten brauchen für ihre Entwicklung die praktischen Erfahrungen. Kenntnisse können theoretisch erlernt oder durch Nachdenken angeeignet werden, durch Auseinandersetzung mit interkulturellen Inhalten kann die Sensibilität entwickelt werden, die Entwicklung der Fähigkeiten bedarf der Praxis. Aus diesem Grund wurde in den Thesen zu dieser Arbeit davon ausgegangen, dass sich nach dem Einsatz des PEIK die interkulturellen Fähigkeiten der Teilnehmer nur wenig verändern, da im Rahmen des Fremdsprachenunterrichts keine authentischen Kontakte mit Vertretern der fremdsprachlichen Kultur organisiert werden konnten.

In Diagramm 5 sind die Veränderungen im Bereich ,,interkulturelle Fähigkeiten“ für EG und $\mathrm{KG}$ veranschaulicht. 
Abbildung 11: Prä-/Posttest-Vergleich zur Komponente „Fähigkeiten“: EG versus KG

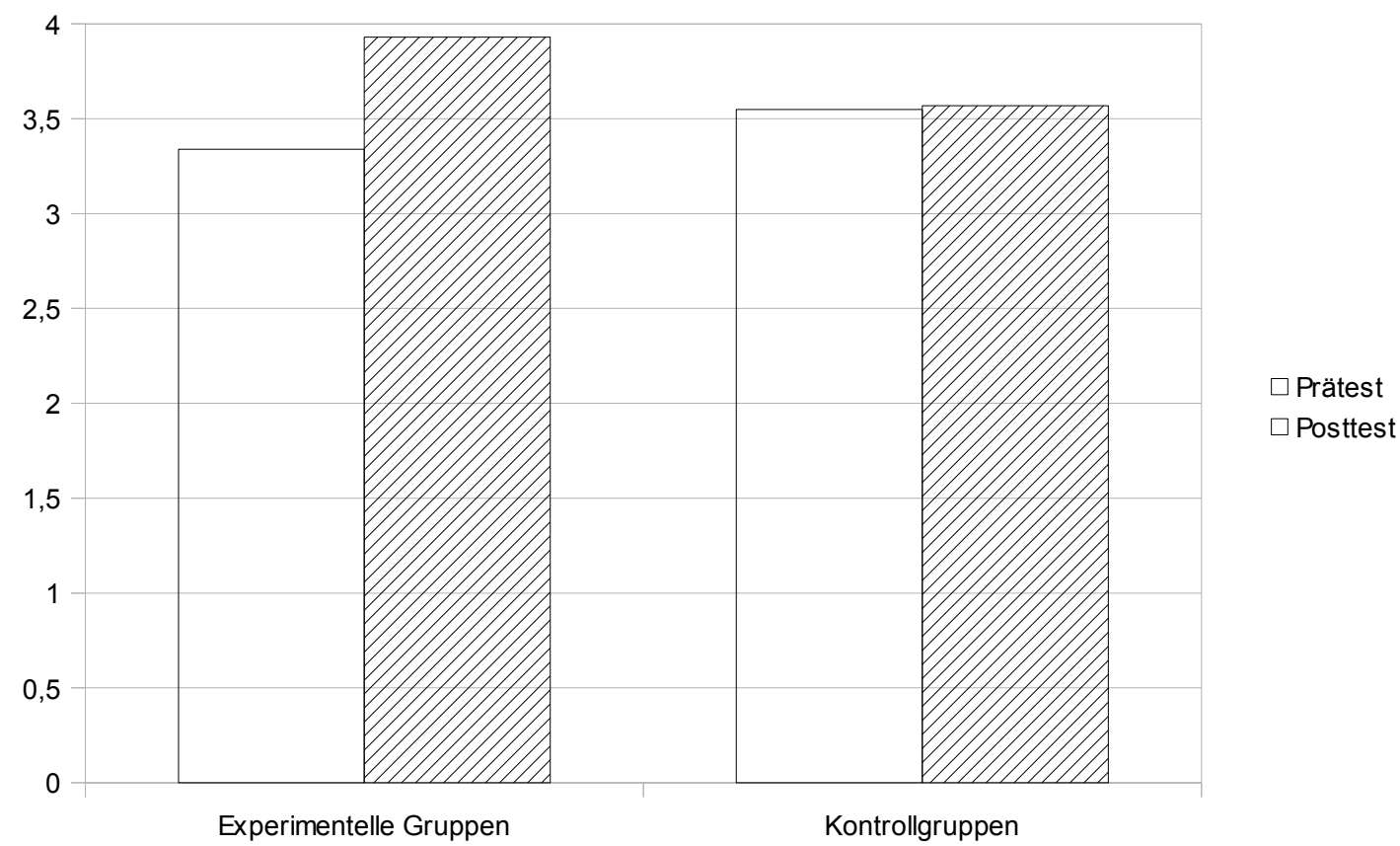

Trotz der oben aufgestellten These sind die Veränderungen in den experimentellen Gruppen ganz deutlich zu sehen (3,34 auf 3,93). Dabei sind die Ergebnisse der Kontrollgruppen wie erwartet auf dem gleichen Niveau geblieben $(3,55$ auf 3,57). Die Differenz in den experimentellen Gruppen erreicht 0,6 Punkte. Die Differenz von 0,2 Punkten in den Kontrollgruppen kann vernachlässigt werden.

In der folgenden Tabelle sind die Veränderungen innerhalb der einzelnen Fragen zusammengefasst.

Tabelle 13: Mittelwerte für die einzelnen Aussagen zur Komponente „Fähigkeiten“ in der Experimentalgruppe

\begin{tabular}{|l|r|r|r|}
\hline Aussagen & \multicolumn{1}{|c|}{ Prätest } & \multicolumn{1}{|c|}{ Posttest } & Differenz \\
\hline $\begin{array}{l}\text { 3.1. Ich bin flexibel bei der Kommunikation mit dem fremdkultu- } \\
\text { rellen Partner. }\end{array}$ & 2,87 & 3,67 & 0,80 \\
\hline $\begin{array}{l}\text { 3.2. Ich kommuniziere entsprechend den Regeln der fremden } \\
\text { Kultur, damit ich meinen fremdkulturellen Partner durch mein } \\
\text { Verhalten, meine Kleidung etc. nicht beleidige. }\end{array}$ & 3,13 & 3,90 & 0,77 \\
\hline $\begin{array}{l}\text { 3.3. Ich kann meine eigene und die fremde Kultur miteinander } \\
\text { vergleichen. }\end{array}$ & 4,30 & 4,50 & 0,20 \\
\hline
\end{tabular}




\begin{tabular}{|l|r|r|r|}
\hline $\begin{array}{l}\text { 3.4. Bei der Kommunikation mit den Vertretern einer fremden } \\
\text { Kultur benutze ich Strategien zur Verringerung von Kultur- } \\
\text { schock7k. }\end{array}$ & 2,50 & 2,83 & 0,33 \\
\hline $\begin{array}{l}\text { 3.5. Ich kann meine Fremdsprachen- und Fremdkulturkenntnisse } \\
\text { verbessern. }\end{array}$ & 3,80 & 4,23 & 0,43 \\
\hline $\begin{array}{l}\text { 3.6. Ich benutze eine Vielfalt von Verhaltensstrategien, wenn ich } \\
\text { mit Leuten mit einem anderen kulturellen Hintergrund kom- } \\
\text { muniziere. }\end{array}$ & 2,43 & 3,63 & 1,20 \\
\hline $\begin{array}{l}\text { 3.7. Ich bin fähig, passende Verhaltensstrategien zu finden, } \\
\text { wenn bei der interkulturellen Kommunikation etwas falsch } \\
\text { gelaufen ist. }\end{array}$ & 3,97 & 0,63 \\
\hline $\begin{array}{l}\text { 3.8. Ich kann aufgrund der sozialpolitischen Faktoren erklären, } \\
\text { was meine Kultur von der fremden unterscheidet. }\end{array}$ & 3,70 & 4,10 & 0,40 \\
\hline
\end{tabular}

Wie zu sehen ist, sind alle Veränderungen in den EG positiv, d.h. die Inhalte des PEIK haben zur Verbesserung der interkulturellen Fähigkeiten nach der Selbsteinschätzung der Teilnehmer geführt. Das Niveau der Veränderungen variiert abhängig von der Frage und liegt im Bereich von 0,2 bis 1,2 (siehe Diagramm 6).

Abbildung 12: Veränderungen der Mittelwerte der einzelnen Fragen zur Komponente „Fähigkeiten"

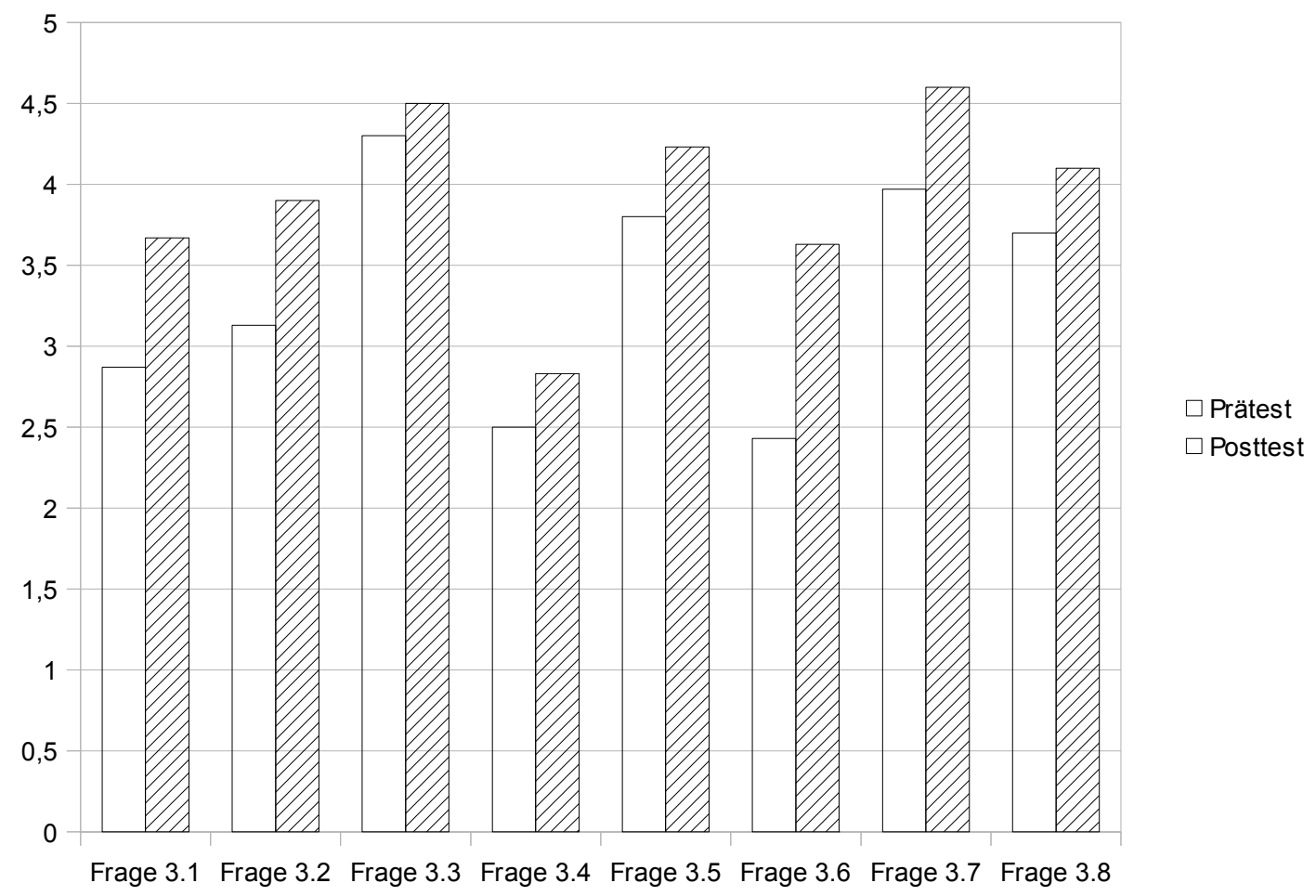


Den höchsten Grad an Veränderungen innerhalb dieses Experiments (1,2 Punkte) hat die Selbsteinschätzung der Teilnehmer zur Aussage 3.6 über die benutzten Verhaltensstrategien innerhalb der interkulturellen Kommunikation. Bei dieser Aussage schätzen sie ihre Fähigkeit zur Vermeidung von Missverständnissen ein. Es ist bemerkenswert, dass die Ergebnisse zu dieser Aussage im Prätest eine der niedrigsten war; im Posttest zeigen die Teilnehmer ein mittleres Niveau im Vergleich zu den anderen Fähigkeiten. Dabei war die Aussage 3.7 über die Fähigkeit zur Vermeidung von Missverständnissen schon im Prätest relativ hoch $(3,97)$ bewertet, diese Fähigkeit hat sich aber trotzdem nach dem Experiment um 0,6 Punkte verbessert. Diese zwei Teilfähigkeiten, die im Puzzle-Modell zur Fähigkeit zur Vermeidung und Klärung von Missverständnissen zusammengefasst sind, haben sich gut entwickelt. Dabei haben die Teilnehmer Folgendes gelernt: Nicht nur bei misslungener oder fehlgelaufener Kommunikation ist ihr Einsatz wichtig. Vielmehr werden Selbstreflexion, Flexibilität und Empathie benötigt, um Fehler bei der interkulturellen Kommunikation zu vermeiden und sie befriedigend für beide Seiten in Gang zu halten.

Positive Veränderungen zeigt auch die Kommunikationsfähigkeit der Teilnehmer. Da die Sprachkenntnisse in diesem Test nicht gemessen werden, wird hier über außersprachliche Erscheinungen wie nonverbale Elemente, Flexibilität, Dressingcode, Regeln für soziale Beziehungen gesprochen. So geht es bei Aussage 3.1 um Flexibilität bei der interkulturellen Kommunikation, die Prä-Posttestdifferenz beträgt 0,8 Punkte. Bei der Aussage 3.2, Kommunikationsregeln mit dem fremdkulturellen Partner, beträgt sie 0,77. Dies zeigt, dass die Teilnehmer gelernt haben, nicht nur die sprachliche Seite der Kommunikation, sondern auch die oft verdeckte Seite, die durch die Kultur der Teilnehmer bedingt ist, zu sehen und sie bei der Kommunikation zu beachten.

Leider bleibt die Entwicklung der Fähigkeit zur konstruktiven Auseinandersetzung mit Fremdem und Eigenem auf einem mittelmäßigen Niveau. Diese Fähigkeit, die untrennbar mit der Fähigkeit zur Selbstreflexion verbunden ist, zeigt die wenigsten Veränderungen. So schätzen die Teilnehmer in Aussage 3.3 ihre Fähigkeit, die eigene mit einer fremden Kultur zu vergleichen, im Posttest nur 0,2 Punkte besser als im Prätest. Die Aussage 3.8 über die Fähigkeit, aufgrund der sozialen oder politischen Erscheinung die Unterschiede zu erklären, wurde im Posttest um 0,4 Punkte besser bewertet. Dies liegt vielleicht daran, dass das Entwicklungsniveau dieser Fähigkeit schon vor dem Anfang des Experiments relativ hoch war, so dass es wenig Spielraum für den weiteren Fortschritt geblieben ist.

Auf mittelmäßigem bis niedrigem Niveau liegen die Veränderungen in der Lernfähigkeit und in der Fähigkeit zur Verringerung von Kulturstress. Das ist bei den Aussagen 3.4 und 
3.5 zu sehen. Die Differenz zwischen den Werten des Prä- und Posttests zur Aussage 3.4 über die Strategien zur Verringerung des Kulturstresses beträgt 0,3, zur Aussage 3.5 über die Fähigkeit zur Verbesserung der Fremdsprachen- und Kulturkenntnisse 0,4. Das dürfte daran liegen, dass diese beiden Fähigkeiten nicht im Inhalt des Programms eingeschlossen waren, d.h. es wurde im PEIK nicht vermittelt, dass die Teilnehmer selbstständig ihre kulturellen Kenntnisse erweitern können. Auf das Thema „Kulturstress“ wurde auch nicht eingegangen.

\subsection{Prä- und Posttest-Vergleich - Gesamtergebnis}

Im folgenden Kapitel werden die Gesamtergebnisse präsentiert und auf die Signifikanz mithilfe des T-Tests für gepaarte Variablen überprüft. Im Weiteren werden die Ergebnisse bezüglich einiger unabhängiger Daten über die Teilnehmer des Experiments bewertet. Hier soll geprüft werden, wie Art und Dauer des Fremdkulturkontakts, Sprachleistung, Geschlecht und Kurszugehörigkeit die interkulturelle Kompetenz und den Lerngewinn beeinflussen. Anzunehmen ist, dass größere Vorerfahrung (1.) sich bereits durch höhere Werte im Vortest für alle Komponenten abbildet; (2.) Lerngewinne in allen drei Kompetenzbereichen durch die Kursteilnahme zeitigt und im Prä-Posttest-Vergleich abbildet; (3.) dass größere Vorerfahrung sich vor allem im Handlungsbereich abbildet - sowohl vor als auch nach dem Kurs. Ob das Geschlecht der Teilnehmer einen Einfluss auf die Entwicklung der interkulturellen Kompetenz hat, soll als offene Frage diskutiert werden, ebenso mögliche Gründe für Unterschiede zwischen den vier untersuchten Kursgruppen.

\subsubsection{Gesamtergebnis: interkulturelle Kompetenz - Zusammenfassung}

In der Abbildung 13 sind die Gesamtergebnisse des Experiments angezeigt. Dort wird sichtbar, dass sich das Niveau der interkulturellen Kompetenz der Experimentteilnehmer positiv verändert hat, im Durchschnitt von 3,13 im Prätest auf 3,72 im Posttest, also um 0,59 Punkte. Die Ergebnisse der Kontrollgruppe zeigen dagegen 3,31 in Prä- und 3,28 im Posttest. 
Abbildung 13: Prä-/Posttest-Vergleich zum Gesamtergebnis ,interkulturelle Kompetenz“: EG versus $\mathrm{KG}$

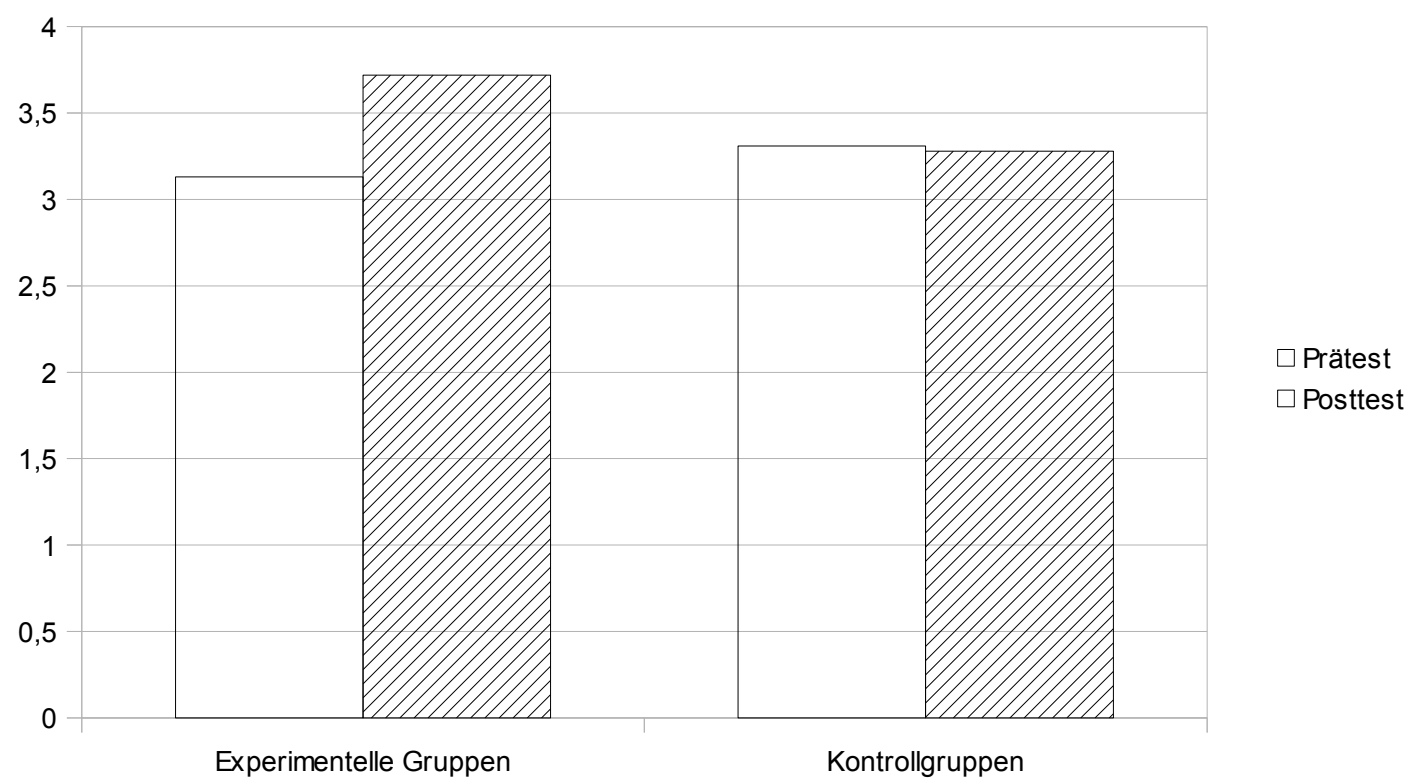

Im Kapitel 7.3. wurden die Mittelwerte für die experimentellen Gruppen bezüglich der Bestandteile der interkulturellen Kompetenz im Einzelnen analysiert. In den folgenden Tabellen werden diese Werte zusammengefasst.

Tabelle 14: Mittelwerte und Mittelwertdifferenzen für die drei Komponenten der interkulturellen Kompetenz in der Experimentalgruppe $(n=30)$

\begin{tabular}{|l|r|r|r|}
\hline Komponente der IK & Prätest & Posttest & \multicolumn{2}{l|}{$\begin{array}{l}\text { Mittelwertdiffe- } \\
\text { renz }\end{array}$} \\
\hline Kenntnisse & 2,90 & 3,56 & 0,65 \\
\hline Interkulturelle Sensibilität & 3,14 & 3,66 & 0,51 \\
\hline Interkulturelle Fähigkeiten & 3,34 & 3,93 & 0,60 \\
\hline
\end{tabular}

Wie in Tabelle $14 \mathrm{zu}$ sehen ist, sind die Verbesserungen im Bereich „Kenntnisse“ und „Fähigkeiten" etwas deutlicher als im affektiven Bereich. Dabei war Ausgangsniveau der Kenntnisse niedriger als dieses von der Sensibilität oder Fähigkeiten. Das ist auch der Grund, warum die interkulturellen Fähigkeiten bei den Endergebnissen besser entwickelt sind als Kenntnisse und die interkulturelle Sensibilität. Zur Veranschaulichung wird hier 
die folgende Grafik präsentiert.

Abbildung 14: Prä-/Posttest-Vergleich: Veränderungen zu den einzelnen Komponenten der interkulturellen Kompetenz in der EG

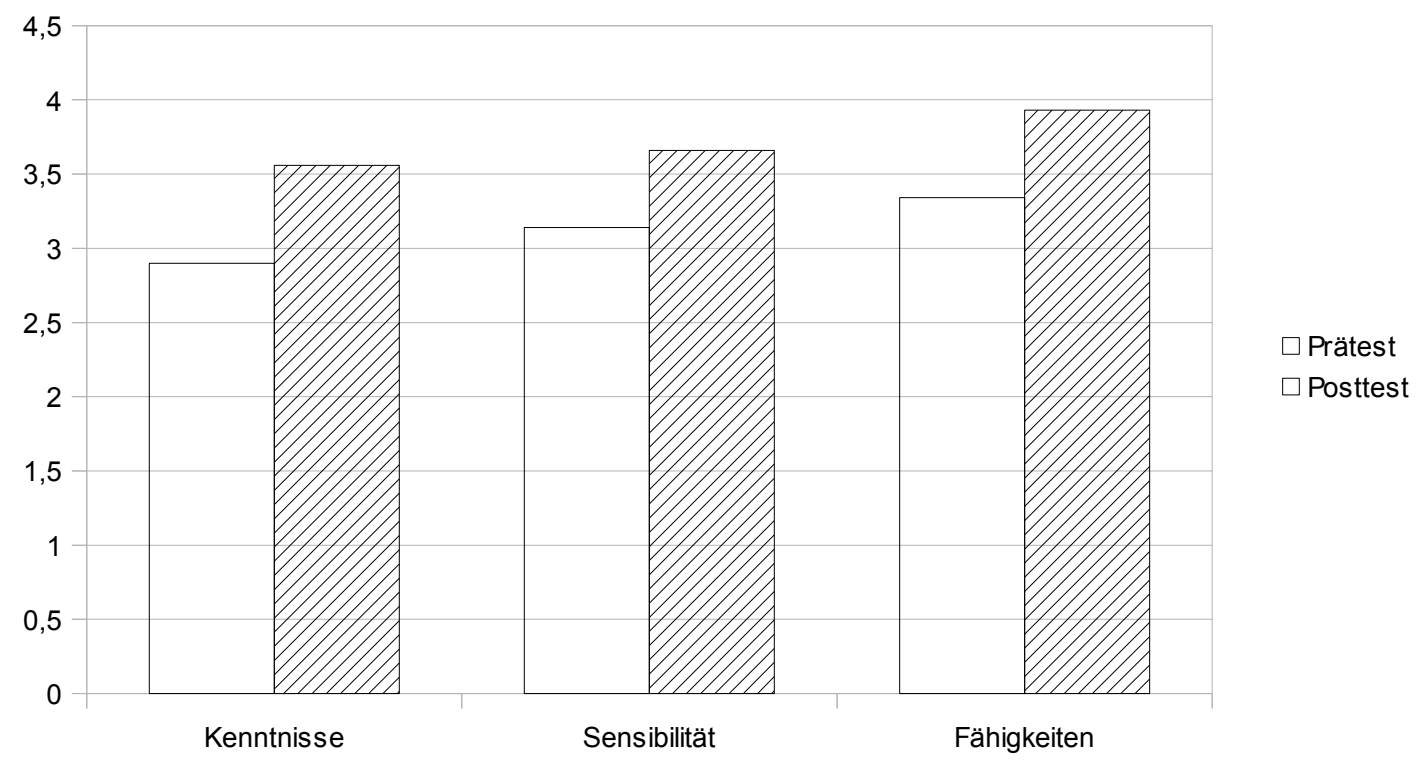

\subsubsection{Gesamtergebnis: Signifikanzüberprüfung}

Die Ergebnisse wurden mithilfe des T-Tests für gepaarte Stichproben auf Signifikanz überprüft.

Tabelle 15: T-Test und Signifikanzprüfung der Ergebnisse des Prä- und Posttestvergleichs in den EG

\begin{tabular}{|l|l|l|l|l|l|l|}
\hline & \multicolumn{3}{|c|}{ Gepaarte Differenzen } & T & $\begin{array}{c}\text { Sig. (2- } \\
\text { seitig) }\end{array}$ \\
\hline & Mittelwert & $\begin{array}{l}\text { Standardab } \\
\text {-weichung }\end{array}$ & $\begin{array}{l}\text { 95\% Konfidenzintervall } \\
\text { der Differenz }\end{array}$ & & \\
\hline & & & Untere & Obere & & \\
\hline $\begin{array}{l}\text { Kenntnisse } \\
\text { Sensibilität }\end{array}$ & 0,65 & 0,46 & 0,46 & 0,82 & 7,82 & 0,000 \\
Fähigkeiten & 0,6 & 0,61 & 0,29 & 0,74 & 4,64 & 0,000 \\
& 0,57 & 0,38 & 0,81 & 5,7 & 0,000 \\
\hline
\end{tabular}

Bei allen drei Komponenten beträgt das Signifikanzniveau 0,000, das bedeutet, dass das Ergebnis hochsignifikant ist. Für die vorliegende Untersuchung kann dieses Ergebnis fol- 
gendermaßen interpretiert werden: Die Wahrscheinlichkeit, dass die Inhalte von PEIK positiv die Entwicklung aller drei Komponenten der interkulturellen Kompetenz beeinflusst haben, ist sehr hoch. Damit wird die in Kapitel 6.1. aufgestellte Hypothese bestätigt. Zum Vergleich wird das Signifikanzniveau der Ergebnisse in den Kontrollgruppen gerechnet.

Tabelle 16: T-Test und Signifikanzüberprüfung der Ergebnisse des Prä- und Posttestvergeleich in den $\mathrm{KG}$

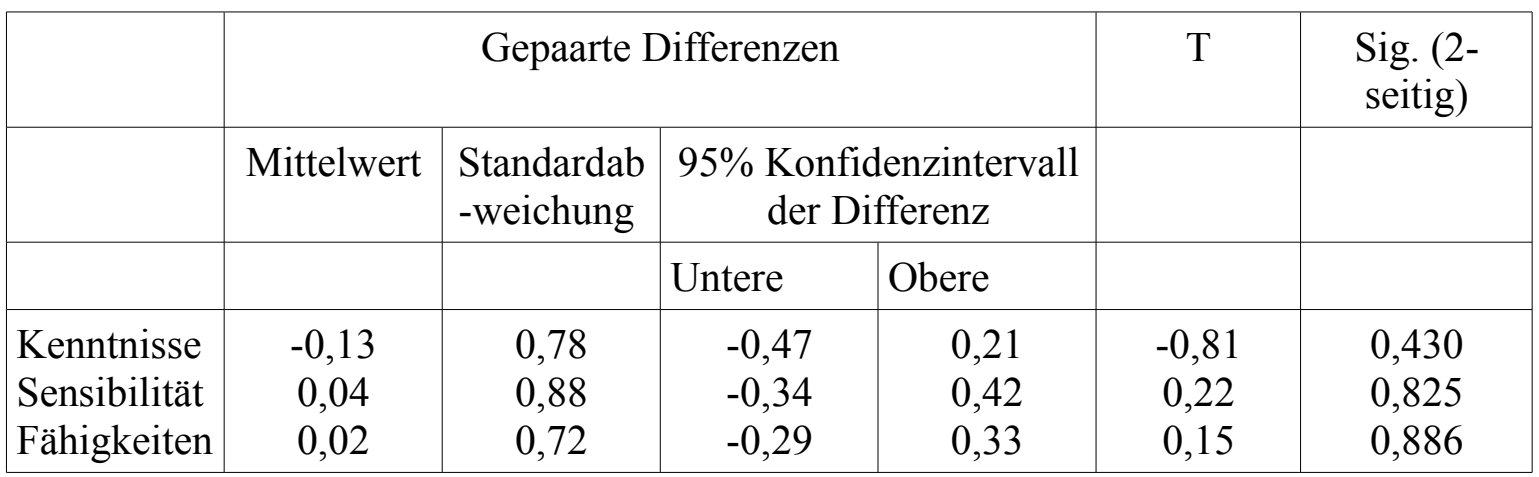

Wie aus der Tabelle ersichtlich ist, zeigen sich Signifikanzniveaus aller Komponenten $>0,05$. Das bedeutet, dass die Mittelwertdifferenzen zwischen den Ergebnissen des Präund Posttests in den Kontrollgruppen keinen wesentlichen Unterschied aufweisen und deshalb nicht signifikant sind.

\subsubsection{Vergleich der Veränderungen in den Gruppen mit unterschiedlichen Ausgangsbedingungen}

Im Weiteren werden die Gesamtergebnisse des Experiments bezüglich einiger Daten über die Teilnehmer präsentiert und analysiert.

\section{Interkulturelle Kompetenz und Kurszugehörigkeit}

An dem Experiment haben die zwei im Kapitel 6.3.2. beschriebenen Gruppen teilgenommen. Die Veränderungen der interkulturellen Kompetenz sind in beiden Gruppen gleich und betragen 0,58 Punkte. Dabei war das Ausgangsniveau der EG1 höher, die Endergebnisse dieser Gruppe liegen im Bereich 3,92, die der EG2 sind 3,53 Punkte (siehe folgende Abbildung). 
Abbildung 15: Prä-/Posttest-Vergleich: Veränderungen der interkulturellen Kompetenz in den EG: EG1 vs. EG2

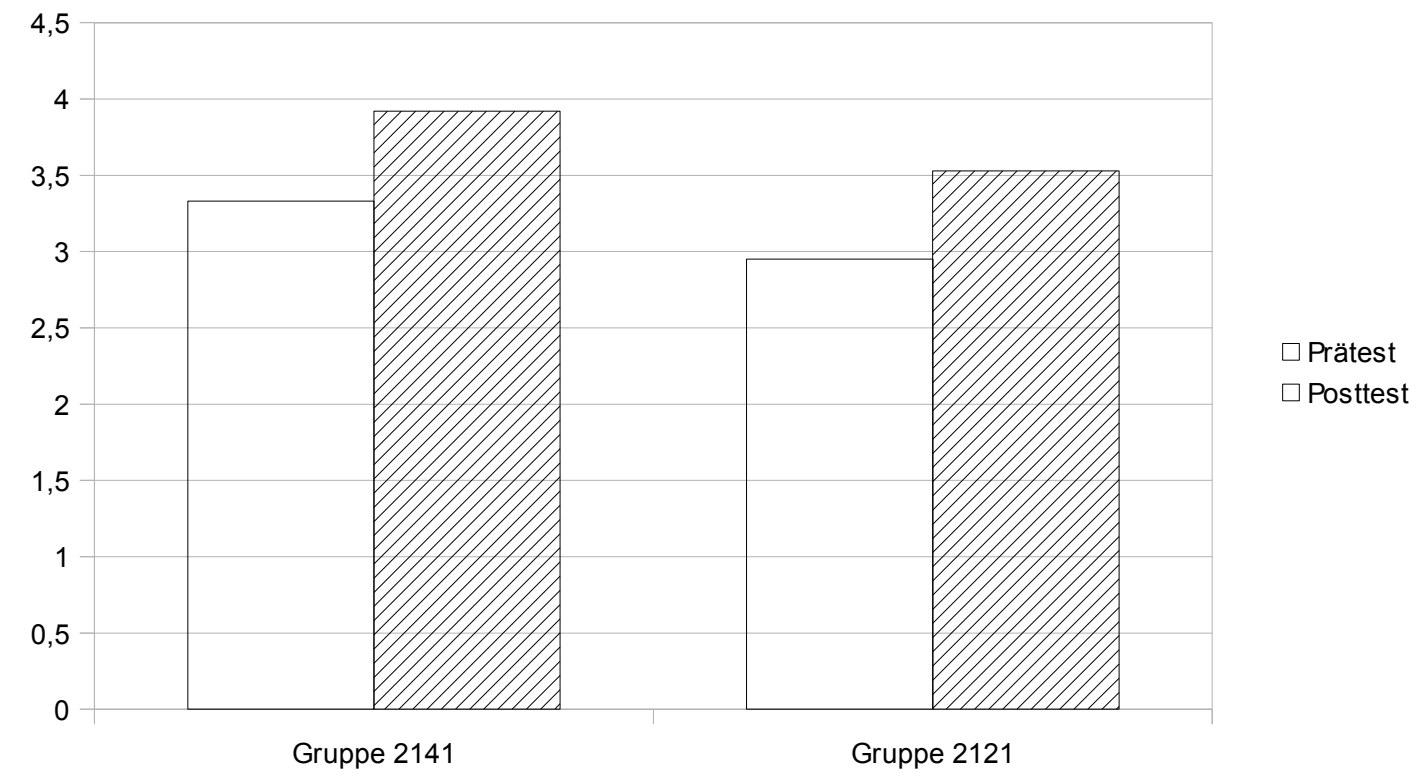

Wie aus Kapitel 6.3.2. folgt, waren die Durchschnittsleistungen in der Fremdsprache der EG1 besser als die der EG2. Es ergibt sich die Schlussfolgerung, dass der Lernerfolg im Fremdsprachenunterricht auch die Erfolge in der Entwicklung der interkulturellen Kompetenz beeinflusst. Dabei ist aber zu bemerken, dass die EG2 eine gemischte nationale Zusammensetzung hatte, d.h. von 16 Teilnehmern waren fünf Teilnehmer burjatischer und 11 russischer Herkunft. Es wäre zu erwarten gewesen, dass die interkulturelle Kompetenz dieser Gruppe im Durchschnitt höher sein sollte, da die Burjaten ihr Leben bikulturell und manchmal bilingual gestalten. Diese Annahme wurde aber nicht bestätigt.

\section{Interkulturelle Kompetenz und Geschlecht}

Von den 30 Teilnehmern am Experiment waren 27 Männer. Wegen dieser Ungleichverteilung der Geschlechter sind statistische Vergleiche nicht sinnvoll, dennoch einige Anmerkungen zur Variable Geschlecht im Experiment. Bei gleicher Ausgangslage vor Kursbeginn, hatten die drei Frauen besonders große Fortschritte bei der Entwicklung der interkulturellen Kompetenz gemacht. Die Durchschnittsnote im Posttest hat sich bei den Männern um 0,56, bei den Frauen um 0,81 verbessert. Größere Lernerfolge von Frauen beim Sprachenlernen sind in der Literatur häufig belegt. Ob die Frauen erfolgreicher in der Entwick- 
lung der interkulturellen Kompetenz sind, bleibt hier offen. Die Verfasserin hat folgende Erfahrungen gemacht: An der Ost-Sibirischen Hochschule des MdI studieren sehr zielstrebige und lernfähige Frauen. Sie erlernen einen „männlichen“ Beruf und sind meist dazu gezwungen, sich in einem männlichen Studienkollektiv durchzusetzen. Dazu nutzen sie Lernerfolge im Studium. Dies dürfte auch für die Entwicklung der interkulturellen Kompetenz relevant sein.

Abbildung 16: Prä-/Posttest-Vergleich: Veränderungen der interkulturellen Kompetenz in den EG nach Geschlecht

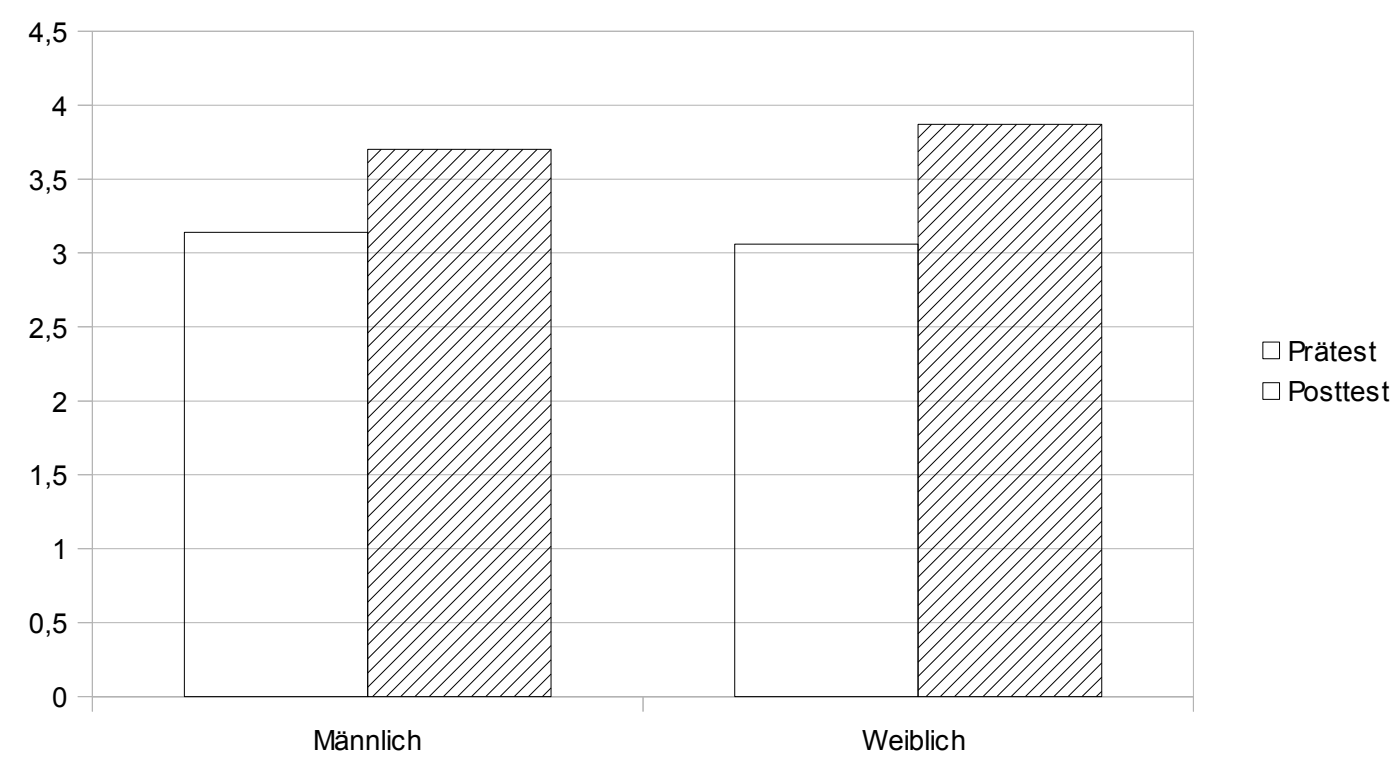

\section{Interkulturelle Kompetenz und Auslandserfahrungen}

Im Einführungsteil des Fragebogens wird die Frage: „Waren Sie schon im Ausland?“. mit drei Antwortalternativen gestellt: ,ja, weniger als 3 Monate“, ,ja, mehr als 3 Monate“, „nein“. Hier wurde bewusst nicht nach interkulturellen Erfahrungen, die ja innerhalb des eigenen Landes durchaus möglich sind, gefragt, sondern nach Auslandserfahrungen, nämlich der tatsächlichen Konfrontation mit einer anderen Kultur. Von 30 Experimentteilnehmern gaben nur vier an, in einem anderen Land gewesen zu sein, und zwar alle weniger als drei Monate. Es handelt sich dabei also um kurzfristige Aufenthalte, etwa eine Urlaubsreise, einen kurzen Gastbesuch oder eine Studienreise. Zwei Fragen sind zu prüfen: 1. Erzielen Studierende der KG mit Auslandserfahrung, selbst wenn sie nur kurzfristig ist, höhere IK-Werte, und 2. Haben die Erfahrenen durch das Experiment einen größeren Gewinn als 
andere Kursanten?

Abbildung 17: Prä-/Posttest-Vergleich: Veränderungen der interkulturellen Kompetenz in den EG nach Auslandserfahrungen

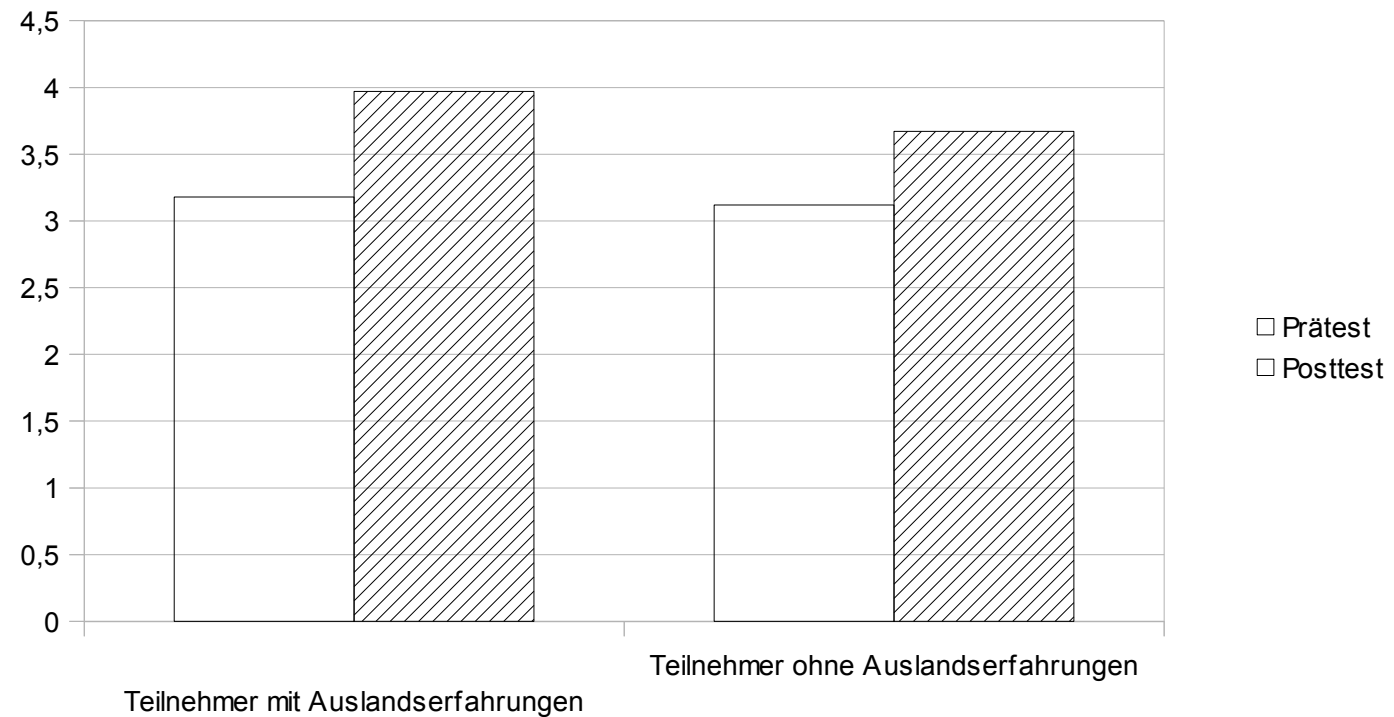

Wegen der geringen Anzahl ist eine statistische Überprüfung nicht angebracht, dennoch zeigt das Diagramm, dass bei fast gleichen Ausgangsbewertungen (3,18 für die Teilnehmer mit Auslandserfahrungen und 3,12 für die Teilnehmer ohne solche Erfahrungen) die interkulturelle Kompetenz der Teilnehmer mit Auslandserfahrungen nach dem Experiment ein höheres Niveau $(3,97)$ erreicht als die der Teilnehmer ohne Auslandserfahrungen $(3,68)$.

Tabelle 17: Mittelwerte und Mittelwertdifferenzen für die drei Komponenten der interkulturellen Kompetenz in der EG $(\mathrm{n}=30)$ : Teilnehmer mit geringeren Auslandserfahrungen vs. Teilnehmer ohne Auslandserfahrungen

\begin{tabular}{|l|c|c|c|c|c|c|}
\hline & \multicolumn{3}{|c|}{ Ja, weniger als 3 Monate } & \multicolumn{3}{c|}{ Nein } \\
\hline & Prätest & Posttest & Differenz & Prätest & Posttest & Differenz \\
\hline Kenntnisse & 2,93 & 3,89 & 0,95 & 2,90 & 3,51 & 0,61 \\
\hline $\begin{array}{l}\text { Interkulturelle } \\
\text { Sensibilität }\end{array}$ & 3,04 & 3,96 & 0,93 & 3,16 & 3,61 & 0,45 \\
\hline $\begin{array}{l}\text { Interkulturelle Fä- } \\
\text { higkeiten }\end{array}$ & 3,56 & 4,06 & 0,50 & 3,30 & 3,91 & 0,61 \\
\hline
\end{tabular}


Unabhängig von den Auslandserfahrungen zeigten beide Gruppen der Teilnehmer ähnliche Ergebnisse im Prätest. Anders sieht es im Posttest aus. Die erfahreneren Teilnehmer haben ihre Qualitäten in den Bereichen „Kenntnisse“ und „Sensibilität“ eindeutig stärker verbessert als unerfahrene Teilnehmer. Besonders gravierend ist dieser Unterschied bei der interkulturellen Sensibilität. Zusammenfassend kann gesagt werden, dass Auslandserfahrungen keine Garantie für eine besser entwickelte interkulturelle Kompetenz sind. Allerdings verbessern sich die interkulturellen Kompetenzen bei der gezielten Arbeit an diesem Konstrukt, wie es im PEIK erfolgt.

\section{Interkulturelle Kompetenz und interkulturelle Kontakte}

Eine andere Möglichkeit, über interkulturelle Erfahrungen der Teilnehmer etwas zu erfahren, sind die Angaben zur Frage „Haben Sie Freunde aus anderen kulturellen Kreisen?“ Bei dieser Frage geht es um interkulturelle Kontakte innerhalb der eigenen Kultur. Dabei sind nicht nur Kontakte mit Ausländern gemeint, sondern auch mit Vertretern anderer Kulturen, die innerhalb der eigenen Gesellschaft leben. Kontakte zu solchen Personen können in Irkutsk nicht vermieden werden, weil viele Auswanderer aus ehemaligen Sowjetrepubliken und Vertreter von nördlichen asiatischen Völkern wie Burjaten, Jakuten, Tofen etc. in der Stadt leben. Einige Vertreter der oben erwähnten Völker waren auch Teilnehmer des Experiments.

Diese Frage spricht auch die Einstellungen der Teilnehmer an, da es hier um solche Kontakte wie Freundschaft geht, was ein emotionales Engagement der Kursanten für interkulturelle Kontakte erfordert. Von 30 Experimentteilnehmern haben 20 angegeben, einen fremdkulturellen Freund zu haben, unter diesen 20 sind auch die vier Personen, die schon Auslandserfahrungen hatten. 10 Kursanten haben die Frage negativ beantwortet. Wie sich der Kontakt zum fremdkulturellen Partner auf die Entwicklung der interkulturellen Kompetenz ausgewirkt hat, zeigt Abbildung 18. 
Abbildung 18: Prä-/Posttest-Vergleich: Veränderungen der interkulturellen Kompetenz in den EG nach Angaben über interkulturelle Freundschaften

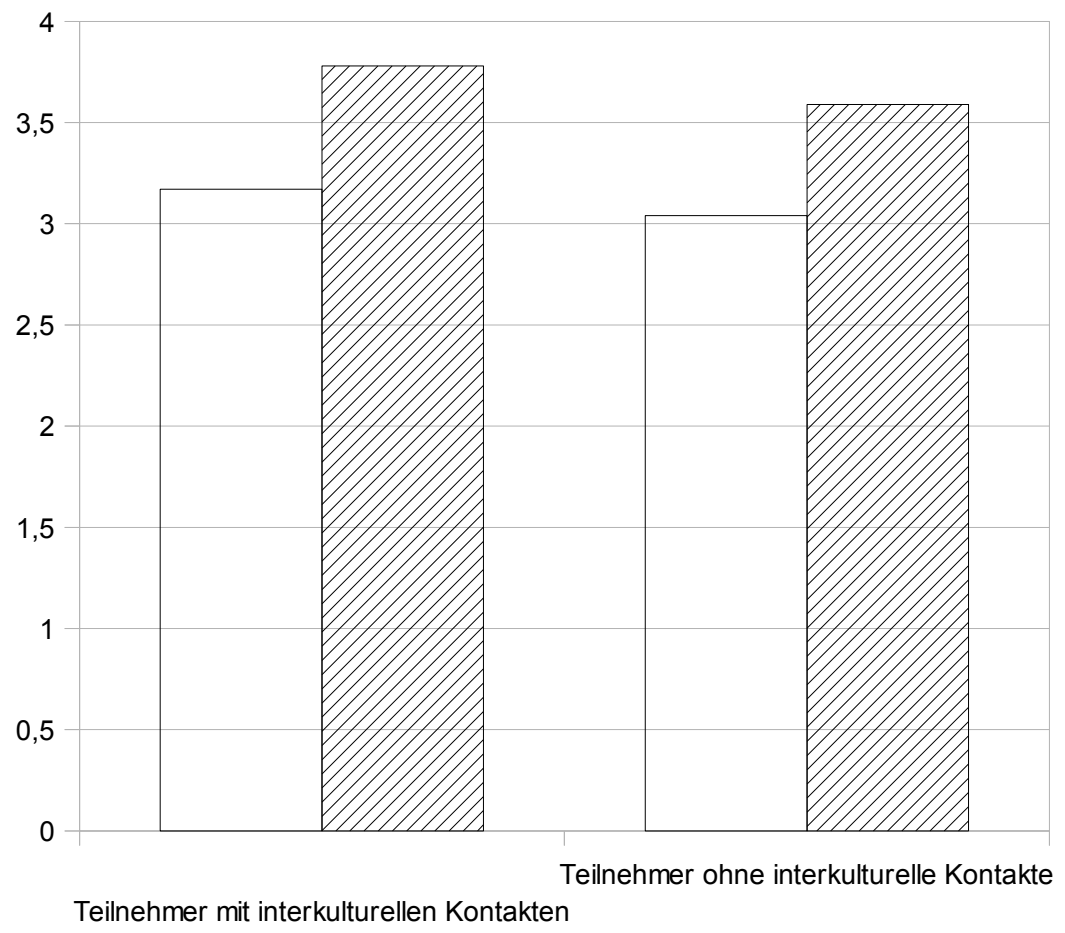

Wie aus der Abbildung ersichtlich, hat die Freundschaft mit einem Vertreter einer anderen Kultur keine Auswirkungen auf die Ergebnisse des Experiments. Beide Gruppen unterscheiden sich weder im Prä- noch im Posttest kaum voneinander. Im Prätest zeigen beide Gruppen ein relativ ähnliches Ergebnis (3,17 für die Personen mit interkulturellen Freundschaften und 3,04 für die Personen ohne solche Freundschaften). Zum Abschluss des Experiments zeigen die beiden Gruppen im Posttest ähnliche Veränderungen: 3,78 für die erste Gruppe und 3,59 für die zweite Gruppe. Zusammenfassend lässt sich sagen, dass der nähere Kontakt mit Vertretern einer anderen Kultur innerhalb der eigenen Gesellschaft keine Auswirkungen auf die Entwicklung der interkulturellen Kompetenz hat.

Zusammenfassung: In den Kapiteln 7.1 und 7.2 wurden die Ergebnisse des Prä- und Posttests präsentiert und analysiert. Es wurden Vergleiche zwischen den Daten der experimentellen Gruppen und den Kontrollgruppen gezogen mit dem Ergebnis, dass die Ersteren eine bessere Entwicklung der interkulturellen Kompetenz gezeigt haben als die Letzteren. Im Kapitel 7.3. wurde darauf eingegangen, wie sich die drei Bestandteile der interkulturellen Kompetenz (Kenntnisse, Sensibilität und Fähigkeiten) verändert haben, ebenso welche von 
den Teilkompetenzen eine höhere Stufe der Entwicklung erreicht haben und wo die Experimentteilnehmer Schwierigkeiten hatten. Zum Schluss wurden im Kapitel 7.4. die Erfolge der experimentellen Gruppen bezüglich unabhängiger Faktoren wie Gruppenzugehörigkeit, Geschlecht und interkulturelle Erfahrungen präsentiert und analysiert.

\subsection{Diskussion der Ergebnisse}

Ein Ziel der vorliegenden Dissertation war festzustellen, ob die Entwicklung der interkulturellen Kompetenz innerhalb des Fremdsprachenunterrichts mithilfe des von der Autorin ausgearbeiteten Programms (PEIK) möglich ist. Das im Rahmen der Untersuchung durchgeführte Experiment hat bewiesen, dass der Fremdsprachenunterricht durch PEIK ergänzt werden kann. Die erste Erprobung des Programms hat gezeigt, dass der interkulturelle Gedanke durch Arbeit an interkulturellen Missverständnissen, an der Selbstreflexion und an nationalen Stereotypen an die Sprachlernenden transportiert werden kann, was zur Entwicklung der interkulturellen Kompetenz beiträgt. Der Unterricht soll nicht nur die Vermittlung theoretischer Kenntnisse, sondern auch die praktische Auseinandersetzung mit interkulturellen Inhalten gewährleisten. Dazu wurden unterschiedliche Unterrichtsformen eingesetzt: Vorträge, Diskussionen, Spiele, Simulationen etc.

Die ersten Messungen haben gezeigt, dass das Niveau der interkulturellen Kompetenz in den Gruppen, die am Experiment teilgenommen haben, gestiegen ist. In den Kontrollgruppen, deren Fremdsprachenunterricht nach den üblichen Plänen ablief, wurden keine Veränderungen festgestellt. Dies bestätigt die Haupthypothese dieser Arbeit. Da es sich in diesem Fall um ein Feldexperiment handelt, das im Unterschied zu einem Laborexperiment unter natürlichen Bedingungen durchgeführt wurde, konnten die Wirkungen aller äußeren Einflüsse auf die Ergebnisse nicht gemessen werden. Die Begegnungen mit Vertretern anderer nationaler Kulturen oder die Unterrichtsmaterialien, die in anderen Fächern bearbeitet wurden, könnten zum Beispiel bestimmte Wirkungen auf die Entwicklung der interkulturellen Kompetenz ausgeübt haben.

Ein anderer Aspekt, der diese Ergebnisse in Frage stellen könnte, ist das Messinstrument selbst. Der von der Autorin auf der Grundlage von Fantinis „YOGA-Test“" ausgearbeitete Fragebogen stellt kein ideales Messinstrument dar. Genauso wie der Ursprungstest ist der Fragebogen nicht auf alle vier Gütekriterien geprüft.

In den Zielsetzungen des Experiments wurden noch zwei weitere Hypothesen aufgestellt. 
So wurde vermutet, dass eine der Teilkomponenten der interkulturellen Kompetenz, und zwar die Komponente „Kenntnisse“, sich besser entwickeln würde als die zwei anderen. Bei der ,interkulturellen Sensibilität“ und den ,interkulturellen Fähigkeiten“ waren zwar Veränderungen zu erwarten, das Maß dieser Veränderungen sollte aber geringer im Vergleich zu den Veränderungen im Bereich „Kenntnisse“ sein. Es wurde angenommen, dass die Entwicklung der affektiven Seite Zeit und die Entwicklung der Fähigkeiten Praxis braucht. Diese Hypothesen fanden keine Bestätigung durch das Experiment - im Gegenteil haben die Ergebnisse gezeigt, dass sich alle drei Komponenten der Kompetenz relativ gleichmäßig entwickelt haben. Im Unterricht wurde viel Wert darauf gelegt, das erlernte Wissen in die Praxis umzusetzen. Da authentische Kontakte fehlten (es wurden innerhalb des Experimentes keine Treffen mit fremdkulturellen Gesprächspartnern organisiert), wurde versucht, mithilfe von „Fremdheit darstellenden“ Lernsituationen und aufgrund der eigenen Erfahrungen der Teilnehmer die möglichen praktischen Potenziale auszunutzen und dadurch praktische Fähigkeiten zu entwickeln. Auch der Versuch, die Einstellungen der Teilnehmer zu ändern, scheint erfolgreich zu sein. Die interkulturelle Sensibilität erfuhr am wenigsten Veränderung im Vergleich zu den Kenntnissen und Fähigkeiten, diese Veränderungen waren aber dennoch bedeutsam.

Das Programm zur Entwicklung der interkulturellen Kompetenz beinhaltet vier Themen, und zwar kritische Ereignisse (interkulturellen Missverständnisse), Selbstreflexion, nationale Stereotype und Landeskunde. Im Fragebogen, der in der vorliegenden Arbeit benutzt wurde, wurden nur Fragen zu drei Themenbereichen gestellt. Fragen über nationale Stereotype wurden in allen drei Teilen des Fragebogens nicht gestellt. Die Kenntnisse der Teilnehmer über vorgefasste Meinungen, ihre Sensibilität und die Fähigkeit zum Umgang mit Stereotypen wurden im Puzzle-Fragebogen direkt nicht bewertet. Da aber die interkulturelle Kompetenz hier als ein komplexes Phänomen verstanden wurde, wurden einige Einstellungen und Fähigkeiten erfasst, die einen direkten Einfluss auf den Umgang der Teilnehmer mit dem Thema „Stereotype“ haben. So wurde zum Beispiel das Empathievermögen innerhalb der affektiven Einstellungen gemessen.

Bei der Beschäftigung mit kritischen Ereignissen wurden sowohl Kenntnisse über den Begriff „Kultur“ als auch über Kulturtheorien erworben, auf Grundlage dieser Kenntnisse wurden einige interkulturelle Missverständnisse analysiert und über eigene Erlebnisse reflektiert und diskutiert. Diese Übungen haben nach der Selbstbewertung der Teilnehmer Verbesserungen innerhalb aller drei Komponenten der interkulturellen Kompetenz erzielt. Die Teilnehmer konnten danach besser den Begriff „Kultur“ definieren, einige kulturelle 
Einstellungen und Werte nennen, aber auch ihr Einfluss auf die interkulturelle Kommunikation wurde den Teilnehmern bewusster. Auf dem Einstellungsniveau sind sowohl das gewachsene Interesse nicht nur für die Sprache, sondern auch für Verhaltensregeln, nonverbale Kommunikation und die Bereitschaft, auf den fremdkulturellen Partner einzugehen, zu beobachten. Die Kenntnisse und affektiven Einstellungen zeigen sich in der Fähigkeit zu Vergleich und Analyse der interkulturellen Situation, in der Fähigkeit, die Gründe für interkulturelle Missverständnisse zu erkennen und in den kulturellen Einstellungen und fremden Werten zu suchen, in der Fähigkeit, über die misslungene interkulturelle Kommunikation nachzudenken.

Es ist nicht ausgeschlossen, dass die eigenen Erfahrungen der Schüler und Schülerinnen innerhalb des experimentellen Zeitraums auch dazu beigetragen haben, einige der oben erwähnten Fähigkeiten und Einstellungen zu entwickeln. Solche Eigenschaften wie Empathievermögen oder Ambiguitätstoleranz, die für die interkulturelle Kompetenz von besonderer Bedeutung sind, könnten auch außerhalb des experimentellen Rahmens entwickelt werden, z.B. in Situationen in studentischen Wohnheimen oder in der Lerngruppe. Ebenso könnte die Fähigkeit zu Vergleich und Analyse allgemein auch in anderen Lernfächern entwickelt worden sein. Die Experimentteilnehmer könnten auch misslungene Kommunikationen innerhalb der eigenen Kultur erlebt und darüber nachgedacht haben, was sicherlich auch einen Einfluss auf die positive Entwicklung ihrer Fähigkeiten hinterlassen hat. Auch wenn andere Faktoren für den Lernerfolg mitverantwortlich sind, wurden die Veränderungen mittels der Programminhalte auf den ,interkulturellen“ Punkt gebracht, d.h. für die Situationen des interkulturellen Kontakts spezifiziert.

Einen weiteren Beitrag zur Sensibilisierung gegenüber kulturellen Unterschieden und zur Entwicklung der Fähigkeiten im Umgang mit interkulturellen Situationen sollte die Arbeit mit landeskundlichen und sprachlichen Übungen liefern. Sie bilden den vierten Teil des PEIK. Diese traditionellen Inhalte des Fremdsprachenunterrichts sind unter dem neuen interkulturellen Blickwinkel zu sehen. Es geht dabei um Kenntnisse über die Kultur, deren Sprache erlernt wird, im Fall des Experiments um die deutsche Kultur. Sie wurden im Fragebogen nicht direkt erfasst, stattdessen wurden Veränderungen in den Einstellungen und den entsprechenden Fähigkeiten diagnostiziert. Die neue Akzentuierung, in diesem Fall die interkulturellen Aspekte der landeskundlichen und sprachlichen Informationen, die die Einbeziehung der Informationen über die eigene Kultur erfordern, scheint positiv auf die Motivation der Teilnehmer zum Erlernen der Fremdsprache gewirkt zu haben.. Die Teilnehmer der experimentellen Gruppen berichteten über ein gewachsenes Interesses zum Erlernen 
einer Fremdsprache und ihrer Kultur. Dabei bestätigten sie auch die Entwicklung der eigenen Fähigkeiten, diese Kenntnisse verbessern zu können.

Die These, dass sich die Einführung von interkulturellen Inhalten positiv auf die Motivation zum Erlernen einer Fremdsprache auswirkt, konnte nur aufgrund der indirekten Indizien aufgestellt werden. Da es nicht in den Aufgabenbereich des Fragebogens fiel, wurde die Motivation der Teilnehmer vor und nach dem Experiment nicht gemessen.

Am schwersten fiel den Teilnehmern der Umgang mit der Selbstreflexion. Obwohl mehrere Übungen dazu sowohl in der theoretischen als auch der praktischen Ausrichtung im Programm eingeschlossen waren, waren die Erfolge der Teilnehmer in diesem Bereich nicht eindeutig. Auf der einen Seite gaben die Teilnehmer in allen drei Verhaltensbereichen (Kenntnisse, Sensibilität und Fähigkeiten) an, dass sie interkulturelle Begegnungen als eine Situation betrachten, die durch beide Gesprächspartner kulturell geprägt ist. Das drückt sich in der Bereitschaft aus, über die eigenen kulturellen Einstellungen nachzudenken und sie während der interkulturellen Kommunikation in den Hintergrund zu rücken, und in der Überzeugung, passende Strategien bei misslungener Kommunikation finden zu können. Dies erfordert einen gewissen Grad an Selbstreflexion.

Auf der anderen Seite, scheiterte die Selbstreflexion, wenn es um Emotionen geht. Schon am Anfang des Experiments zeigten die Teilnehmer sowohl auf dem Bewusstseins- als auch auf dem Einstellungsniveau geringe Kompetenzen im Umgang mit Emotionen. Die Ergebnisse des Fragebogens zeigten, dass sie auf die mögliche Existenz negativer Emotionen nicht eingegangen sind. Im Grunde genommen sind die Teilnehmer davon überzeugt, dass sie emotionsmäßig jede Situation des interkulturellen Kontakts bewältigen können oder bei einem Fehler durch unterschiedliche Strategien diese Situation korrigieren können. Es zeigte sich, dass sie die Rolle der Emotionen, sowohl der fremden aber auch der eigenen, in realen Kommunikationssituation unterschätzen.

An dieser Stelle ist zu unterstreichen, dass gerade die kulturelle Selbstreflexion eine der schwersten Aufgaben ist, die ein Teilnehmer der interkulturellen Kommunikation zu lösen hat. Es ist zwar möglich, innerhalb des Lernprogramms darauf hinaufzuweisen, darüber zu sprechen und die vorliegenden Situationen aus dem eigenen kulturellen Gesichtspunkt zu analysieren. In der realen Situation des interkulturellen Kontaktes ist das nicht immer möglich. Nicht alles kann mit dem fremdkulturellen Partner besprochen werden, es werden immer Lücken gelassen, deren Gründe durch Selbstreflexionen vermutet werden können, aber Sicherheit und Antworten gibt es nicht immer. Emotionen spielen dabei keine geringe Rolle. Der Kommunikationsteilnehmer mag die besten Absichten gegenüber dem fremdkultu- 
rellen Partner haben, wenn er aber auf dem emotionalen Niveau betroffen ist, werden sämtliche Einstellungen und Fähigkeiten für eine Weile blockiert. Deshalb ist es wichtig für den Teilnehmer zu begreifen, dass eine Selbstreflexionen im affektiven Bereich genauso bedeutungsvoll ist wie die kulturelle, aber auch sie kann keine Erfolgsgarantie im Fall der interkulturellen Kommunikation sein. Dabei wären eigene interkulturelle Erfahrungen der Teilnehmer von Bedeutung, besonders solche, die mit Misserfolgen verbunden waren.

Die Frage, ob und wie die eigene Erfahrung im interkulturellen Bereich die Entwicklung der interkulturellen Kompetenz beeinflusst, wurde in diesem Experiment nicht gründlich erforscht. Jedoch lassen die Ergebnisse der Untersuchung folgende These zu: Erfahrungen im Bereich der interkulturellen Kommunikation wie ein kurzfristiger Auslandsaufenthalt oder die Existenz fremdkultureller Freundschaften machen den Menschen nicht automatisch interkulturell kompetent. Unter dem Einfluss der interkulturellen Lerninhalte haben aber solche Menschen bessere Entwicklungsmöglichkeiten.

Es lässt sich vermuten, dass Menschen mit interkulturellen Erfahrungen ein inneres Potenzial haben, worauf sie bei der Aufnahme kulturübergreifender Informationen und bei interkulturell orientierten Übungen Zugriff haben. Solche Menschen scheinen ihre Sensibilität besser entwickeln zu können als diejenigen, die keine interkulturelle Erfahrung haben. Fazit: Interkulturelle Kontakte zu erleben ist etwas anderes, als darüber auf Grundlage von theoretischen Kenntnissen nachzudenken.

An diesem Punkt wäre die Frage angebracht, wie positiv oder negativ die interkulturellen Erfahrungen der Teilnehmer gewesen sind. Wenn die Ergebnisse bezüglich des Umgangs mit Emotionen in interkulturellen Situationen betrachtet werden, lässt sich vermuten, dass die Teilnehmer des Experiments überwiegend positive Erfahrungen gemacht haben. Unter der Wirkung des interkulturellen Lernens entwickeln sich positive Voraussetzungen, indem solche Einstellungen wie Empathie, Offenheit und Toleranz verbessert werden. Da die Teilnehmer selbst wahrscheinlich keine negativen Erfahrungen in der Situation des interkulturellen Kontakts gemacht haben, wurden die Informationen über die negativen Emotionen bezüglich des fremdkulturellen Partners von den interkulturellen Lerninhalten nicht beeinflusst und nicht verarbeitet.

Eine weitere These könnte nach Ansicht der Verfasserin nach der Analyse der Ergebnisse aufgestellt werden. Es lässt sich vermuten, dass die Entwicklung der interkulturellen Kompetenz von den Lernerfolgen der Teilnehmer abhängig ist. Diejenigen, die bessere Noten im Fach „Fremdsprache“ haben, zeigen auch eine bessere Entwicklung der interkulturellen Kompetenz. Sie übertragen quasi ihre Lernerfolge auch in den interkulturellen Bereich. 
Diese Annahme kann durch Folgendes erklärt werden: Das Puzzle-Modell wird in dieser Arbeit als Lernmodell betrachtet, also die interkulturellen Informationen und Übungen sollten dazu beitragen, bestimmte Kenntnisse und Fähigkeiten zu entwickeln. Der Teilnehmer, der durch eigenen Lernwillen und gute Voraussetzungen beim Erlernen einer Fremdsprache gut vorankommt, ist auch bei der Entwicklung der interkulturellen Kompetenz erfolgreich.

Alle Thesen, die in der oben angeführten Diskussion aufgestellt und besprochen wurden, sind nur mit Vorbehalt zu verallgemeinern. Der Hauptgrund ist die geringe Zahl der Probanden, die an dem Experiment teilgenommen haben.

Das Programm zur Entwicklung der interkulturellen Kompetenz wurde zum ersten Mal erprobt und in einem Experiment evaluiert. Die Ergebnisse der Messungen bestanden den Signifikanztest, was zur weiteren Arbeit mit diesem Programm ermutigt. Das Hauptziel der vorliegenden Arbeit bestand jedoch nicht darin, die Wirksamkeit von PEIK auf die Entwicklung der interkulturellen Kompetenz der Sprachlernenden zu beweisen. Viel mehr Wert wurde darauf gelegt zu überprüfen, ob PEIK durchführbar ist, ob es von den Studierenden angenommen wird und Lehrkräfte damit arbeiten können. In der weiteren Perspektive ist die Anwendung des Programms in weiteren Gruppen von der Verfasserin vorgesehen, was logischerweise zu einer größere Anzahl an Probanden führt.

\section{Zusammenfassung und Ausblick}

Die Entwicklung der interkulturellen Kompetenz gehört zukünftig zur Aufgabe jeder Bildungseinrichtung, vom Kindergarten bis zur Hochschule. Neben der Gewährleistung der Bürgerrechte und Verabschiedung der Gesetze gegen Rassismus muss die Entwicklung der interkulturellen Kompetenz auch als politische Aufgabe verstanden werden. Das verlangt sowohl Unterstützung öffentlicher Initiativen und Maßnahmen als auch entsprechende Projekte in der interkulturellen Marktwirtschaft. Interkulturelle Kompetenz muss als Schlüsselqualifikation in den Anforderungsprofilen nicht nur für international tätige Mitarbeiter, sondern auch für die Mitarbeiter sozialer Einrichtungen wie Krankenhäuser, soziale Dienste, Verwaltung gefordert werden.

Der Entwicklung interkultureller Kompetenz sollte in Ausbildungsstätten und Lerneinrichtungen große Bedeutung beigemessen werden. In Kindergärten, Schulen, Fach- und Hochschulen, Universitäten etc. ist eine Atmosphäre notwendig, in der Kinder und Jugendliche 
gefördert werden, um die Natur und die Bedeutung der Globalisierung zu verstehen und Fähigkeiten zur effektiven Kommunikation mit Vertretern anderer kultureller Kreise zu entwickeln. Zudem trägt die interkulturelle Kompetenz viel zur weiteren Lebensplanung der Kinder und Jugendlichen bei, da ihre Berufschancen durch ihre sozialen Kompetenzen steigen.

Da die Bedeutung der interkulturelle Kompetenz in der modernen Gesellschaft immer mehr steigt, steht sie zur Zeit im Mittelpunkt wissenschaftlichen Interesses. Neben vielen praktischen Projekten und Ausarbeitungen ist eine Zunahme unterschiedlicher theoretischer Konzepte zu beobachten. Die meisten Arbeiten werden entweder im Bereich des interkulturellen Managements oder in der interkulturellen Pädagogik zu den Problemen der multikulturellen Gesellschaft veröffentlicht. Zunehmende Bedeutung gewinnt jedoch das angesprochene Konzept im Fremdsprachenunterricht. Dabei geht es nicht nur um den Unterricht innerhalb des Landes der zu erlernenden Sprache (z.B. um Deutsch als Zweitsprache in Deutschland), sondern auch um den Unterricht im Ausland (z.B. Deutsch als Fremdsprache in Russland). Wie sich die Zielvorstellungen über den Fremdsprachenunterricht entwickelt haben, wird in der vorliegenden n Arbeit erläutert und diskutiert.

Theoretische Erklärungsansätze wie die von Fischer (2005), Fantini (2000) und Byram (1997) stellen unterschiedliche Sichtweisen auf das umstrittene und vielseitige Konzept ,interkulturelle Kompetenz' dar. Die Verfasserin diskutiert diese Theorien, entwickelt in der vorliegenden Arbeit jedoch ein eigenes Modell, das in der weiteren Arbeit als PuzzleModell bezeichnet wird. Die Besonderheit des Modells besteht darin, dass es auf die Entwicklung der interkulturellen Kompetenz im Sprachlernprozess abgestimmt ist. Die Verfasserin sieht die angesprochene Kompetenz als eine Art Puzzle, dessen drei Grundbestandteile Kenntnisse, Sensibilität und Fähigkeiten sind. Zusätzlich wird die interkulturelle Kompetenz durch andere Kompetenzen, Fähigkeiten und Einstellungen wie durch PuzzleStückchen an der Peripherie ergänzt.

In der Arbeit werden die einzelnen Bestandteile des Puzzle-Modells präsentiert und Beispiele aus dem Fremdsprachenunterricht angeführt. Die erste Komponente betrifft die kognitive Seite, kulturspezifische und kulturübergreifende Kenntnisse. Die zweite betrifft die affektive Seite der Kompetenz, die Einstellung der Lernenden zur Interkulturalität. Sie wird im Puzzle-Modell durch interkulturelle Sensibilität präsentiert, die sich zeigt in Empathie und der Bereitschaft, die eigenen kulturellen Normen zu hinterfragen und zu relativieren. Weitere Aspekte der interkulturellen Sensibilität sind nach Auffassung der Verfasserin Offenheit, Respekt und Ambiguitätstoleranz. Auf der Ebene des Verhaltens, der dritten 
Komponente, wird die interkulturelle Kompetenz durch interkulturelle Fähigkeiten präsentiert, die bei den Lernenden Voraussetzungen dazu schaffen, auf Grundlage der Kenntnisse und mit Hilfe der interkulturellen Sensibilität in den Situationen interkultureller Kontakte adäquat und erfolgreich zu kommunizieren.

Die wenigsten in der Literatur zu findenden Konzepte der interkulturellen Kompetenz geben eine Anleitung zur praktischen Umsetzung. Dies gilt auch in Bezug auf den Fremdsprachenunterricht: Obwohl interkulturelle Kompetenz als ein Ziel des Unterrichts erklärt wird, fehlen Programme zur Umsetzung in der Praxis. Dabei bieten die interkulturellen Trainings, die für das interkulturelle Management ausgearbeitet worden sind, gute Materialien und Ideen. In dem von der Verfasserin ausgearbeiteten Programm zur Entwicklung der interkulturellen Kompetenz (PEIK), das sich am Puzzle-Modell orientiert, wird versucht, das Gedankengut der interkulturellen Didaktik zugunsten der Aufgaben des Fremdsprachenunterricht zu nutzen.

Um zu überprüfen, ob PEIK durchführbar ist und ob die Lehrkräfte damit in Zukunft arbeiten können, wurde das Programm im Rahmen eines Experimentes in dem Ost-Sibirischen Institut des Ministeriums des Inneren (Irkutsk, Russland) eingesetzt. Vor der Einführung und nach dem Abschluss des Programms wurde das Niveau der interkulturellen Kompetenz sowohl in den experimentellen als auch in Kontrollgruppen gemessen. Dazu wurde von der Verfasserin der Puzzle-Fragebogen entwickelt, der auf dem Puzzle-Modell beruht ist.

Das wichtigste Ergebnis ist die Feststellung, dass das Programm gut in den Fremdsprachenunterricht integriert werden kann. Die interkulturellen Inhalte des Programms ergänzen die traditionelle Sprachvermittlung und werden von den Lernenden angenommen.

Die erste Erprobung hat auch gezeigt, dass das Niveau der interkulturellen Kompetenz in den experimentellen Gruppen deutlich gewachsen ist im Vergleich zu dem der Kontrollgruppen. Dabei betreffen die positiven Veränderungen alle drei Komponenten des PuzzleModells, d.h. Kenntnisse, Sensibilität und Fähigkeiten. Bei der Evaluation wurden die einzelnen Fragen ausgewertet, was ermöglichte, die Schwierigkeiten und die Erfolge beim Einsatz des Programms herauszufinden. Es wurde auch analysiert, welchen Einfluss unabhängige Faktoren wie Gruppenzugehörigkeit, Geschlecht und interkulturelle Erfahrungen auf die Entwicklung der interkulturellen Kompetenz haben.

Die Unterschiede zwischen den Prä- und den Posttests erwiesen sich als signifikant, aber ihre Aussagekraft darf aufgrund der kleinen Stichprobe nicht überbewertet werden. Die Ergebnisse ermutigen jedoch dazu, mit dem Programm weiterzuarbeiten, um es anhand grö- 
Berer Stichproben zu evaluieren.

Die vorliegende Arbeit stellt einen wichtigen Schritt zur Erforschung der Thematik des interkulturellen Lernens dar. Die Verfasserin betrachtet ihre Arbeit an dieser Problematik jedoch nicht als abgeschlossen. Deshalb soll zunächst kritisch angemerkt werden, welche Grenzen bzw. Probleme sich bei der Arbeit gezeigt haben, um daraus Schlussfolgerungen für weitere Forschungen zu diesem Thema zu ziehen.

Im Folgenden werden die Grenzen der vorliegenden Arbeit aufgezeigt:

1. Die erste Erprobung des Programms zur Entwicklung interkultureller Kompetenz (PEIK) hat gezeigt, dass das Programm gut einsetzbar ist und die angestrebten Ziele im Fremdsprachenunterricht damit erreicht werden können. Die Verfasserin selbst hat innerhalb eines Semesters als Lehrkraft in zwei Gruppen das Experiment durchgeführt. Obwohl die Effektivität experimentell in diesen Gruppe nachgewiesen werden konnte, können die Ergebnisse nicht verallgemeinert werden.

2. Das Programm zur Entwicklung der interkulturellen Kompetenz (PEIK) wird in dieser Arbeit lediglich in Auszügen präsentiert. Ihre Aufgabe sah die Verfasserin darin, einen Überblick über die Struktur und den Aufbau des Programms zu geben, weshalb lediglich Beispiele aus PEIK zur Veranschaulichung dargestellt werden. Während der ersten Erprobung wurden auch „Schwachstellen“ des Programms, wie z.B. Probleme bei der Übersetzung der Programminhalten ins Russische oder bei der Formulierung der Diskussionsfragen, entdeckt, die der weiteren Überarbeitung bedürfen.

3. Ein weiterer Kritikpunkt betrifft den Puzzle-Fragebogen, der für die Messung der interkulturellen Kompetenz eingesetzt wurde. Es ist notwendig, die einzelnen Fragen zukünftig auf ihre Tauglichkeit zu überprüfen und das Instrument auf Gütekriterien zu untersuchen.

4. Obwohl die Messungen nach dem Einsatz des Programms einen Fortschritt in der Entwicklung der interkulturellen Kompetenz gezeigt haben, wurden sie nicht als Hauptziel der vorliegenden Arbeit betrachtet. Viel mehr Wert wurde darauf gelegt zu überprüfen, ob das Programm durchführbar ist, und ob sie von Lehrkräfte und Studenten angenommen wird.

Die vorliegenden Ergebnisse ermutigen dazu, weiter an dem Programm zu arbeiten. Die Korrektur der Programminhalte und die Überarbeitung seiner „Schwachstellen“ ist vorgesehen. Anschließend ist die Veröffentlichung von PEIK geplant.

- Nach der Veröffentlichung von PEIK ist sein weiterer Einsatz im Fremdsprachen- 
unterricht vorgesehen. Die Veröffentlichung des Programms ermöglicht die Einbeziehung weiterer Lehrkräfte, die nach der Bekanntmachung mit den Zielen von PEIK diese mithilfe des angebotenen Materials in ihrem Unterricht verwirklichen können.

- Damit die Ergebnisse evaluiert werden können, muss die Arbeit an dem Messinstrument fortgesetzt werden. Der Puzzle-Fragebogen kann als Grundlage dafür verwendet werden.

- Nach der Veröffentlichung von PEIK und der Verbesserung des Puzzle-Fragebogens ist es möglich, im Rahmen eines Projekts weitere Stichproben durchzuführen. Diese Weiterentwicklung von Unterrichtsprogramm und Messinstrument erlauben im nächsten Schritt, die Tauglichkeit des PEIK für die Entwicklung der interkulturellen Kompetenz nachzuweisen.

Zusammenfassend kann festgestellt werden, dass in der vorliegenden Arbeit die interkulturelle Kompetenz unter pädagogischem Gesichtspunkt betrachtet wurde. Sie ist aber auch Gegenstand fachlicher Diskussionen in anderen Wissensgebieten und wird mittlerweile als eine Schlüsselkompetenz sowohl für die meisten Fachkräfte als auch für jedes Mitglied einer multikulturellen Gesellschaft betrachtet. Umstritten sind jedoch die Konzepte in den unterschiedlichen Wissensgebieten, d.h. dass ein theoretisches Konzept zur interkulturellen Kompetenz noch aussteht. Es wäre zu wünschen, dass aus einem Schlagwort, zu dem „interkulturelle Kompetenz" geworden ist, ein wissenschaftlich begründetes vereinheitlichtes Konzept entsteht, dessen inhaltliche Bedeutung und Dimensionen für alle gültig und verständlich sein sollten.

Die überwiegende Zahl der praktischen Ausarbeitungen und Veröffentlichungen zu dem angesprochenen Thema beschäftigt sich mit der interkulturellen Kompetenz im binationalen Bereich. Es handelt sich vorwiegend um Programme und Projekte im interkulturellen Management, die die Mitarbeiter eines international tätigen Unternehmens für den Aufenthalt in einer fremden Kultur vorbereiten. Die übliche, relativ einfache Methode ist, die Unterschiede zwischen nur zwei Kulturen hervorzuheben. Dieses Konzept ist bezüglich der multikulturellen Gesellschaft nicht tauglich. Mit der Vereinheitlichung des gesamten praktischen Konzepts können die vielfältigen zur Zeit laufenden Projekte dann unter einem Dach vereint werden. Die einheitliche Linie kann m.E mehr zur Verwirklichung des Ziels „,interkulturelle Kompetenz“ beitragen.

Ein einheitliches Konzept der interkulturellen Kompetenz wird letztendlich auch die umstrittene Frage des Messverfahrens lösen. Die zur Zeit existierenden Messinstrumente rich- 
ten sich immer an einem bestimmten Modell aus, das, wie oben schon erwähnt, meistens einseitig ist, und diese werden logischerweise von den Vertretern anderer Modelle als mangelhaft kritisiert. Die Schlüsselkompetenz des 21. Jahrhundert braucht m.E. ihr eigenes Messverfahren, das nicht nur auf die Selbsteinschätzung des Probanden zurückgreift, sondern auch die Bewertung anderer Personen einbezieht.

Obwohl das Konzept der interkulturellen Kompetenz noch viele Mängel und Lücken aufweist, ist seine Bedeutung unbestritten. Um weiter in der modernen Gesellschaft, die immer reicher an unterschiedlichen Nationalitäten und Kulturen wird, zu leben, wird interkulturelle Kompetenz gebraucht. Hier ist an die Metapher vom „Raumschiff Erde“ des amerikanischen Ökonomen Boulding (1966) zu erinnern. Um in einem geschlossenen Raum überleben zu können, muss die Besatzung des Schiffes nicht nur an den eigenen Profit denken, sondern die Probleme gemeinsam lösen. Das ist aber nur unter solchen Bedingungen wie Solidarität, Akzeptanz und Zusammenhalt möglich. Die Situation betrifft auch alle Mitglieder der modernen Gesellschaft. Die Fähigkeit, miteinander adäquat kommunizieren zu können, was unter den Bedingungen der modernen Gesellschaft interkulturelle Kompetenz voraussetzt, ist die Überlebenschance für ihre Mitglieder. (vgl. Bühler 1996, S. 62) 


\section{Literaturverzeichnis}

Abendroth-Timmer, D. (1997). Zum Potenzial von Lehrwerken für das Verstehen anderer Kulturen. In L. Bredella, H. Christ, M. K. Legutke (Hrsg.), Thema Fremdverstehen. Arbeiten aus dem Graduiertenkolleg „Didaktik des Fremdverstehens“. Tübingen: Günter Narr, S. 76-100.

Abendroth-Timmer, D. (1999). Interkulturelles Lernen und das Bedingungsgefüge der Lehrwerkerstellung. In W. Börner, K. Vogel (Hrsg.), Lehrwerke im Fremdsprachenunterricht: Lernbezogene, interkulturelle und mediale Aspekte. Bochum: AKS-Verlag, S. 166190.

Anderson, L.W., Krathwohl, D.R. (Hrsg.)(2001). A Taxonomie of Learning, Teaching and Assessing: A Revision of Bloom's Taxonomie of Educational Objectives. Boston: Allyn \&Bacon.

Anikin, W. (1966). Vorwort zum Wörterbuch der russischen Sprichwörter. Original: Аникин, В.П. (1966). Предисловие к книге: Жуков В.П. Словарь русских пословиц и поговорок. Москва.

Antor, H. (2002). Die Vermittlung interkultureller Kompetenz an der Universität. Das Beispiel Kanada. In L. Volkmann, K. Stierstorfer, W. Gehring (Hrsg.), Interkulturelle Kompetenz. Konzepte und Praxis des Unterrichts. Tübingen: Günter Narr, S. 143-163.

Arbeitskreis Staatsinstitut für Schulpädagogik und Bildungsforschung (1997). Die Münchner Thesen zum interkulturellen Lernen. In LIFE. Ideen und Materialien für interkulturelles Lernen. München: BMW-Group.

Auernheimer, G. (2002). Interkulturelle Kompetenz - ein neues Element pädagogischer Professionalität? In Auernheimer (Hrsg.). Interkulturelle Kompetenz und pädagogische Professionalität. Opladen: Leske\&Budrich, S. 183-205.

Auernheimer, G. (2003). Einführung in die interkulturelle Pädagogik. Darmstadt: Wissenschaftliche Buchgesellschaft.

Auernheimer, G. (2005). Interkulturelle Kommunikation und Kompetenz. In „Migration und soziale Arbeit", Heft 1, S. 15-22. 
Aufderstaße, H., Bock, H., Müller, J., Müller H. (2000). Themen neu 2. Lehrwerk für Deutsch als Fremdsprache. Ismaning: Max Hueber.

Batik, G. (1993), Bewusstsein und Materie oder Kräfte, die das Universum gestalten. Focus Newsletter, Nr. 1/1993. Letzter Zugriff am 12.12.2010 unter: http://www.focus.at/archiv/gba_bum1.htm.

Baxter, J. (1983). English for Intercultural Competence. In D. Landis, R. W. Brislin (Hrsg.), Handbook of Intercultural Training, Vol. 2. New York: Pergamon, S. 290-324.

Behnken, I., Brücker, H., Hölscher, P., Grummes-Unruh, S., Ilmberger A., Karaduman M., Stürme, M., Wolframm, S. (1997). Quartetto. Ein Kartenspiel mit Tieren und Tierlauten in verschiedenen Sprachen. In LIFE. Ideen und Materialien für interkulturelles Lernen. München: BMW-Group.

Bennett, M. J. (1993). Towards ethnorelativism: a development model of intercultural sensitivity. In Paige R. M. (Hrsg.), Education for the intercultural experience. Yarmouth: Intercultural Press, S. 21-72.

Bildungsministerium der Russischen Föderation (2001a). Der staatliche Bildungsstandard für die höhere Berufsausbildung. Fachrichtung 023100. Original: Министерство образования РФ (2001а). Государственный образовательный стандарт высшего профессионального образования (временные требования у минимуму содержания и уровню подготовки выпускников). Специльность 023100, Правоохранительная деятельность. Квалификация - юрист. 8. февраляя 2001. Москва: Министерство образования РФ.

Bildungsministerium der Russischen Föderation (2001b). Der staatliche Bildungsstandard für die mittlere Berufsausbildung. Fachrichtung 0203. Original: Министерство образования РФ (2001b). Государственный образовательный стандарт среднего профессионального образования. Государственне требования к минимуму содержания и уровню подготовки выпускников по специальности 0203 Правоохранительная деательность. Квалификация — юрист. Москва: Министерство образования РФ.

Bittner, A. (o.J.): Interkulturelle Trainingsmethoden. Letzter Zugriff am 28.11.2010 unter: http://www.ifim.de/reports/methoden.pdf 
Bloom, B. (Hrsg.) (1972). Taxonomie von Lernzielen im kognitiven Bereich. 4. Auflage. Weinheim, Basel: Beltz.

Böhm, W. (2005). Wörterbuch der Pädagogik. 16 vollständig überarbeitete Auflage. Stuttgart: Alfred Kröner.

Bolten, J. (2007). Einführung in die Interkulturelle Wirtschaftskommunikation. Göttingen: Vandenhoeck\& Ruprecht.

Bolton, J. (2007). Einführung in die interkulturelle Wirtschaftkommunikation. Göttingen: Vandenhoeck\& Ruprecht.

Bortz, J., Döring N. (2006). Forschungsmethoden und Evaluation für Human- und Sozialwissenschaftler (4. Auflage). Berlin: Springer.

Bosse, E. (2003). Interkulturelles Training als Ergänzung universitärer Fremdsprachenkurse? In Eckerth, Johannes, Wendt, Michael (Hrsg.) Interkulturelles und transkulturelles Lernen im Fremdsprachenunterricht. Frankfurt/M.: Lang, S. 155-168.

Böttcher, M., Repper, D., Schöne, A. \& Stander J. (2003). Mit Fremdheitserfahrungen umgehen lernen. Simulation. Autobahn. Letzter Zugriff am 26.06.2007 unter http://www.ikkompetenz.thueringen.de/downloads/autobahn.pdf

Bredella, L. (1990). Das Verstehen literarischer Texte im Fremdsprachenunterricht“. Die Neueren Sprachen, 1990 (6), S. 562-583.

Bredella, L. (1994). Interkulturelles Verstehen zwischen Objektivismus und Relativismus. In K.-R. Bausch, H. Christ, H.-J. Krumm (Hrsg.), Interkulturelles Lernen im Fremdsprachenunterricht. Arbeitspapiere der 14. Frühjahreskonferenz zur Erforschung des Fremdsprachenunterrichts. Tübingen: Günter Narr, S. 21-30.

Bredella, L. (1995). Verstehen und Verständigung als Grundbegriffe und Zielstellungen des Fremdsprachenlehrens und -lernens? In L. Brandella (Hrsg.), Verstehen und Verständigung durch Sprachlernen? Dokumentation des 15. Kongresses für Fremdsprachendidaktik, veranstaltet von der Deutschen Gesellschaft für Fremdsprachenforschung, Gießen, 4.-6. Oktober 1993 Bochum: Universitätsverlag, S. 1-34.

Brislin, R., Yoshida T. (1994). Intercultural Communication Training: an Introduction. 
London/ New Delhi: Sage Publications.

Brockhaus (1996): Die Enzyklopädie. In 24 Bänden. 20., überarbeitete und aktualisierte Auflage. Leipzig, Mannheim: F. A. Brockhaus.

Bühler, H. (1996). Perspektivenwechsel?: unterwegs ui „globalen Lernen“. Frankfurt a. Main: IKO.

Buttjes, D. (1991). Culture in German foreign language teaching: making use of an ambiguous past. In D. Buttjes, M. Byram (Hrsg.), Mediating Languages and Cultures: Towards an Intercultural Theory of Foreign Language Education. Clevedon, Philadelphia: multilingual Matters LTD, S. 47-62.

Buttjes, D. (1991). Mediating languages and cultures: the social and intercultural dimension restored. In Buttjes, Byram (Hrsg.), a.a.O., S. 3-17.

Buttjes, D. (1995). Landeskunde-Didaktik und landeskundliches Curriculum. In K.-R. Bausch, H. Christ, H.-J. Krumm (Hrsg.), Handbuch Fremdsprachenunterricht. 3. Auflage Tübingen, Basel: Francke, S. 142-149.

Byram, M. (1997). Teaching and Assessing Intercultural Communicative Competence. Clevedon: Multilingual Matters.

Canale, M. (1983). From communicative competence to communicative language gedagogy. In Richards J., Schmidt, R. W. (Hrsg.), Language and communication. New York: Longman.

Canale, M., Swain, M. (1980). Theoretical bases of communicative approaches to second language learning and testing. In Applied Linguistics 1/1, S. 1-47.

Caspari, D. (1997). Lernziel ,interkulturelles Lernen/Fremdverstehen“: Was Fremdsprachenlehrer und -lehrerinnen darüber denken - drei Fallbeispiele. In Bredella, Christ, Legutke (Hrsg.), a.a.O., S. 55-75.

Cockwell, A. (2010). Entwicklung interkultureller Kompetenz von Fach- und Führungskräften durch Training und Coaching. Norderstedt: Books on Demand.

Deardorff. D. (o.J.). Interkulturelle Kompetenz - Schlüsselkompetenz des 21. Jahrhun- 
derts? Thesenpapier der Bertelsmann Stiftung auf Basis der Interkulturellen-KompetenzModelle von Dr. Darla K. Deardorff. Letzter Zugriff am 12.03.2007 unter: http://www.bertelsmann-stiftung.de/bst/de/media/xcms_bst_dms_17145_17146_2.pdf

Eckerth, E., Wendt, M. (2003). Brauchen wir einen inter- und/oder transkulturellen Fremdsprachenunterricht? In J. Eckerth, M. Wendt (Hrsg.), Interkulturelles und transkulturelles Lernen im Fremdsprachenunterricht. Frankfurt am Main u.a.: Lang.

Einhoff, J. (1993). Der interkulturelle Ansatz - Denkanstoß für die Textaufgabe? Praxis des neusprachlichen Unterrichts, 1993 (3), S. 248-256.

Einhoff, J. (2003). Multi-Kulti. Didaktische Ansätze, Zielvorstellungen und Themen im Englischunterricht des 21. Jahrhunderts. Fremdsprachenunterricht, Sonderheft Praxis 2003.

Fantini, A. (2005). „About Intercultural Communicative Competence: A Construct. Letzter Zugriff am 13.03.2011 unter http://www.sit.edu/SITOccasionalPapers/feil_appendix_e.pdf.

Fantini, A. E. (2000). A Central Concern: Developing Intercultural Competence. In SIT Occasional Papers Series. Brattleboro: VT.

Fischer, V. (2005). Interkulturelle Kompetenz - ein neues Anforderungsprofil für die pädagogische Profession. In V. Fischer, M. Springer, I. Zacharaki (Hrsg), Interkulturelle Kompetenz. Fortbildung - Transfer - Organisationsentwicklung. Schwalbach: Wochenschau, S. $33-47$.

Fischer, V. (o.J.). „Interkulturelle Kompetenz“ in der fachwissenschaftlichen Diskussion. Letzter Zugriff am 12.03.2007 unter http://www.iizdvv.de/deutsch/aktuelles/erfurtliteratur/Fischer.htm.

Flechsig, K.-H. (1995). Kleines Handbuch Didaktischer Modelle. Eichenzell: Verlag für lebendiges Lernen.

Flechsig, K.-H. (2001). Beiträge zum interkulturellen Training. Göttingen: Institut für interkulturelle Didaktik.

Focus online (2007). Marco W. Wollte Sex mit Engländerin. Interview von 26.06.2007. Letzter Zugriff am $\quad 1.12 .2010$ unter http://www.focus.de/politik/ausland/interview_aid_64500.html 
Földes, C. (2005). Kulturgeschichte, Kulturwissenschaft und Phraseologie: deutsch-ungarische Beziehungen. In I. Hausner, P. Wiesinger (unter Mitwirkung von K. Korecky-Kröll) (Hrsg.): Deutsche Wortforschung als Kulturgeschichte. Beiträge des Internationalen Symposiums aus Anlass des 90-jährigen Bestandes der Wörterbuchkanzlei der Österreichischen Akademie der Wissenschaften. Wien, 25.-27. September 2003. Wien: Verlag der Österreichischen Akademie der Wissenschaften, S. 323-345.

Furmanova, W. (1993). Interkulturelle Kommunikation und linguistische Landeskunde in Theorie und Praxis des Fremdsprachenunterrichts. Original: Фурманова, В.П. (1993). Межкультурная коммуникация и лингвокультуроведение в теории и практике обучения иностранным языкам. Саранск: Издат. Мордовского университета.

Furmanowskaja, N., Akischina, A. (1982). Russische Sprachetikette im sprachlandeskundlichen Aspekt. Original: Формановская, Н.Л., Акишина, А.Л. (1982). Русский речевой этикет в лингвострановедческом аспекте. In Верещагин Е.M. (Hrsg.), Словари и лингвострановедение. Москва: Русский язык.

Galskaja, N. (2004). Die Theorie des Fremdsprachenunterrichts. Original: Гальскова, Н.Д. (2004). Теория обучения иностранным языкам. Лингводидактика и методика. Москва: Айрис-Пресс.

Gehring, W. (2002). Kulturelle Kontexte in Sprachlehrgängen an Haupt- und Realschule. In Volkmann, Stierstorfer, Gehring (Hrsg.), a.a.O., S. 69-97.

Geistmann, Ch. (2002). Interkulturelle Kompetenz - eine wichtige und förderbare Fähigkeit in der internationalen Zusammenarbeit: Entwicklung eines Konzeptes zur schrittweisen Förderung interkultureller Kompetenz aufgezeigt am Beispiel der Handelspartner Frankreich und Deutschland. Norderstedt: Books on Demand.

Gick, A. (1997). Negotiating common Ground: Ein wesentliches Element (interkultureller) kommunikativer Kompetenz. In Bredella, Christ, Legutke (Hrsg.), a.a.O., S. 178-190.

Gröning, K. (2006). Pädagogische Beratung. Konzepte und Positionen. Wiesbaden: VS Verlag für Sozialwissenschaften.

Gruschevskaja T., Popkov, W., Sadochin A. (2002). Die Grundlagen der interkulturellen Kommunikation. Original: Грушевицкая, Т.Г., Попков, В.Д., Садохин, А.П. (2002). 
Основы межкультурной коммуникации: Учебник для вузов. Москва: ЮНИТИДАНА.

Haleeva, I. (1995). Die zweite Sprecherpersönlichkeit als Empfänger des fremden Textes. Original: Халеева, И.И. (1995). Вторичная языковая личность как реципиент инофонного текста. In Язык-система. Язык- текст. Язык - способность (S. 277-278). Москва: Издательство Российской академии наук.

Hall, E. T., Hall, M. R. (1989). Understanding Cultural Differences: keys to success in West Germany, France and the United States. Yarmouth: Intercultural Press.

Hennig Ch., Wahl D., Welker Ph. (2003). Mit Fremdheitserfahrungen umgehen lernen. Simulation. Verhandlung zwischen Honkas und Eugenikern. Letzter Zugriff am 26.06.2007 unter http://www.ikkompetenz.thueringen.de/downloads/simulation_honkas.pdf

Herbrand, F. (2000). Interkulturelle Kompetenz. Wettbewerbsvorteil in einer globalisierenden Wirtschaft. Grundlagen - Interkulturelles Training in Theorie und Praxis - Handlungsempfehlungen. Bern u.a.: Verlag Paul Haupt.

Hilpert, S., Kerner, M., Specht, F., Wagner, D., Weers, D., Reimann, M., Tomaszewski, A. (2009). Schritte 6. Ismaning: Max Hueber.

Hofner Saphiere, D. M.. Ecotonos: A Multicultural Problem-Solving Simulation. In Fowler, S. M./ Mumford M. (Hrg.). Intercultural Sourcebook: Cross-Cultural Training Methods Vol. 1, Yarmouth: Intercultural Press.

Hofstede, G. (1980). Culture's consequences: International differences in work-related values. Newbury Park, CA: Sage.

Hofstede, G. (1993). Interkulturelle Zusammenarbeit. Wiesbaden: Gabler.

Hölscher, P. (Hrsg.) (1997). Dil Dominosu - Sprachdomino zum Erlernen einfacher Redemittel in verschiedenen Sprachen. LIFE. Ideen und Materialien für interkulturelles Lernen. München: BMW-Group.

House, J. (1996). Zum Erwerb interkultureller Kompetenz im Unterricht des Deutschen als Fremdsprache. In „Zeitschrift für interkulturellen Fremdsprachenunterricht“ (Online), Nr. 1(3). Letzter Zugriff am 14.10.2010 unter http://zif.spz.tu-darmstadt.de/jg-01- 
Hymes, D. (1972). On Communicative Competence. In J. B. Pride, J. Holmes (Hrsg.), Sociolinguistics (S. 269-293). Harmondsworth: Penguin.

Irkutsker Forschungszentrum für organisierte Kriminalität. Original: Иркутский центр по изучению организованной преступности и коррупции (о.Ј.). Общая характеристика современной миграционной ситуации. Letzter Zugriff am 15.02.2006 unter http://irkcenter.isea.ru/rezult/nauch_isled/20.htm

Kallenbach, Ch. (1997). Fremdverstehen- aber wie? Ein Verfahren zur Anbahnung von Fremdverstehen. In Zeitschrift für interkulturellen Fremdverstehn (Online), 1(3). Letzter Zugriff am 13.10.2010 unter http://zif.spz.tu-darmstadt.de/jg-01-3/beitrag/kallenba.htm

Keller, G. (1991). Stereotypes in intercultural communication: effects of German-British pupil exchanges. In Buttjes, Byram (Hrsg.), a.a.O., S. 120-135.

Keller, G. (2007). Didaktik des interkulturellen Lernens aus neurobiologisch-konstruktivistischer und sozialpsychologischer Sicht. Letzter Zugriff am 14.10.2010 unter http://ourworld.compuserve.com/homepages/michaelwendt/Seiten/Keller.htm

Knapp-Potthoff, A. (1997). Interkulturelle Kommunikationsfähigkeit als Lernziel. In A. Knapp-Potthoff, M. Liedtke (Hrsg.), Aspekte interkultureller Kommunikationsfähigkeit. München: Iudicium, S. 181-205.

Knapp, K., Knapp-Potthoff, A. (1990): Interkulturelle Kommunikation. In „Zeitschrift für Fremdsprachenforschung“, Nr. 1/ 1990, S. 62-93.

Köck, P. (1995). Praxis der Unterrichtsgestaltung und des Schullebens. 2. Auflage. Donauwörth: Ludwig Auer.

Kravtschenko А. (2000). Kulturwissenschaft: Wörterbuch. Original: Кравченко, А.И. (2000). Культурология: Словарь. Москва: Академический проект.

Lacey, L., Trowbridge, J. (1995): Using the Case Study as a Training Tool. In S.M. Fowler, M.G. Mumford (Hrsg.), Intercultural Sourse Book: Cross-Cultural Trainings Methods. Yarmouth, Maine: Intercultural Press, S. 187-195. 
Lassera G. (1974). Realien der französischen Kultur im Französischunterrichts. Original: Ласера, Ж. (1974). Реалии французской культуры на уроках французского языка. In Верещагин, Е.И., Костомаров, В.Г. (Hrsg.), Лингвострановедческий аспект преподавания русского языка иностранцам (S. 218-219). Москва: Русский язык.

Leban, S., Premzl B., Puklavec E. (1997). Missverständnisse: Spagetti für zwei. In LIFE. Ideen und Materialien für interkulturelles Lernen. München: BMW-Group

Lencova, I.(1997). Andere Länder in unseren Köpfen. In LIFE. Ideen und Materialien für interkulturelles Lernen. München: BMW-Group.

Lukjantschenkowa, M. (1998). Integrierte Entwicklung der interkulturellen Kompetenz in einem Übersetzungskurs. Original: Лукьянченкова, М.С. (1998). Интегральное формирование межкультурной компетенции в курсе практического перевода. In Формирование социолингвистической компетенции: проблемы и перспективы. Материалы международной научно-практической конференции (S. 26-33). Барнаул: Барнаульский государственный педагогический университет.

Malzewa, D. (1991). Landeskunde durch phraselogische Redewendungen. Original: Мальцева, Д.Г. (1991). Страноведение через фразеологизмы. Москва: Высшая школа.

Marenkowa, I., Novikowa L. (2001). Berufsbezogene Sprache und Entwicklung der interkulturellen Kompetenz (am Beispiel der französischen Sprache in Tourismus und Hotelwirtschaft. Original: Маренкова, И.Н., Новикова Л.Ю. (2001). Язык профессионального общения и формирование межкультурной компетенции (на примере французского языка туристического бизнеса и гостиничного дела). Zugriff am 26.06.2008 unter http://www.sgu.ru/departments/french_and_spanish/docs/2.html

Martinelli Silvio (Hrsg.) (2000): Interkulturelles Lernen. T-Kit. Straßburg: Verlag des Europarats.

Maslowa, N., Slusarenko, S., Bisgeimer V., Gorbunowa N. (1998). Deutsch. Lehrbuch für die Hochschulen des MdI Russlands. Original: Маслова Н.П., Слюсаренко С.Н., Бизгермер М.Я., Горбунова Н.Н. (1998). Немецкий язык. Учебник для образовательных учреждений МВД России. Омск:ГУИПП Омский дом печати.

Mayer, C.-H., Boness, Ch. (2004). Interkulturelle Mediation und Konfliktbearbeitung: 
Bausteine deutsch-afrikanischer Wirklichkeiten. Münster u.a.: Waxmann

Mietzel, G. (2007). Pädagogische Psychologie des Lernens und Lehrens. 8., überarbeitete und erweiterte Auslage. Göttingen: Hogrefe.

Narognaja, W. (o.J.). Nonverbale Formen der Sprachetikette als Zeichen der kulturellen Besonderheiten von Sprachbewusstsein eines Volkes. Original: Нарожная, В.Д. (о.Ј.). Невербальные формы речевого этикета как сигналы национально культурной специфики языкового сознания этноса. Zugriff am 24.07.2008 unter http://www.rusnauka.com/2_KAND_2008/Philologia/26012.doc.htm.

Nevirko, L. (2003). Die Entwicklung der kommunikativen Kompetenz im Rahmen eines neuen Ausbildungsmodells für Englischlehrer. die Невирко, Л.И. (2003). Original: Формирование коммуникативной компетенции в рамках новой модели подготовки учителей английского языка. Материалы X конференции. "Педагогика развития: становление компетентности и результаты образования в различных подходах". Красноярск: Институт психологии и педагогики развития (Красноярск). Zugriff am 29.11.2007 unter : http://old.ippd.ru/bibl/pedagog_razvitie/p9_f002.doc

Ostendorf, A. (2005). Die pädagogische Begleitung betrieblicher Auslandspraktika - am Beispiel des Projektes FILIPA, Wirtschaftspädagogische Arbeitstexte, LMU München. Letzter Zugriff am 24.02.2007 unter www.wipaed.bwl.uni-muenchen.de

Pjantschina S., Mosejtschuk N., Paschkowa G. (2009) Deutsch für zukünftige Milizbeamte. Original: Пятчина C.B., Мосейчук Н. А., Пашкова Г. Н. (2009). Немецкий язык для будущтх сотрудников милиции. Иркутск: Восточно-Сибирский институт МВД.

Pjatschina S. (Hrsg.) (2002). Deutsch für zukünftige Brandschutzingenieure. Original: Пятчина С.В. (Под ред.) (2002). Немецкий язык: учебник для образовательных учреждений МВД России. Иркутск: Восточно-Сибирский инстиут МВД России.

Pschenkina, T. (1998). Die national-kulturellen Besonderheiten phraseologischer Redewendungen. Original: Пшенкина, Т.Г. (1998). Национально-культурная специфика фразеологических единиц. In Формирование социально-культурной компетенции: проблемы и перспективы. Материалы международной научно-практической конференции (S. 60-69). Барнаул: Барнаульский государственно-педагогический 
университет.

Richter, R. (1998). Interkulturelles Lernen via Internet? In Zeitschrift für interkulturellen Fremdsprachenunterricht (online), 3 (2). Letzter Zugriff am 01.03.2010 unter http://zif.spz.tu-darmstadt.de/jg-03-2/beitrag/richter1.htm

Ridkina, I. (2000). Zum Problem: Entwicklung der Selbstreflexion. Original: Рыдкина, И.Н. (2000). К постановке проблемы: обучение навыкам рефлекторного мышления. In Язык и образование в диалоге культур. Материалы 44 научно-методической конференции преподавателей и студентов (S. 108-114). Ставрополь: Издательство Ставропольского ГУ.

Risager, K. (1997). Language teaching and the process of European integration. In M. Byram, M. Fleming (Hrsg.), Language Learning in intercultural Perspective. Cambridge: University Press, S. 242-244.

Roche, J. (2001). Interkulturelle Sprachdidaktik. Eine Einführung. Tübingen: Narr.

Rokeach, M. (1973). The Nature of Human Values. New York: Free Press.

Rösch, O. (1998). Mit Stereotypen leben? Wie Deutsche und Russen sich heute sehen. In Rösch, O. (Hrsg.) Interkulturelle Kommunikation in den Geschäftsbeziehungen zwischen Russen und Deutschen. Wildauer Schriftenreihe Interkulturelle Kommunikation, Bd. 1. Berlin: News \& Media, S. $51-64$.

Schaub H., Zenke K.G. (1997). Wörterbuch Pädagogik.München: dtv.

Schinschke, A (1995). Perspektivenübernahme als grundlegende Fähigkeit im Umgang mit Fremden. In L. Brandella, H. Christ (Hrsg.), Didaktik des Fremdverstehens. Tübingen: Günter Narr, S. 36-50.

Scholz, R. (2008). „Kulturelle Identität. Über Multikulturalität im Unterschied zu Multikulturalismus“. Politische Meinung 2008 (8) (Hrsg.: B. Vogel), Nr. 465. Sankt Augustin: A. Fromm.

Schroll-Mahl, S. (2003). Die Deutschen - Wir Deutsche. Fremdwahrnehmung und Selbstsicht im Berufsleben. Göttingen: Vandenhoeck \& Ruprecht. 
Sepir, E. (1993). Auslese: Sprach- und Kulturwissenschaft. Original: Сепир, Э. (1993). Избранные труды по языкознанию и культуралогии. Москва: Издательская группа «Прогресс».

Serebrjannikowa, S. (2000). Interkulturelle Germanistik: Probleme und Aussichten. Original: Серебрякова, С.В. (2000). Межкультурная германистика: проблемы и перспективы. In Язык и образование в диалоге культур. Материалы 44 научнометодической конференции преподавателей и студентов (S.101-107). Ставрополь: Издательство Ставропольского ГУ.

Shirts, R. (1977). Bafa Bafa - A cross culture simulation. Del Mar, CA: Simile Two.

Sorokin, J. (1982). Lakunen als Zeichen der Einzigartigkeit einer sprachkulturellen Gemeinschaft. Original: Сорокин, Ю.А. (1982). Лакуны как сигналы специфики лингвокультурной общности. Национально-культурная специфика речевого общения народов CCCP, S.22-27. Москва: Наука.

Stepanow, J. (1985). Im dreidimensionalen Raum einer Sprache: semiotische Probleme von Linguistik, Philosophie und Kunst. Original: Степанов, Ю.С. (1985). В трехмерном пространстве языка: Семиотические проблемы лингвистики, философии, искусства. Москва: Издательство московского университета.

Stepanowa M. (1979). Wörterbuch für wortbildende Partikel in der deutschen Sprache. Original: Степанова, М.Д. (Hrsg.) (1979). Словарь словообразовательных элементов немецкого языка. Москва: Русский язык.

Szablewski-Cavus, P. (o.J.). „Ich habe da ganz andere Erfahrungen...“ Perspektivenwechsel, Gesprächsstile und vorgefasste Meinungen im Unterricht Deutsh als Zweitsprache. Letzter Zugriff am 12.02.2011 unter http://www.dazdidaktik.de/Szablewski_InterkulturellesLernen.pdf

Testzentrale (2008): Testkatalog 2008/2009. Göttingen: Hogrefe.

Thomas, A. (Hrsg.) (1993). Kulturvergleichende Psychologie. Göttingen: Hogrefe.

Thompson, M., Ellis, R., Wildavsky, A. (1990): Cultural Theory. Boulder Colo.: Westview Press: Westport, Conn.: Praeger. 
Tolkiehn, B. (1997). Interkulturelle Kommunikation im Fremdsprachenunterricht. Eine kritische Betrachtung. In I. Breuer, A. Sölter (Hrsg.), Der fremde Blick. Perspektiven interkultureller Kommunikation und Hermeneutik. Ergebnisse der DAAD-Tagung in London, 17.19. Juni 1996. Bozen: edition sturzflüge, S. 295-300.

Tomachin (1988). Amerikanische Realen. Lehrbuch für Landeskunde. Original: Томахин Г.Д. (1988). Реалии-американизмы. Пособие по становедению. Москва: Высшая школа.

Trim, J. (2006). Gemeinsamer europäischer Referenzrahmen für Sprachen: Lernen, lehren und beurteilen. Berlin: Langenscheidt.

Van Ek (1975). The Treshold Level. Strasbourg: Council of Europa.

Volkmann, L. (1999). Kriterien und Normen bei der Evaluation der Lehrwerke. In Börner, Vogel (Hrsg.), a.a.O., S. 117-144.

Volkmann, L. (2002). Aspekte und Dimensionen interkultureller Kompetenz. In Volkmann, Stierstorfer, Gehring (Hrsg.), a.a.O., S. 11-47.

Vollmer H. J. (1995). Diskurslernen und interkulturelle Kommunikationsfähigkeit. Der Beitrag der Pragmatik und der Diskursanalyse zu einem erweiterten Sprachlernkonzept. In Brandella (Hrsg.), a.a.O., S. 104-127.

Vollmer, H. J. (1994). Interkulturelles Lernen - interkulturelles Kommunizieren: Vom Wissen zum sprachlichen Handeln. In Bausch, Christ, Krumm (Hrsg.), a.a.O., S. 172-185.

Vollmer, H. J. (1995). Fremdsprachenlernen in multikulturellen Gesellschaften. In Brandella (Hrsg.), a.a.O., S. 495-508.

Werestschagin, E., Kostomarov, W. (1983). Sprache und Kultur. Original: Верещагин, Е.М., Костомаров, В.Т. (1983). Язык и культура. Москва: Русский язык.

Yoosefi T., Thomas A. (2008). Beruflich in Russland. Trainingsprogramm für Manager, Fach- und Führungskräfte. Göttingen: Vandenhoeck \& Ruprecht.

Zernizkaja A. (2005). Die Struktur des Begriffs „Kommunikative Kompetenz“. Original: Зерницкая, А.А. (2005). Структура понятия «Коммуникативная компетенция». 
Русский язык за рубежом, 2005 (1-2).

Zeuner, U. (1997). Landeskunde und interkulturelles Lernen in den verschiedenen Ausbildungsbereichen „Deutsch als Fremdsprache“ in der technischen Universität Dresden. Zeitschrift für interkulturellen Fremdspracheunterricht [Online], 2(1). Zugriff am 2.10.2007 unter http://www.spz.tu-darmstadt.de/projekt_ejournal/jg_02_1/ beitrag/ zeuner.htm Zubtschenko, E., Pankow, A. (2006). Unsere Miliz schützt sie. Original: Зубченко, E., Панков, А.(2006). Наша милиция их бережет. In Новые известия, 28.09.2006. Letzter Zugriff am 29.09.2006 unter http://www.rambler.ru/db/news/print.html?mid=8790837 
Anhang 1

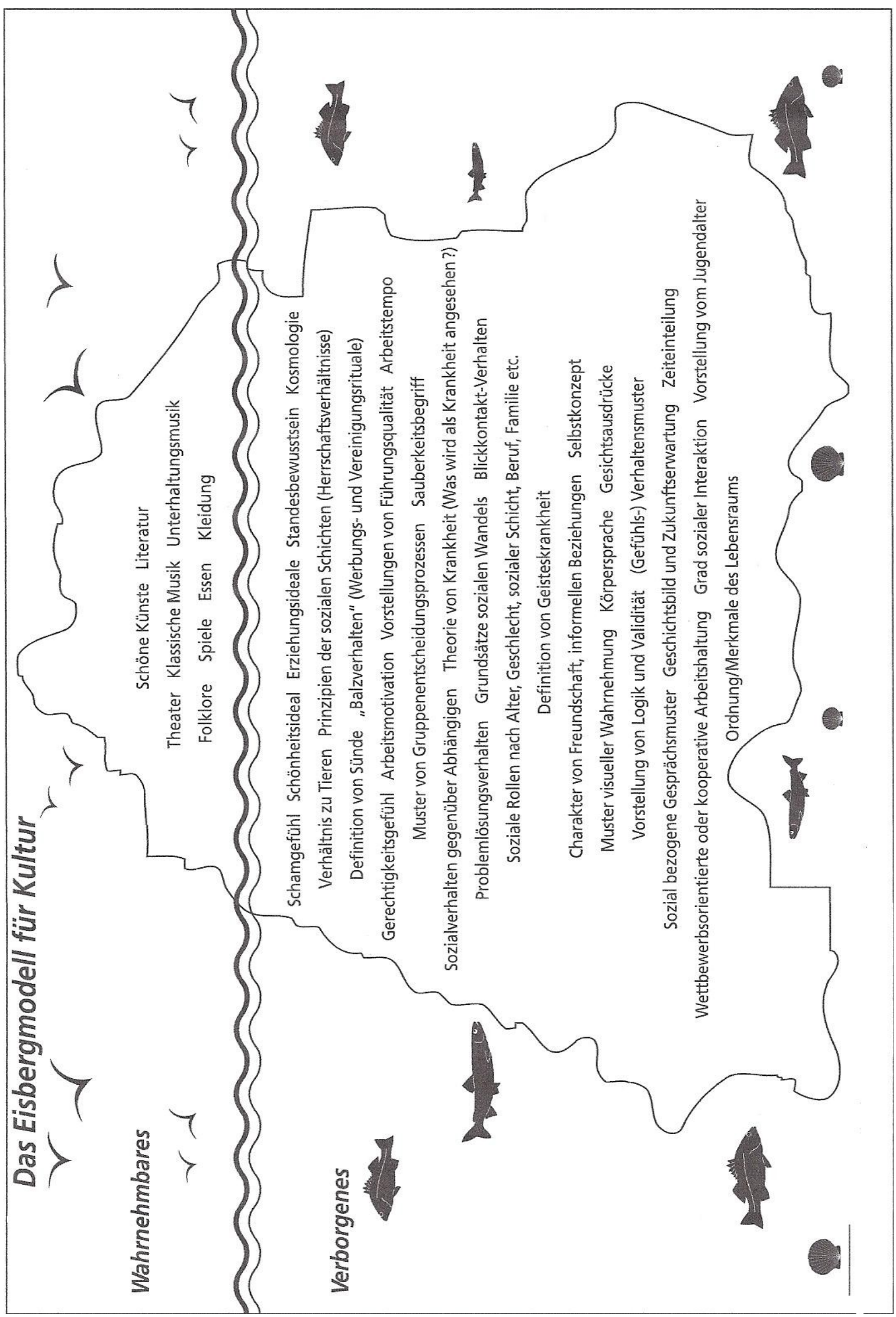

Quelle: Martinelli 2000, S.19 
Anhang 2

Deutschland, Deutschland über alles, Über alles in der Welt, Wenn es stets zu Schutz und Trutze Brüderlich zusammenhält, Von der Maas bis an die Memel, Von der Etsch bis an den Belt Deutschland, Deutschland über alles, Über alles in der Welt!

Deutsche Frauen, deutsche Treue, Deutscher Wein und deutscher Sang Sollen in der Welt behalten Ihren alten schönen Klang, Uns zu edler Tat begeistern Unser ganzes Leben lang Deutsche Frauen, deutsche Treue, Deutscher Wein und deutscher Sang!

Einigkeit und Recht und Freiheit Für das deutsche Vaterland!

Danach lasst uns alle streben Brüderlich mit Herz und Hand! Einigkeit und Recht und Freiheit Sind des Glückes Unterpfand Blüh im Glanze dieses Glückes, Blühe, deutsches Vaterland!
Ты миру отдана на травлю, И счета нет твоим врагам, Ну, как же я тебя оставлю? Ну, как же я тебя предам?

И где возьму благоразумье: "За око-око, кровь-за кровь", Германия-мое безумье! Германия-моя любовь!

Ну, как же я тебя отвергну, Мой столь гонимый Vaterland? Где все еще по Кенигсбергу Проходит узколицый Кант,

Где Фауста нового лелея В другом забытом городкеGeheimrath Goethe? по аллее Проходит с тросточкой в руке.

Ну, как же я тебя покину, Моя германская звезда, Когда любить наполовину Я не научена, -- когда, --

-- От песенок твоих в восторге -Не слышу лейтенантских шпор, Когда мне свят святой Георгий Во Фрейбурге, на Schwabenthor?.

Когда меня не душит злоба На Кайзера взлетевший ус, Когда в влюбленности до гроба Тебе, Германия, клянусь.

Нет ни волшебней, ни премудрей Тебя, благоуханный край, Где чешет золотые кудри Над вечным Рейном-Лорелей. 